\title{
Sequential Energy Transfer Catalysis: A Cascade Synthesis of Angularly-Fused Dihydrocoumarins
}

\author{
Tomáš Neveselý, Constantin G. Daniliuc, Ryan Gilmour \\ Organisch-Chemisches Institut, Westfälische Wilhelms-Universität Münster, Corrensstraße 40, \\ 48149 Münster, Germany
}

Corresponding Author: ryan.gilmour@uni-muenster.de

- General Information $\quad$ S2

- Experimental Section $\quad$ S4

- Crystallographic data $\quad$ S32

- References $\quad$ S34

- NOESY of key signals in compounds $\mathbf{2 , 1 3}, \mathbf{1 3 - Z} \quad$ S35

- NMR Spectra of Key Compounds S38 


\section{General Information}

All chemicals were purchased as reagent grade and used without further purification. Solvents for purification (extraction and chromatography) were purchased as technical grade and distilled on the rotary evaporator prior to use. For column chromatography $\mathrm{SiO}_{2}(40-63 \mu \mathrm{m}$ for Flash-Chromatography, VWR Chemicals) was used as stationary phase. Analytical thin layer chromatography (SiO2, TLC) was performed on aluminum foil pre-coated with $\mathrm{SiO}_{2}-60 \mathrm{~F}_{254}$ (Merck) and visualized with a UV-lamp (254 nm) or permanganate staining solution. NMR spectra were measured by the NMR service of the Organisch-Chemisches Institut, Westfälische Wilhelms-Universität Münster on a Bruker AV300, Bruker AV400, Agilent DD2 500 or an Agilent DD2 600 spectrometer at room temperature. The chemical shifts are referenced to the residual solvent peak as internal standard $\left(\mathrm{CDCl}_{3} \delta_{\mathrm{H}}=7.26 \mathrm{ppm}, \delta_{\mathrm{C}}=77.16 \mathrm{ppm} ; \mathrm{CD}_{3} \mathrm{CN} \delta_{\mathrm{H}}=\right.$ $1.94 \mathrm{ppm}$ ). The resonance multiplicity is abbreviated as: s (singlet), $\mathrm{d}$ (doublet), $\mathrm{t}$ (triplet), q (quadruplet), p (pentet), sext (sextet), sep (septet), m (multiplet) and b (broad). All NMR shifts are given in ppm. Assignments of unknown compounds are based on DEPT, COSY, HMBC, HSQC and NOESY spectra. Melting points were measured on a Büchi B-545 melting-point apparatus in open capillaries. IR spectra were recorded on a Perkin-Elmer 100 FT-IR spectrometer, selected adsorption bands are reported in wavenumbers $\left(\mathrm{cm}^{-1}\right)$ and intensities are reported as: w (weak), m (medium), s (strong) and b (broad). High-resolution mass spectra (HRESI) were measured by the MS service of the Insitute for Organic Chemsitry, Westfälische Wilhelms-Universität Münster. Photochemical reactions were performed utilizing a set-up of 4 Winger WEPUV3-S2 UV Power LED Star (Schwarzlicht) 1.2 W lamps (emission spectrum see Figure 1). The forward current per chip was set to $700 \mathrm{~mA}$, the resulting forward voltage was $3.4 \mathrm{~V}$ while the resulting radiant flux was $1200 \mathrm{~mW}$. The distance between the reaction vessels and the LEDs was set at approximately $0.5 \mathrm{~cm}$ for all reactions. While performing photochemical reactions, temperature of the setup (Figure 1) equilibrated at $50-51^{\circ} \mathrm{C}$. 

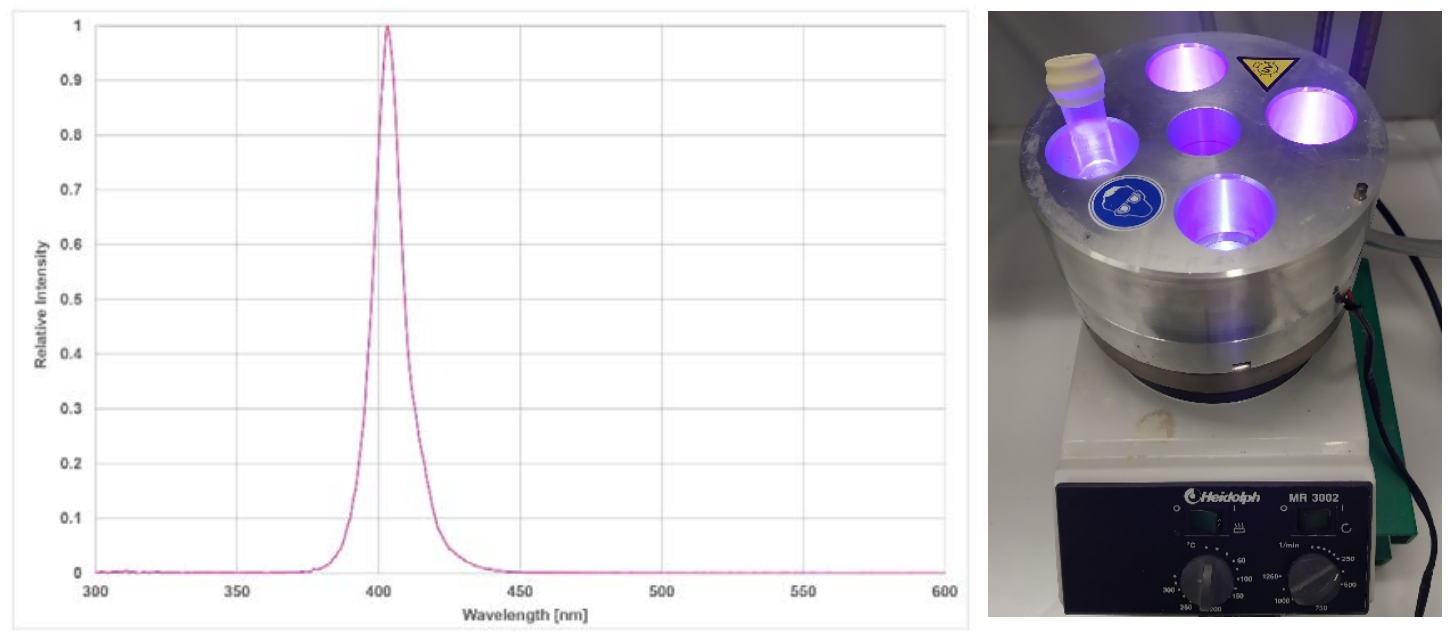

Figure 1 Emission spectrum of Winger WEPUV3-S2 UV Power LED Star (Schwarzlicht) 1.2 W (left). Custom build photoreactor used in this work (right). 


\section{Experimental Section}

\section{Preparation of Starting Materials}

\section{2-(Methoxymethoxy)benzaldehyde (S-1)}

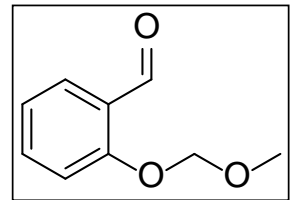

To a mixture of sailicylaldehyde (1.22g, $10 \mathrm{mmol}, 1.0$ eq.) and potassium carbonate (2.76g, $20 \mathrm{mmol}, 2.0$ eq.) in acetonitrile (20 mL) methoxymethyl chloride (0.97g, $12 \mathrm{mmol}, 1.2$ eq.) was added and the reaction mixture was stirred at RT for 1 hour. After completion $\mathrm{CH}_{2} \mathrm{Cl}_{2}(40 \mathrm{~mL})$ was added and organic phase was washed with water $(50 \mathrm{~mL})$, a solution of sodium hydroxide $(0.5 \mathrm{M}, 20 \mathrm{~mL})$, dried over $\mathrm{MgSO}_{4}$ and concentrated in vacuo. After purification of the crude product with column chromatography $\left(\mathrm{SiO}_{2}, n\right.$-pentane:diethyl ether = 95:5), a colorless oil was obtained (1.59 g, 96\%).

$\mathbf{R}_{\boldsymbol{f}}=0.20$ (n-pentane:diethyl ether = 9:1); ${ }^{1} \mathbf{H}$ NMR (300 MHz, Chloroform- $d$ ) $\delta 10.51$ (d, $J=0.8 \mathrm{~Hz}, 1 \mathrm{H}$ ), 7.85 (dd, $J=7.7,1.9 \mathrm{~Hz}, 1 \mathrm{H}$ ), 7.53 (ddd, $J=8.4,7.3,1.9 \mathrm{~Hz}, 1 \mathrm{H}$ ), 7.22 (dd, $J=8.4,1.0 \mathrm{~Hz}, 1 \mathrm{H}), 7.14-7.03$ (m, 1H), 5.31 (s, 2H), 3.53 (s, 3H); ${ }^{13} \mathrm{C}$ NMR (75 MHz, Chloroform- $d$ ) $\delta 189.8,159.7,135.9,128.4,125.5,121.9,115.0,94.6,56.5$.

Data in agreement with literature. ${ }^{[1]}$

\section{1-(2-Methoxymethoxyphenyl)hex-5-en-1-ol (S-2)}

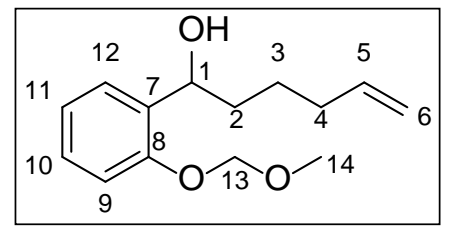

An oven dried Schlenk tube under and argon atmosphere was charged with magnesium (0.53 g, 22 mmol, $2.2 \mathrm{eq})$, catalytic iodine and was heated with a heat gun until vapors of iodine appeared. After 5 minutes dry THF (20 mL) was added followed by 5-bromopent-1-ene (1.64 g, 11 mmol, 1.1 eq.). The mixture was stirred at RT for 1 hour followed by addition of a solution of aldehyde S-1 (1.66 g, 10 mmol, 1 eq.) in THF (10 mL). The reaction mixture was stirred for an additional 30 minutes and then quenched with saturated ammonium chloride solution $(5 \mathrm{~mL})$. The reaction mixture was diluted with $\mathrm{CH}_{2} \mathrm{Cl}_{2}(20 \mathrm{ml})$ and washed with water $(50 \mathrm{~mL})$. The organic layer was dried with $\mathrm{MgSO}_{4}$ and concentrated in vacuo. After purification of the crude product with column chromatography $\left(\mathrm{SiO}_{2}, n\right.$-pentane:diethyl ether $\left.=7: 3\right)$, a colorless oil was obtained (2.07 g, 88\%).

$$
\mathbf{R}_{\boldsymbol{f}}=0.25 \text { (n-pentane:diethyl ether = 7:3); }{ }^{1} \mathbf{H} \text { NMR (300 MHz, Chloroform- } d \text { ) } \delta 7.35
$$
(dd, $J=7.5,1.8 \mathrm{~Hz}, 1 \mathrm{H}$; H12), 7.22 (ddd, $J=8.3,7.3,1.8 \mathrm{~Hz}, 1 \mathrm{H} ; \mathrm{H} 10$ ), 7.09 (dd, $J=8.3,1.2$ 
Hz, 1H; H9), 7.01 (td, $J=7.4,1.2 \mathrm{~Hz}, 1 \mathrm{H} ; \mathrm{H} 11$ ), 5.80 (ddt, $J=17.0,10.2,6.7 \mathrm{~Hz}, 1 \mathrm{H}$; H5), 5.23 (s, 2H; H13), 5.07 - 4.86 (m, 3H; H1, H6), 3.49 (s, 3H; H14), 2.35 (bs, 1H; OH), 2.20 1.99 (m, 2H; H4), 1.90 - 1.72 (m, 2H; H2), 1.63 - 1.39 (m, 2H; H3); ${ }^{13}$ C NMR (75 MHz, Chloroform-d) $\delta 154.3$ (C8), 138.9 (C5), 133.4 (C7), 128.4 (C10), 127.0 (C11), 122.1 (C12), 114.7 (C6), 114.1 (C9), 94.5 (C13), 70.5 (C1), 56.4 (C14), 37.0 (C3), 33.7 (C2), 25.4 (C4); HRESI-MS: m/z $259.1302\left([\mathrm{M}+\mathrm{Na}]^{+}\right.$, calcd. for $\mathrm{C}_{14} \mathrm{H}_{20} \mathrm{NaO}_{3}{ }^{+}:$259.1305); IR (ATR): $\tilde{v}=3256$ (br), 2991 (w), 2920 (w), 2874 (w), 1532 (w), 1483 (w), 1410 (w), 1238 (w), 1201 (m), 1096 (w), $987(\mathrm{~m}), 880(\mathrm{w}), 746(\mathrm{w}) \mathrm{cm}^{-1}$.

\section{1-(2-(Methoxymethoxy)phenyl)hex-5-en-1-one (S-3)}

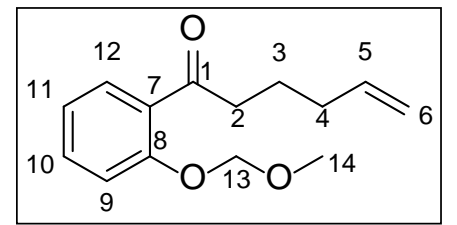

To a solution of alcohol S-2 (1.10 g, 4.65 mmol, 1 eq.) in $\mathrm{CH}_{2} \mathrm{Cl}_{2}$ (30 ml), pyridinium chlorochromate (1.30 g, $6.05 \mathrm{mmol}, 1.3$ eq.) was added. The reaction mixture was stirred for 1 hour at RT before dilution with $n$-pentane $(60 \mathrm{ml})$. Solids were removed by filtration and the filtrate was concentrated in vacuo. After purification of the crude product with column chromatography ( $\mathrm{SiO}_{2}, n$-pentane:diethyl ether =9:1), a colorless oil was obtained (0.55 g, $55 \%)$.

$\mathbf{R}_{\boldsymbol{f}}=0.25$ (n-pentane:diethyl ether = 9:1); ${ }^{1} \mathbf{H}$ NMR (400 MHz, Chloroform-d) $\delta 7.62$ (dd, $J=7.7,1.8 \mathrm{~Hz}, 1 \mathrm{H}$; H12), 7.41 (ddd, $J=8.4,7.3,1.8 \mathrm{~Hz}, 1 \mathrm{H} ; \mathrm{H} 10$ ), 7.17 (dd, $J=8.4,1.1$ Hz, 1H; H9), 7.04 (td, $J=7.4,1.0 \mathrm{~Hz}, 1 \mathrm{H} ; \mathrm{H} 11$ ), 5.81 (ddt, $J=16.9,10.2,6.7 \mathrm{~Hz}, 1 \mathrm{H}$; H5), 5.26 (s, 2H; H13), 5.06 - 4.94 (m, 2H; H6), 3.50 (s, 3H; H14), 3.03 - 2.95 (m, 2H; H2), 2.18 2.07 (m, 2H; H4), 1.88 - 1.75 (m, 2H; H3); ${ }^{13}$ C NMR (101 MHz, Chloroform-d) $\delta 203.2$ (C1), 155.9 (C8), 138.4 (C5), 133.1 (C10), 123.0 (C12), 129.8 (C7), 121.9 (C11), 115.2 (C9), 114.9 (C6), 94.6 (C13), 56.6 (C14), 43.1 (C2), 33.4 (C3), 23.6 (C4); HR-ESI-MS: m/z 257.1150 ([M+Na] $]^{+}$, calcd. for $\mathrm{C}_{14} \mathrm{H}_{18} \mathrm{NaO}_{3}{ }^{+}:$257.1154); IR (ATR): $\tilde{v}=2990(\mathrm{w}), 2863(\mathrm{w}), 1648(\mathrm{~s})$, 1588 (w), 1534 (w), 1490 (w), 1338 (w), 1214 (m), 1076 (w), 963 (m), 810 (w), 745 (w) cm².

\section{Ethyl (E)-3-(2-(methoxymethoxy)phenyl)octa-2,7-dienoate (13)}

Sodium hydride (160 mg, 4 mmol, 2 eq.; 60 \% in paraffin oil) was weighed into an oven dried Schlenk tube under argon atmosphere. The flask was sealed before the addition of THF (6 mL) 


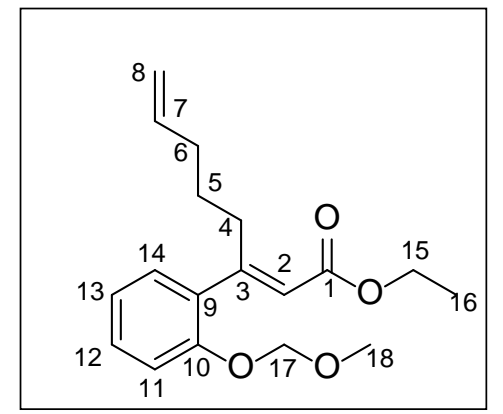

and dropwise addition of triethyl phosphonoacetate (0.90 g, 4 mmol, 2 eq.). Once the evolution of hydrogen stopped, a solution of ketone S-3 (0.46 g, 2 mmol, 1 eq.) in THF (2 mL) was added. The reaction mixture was stirred at RT for 16 hours and was quenched with a saturated ammonium chloride solution (5 mL). The reaction mixture was diluted with $\mathrm{CH}_{2} \mathrm{Cl}_{2}(20 \mathrm{~mL})$, washed with water $(20 \mathrm{~mL})$. Organics were separated and concentrated in vacuo. After purification of the crude product with column chromatography $\left(\mathrm{SiO}_{2}, n\right.$-pentane:diethyl ether $\left.=9: 1\right)$, a colorless oil was obtained (0.33 g, 54\%). Together with the desired product minor amount of $Z$ isomer 13-Z was obtained as a colorless oil (0.08g, 13\%).

13* - $\mathbf{R}_{\boldsymbol{f}}=0.30$ ( $n$-pentane:diethyl ether $\left.=9: 1\right) ;{ }^{1} \mathbf{H}$ NMR $(600 \mathrm{MHz}$, Chloroform- $d) \delta$ 7.30 - 7.24 (m, 1H; H12), 7.15 - 7.08 (m, 2H; H11, H14), 6.98 (td, $J$ = 7.4, 1.1 Hz, 1H; H13), 5.82 (t, $J=0.7 \mathrm{~Hz}, 1 \mathrm{H} ; \mathrm{H} 2$ ), 5.75 (ddt, $J=16.9$, 10.2, 6.7 Hz, 1H; H7), 5.17 (s, 2H; H18), 4.97 - 4.86 (m, 2H; H8), 4.20 (q, J = 7.1 Hz, 2H; H15), 3.46 (s, 3H; H18), 3.10 - 3.04 (m, 2H; H4), 2.09 - 2.02 (m, 2H; H6), $1.49-1.41$ (m, 2H; H5), 1.30 (t, $J=7.1 \mathrm{~Hz}, 3 \mathrm{H} ; \mathrm{H} 16) ;{ }^{13} \mathbf{C}$ NMR (151 MHz, Chloroform-d) $\delta 166.6$ (C1), 160.8 (C3), 154.1 (C10), 138.8 (C7), 132.6 (C9), 129.71 (C12), 129.47 (C14), 121.8 (C13), 119.7 (C2), 114.69 (C11), 114.65 (C8), 94.7 (C17), 59.9 (C15), 56.3 (C18), 34.0 (C5), 31.9 (C4), 27.8 (C6), 14.5 (C16); HR-ESI-MS: m/z $327.1576\left([\mathrm{M}+\mathrm{Na}]^{+}\right.$, calcd. for $\left.\mathrm{C}_{18} \mathrm{H}_{24} \mathrm{NaO}_{4}{ }^{+}: 327.1572\right)$; IR (ATR): $\tilde{v}=2961(\mathrm{w}), 2951(\mathrm{w})$, 2835 (w), 1710 (s), 1620 (m), 1456 (m), 1348 (m), 1321 (w), 1302 (w), 1223 (w), 1157 (s), 1123 (m), 1085 (m), 1014 (w), 916 (w), 875 (m), 768 (s) cm- ${ }^{1}$.

13-Z* - $\mathbf{R}_{\boldsymbol{f}}=0.24$ (n-pentane:diethyl ether = 9:1); ${ }^{1} \mathbf{H}$ NMR $(600 \mathrm{MHz}$, Chloroform- $d) \delta$ 7.27 - 7.21 (m, 1H), 7.11 (dt, J = 8.2, 0.7 Hz, 1H), 7.02 - 6.95 (m, 2H), 5.94 (t, J = 1.3 Hz, 1H), 5.76 (ddt, J = 16.9, 10.2, $6.6 \mathrm{~Hz}, 1 \mathrm{H}), 5.13$ (s, 2H), $5.02-4.92$ (m, 2H), 3.95 (q, J = $7.1 \mathrm{~Hz}$, 2H), 3.43 (s, 3H), $2.49-2.43$ (m, 2H), $2.12-2.05$ (m, 2H), $1.57-1.48$ (m, 2H), 1.03 (t, J = 7.1 Hz, 3H). ${ }^{13}$ C NMR (151 MHz, Chloroform-d) $\delta 165.9,156.9,153.5,138.3,130.5,128.8$, 128.6, 121.5, 118.6, 115.1, 114.5, 94.7, 59.7, 56.1, 39.0, 33.3, 26.6, 14.1.

*NOESY spectra of key contacts of both isomers are shown on pages 36 and 37. 
Ethyl (E)-3-(2-hydroxyphenyl)octa-2,7-dienoate (1)

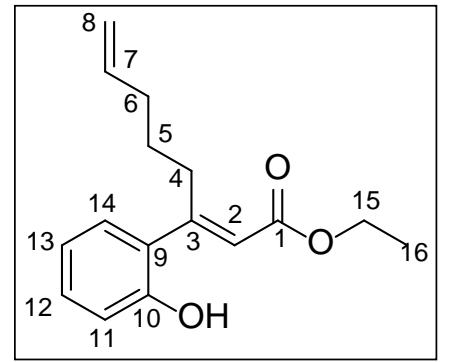

To a solution of 13 (243 mg, $0.8 \mathrm{mmol}$, 1eq.) in dry $\mathrm{CH}_{2} \mathrm{Cl}_{2}$ (5 mL), trimethylsilyl bromide (135 mg, $0.88 \mathrm{mmol}, 1.1 \mathrm{eq.)}$ was added. The mixture was stirred at RT for 2 hours and then ethanol $(2 \mathrm{~mL})$ was added. The reaction mixture was concentrated in vacuo. After purification of the crude product with column chromatography $\left(\mathrm{SiO}_{2}, n\right.$-pentane:diethyl ether =9:1), a colorless oil was obtained (155 mg g, 74\%).

$\mathbf{R}_{\boldsymbol{f}}=0.15$ ( $n$-pentane:diethyl ether $=9: 1$ ); ${ }^{1} \mathbf{H}$ NMR (300 MHz, Chloroform- $d$ ) $\delta 7.21$ (ddd, $J=$ 8.2, 7.4, 1.7 Hz, 1H; H12), 7.10 (dd, $J$ = 7.6, 1.7 Hz, 1H; H14), 6.96 - 6.85 (m, 2H; H11; H13), 5.92 (s, 1H; H2), 5.75 (ddt, $J=16.9,10.2,6.6$ Hz, 1H; H7), 5.20 (b, 1H; OH), 5.02 - 4.84 (m, 2H; H8), 4.21 (q, $J=7.1 \mathrm{~Hz}, 2 \mathrm{H} ; \mathrm{H} 15), 3.14$ - 2.96 (m, 2H; H4), 2.08 (q, $J=7.2 \mathrm{~Hz}, 2 \mathrm{H} ; \mathrm{H6}$ ), 1.56 - 1.41 (m, 2H; H5), 1.31 (t, $J=7.1 \mathrm{~Hz}, 3 \mathrm{H} ; \mathrm{H} 16) ;{ }^{13} \mathrm{C}$ NMR (75 MHz, Chloroform- $d$ ) $\delta$ 166.0 (C1), 158.4 (C3), 152.0 (C10), 138.3 (C7), 129.6 (C14), 128.6 (C9), 128.5 (C12), 120.6 (C13), 120.2 (C2), 116.0 (C11), 114.9 (C6), 60.2 (C15), 33.8 (C5), 32.5 (C4), 27.7 (C6), 14.3 (C16); HR-ESI-MS: m/z $283.1317\left([\mathrm{M}+\mathrm{Na}]^{+}\right.$, calcd. for $\mathrm{C}_{16} \mathrm{H}_{20} \mathrm{NaO}_{3}{ }^{+}:$283.1310); IR (ATR): $\tilde{v}=3250$ (br), 2871 (w), 1732 (s) 1510 (w), 1466 (m), 1422 (w), 1211 (m), 1123 (m), 1090 (w), 935 (m), $840(\mathrm{w}), 738(\mathrm{w}) \mathrm{cm}^{-1}$.

\section{5-Bromo-2-hydroxybenzaldehyde (S-4)}

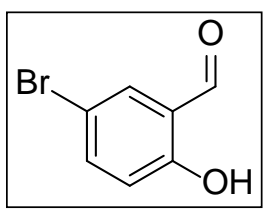

To a solution of salicylaldehyde (1.83 g, 15 mmol, 1 eq.) in chloroform (20 $\mathrm{mL}$ ), bromine (2.56 g, $16 \mathrm{mmol}, 1.07 \mathrm{eq}$ ) was added dropwise. The mixture was stirred at $45^{\circ} \mathrm{C}$ for 3 hours, and then, residual bromine was quenched with saturated aqueous sodium sulfite $(5 \mathrm{~mL})$. The organic phase was washed with water $(25 \mathrm{~mL})$, dried over $\mathrm{MgSO}_{4}$ and concentrated in vacuo. The crude product was recrystallized from isopropyl alcohol and product was obtained as a white crystalline powder (232 g, $77 \%)$.

${ }^{1}$ H NMR (300 MHz, Chloroform-d) $\delta 10.94(\mathrm{~d}, J=0.5 \mathrm{~Hz}, 1 \mathrm{H}), 9.84$ (d, $\left.J=0.7 \mathrm{~Hz}, 1 \mathrm{H}\right)$, $7.68(\mathrm{~d}, J=2.5 \mathrm{~Hz}, 1 \mathrm{H}), 7.60$ (ddd, $J=8.8,2.5,0.5 \mathrm{~Hz}, 1 \mathrm{H}), 6.91(\mathrm{dt}, J=8.9,0.5 \mathrm{~Hz}, 1 \mathrm{H})$; ${ }^{13}$ C NMR (75 MHz, Chloroform- $d$ ) $\delta$ 195.6, 160.7, 139.9, 135.8, 121.9, 112.0, 111.5.

Data in agreement with literature. ${ }^{[2]}$ 


\section{5-Bromo-2-(methoxymethoxy)benzaldehyde (S-5)}

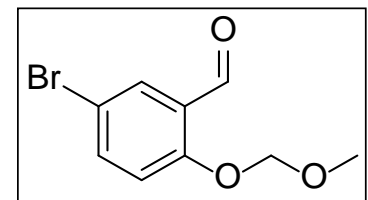

To a mixture of aldehyde S-4 (2.01 g, $10 \mathrm{mmol}, 1.0$ eq.) and potassium carbonate (2.76g, $20 \mathrm{mmol}, 2.0$ eq.) in acetonitrile $(20 \mathrm{~mL})$ methoxymethyl chloride $(0.97 \mathrm{~g}, 12 \mathrm{mmol}, 1.2$ eq.) was added. The reaction mixture was stirred at $\mathrm{RT}$ until the starting material was consumed (visible by TLC). After completion, the reaction mixture was diluted with $\mathrm{CH}_{2} \mathrm{Cl}_{2}(100 \mathrm{~mL})$, washed with water (50 mL) and washed with a solution of sodium hydroxide $(0.5 \mathrm{M}, 20 \mathrm{~mL})$. Organics were separated and dried over $\mathrm{MgSO}_{4}$ and concentrated in vacuo. The material was used without further purification (2.38 g, 97\%).

$\mathbf{R}_{\boldsymbol{f}}=0.40$ (n-pentane:diethyl ether = 7:3); ${ }^{1} \mathbf{H}$ NMR (400 MHz, Chloroform- $d$ ) $\delta 10.41$ (s, 1H), 7.93 (d, $J=2.6 \mathrm{~Hz}, 1 \mathrm{H}$ ), 7.60 (dd, $J=8.9,2.6 \mathrm{~Hz}, 1 \mathrm{H}$ ), 7.14 (d, $J=8.9 \mathrm{~Hz}, 1 \mathrm{H}$ ), 5.28 (s, 2H), 3.51 (s, 3H); ${ }^{13}$ C NMR (101 MHz, Chloroform-d) $\delta$ 188.4, 158.7, 138.4, 131.0, 126.8, 117.3, 114.9, 94.9, 56.7.

Data in agreement with literature. ${ }^{[3]}$

\section{1-(5-Bromo-2-(methoxymethoxy)phenyl)hex-5-en-1-ol (S-6)}

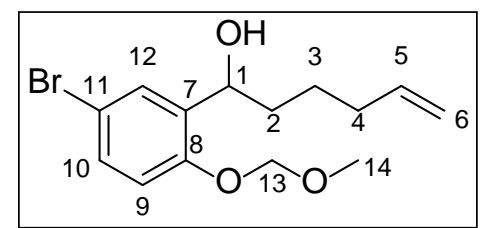

An oven dried Schlenk tube under an argon atmosphere was charged with magnesium ( $0.39 \mathrm{~g}, 16 \mathrm{mmol}, 2 \mathrm{eq}$.), catalytic iodine and heated with a heat gun until vapors of iodine appeared. After 5 minutes dry THF (30 mL) was added followed by 5-bromopent1-ene (1.34 g, 9 mmol, 1.13 eq.). The reaction mixture was stirred at RT for 1 hour followed by addition of a solution of aldehyde S-5 (1.96 g, $8 \mathrm{mmol}, 1$ eq.) in THF (10 mL). The reaction mixture was stirred for an additional 30 minutes and was then quenched with saturated ammonium chloride solution (5 mL) and diluted with $\mathrm{CH}_{2} \mathrm{Cl}_{2}(30 \mathrm{ml})$. The organic layer was washed with water $(50 \mathrm{~mL})$, dried over $\mathrm{MgSO}_{4}$ and concentrated in vacuo. After purification of the crude product with column chromatography $\left(\mathrm{SiO}_{2}, n\right.$-pentane:diethyl ether =7:3), a colorless oil was obtained (1.87 g, 74\%).

$\mathbf{R}_{\boldsymbol{f}}=0.43$ (n-pentane:diethyl ether = 7:3); ${ }^{1} \mathbf{H}$ NMR (300 MHz, Chloroform- $d$ ) $\delta 7.50$ (d, $J$ $=2.5 \mathrm{~Hz}, 1 \mathrm{H}$; H12), 7.30 (dd, $J=8.8,2.5 \mathrm{~Hz}, 1 \mathrm{H}$; H10), 6.97 (d, $J=8.7 \mathrm{~Hz}, 1 \mathrm{H} ; \mathrm{H9}$ ), 5.80 (ddt, $J=16.9,10.1,6.7 \mathrm{~Hz}, 1 \mathrm{H}$; H5), 5.19 (s, 2H; H13), 5.09 - 4.88 (m, 3H; H1, H6), 3.47 (s, 3H; H13), 2.22 (d, $J=5.3 \mathrm{~Hz}, 1 \mathrm{H} ; \mathrm{OH}), 2.15-2.03$ (m, 2H; H4), 1.76 (dt, $J=8.8,6.8 \mathrm{~Hz}, 2 \mathrm{H}$; H2), 1.66 - 1.37 (m, 2H; H3); ${ }^{13}$ C NMR (75 MHz, Chloroform-d) $\delta 153.2$ (C8), 138.70 (C5), 
135.80 (C7), 131.0 (C10), 129.9 (C12), 115.8 (C9), 114.9 (C6), 114.7 (C11), 94.6 (C13), 69.6 (C1), 56.4 (C14), 37.0 (C3), 33.7 (C2), 25.2 (C4); HR-ESI-MS: m/z 337.0419 ([M+Na]+, calcd. for $\mathrm{C}_{14} \mathrm{H}_{19} \mathrm{O}_{3} \mathrm{BrNa}^{+}$: 337.0415); IR (ATR): $\tilde{v}=3490$ (br), 2923 (w), 1680 (s), 1621 (m), 1401 (w), 1346 (w), 1340 (m), 1273 (m), 1169 (s), 1040 (m), 1007 (m), 922 (w), 875 (m), 821 (s), $746(\mathrm{w}), 728(\mathrm{w}) \mathrm{cm}^{-1}$

\section{1-(5-Bromo-2-(methoxymethoxy)phenyl)hex-5-en-1-one (S-7)}

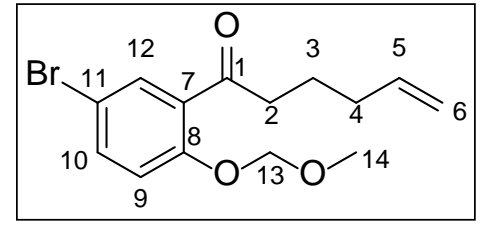

To a solution of alcohol S-6 (1.26 g, 4 mmol, 1 eq.) in $\mathrm{CH}_{2} \mathrm{Cl}_{2}$ (30 $\mathrm{ml})$, pyridinium chlorochromate (1.08 g, $5 \mathrm{mmol}, 1.25$ eq.) was added. The reaction mixture was stirred at RT for 1 hour before dilution with $n$-pentane $(60 \mathrm{~mL})$. Solids were removed by filtration and the filtrate was concentrated in vacuo. After purification of the crude product with column chromatography $\left(\mathrm{SiO}_{2}, n\right.$-pentane:diethyl ether $\left.=7: 3\right)$, a colorless oil was obtained (1.08 g, 86\%).

$\mathbf{R}_{\boldsymbol{f}}=0.70$ (n-pentane:diethyl ether = 7:3); ${ }^{1} \mathbf{H}$ NMR (400 MHz, Chloroform- $d$ ) $\delta 7.71$ (d, $J$ $=2.6 \mathrm{~Hz}, 1 \mathrm{H} ; \mathrm{H} 12$ ), 7.49 (dd, $J=8.8,2.6 \mathrm{~Hz}, 1 \mathrm{H} ; \mathrm{H} 10$ ), 7.08 (d, $J=8.8 \mathrm{~Hz}, 1 \mathrm{H} ; \mathrm{H9}$ ), 5.80 (ddt, $J=16.9,10.2,6.7 \mathrm{~Hz}, 1 \mathrm{H}$; H5), 5.23 (s, 2H; H13), 5.06 - 4.93 (m, 2H; H6), 3.49 (s, 3H; H14), 2.96 (t, $J=7.4$ Hz, 2H; H2), 2.16 - 2.08 (m, 2H; H4), $1.84-1.74$ (m, 2H; H3); ${ }^{13}$ C NMR (101 MHz, Chloroform-d) $\delta 201.4$ (C1), 154.8 (C8), 138.1 (C5), 135.5 (C7), 132.5 (C10), 131.1 (C12), 116.8 (C9), 115.2 (C6), 114.4 (C11), 94.6 (C13), 56.5 (C14), 42.9 (C2), 33.2 (C3), 23.3 (C4); HR-ESI-MS: m/z $335.0269\left([\mathrm{M}+\mathrm{Na}]^{+}\right.$, calcd. for $\mathrm{C}_{14} \mathrm{H}_{17} \mathrm{O}_{3} \mathrm{BrNa}^{+}$: 335.0253$)$; IR (ATR): $\tilde{v}=2980$ (w), 1676 (s), 1562(w), 1487 (m), 1366 (w), 1340 (m), 1273 (m), 1159(s), 1081 (m), 1042 (m), 1007 (m), 922 (w), 737 (w), 708 (w), cm

\section{Ethyl (E)-3-(5-bromo-2-(methoxymethoxy)phenyl)octa-2,7-dienoate (S-8)}

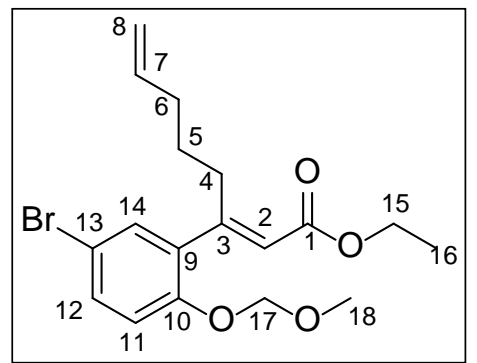

Sodium hydride (272 mg, $6.8 \mathrm{mmol}$, 2 eq.; 60\% in paraffin oil) was weighed into an oven dried Schlenk tube under argon atmosphere. The flask was sealed before the addition of THF (10 $\mathrm{mL}$ ) and dropwise addition of triethyl phosphonoacetate (1.52 g, $6.8 \mathrm{mmol}, 2$ eq.). Once the evolution of hydrogen stopped, a solution of ketone S-7 (1.06 g, 3.4 mmol, 1 eq.) in THF (4 mL) was added. The reaction mixture was stirred at RT for 16 hours and was quenched with saturated ammonium chloride solution 
(5 mL). The reaction was diluted with $\mathrm{CH}_{2} \mathrm{Cl}_{2}(25 \mathrm{~mL})$ and organics were washed with water (20 $\mathrm{mL})$ and concentrated in vacuo. After purification of the crude product with column chromatography $\left(\mathrm{SiO}_{2}, \quad n\right.$-pentane:diethyl ether $\left.=9: 1\right)$, a colorless oil was obtained (0.61 g, 47\%).

$\mathbf{R}_{\boldsymbol{f}}=0.75$ (n-pentane:diethyl ether = 7:3); ${ }^{\mathbf{1}} \mathbf{H}$ NMR (500 MHz, Chloroform- $d$ ) $\delta 7.36$ (dd, $J=8.8,2.5 \mathrm{~Hz}, 1 \mathrm{H}$; H12), 7.23 (d, $J=2.5 \mathrm{~Hz}, 1 \mathrm{H}$; H14), 7.01 (d, $J=8.8 \mathrm{~Hz}, 1 \mathrm{H} ; \mathrm{H} 11$ ), 5.80 - 5.79 (m, 1H; H2), 5.79 - 5.71 (m, 1H; H7), 5.14 (s, 2H; H17), 4.98 - 4.88 (m, 2H; H8), 4.20 (q, $J=7.1 \mathrm{~Hz}, 2 \mathrm{H} ; \mathrm{H} 15), 3.44$ (s, 3H; H18), 3.07 - 2.96 (m, 2H; H4), 2.10 - 2.01 (m, 2H; H6), 1.49 - 1.40 (m, 2H; H5), 1.30 (t, $J=7.1 \mathrm{~Hz}, 3 \mathrm{H} ; \mathrm{H16}) ;{ }^{13}$ C NMR (126 MHz, Chloroform- $d$ ) $\delta$ 166.2 (C1), 159.1 (C3), 153.3 (C10), 138.6 (C7), 134.5 (C9), 132.19 (C14), 132.09 (C12), 120.4 (C2), 116.4 (C11), 114.9 (C8), 114.2 (C13), 94.7 (C17), 60.1 (C15), 56.4 (C18), 33.9 (C5), 31.7 (C4), 27.8 (C6), 14.4 (C16); HR-ESI-MS: m/z 405.0685 ([M+Na $]^{+}$, calcd. for $\mathrm{C}_{18} \mathrm{H}_{23} \mathrm{O}_{4} \mathrm{BrNa}^{+}$: 405.0672); IR (ATR): $\tilde{v}=2945$ (w), 2847 (w), 1733 (s), 1655 (m), 1541 (m), 1511 (w), 1478 (w), $1221(\mathrm{~m}), 1195$ (w), $1150(\mathrm{w}), 1036$ (m), $930(\mathrm{w}), 827(\mathrm{~m}) \mathrm{cm}^{-1}$.

\section{Ethyl (E)-3-(5-bromo-2-hydroxyphenyl)octa-2,7-dienoate (S-9)}

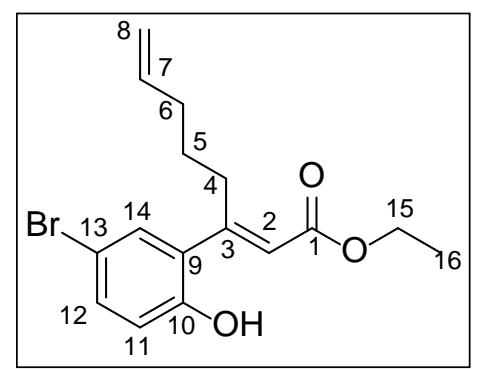

To a solution of compound S-8 (192 mg, $0.5 \mathrm{mmol}$, 1eq.) in dry $\mathrm{CH}_{2} \mathrm{Cl}_{2}$ (5 mL), trimethylsilyl bromide (107 mg, $0.7 \mathrm{mmol}, 1.4$ eq.) was added. The reaction mixture was stirred at RT for 2 hours before the addition of ethanol $(2 \mathrm{~mL})$ and the reaction mixture was concentrated in vacuo. After purification of the crude product with column chromatography $\left(\mathrm{SiO}_{2}, n\right.$-pentane:diethyl ether $\left.=9: 1\right)$, a

colorless oil was obtained (152 mg, 90\%).

$\mathbf{R}_{\boldsymbol{f}}=0.45$ (n-pentane:diethyl ether = 7:3); ${ }^{1} \mathbf{H}$ NMR (500 MHz, Chloroform- $d$ ) $\delta 7.30$ (ddd, $J=8.7,2.5,0.5 \mathrm{~Hz}, 1 \mathrm{H} ; \mathrm{H} 12$ ), 7.21 (d, $J=2.4 \mathrm{~Hz}, 1 \mathrm{H}$; H14), 6.78 (d, $J=8.6 \mathrm{~Hz}, 1 \mathrm{H}$; H11), 5.90 (t, $J=0.7 \mathrm{~Hz}, 1 \mathrm{H} ; \mathrm{H} 2$ ), 5.75 (ddt, $J=16.9,10.2,6.7 \mathrm{~Hz}, 1 \mathrm{H} ; \mathrm{H} 7$ ), 5.30 (s, 1H; OH), 5.02 - 4.87 (m, 2H; H8), 4.22 (q, $J=7.1$ Hz, 2H; H15), 3.06 - 2.94 (m, 2H; H4), 2.14 - 2.02 (m, 2H; H6), 1.48 (dtd, $J=10.3,7.7,5.5 \mathrm{~Hz}, 2 \mathrm{H} ; \mathrm{H} 5), 1.31$ (t, $J=7.2,3 \mathrm{H} ; \mathrm{H} 16) ;{ }^{13} \mathrm{C}$ NMR (126 MHz, Chloroform-d) $\delta 165.9$ (C1), 157.0 (C3), 151.5 (C10), 138.2 (C7), 132.4 (C12), 131.2 (C14), 130.8 (C9), 121.2 (C2), 118.0 (C11), 115.1 (C8), 112.8 (C13), 60.5 (C15), 33.9 (C5), 32.4 (C4), 27.8 (C6), 14.4 (C16); HR-ESI-MS: m/z 361.0411 ([M+Na $]^{+}$, calcd. for $\mathrm{C}_{16} \mathrm{H}_{19} \mathrm{O}_{3} \mathrm{BrNa}^{+}$: 361.0410$)$ ); IR (ATR): $\tilde{v}==3298$ (br), 2883 (w), 1740 (s), 1610 (m), 1511 (w), 1346 (w), $1221(\mathrm{~m}), 1195(\mathrm{w}), 1170(\mathrm{w}), 1042(\mathrm{~m}), 936(\mathrm{w}), 838(\mathrm{~m}) 778(\mathrm{w}) \mathrm{cm}^{-1}$. 


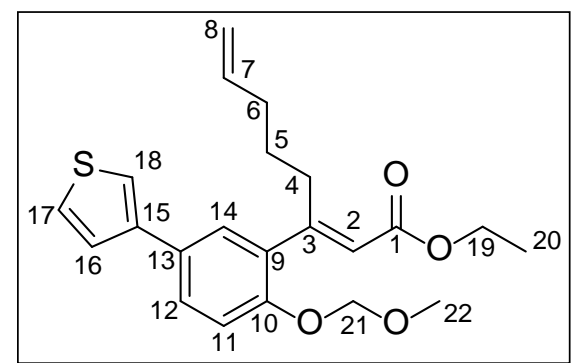

Thiophene-3-boronic acid, pinacol ester (0.42 g, 2 mmol, 1.14 eq.), palladium acetate (19.6 mg, $0.0875 \mathrm{mmol}, 0.05$ eq.), SPhos (72 mg, $0.175 \mathrm{mmol}, 0.1$ eq.) and $\mathrm{K}_{3} \mathrm{PO}_{4}(1.11$ g, 5.25 mmol, 3 eq.) were weighed into an oven dried Schlenk tube under an argon atmosphere. The tube was sealed and purged with argon before the addition of aryl bromide S-8 (0.68 g, $1.75 \mathrm{mmol}$, 1eq.) as a solution in dry dioxane ( $8 \mathrm{~mL})$. The reaction mixture was stirred at $80{ }^{\circ} \mathrm{C}$ for 15 hours. After completion, the reaction mixture was cooled to RT, diluted with $\mathrm{CH}_{2} \mathrm{Cl}_{2}(50 \mathrm{~mL})$, filtered and the filtrate was concentrated in vacuo. The crude residue was purified via column chromatography $\left(\mathrm{SiO}_{2}, n\right.$-pentane:diethyl ether $\left.=12: 1\right)$ to provide the product as a thick colorless oil (390 mg, $57 \%$ ).

$\mathbf{R}_{\boldsymbol{f}}=0.69$ (n-pentane:diethyl ether = 7:3); ${ }^{\mathbf{1}} \mathbf{H}$ NMR (500 MHz, Chloroform- $d$ ) $\delta 7.51-$ 7.47 (m, 1H; H12), 7.39 - 7.36 (m, 2H; H16, H18), 7.35 - 7.31 (m, 2H; H14, H17), 7.15 (d, J = $8.6 \mathrm{~Hz}, 1 \mathrm{H} ; \mathrm{H} 11), 5.87$ (t, $J=0.7 \mathrm{~Hz}, 1 \mathrm{H} ; \mathrm{H} 2), 5.76$ (ddt, $J=16.9,10.2,6.7 \mathrm{~Hz}, 1 \mathrm{H} ; \mathrm{H} 7$ ), 5.19 (s, 2H; H21), 5.00 - 4.87 (m, 2H; H8), 4.22 (q, $J=7.2$ Hz, 2H; H17), 3.48 (s, 3H; H22), 3.15 - 3.05 (m, 2H; H4), 2.13 - 2.03 (m, 2H; H6), $1.54-1.42$ (m, 2H; H5), 1.32 (t, J = 7.1 Hz, 3H; H20); ${ }^{13}$ C NMR (126 MHz, Chloroform-d) $\delta 166.5$ (C1), 160.7 (C3), 153.3 (C10), 141.6 (C15), 138.7 (C7), 132.9 (C13), 129.9 (C9), 127.7 (C14), 127.4 (C12), 126.40 (C16), 126.37 (C17), 119.90 (C2), 119.69 (C18), 115.0 (C11), 114.7 (C8), 94.7 (C21), 60.0 (C19), 56.4 (C22), 34.0 (C5), 32.0 (C4), 27.9 (C6), 14.5 (C20); HR-ESI-MS: m/z 409.1447 ([M+Na] $]^{+}$, calcd. for $\mathrm{C}_{22} \mathrm{H}_{26} \mathrm{O}_{4} \mathrm{SNa}^{+}$: 409.1444); IR (ATR): $\tilde{v}=2848$ (w), 1724 (s), 1600 (w), 1475 (m), 1451 (m), 1433 (m), 1395 (w), 1321 (m), 1238 (s), 1136 (m), 1091 (m), 1054 (m), 1018 (w), 969 (w), 912 (w), $844(\mathrm{~m}), 781(\mathrm{w}), 757(\mathrm{~s}) \mathrm{cm}^{-1}$

\section{Ethyl (E)-3-(2-hydroxy-5-(thiophen-3-yl)phenyl)octa-2,7-dienoate (S-11)}

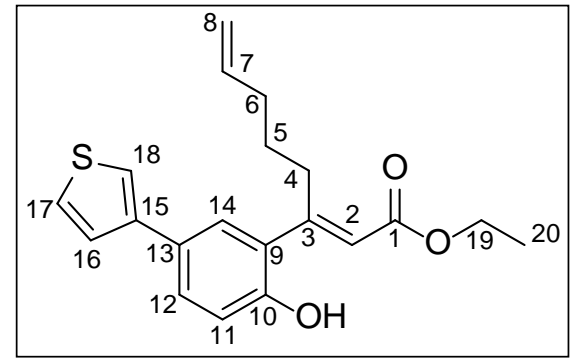

To a solution of compound S-10 (58 $\mathrm{mg}, 0.15 \mathrm{mmol}$, 1eq.) in dry $\mathrm{CH}_{2} \mathrm{Cl}_{2}(1.5 \mathrm{~mL})$, trimethylsilyl bromide (46 mg, 0.3 mmol, 2 eq.) was added. The reaction mixture was stirred at RT for 2 hours before the addition of ethanol ( $1 \mathrm{~mL})$ and the reaction mixture was concentrated in vacuo. After purification of the crude product with column chromatography $\left(\mathrm{SiO}_{2}, n\right.$-pentane:diethyl ether = 9:1), a colorless oil was obtained (152 mg, 90\%). 
$\mathbf{R}_{\boldsymbol{f}}=0.45$ (n-pentane:diethyl ether = 7:3); ${ }^{1} \mathbf{H}$ NMR (500 MHz, Chloroform- $d$ ) $\delta 7.45$ (dd, $J=8.4,2.3 \mathrm{~Hz}, 1 \mathrm{H}$; H12), 7.38 (dd, $J=4.9,3.0 \mathrm{~Hz}, 1 \mathrm{H}$ ), 7.34 (dd, $J=3.0,1.4 \mathrm{~Hz}, 1 \mathrm{H}$ ), 7.33 - 7.31 (m, 2H), 6.92 (d, $J=8.4 \mathrm{~Hz}, 1 \mathrm{H}$; H11), 5.96 (d, $J=0.7 \mathrm{~Hz}, 1 \mathrm{H}$ ), 5.76 (ddt, $J=17.0$, 10.2, $6.7 \mathrm{~Hz}, 1 \mathrm{H}$ ), 5.20 (s, 1H), $5.01-4.89$ (m, 2H), 4.23 (q, $J=7.1 \mathrm{~Hz}, 2 \mathrm{H}), 3.10-3.03$ (m, 2H), $2.13-2.05$ (m, 2H), 1.53 (dtd, $J=10.4,7.7,5.5 \mathrm{~Hz}, 2 \mathrm{H}), 1.32$ (t, $J=7.1 \mathrm{~Hz}, 3 \mathrm{H}) ;{ }^{13} \mathrm{C}$ NMR (126 MHz, Chloroform-d) $\delta 166.0$ (C1), 158.3 (C3), 151.5 (C10), 141.7 (C15), 138.4 (C7), 129.09 (C9), 128.97 (C13), 127.8 (C14), 126.6 (C16), 126.4 (C17), 126.3 (C12), 120.7 (C18), 119.5 (C2), 116.6 (C11), 115.1 (C8), 60.3 (C19), 34.0 (C5), 32.7 (C4), 27.9 (C6), 14.4 (C20); HR-ESI-MS: m/z 365.1190 ([M+Na $]^{+}$, calcd. for $\mathrm{C}_{20} \mathrm{H}_{22} \mathrm{O}_{3} \mathrm{SNa}^{+}$: 365.1187); IR (ATR): $\tilde{v}=2871$ (m), 1711 (s), 1601 (w), 1566 (w), 1433 (m), 1409 (m), 1289 (s), 1246 (s), 1187 (m), 1124 (m), 1091 (s), 1057 (m), 1026 (m), 811 (m), 712 (s), 671 (w) cm .

Ethyl (E)-3-(4-(methoxymethoxy)-4'-methyl-[1,1'-biphenyl]-3-yl)octa-2,7-dienoate (S-12)

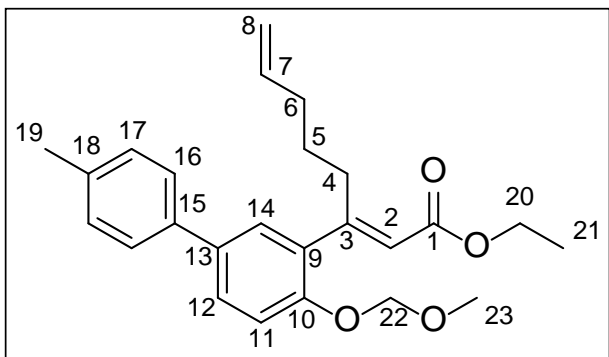

Toluene-4-boronic acid (0.27 g, $2 \mathrm{mmol}, 1.14$ eq.), palladium acetate (19.6 mg, $0.0875 \mathrm{mmol}, 0.05$ eq.), SPhos (72 mg, 0.175 mmol, 0.1 eq.) and $\mathrm{K}_{3} \mathrm{PO}_{4}$ (1.11 g, $5.25 \mathrm{mmol}, 3$ eq.) were weighed into an oven dried Schlenk tube under argon atmosphere. The tube was sealed and purged with argon before the addition of aryl bromide S-8 (0.68 g, $1.75 \mathrm{mmol}$, 1eq.) as a solution in dry dioxane $(8 \mathrm{~mL})$. The reaction mixture was stirred at $80{ }^{\circ} \mathrm{C}$ for 15 hours. After completion, the reaction mixture was cooled to RT, diluted with $\mathrm{CH}_{2} \mathrm{Cl}_{2}(50 \mathrm{~mL})$, filtered and the filtrate was concentrated in vacuo. The crude mixture was purified via column chromatography $\left(\mathrm{SiO}_{2}, n\right.$-pentane:diethyl ether $\left.=9: 1\right)$ to provide product as a thick colorless oil (435 mg, 63\%).

$\mathbf{R}_{\boldsymbol{f}}=0.70$ (n-pentane:diethyl ether $=7: 3$ ); ${ }^{\mathbf{1}} \mathbf{H}$ NMR (500 MHz, Chloroform- $d$ ) $\delta 7.48$ (dd, $J=8.5,2.4$ Hz, 1H; H12), 7.46 - 7.42 (m, 2H; H16), 7.32 (d, $J=2.4$ Hz, 1H; H14), 7.25 - 7.21 (m, 2H; H17), 7.17 (d, $J=8.6$ Hz, 1H; H11), 5.89 (s, 1H; H2), 5.76 (ddt, $J=17.0$, 10.2, 6.7 Hz, 1H; H7), 5.20 (s, 2H; H22), 4.99 - 4.88 (m, 2H; H8), 4.22 (q, $J=7.1 \mathrm{~Hz}, 2 \mathrm{H} ; \mathrm{H} 20$ ), 3.48 (s, 3H; H23), 3.15 - 3.08 (m, 2H; H4), 2.39 (s, 3H; H19), 2.14 - 2.04 (m, 2H; H6), 1.54 - 1.44 (m, 2H; H5), 1.32 (t, $J=7.1 \mathrm{~Hz}, 3 \mathrm{H} ; \mathrm{H} 21) ;{ }^{13} \mathrm{C}$ NMR (126 MHz, Chloroform-d) $\delta 166.5$ (C1), 160.8 (C3), 153.3 (C10), 138.7 (C7), 137.5 (C15), 136.9(C18), 134.9 (C13), 132.9 (C9), 129.6 (C17), 128.2 (C14), 127.8 (C12), 126.8 (C16), 119.9 (C2), 115.0 (C11), 114.7 (C8), 94.7 (C22), 60.0 (C20), 56.4 (C23), 34.0 (C5), 32.0 (C4), 27.9 (C6), 21.2 (C21), 14.5 (C21); 
HR-ESI-MS: $\mathrm{m} / \mathrm{z} 417.2054\left([\mathrm{M}+\mathrm{Na}]^{+}\right.$, calcd. for $\mathrm{C}_{25} \mathrm{H}_{30} \mathrm{O}_{4} \mathrm{Na}^{+}:$417.2036); IR (ATR): $\tilde{v}=$ 2980 (w), 2893 (w), 1709 (s), 1626 (m), 1514 (w), 1464 (w), 1381 (w), 1217 (w), 1154 (s), 1096 (m), 1078 (w), $1018(\mathrm{~m}), 923(\mathrm{w}), 873$ (m), $736(\mathrm{w}) \mathrm{cm}^{-1}$.

\section{Ethyl (E)-3-(4-hydroxy-4'-methyl-[1,1'-biphenyl]-3-yl)octa-2,7-dienoate (S-13)}

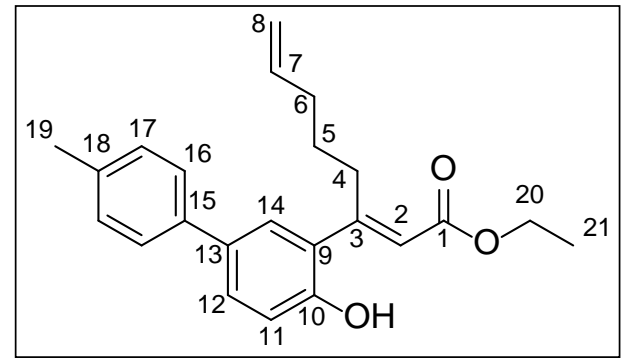

To a solution of compound S-12 (79 mg, $0.2 \mathrm{mmol}$, 1eq.) in dry $\mathrm{CH}_{2} \mathrm{Cl}_{2}(2 \mathrm{~mL})$, trimethylsilyl bromide (61 mg, 0.4 mmol, 2 eq.) was added. The reaction mixture was stirred at RT for 2 hours before the addition of ethanol $(1 \mathrm{~mL})$ and the reaction mixture was concentrated in vacuo. After purification of the crude product with column chromatography $\left(\mathrm{SiO}_{2}, n\right.$-pentane:diethyl ether $=9: 1)$, a colorless oil was obtained (39 mg, 56\%).

$\mathbf{R}_{\boldsymbol{f}}=0.20$ (n-pentane:diethyl ether $\left.=7: 3\right) ;{ }^{\mathbf{1}} \mathbf{H}$ NMR $(600 \mathrm{MHz}$, Chloroform- $d$ ) $\delta 7.43$ (dd, $J=8.3$, 2.3 Hz, 3H; H12, H16), 7.30 (d, $J=2.3 \mathrm{~Hz}, 1 \mathrm{H}$; H14), 7.24 - 7.21 (m, 2H; H17), 6.95 (d, $J=8.4 \mathrm{~Hz}, 1 \mathrm{H} ; \mathrm{H} 11$ ), 5.98 (t, $J=0.7 \mathrm{~Hz}, 1 \mathrm{H}$; H2), 5.76 (ddt, $J=17.0,10.2,6.7 \mathrm{~Hz}, 1 \mathrm{H}$; H7), 5.22 (s, 1H; OH), 5.00 - 4.89 (m, 2H; H8), 4.23 (q, J = 7.2 Hz, 2H; H20), 3.10 - 3.05 (m, 2H; H4), 2.39 (s, 3H; H19), 2.12 - 2.05 (m, 2H; H6), 1.57 - 1.49 (m, 2H; H5), 1.32 (t, J = 7.2 Hz, 3H; H21); ${ }^{13}$ C NMR (151 MHz, Chloroform-d) $\delta 165.9$ (C1), 158.3 (C3), 151.4 (C10), 138.2 (C7), 137.4 (C15), 136.7 (C18), 133.8 (C13), 129.7 (C17), 128.9 (C9), 128.0 (C12), 126.9 (C14), 126.5 (C16), 120.4 (C2), 116.4 (C11), 114.9 (C7), 60.1 (C20), 33.8 (C5), 32.5 (C4), 27.7 (C6), 21.0 (C19), 14.3 (C21). HR-ESI-MS: m/z 373.1770 ([M+Na] $]^{+}$, calcd. for $\mathrm{C}_{23} \mathrm{H}_{26} \mathrm{O}_{3} \mathrm{Na}^{+}$: 373.1774); IR (ATR): $\tilde{v}$ = 2983 (w), 1715 (s), 1626 (m), 1610 (m), 1510 (w), 1443 (w), 1376 (w), 1365 (m), 1341 (m), 1274 (m), 1217 (w), 1078 (w), 1042 (m), 1018 (m), 923 (w), 873 (m), $732(\mathrm{w}) \mathrm{cm}^{-1}$.

\section{2-Hydroxy-4-(trifluoromethyl)benzaldehyde (S-14)}

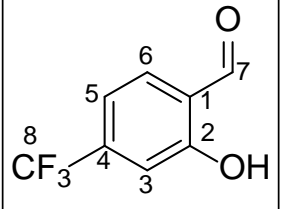

Hexamethylenetetramine (10.51 g, $75 \mathrm{mmol}, 3 \mathrm{eq}$ ) was added portionwise to a solution of (3-trifluoromethyl) phenol (4.05 g. $25 \mathrm{mmol}$, 1eq.) in trifluoroacetic acid $(25 \mathrm{~mL})$. The solution was heated to $80{ }^{\circ} \mathrm{C}$ for 8 hours and then stirred for additional 48 hours at RT. After completion, the reaction mixture was diluted with hydrochloric acid ( $25 \mathrm{~mL}, 6 \mathrm{M})$ and stirred for one hour. The $\mathrm{pH}$ of the mixture was than adjusted to 5 with a solution of sodium hydroxide (10\%). The aqueous phase was 
then extracted with diethyl ether $\left(3 \times 50 \mathrm{~mL}\right.$ ). Organics were dried over $\mathrm{MgSO}_{4}$ and concentrated in vacuo. After purification of crude product with column chromatography $\left(\mathrm{SiO}_{2}\right.$, $n$-pentane:diethyl ether $=9: 1)$ colorless oil was obtained $(1.07 \mathrm{~g}, 90 \%)$.

$\mathbf{R}_{\boldsymbol{f}}=0.52$ (n-pentane:diethyl ether = 9:1); ${ }^{1} \mathbf{H}$ NMR (400 MHz, Chloroform- $d$ ) $\delta 11.07$ (s, 1H; OH), 9.99 (d, $J=0.6 \mathrm{~Hz}, 1 \mathrm{H}$; H7), 7.71 (dt, $J=8.4,0.9 \mathrm{~Hz}, 1 \mathrm{H} ; \mathrm{H} 5), 7.37$ - 7.19 (m, 2H; H3, H6); ${ }^{13}$ C NMR (101 MHz, Chloroform-d) $\delta 196.3$ (C7), 161.6 (C2), 138.1 (q, $J=33.0$ Hz; C4), 134.4 (C6), 123.1 (q, $J=273.4$ Hz; C8), 122.4 (C1), 116.5 (q, $J=3.6 \mathrm{~Hz}$; C5), 115.5 (q, $J=3.9 \mathrm{~Hz}$; C3); ${ }^{19}$ F NMR (376 MHz, Chloroform- $d$ ) $\delta$-63.89; HR-ESI-MS: m/z 245.0409 ([M+Na+ $\left.\mathrm{CH}_{3} \mathrm{OH}\right]^{+}$, calcd. for $\left(\mathrm{C}_{8} \mathrm{H}_{5} \mathrm{O}_{2} \mathrm{~F}_{3} \mathrm{NaCH}_{3} \mathrm{OH}\right)^{+}:$245.0396); IR (ATR): $\tilde{v}=3380$ (br), 2845 (w), 1710 (s), 1650 (m), 1478 (w), 1420 (m), 1142 (m), 1097 (w), 1003 (m), 923 (m), 860 (w) $\mathrm{cm}^{-1}$.

\section{2-(Methoxymethoxy)-4-(trifluoromethyl)benzaldehyde (S-15)}<smiles>O=Cc1ccc(C(F)(F)F)cc1OCOCCCCCC(F)(F)F</smiles>

To a mixture of aldehyde $\mathbf{S - 1 4}$ (1.07g, $5.6 \mathrm{mmol}$, 1eq.) and potassium carbonate (1.55 g, $11.2 \mathrm{mmol}$, 2eq.) in acetonitrile (12 mL) methoxymethyl chloride $(0.54 \mathrm{~g}, 6.72 \mathrm{mmol}, 1.2$ eq.) was added. The reaction mixture was stirred at RT for 1 hour. After completion, the with a solution of sodium hydroxide $(0.5 \mathrm{M}, 20 \mathrm{~mL})$. Organics were separated, dried over $\mathrm{MgSO}_{4}$ and concentrated in vacuo. After purification of the crude product with column chromatography $\left(\mathrm{SiO}_{2}, n\right.$-pentane:diethyl ether = 9:1), a colorless oil was obtained (1.07 $\mathrm{g}$, $81 \%)$.

$\mathbf{R}_{\boldsymbol{f}}=0.31$ (n-pentane:diethyl ether = 9:1); ${ }^{1} \mathbf{H}$ NMR (400 MHz, Chloroform- $d$ ) $\delta 10.52(\mathrm{~d}$, $J=0.8 \mathrm{~Hz}, 1 \mathrm{H}$; H7), 7.97 - 7.90 (m, 1H; H5), 7.34 (ddt, $J=8.1,1.5,0.8 \mathrm{~Hz}, 1 \mathrm{H} ; \mathrm{H6}$ ), 7.26 (s, 1H; H3), 5.35 (s, 2H; H8), 3.55 (s, 3H; H9); ${ }^{13}$ C NMR (151 MHz, Chloroform-d) $\delta 188.93$ (C7), 159.65 (C2), 137.01 (q, $J=32.7$ Hz; C4), 129.18 (C6), 127.72 (C1), 123.39 (q, $J=273.3$ Hz; C10), 118.66 (q, $J=3.8$ Hz; C5), 112.54 (q, $J=3.8 \mathrm{~Hz}$; C3), 95.09 (C8), 56.88 (C9); ${ }^{19}$ F NMR (376 MHz, Chloroform-d) $\delta$-63.36; HR-ESI-MS: m/z 257.0402 ([M+Na] $]^{+}$, calcd. for $\mathrm{C}_{10} \mathrm{H}_{9} \mathrm{O}_{3} \mathrm{~F}_{3} \mathrm{Na}^{+}$: 257.0396); IR (ATR): $\tilde{v}=2871$ (w), 1678 (s), 1610 (w), 1471 (m), 1410 (w), $1258(\mathrm{~m}), 1198(\mathrm{w}), 1069(\mathrm{~m}), 974(\mathrm{~m}), 841(\mathrm{w}) \mathrm{cm}^{-1}$. 


\section{1-(2-(Methoxymethoxy)-4-(trifluoromethyl)phenyl)hex-5-en-1-ol (S-16)}

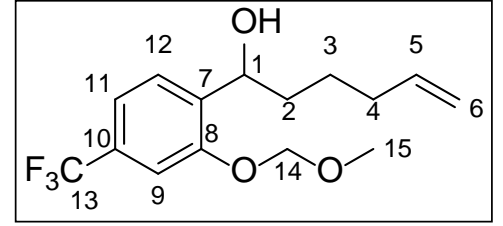

An oven dried Schlenk tube under argon atmosphere was charged with magnesium (0.25 g, $10.3 \mathrm{mmol}, 2.3 \mathrm{eq})$, catalytic iodine and heated with a heat gun until vapors of iodine appeared. After 5 minutes dry THF (7 mL) was added followed by 5-bromopent-1-ene ( $0.77 \mathrm{~g}, 5.16 \mathrm{mmol}, 1.16$ eq.). The reaction mixture was stirred for 1 hour at RT before the addition of a solution of aldehyde S-15 (1.03 g, $4.4 \mathrm{mmol}, 1$ eq.) in THF (7 mL). The reaction mixture was stirred for an additional 30 minutes and was then quenched with saturated ammonium chloride solution (5 mL) and diluted with $\mathrm{CH}_{2} \mathrm{Cl}_{2}$ (50 ml). The organic layer was washed with water $(50 \mathrm{~mL})$, dried over $\mathrm{MgSO}_{4}$ and concentrated in vacuo. After purification of the crude product with column chromatography $\left(\mathrm{SiO}_{2}, n\right.$-pentane:diethyl ether $=4: 1)$, a colorless oil was obtained $(0.76 \mathrm{~g}, 56 \%)$.

$\mathbf{R}_{\boldsymbol{f}}=0.28$ (n-pentane:diethyl ether $\left.=4: 1\right) ;{ }^{1} \mathbf{H}$ NMR $(600 \mathrm{MHz}$, Chloroform- $d) \delta 7.50$ (d, $J$ = $7.9 \mathrm{~Hz}, 1 \mathrm{H} ; \mathrm{H} 12), 7.34$ - 7.31 (m, 1H; H9), 7.28 (dd, $J=7.9,1.7 \mathrm{~Hz}, 1 \mathrm{H} ; \mathrm{H} 11), 5.79$ (ddt, $J$ = 16.9, 10.2, $6.6 \mathrm{~Hz}, 1 \mathrm{H}$; H5), 5.25 (s, 2H; H14), 5.04 - 4.93 (m, 3H; H1, H6), 3.50 (s, 3H; H15), 2.10 (dtd, $J=8.1,6.8,1.4$ Hz, 2H; H4), 1.81 - 1.74 (m, 2H; H2), 1.62 - 1.42 (m, 2H; H3); ${ }^{13}$ C NMR (151 MHz, Chloroform-d) $\delta 154.2$ (C8), 138.7 (C5), 137.5 (C7), 130.7 (q, $J=$ 32.5 Hz; C10), 127.3 (C12), 123.2 (q, $J=272.0$ Hz; C13), 118.9 (q, $J=3.9$ Hz; C11), 114.9 (C8), 110.9 (q, $J=3.8$ Hz; C9), 94.7 (C14), 69.6 (C1), 56.6 (C15), 37.0 (C2), 33.7 (C3), 25.2 (C4); ${ }^{19}$ F NMR (564 MHz, Chloroform-d) $\delta$-62.44; HR-ESI-MS: m/z $327.1202\left([\mathrm{M}+\mathrm{Na}]^{+}\right.$, calcd. for $\mathrm{C}_{15} \mathrm{H}_{19} \mathrm{O}_{3} \mathrm{~F}_{3} \mathrm{Na}^{+}$: 327.1179); IR (ATR): v =3410 (br), 2980 (w), 1579 (w), 1438 (m), 1367 (w), 1344 (m), 1287 (w), 1244 (m), 1164 (s), 1132 (m), 1076 (w), 942 (w), 879 (m), 821 (m), $741(\mathrm{~s}) \mathrm{cm}^{-1}$.

\section{1-(2-(Methoxymethoxy)-4-(trifluoromethyl)phenyl)hex-5-en-1-one (S-17)}

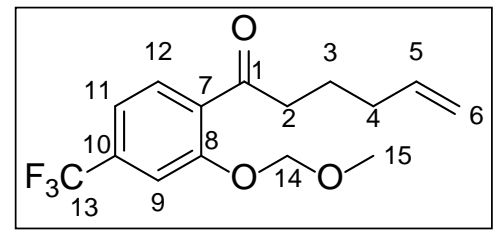

To a solution of alcohol S-16 (0.73 g, $2.41 \mathrm{mmol}, 1$ eq.) in $\mathrm{CH}_{2} \mathrm{Cl}_{2}(22 \mathrm{~mL})$ pyridinium chlorochromate $(0.68 \mathrm{~g}, 3.13 \mathrm{mmol}$, 1.3 eq.) was added. Reaction mixture was stirred for 1 hour and then it was diluted with $n$-pentane $(22 \mathrm{~mL})$. Solids were removed by filtration and the filtrate was concentrated in vacuo. After purification of crude product with column chromatography ( $\mathrm{SiO}_{2}, n$-pentane:diethyl ether =9:1) colorless oil was obtained $(0.63$ g, 87\%). 
$\mathbf{R}_{\boldsymbol{f}}=0.44$ (n-pentane:diethyl ether $\left.=9: 1\right)$; ${ }^{1} \mathbf{H}$ NMR (600 MHz, Chloroform- $d$ ) $\delta 7.69-$ 7.63 (m, 1H; H11), 7.43 (dd, $J=1.7,0.8 \mathrm{~Hz}, 1 \mathrm{H}$; H9), 7.30 (ddt, $J=8.0,1.2,0.6 \mathrm{~Hz}, 1 \mathrm{H}$; H12), 5.80 (ddt, $J=16.9,10.2,6.7$ Hz, 1H; H5), 5.29 (s, 2H; H14), 5.06 - 4.94 (m, 2H; H6), 3.52 (s, 3H; H15), 3.00 - 2.93 (m, 2H; H2), 2.17 - 2.09 (m, 2H; H4), 1.81 (p, J = 7.4 Hz, 2H; H3); ${ }^{13} \mathrm{C}$ NMR (151 MHz, Chloroform-d) $\delta 202.5$ (C1), 155.7 (C8), 138.2 (C5), 134.5 (q, $J=32.5$ Hz; C10)), 132.9 (C7), 130.34 (C12), 123.6 (q, $J=272.9 \mathrm{~Hz}$; C13), 118.7 (q, $J=3.8 \mathrm{~Hz}$; C11), 115.4 (C6), 112.1 (q, $J=3.8$ Hz; C9), 94.9 (C14), 56.8 (C15), 43.1 (C2), 33.3 (C3), 23.4 (C4); ${ }^{19}$ F NMR (564 MHz, Chloroform- $d$ ) $\delta$-63.05; HR-ESI-MS: m/z 325.1045 ([M+Na $]^{+}$, calcd. for $\mathrm{C}_{15} \mathrm{H}_{17} \mathrm{O}_{3} \mathrm{~F}_{3} \mathrm{Na}^{+}$: 325.1022); IR (ATR): $\tilde{v}=2983$ (w), 1683 (s), 1547 (w), 1489 (m), 1447 (m), 1231 (m), 1173 (s), 1162 (m), 1076 (w), 961 (w), 878 (m), 824 (m), 755 (s) cm².

Ethyl (E)-3-(2-(methoxymethoxy)-4-(trifluoromethyl)phenyl)octa-2,7-dienoate (S-18)

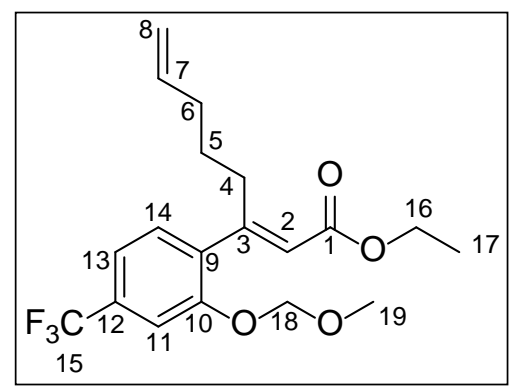

Sodium hydride (160 mg, 4 mmol, 2 eq.; 60 \% in paraffin oil) was weighed into an oven dried Schlenk tube under argon atmosphere. The flask was sealed before the addition of THF (6 $\mathrm{mL})$ and dropwise addition of triethyl phosphonoacetate $(0.90$ g, $4 \mathrm{mmol}, 2$ eq.). Once the evolution of hydrogen stopped, a solution of ketone S-17 (0.61 g, 2 mmol, 1 eq.) in THF (3 mL) was added. The reaction mixture was stirred at RT for 16 hours and was quenched with saturated ammonium chloride solution $(5 \mathrm{~mL})$. The reaction was diluted with $\mathrm{CH}_{2} \mathrm{Cl}_{2}(25 \mathrm{~mL})$ and organics were washed with water $(20 \mathrm{~mL})$ and concentrated in vacuo. After purification of the crude product with column chromatography $\left(\mathrm{SiO}_{2}, n\right.$-pentane:diethyl ether $\left.=30: 1\right)$, a colorless oil was obtained $(0.47 \mathrm{~g}, 63 \%)$.

$\mathbf{R}_{\boldsymbol{f}}=0.14$ (n-pentane:diethyl ether $\left.=30: 1\right)$; ${ }^{1} \mathbf{H}$ NMR (500 MHz, Chloroform- $d$ ) $\delta 7.36$ (d, $J=1.6 \mathrm{~Hz}, 1 \mathrm{H}$; H14), 7.27 - 7.23 (m, 1H; H13), 7.20 (dd, $J=7.9,0.9 \mathrm{~Hz}, 1 \mathrm{H} ; \mathrm{H} 11$ ), 5.80 (s, 1H; H2), 5.74 (ddt, $J=16.9,10.2,6.7$ Hz, 1H; H7), 5.21 (s, 2H; H18), 5.01 - 4.86 (m, 2H; H8), 4.21 (q, J = 7.1 Hz, 2H; H16), 3.47 (s, 3H; H19), 3.09 - 3.00 (m, 2H; H4), 2.09 - 2.00 (m, 2H; H6), 1.49 - 1.39 (m, 2H; H5), 1.31 (t, $J=7.1 \mathrm{~Hz}, 3 \mathrm{H}$; H17); ${ }^{13} \mathrm{C}$ NMR (126 MHz, Chloroformd) $\delta 166.2$ (C1), 159.1 (C3), 154.3 (C10), 138.5 (C7), 136.0 (C9), 131.6 (q, $J=32.6$ Hz; C12), 123.9 (q, $J=272.4$ Hz; C15), 123.0 (C14), 120.5 (C2), 118.6 (q, $J=3.9$ Hz; C13), 114.9 (C8), 111.6 (q, $J=3$ 3;.8 Hz; C11), 94.8 (C18), 60.2 (C16), 56.6 (C19), 33.9 (C5), 31.7 (C4), 27.7 (C6), 14.4 (C17); ${ }^{19}$ F NMR (376 MHz, Chloroform- $d$ ) $\delta$-62.54; HR-ESI-MS: m/z 395.1441 ([M+Na] $]^{+}$, calcd. for $\mathrm{C}_{19} \mathrm{H}_{23} \mathrm{O}_{4} \mathrm{~F}_{3} \mathrm{Na}^{+}$: 395.1441); IR (ATR): $\tilde{v}=2863$ (w), 1710 (s), 1634 (m), 
1447 (w), 1367 (w), 1314 (m), 1277 (w), 1245 (m), 1211 (m), 1089 (w), 1074 (w), 1041 (s), $923(\mathrm{w}), 871(\mathrm{~m}), 826(\mathrm{~m}) \mathrm{cm}^{-1}$.

\section{Ethyl (E)-3-(2-hydroxy-4-(trifluoromethyl)phenyl)octa-2,7-dienoate (S-19)}

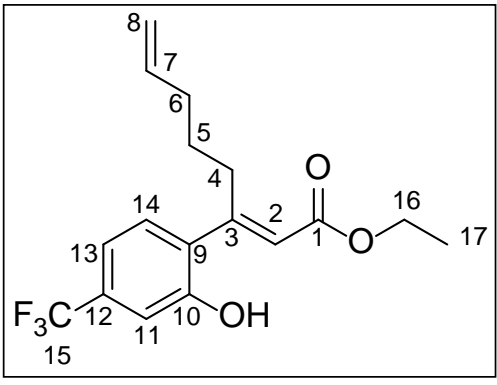

To the solution of compound S-18 (150 mg, $0.4 \mathrm{mmol}$, 1eq.) in dry $\mathrm{CH}_{2} \mathrm{Cl}_{2}(1.5 \mathrm{~mL})$, trimethylsilyl bromide (122 $\mathrm{mg}, 0.8$ mmol, 2 eq.) was added. The reaction mixture was stirred at RT for 2 hours before the addition of ethanol $(2 \mathrm{~mL})$ and the reaction mixture was concentrated in vacuo. After purification of the crude product with column chromatography $\left(\mathrm{SiO}_{2}, n-\right.$ pentane:diethyl ether =12:1), a colorless oil was obtained (126 mg, 95\%).

$\mathbf{R}_{\boldsymbol{f}}=0.18$ (n-pentane:diethyl ether $\left.=12: 1\right) ;{ }^{1} \mathbf{H}$ NMR (400 MHz, Chloroform- $\left.d\right) \delta 7.23-$ 7.18 (m, 2H; H13, H14), 7.17 - 7.14 (m, 1H; H11), 5.92 (s, 1H; H2), 5.74 (ddt, $J$ = 16.9, 10.2, 6.7 Hz, 1H; H7), 5.60 (s, 1H; OH), $5.02-4.87$ (m, 2H; H8), 4.23 (q, J = 7.1 Hz, 2H; H16), 3.05 - 2.99 (m, 2H; H4), 2.13 - 1.96 (m, 2H; H6), 1.54 - 1.39 (m, 2H; H5), 1.32 (t, $J=7.1 \mathrm{~Hz}$, 3H; H17); ${ }^{13}$ C NMR (101 MHz, Chloroform-d) $\delta 165.9$ (C1), 157.0 (C3), 152.5 (C10), 138.1 (C7), 132.3 (C9), 131.8 (q, $J=32.7$ Hz; C12), 129.3 (C14), 123.8 (q, $J=272.3$ Hz; C15), 121.3 (C2), 117.5 (q, $J=3.9$ Hz; C13), 115.2 (C8), 113.3 (q, $J=3.9$ Hz; C11), 60.6 (C16), 33.9 (C5), 32.4 (C4), 27.7 (C6), 14.4 (C17); ${ }^{19}$ F NMR (376 MHz, Chloroform- $d$ ) $\delta$-62.87; HR-ESI-MS: m/z 351.1195 ([M+Na] $]^{+}$, calcd. for $\mathrm{C}_{17} \mathrm{H}_{19} \mathrm{O}_{3} \mathrm{~F}_{3} \mathrm{Na}^{+}$: 351.1179$)$ ); IR (ATR): $\tilde{v}=3321$ (br), 2977 (w), 1741 (s), 1655 (m), 1611 (m), 1588 (m), 1272 (w), 1234 (m), 1142 (m), 1074 (w), 1031 (m), 914 (w), 878 (m), 776 (m) $\mathrm{cm}^{-1}$.

\section{Ethyl (E)-3-(2-(methoxymethoxy)phenyl)-2-methylocta-2,7-dienoate (S-20)}

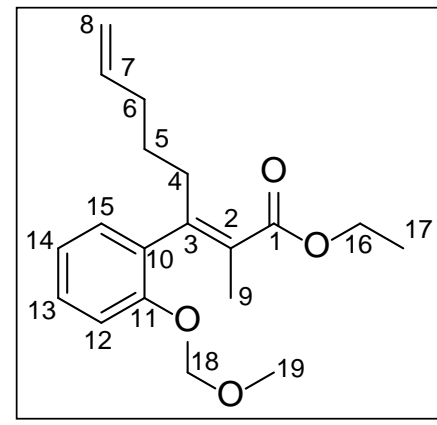

was quenched with saturated ammonium chloride solution $(5 \mathrm{~mL})$, diluted with water $(25 \mathrm{~mL})$ and extracted with $\mathrm{CH}_{2} \mathrm{Cl}_{2}\left(2\right.$ x $20 \mathrm{~mL}$ ). Organics were dried over $\mathrm{MgSO}_{4}$ and concentrated in

To a solution of triethyl 2-phosphonopropionate $(1.07 \mathrm{~g}, 4.5 \mathrm{mmol}$, 1.5 eq.) in THF $(6 \mathrm{~mL})$ cooled to $-10^{\circ} \mathrm{C}, n$-BuLi $(2.81 \mathrm{~mL}, 4.5$ mmol, 1.5 eq., in hexanes, $1.6 \mathrm{M}$ ) was added dropwise. The reaction mixture was stirred for 15 minutes before the dropwise addition of ketone S-3 (0.70 g, 3 mmol, 1eq.). The reaction mixture was then heated to $50{ }^{\circ} \mathrm{C}$ for 16 hours. After completion, the reaction mixture, 
vacuo. After purification of the crude product with column chromatography $\left(\mathrm{SiO}_{2}, n\right.$ pentane:diethyl ether =7:3), a colorless oil was obtained (0.12 g, 13\%).

$\mathbf{R}_{\boldsymbol{f}}=0.34$ (n-pentane:diethyl ether $=7: 3$ ); ${ }^{1} \mathbf{H}$ NMR (500 MHz, Chloroform- $d$ ) $\delta 7.23-$ 7.16 (m, 1H; H13), 7.07 (dt, $J=7.8,0.6$ Hz, 1H; H12), 6.95 - 6.88 (m, 2H; H14, H15), 5.74 (ddt, $J=16.9,10.2,6.6 \mathrm{~Hz}, 1 \mathrm{H} ; \mathrm{H7}$ ), 5.14 (s, 2H; H18), 5.00 - 4.89 (m, 2H; H8), 3.79 (qd, $J=$ 7.2, 0.5 Hz, 2H; H16), 3.45 (d, $J=0.5$ Hz, 3H; H19), 2.46 (s, 2H; H4), 2.09 - 1.97 (m, 5H; H5, H9), 1.42 (b, 2H; H5), 0.78 (td, $J=7.1,0.5 \mathrm{~Hz}, 3 \mathrm{H}$; H3); ${ }^{13} \mathrm{C}$ NMR (126 MHz, Chloroform-d)

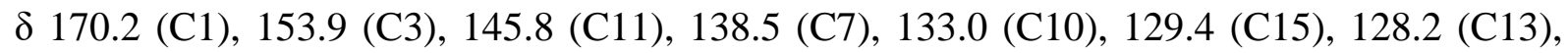
126.9 (C2), 121.5(C14), 114.9 (C8), 114.5 (C12), 94.6 (C18), 60.0 (C16), 56.1 (C19), 33.9 (C5), 33.8 (C4), 26.6 (C6), 15.7 (C9), 13.6 (C17); HR-ESI-MS: m/z 341.1724 ([M+Na]+, calcd. for $\mathrm{C}_{19} \mathrm{H}_{26} \mathrm{O}_{4} \mathrm{Na}^{+}$: 341.1729); IR (ATR): $\tilde{v}$ = 2971 (w), 2936 (w), 2834 (w), 1721 (s), 1630 (m), 1574 (w), 1511 (s), 1300 (m), 1255 (s), 1156 (m), 1067 (m), 1032 (s), 971 (w), 872 (m), 821 (s), $805(\mathrm{~m}), 733(\mathrm{w}) \mathrm{cm}^{-1}$.

\section{Ethyl (E)-3-(2-(hydroxy)phenyl)-2-methylocta-2,7-dienoate (S-21)}

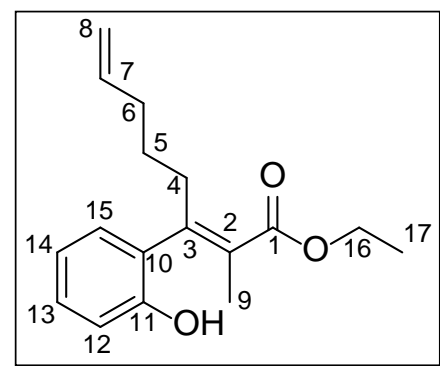

To a solution of compound S-20 (96 mg, $0.3 \mathrm{mmol}$, 1eq.) in dry $\mathrm{CH}_{2} \mathrm{Cl}_{2}$ (1.5 mL), trimethylsilyl bromide (92 mg, $0.6 \mathrm{mmol}, 2$ eq.) was added. The reaction mixture was stirred at RT for 2 hours before the addition of ethanol $(2 \mathrm{~mL})$ and the reaction mixture was concentrated in vacuo. After purification of crude product with column chromatography $\left(\mathrm{SiO}_{2}, n\right.$-pentane:diethyl ether $\left.=12: 1\right)$ colorless oil was obtained (75 mg, 91\%).

$\mathbf{R}_{\boldsymbol{f}}=0.21$ (n-pentane:diethyl ether = 10:1); ${ }^{1} \mathbf{H}$ NMR $(600 \mathrm{MHz}$, Chloroform- $d) \delta 7.55$ (dd, $J=8.0,1.5 \mathrm{~Hz}, 1 \mathrm{H}$; H15), 7.43 (ddd, $J=8.6,7.3,1.5 \mathrm{~Hz}, 1 \mathrm{H} ; \mathrm{H} 13$ ), 7.30 (dd, $J=8.3,1.2 \mathrm{~Hz}$, 1H; H12), 7.29 - 7.24 (m, 1H; H14), 6.78 (s, 1H; OH), 5.83 (ddt, $J=17.0,10.2,6.7 \mathrm{~Hz}, 1 \mathrm{H}$; H7), 5.13 - 5.03 (m, 2H; H8), 4.12 (q, J = 7.2 Hz, 2H; H16), 2.84 - 2.78 (m, 2H; H4), 2.27 2.19 (m, 5H; H6), 1.72 - 1.64 (m, 2H; H5), 1.33 (t, $J$ = 7.1 Hz, 3H; H17); ${ }^{13}$ C NMR (151 MHz, Chloroform-d) $\delta 162.3$ (C1), 152.6 (C3), 150.1 (C11), 137.6 (C7), 130.5 (C15), 124.32 (C13), 124.22 (C14), 122.1 (C2), 119.8 (C10), 117.2 (C12), 116.0 (C8), 61.9 (C16), 33.9 (C5), 28.2 (C4), 27.8 (C6), 16.5 (C9), 13.3 (C17); HR-ESI-MS: m/z 297.1478 ([M+Na] $]^{+}$, calcd. for $\mathrm{C}_{17} \mathrm{H}_{22} \mathrm{O}_{3} \mathrm{Na}^{+}$: 297.1467); IR (ATR): $\tilde{v}=3420$ (br), 2979 (w), 1720 (s), 1615 (m), 1569 (w), 1375 (m), 1273 (m), 1228 (s), 1212 (m), 1156 (s), 1113 (w), 1044 (s), 1031 (m), 946 (w), 724 (w), $670(\mathrm{w}) \mathrm{cm}^{-1}$. 


\section{1-(2-(Methoxymethoxy)phenyl)ethan-1-one (S-22)}

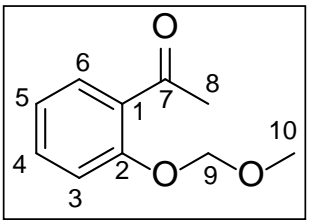

To a suspension of sodium hydride (0.63 g, $15.75 \mathrm{mmol}, 1.05$ eq., $60 \%$ in parafin oil) in THF (30 mL) cooled to - $10^{\circ} \mathrm{C}$, 1-(2-hydroxyphenyl)ethan1-one (2.04 g, 15 mmol, 1 eq.) was added. The reaction mixture was stirred for 30 minutes and then methoxymethyl chloride (1.45 g, $18 \mathrm{mmol}, 1.2 \mathrm{eq}$.) was added dropwise. The reaction mixture was stirred for 1 hour and was subsequently quenched with water (50 $\mathrm{mL})$. Organics were extracted with $\mathrm{CH}_{2} \mathrm{Cl}_{2}(2 \times 30 \mathrm{~mL})$, dried over $\mathrm{MgSO}_{4}$ and concentrated in vacuo. After purification of the crude product with column chromatography $\left(\mathrm{SiO}_{2}, n\right.$ pentane:diethyl ether =9:1), a colorless oil was obtained (2.17 g, 80\%).

$\mathbf{R}_{\boldsymbol{f}}=0.13$ ( $n$-pentane:diethyl ether = 9:1); ${ }^{1} \mathbf{H}$ NMR (300 MHz, Chloroform- $d$ ) $\delta 7.71$ (ddd, $J=7.7,1.9,0.4 \mathrm{~Hz}, 1 \mathrm{H} ; \mathrm{H6}$ ), 7.43 (ddd, $J=8.4,7.3,1.9 \mathrm{~Hz}, 1 \mathrm{H} ; \mathrm{H} 4$ ), 7.18 (dd, $J=8.4,1.1$ Hz, 1H; H3), 7.04 (ddd, $J$ = 7.7, 7.3, 1.0 Hz, 1H; H5), 5.28 (s, 2H; H9), 3.51 (s, 3H; H10), 2.64 (s, 3H; H8); ${ }^{13}$ C NMR (101 MHz, Chloroform-d) $\delta 200.0$ (C7), 156.3 (C2), 133.5 (C4), 130.2 (C6), 129.2 (C1), 121.7 (C5), 114.8 (C3), 94.5 (C9), 56.4 (C10), 31.8 (C8); HR-ESI-MS: m/z $203.0686\left([\mathrm{M}+\mathrm{Na}]^{+}\right.$, calcd. for $\mathrm{C}_{10} \mathrm{H}_{12} \mathrm{O}_{3} \mathrm{Na}^{+}$: 203.0684); IR (ATR): $\tilde{v}=2963(\mathrm{w}), 2871(\mathrm{w})$, 1668 (s), 1574 (w), 1530 (w), 1478 (m), 1397 (m), 1310 (w), 1063 (w), 965 (w), 741 (m) cm-1.

\section{2-Hydroxy-1-(2-(methoxymethoxy)phenyl)ethan-1-one (S-23)}

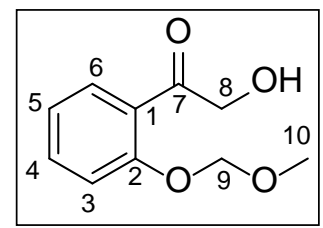

To a solution of potassium hydroxide ( $0.90 \mathrm{~g}, 16 \mathrm{mmol}, 8 \mathrm{eq}$.$) in methanol$ (30 mL), ketone S-22 (0.36 g, 2 mmol, 1 eq.) and bis(acetoxy)iodobenzene ( $0.97 \mathrm{~g}, 3 \mathrm{mmol}, 1.5 \mathrm{eq}$.) were added sequentially. The reaction mixture was stirred for 1 hour at $-10{ }^{\circ} \mathrm{C}$ and then at RT for an additional 14 hours. To the reaction mixture, diluted hydrochloric acid (30 mL, 1M) was added and with stirring for 30 minutes. The reaction mixture was diluted with water $(50 \mathrm{~mL})$ and organics were extracted with $\mathrm{CH}_{2} \mathrm{Cl}_{2}$ ( 2 x $30 \mathrm{~mL}$ ). Organics were dried over $\mathrm{MgSO}_{4}$ and concentrated in vacuo. After purification of the crude product with column chromatography $\left(\mathrm{SiO}_{2}, n\right.$-pentane:diethyl ether $\left.=7: 3\right)$, a colorless oil was obtained (0.28 g, 72\%).

$\mathbf{R}_{\boldsymbol{f}}=0.10$ (n-pentane:diethyl ether $=7: 3$ ); ${ }^{1} \mathbf{H}$ NMR (300 MHz, Chloroform- $d$ ) $\delta 8.05$ (dd, $J=7.9,1.8 \mathrm{~Hz}, 1 \mathrm{H} ; \mathrm{H} 6$ ), 7.53 (ddd, $J=8.5,7.2,1.9 \mathrm{~Hz}, 1 \mathrm{H} ; \mathrm{H} 4$ ), 7.22 (dd, $J=8.5,1.0 \mathrm{~Hz}$, 1H; H3), 7.11 (ddd, $J=8.1,7.2,1.0 \mathrm{~Hz}, 1 \mathrm{H}$; H5), 5.32 (s, 2H; H9), 4.82 (d, $J=4.7 \mathrm{~Hz}, 2 \mathrm{H}$; H8), 3.75 (t, $J=4.8 \mathrm{~Hz}, 1 \mathrm{H}$; OH), 3.52 (s, 3H; H10); ${ }^{13}$ C NMR (101 MHz, Chloroform-d) $\delta$ 199.2 (C7), 157.9 (C2), 135.4 (C4), 131.1 (C6), 123.7 (C1), 122.1 (C5), 114.8 (C3), 94.4 (C10), 
70.0 (C8), 56.8 (C9); HR-ESI-MS: m/z $219.0636\left([\mathrm{M}+\mathrm{Na}]^{+}\right.$, calcd. for $\mathrm{C}_{10} \mathrm{H}_{12} \mathrm{O}_{4} \mathrm{Na}^{+}$: 219.0628); IR (ATR): $\tilde{v}$ = 3511 (br), 2896 (w), 1736 (s), 1642 (m), 1541 (w), 1511 (w), 1413 (m), $1378(\mathrm{w}), 1236$ (m), $1160(\mathrm{w}), 967$ (m), $774(\mathrm{~m}) \mathrm{cm}^{-1}$.

\section{2-(Allyloxy)-1-(2-(methoxymethoxy)phenyl)ethan-1-one (S-24)}

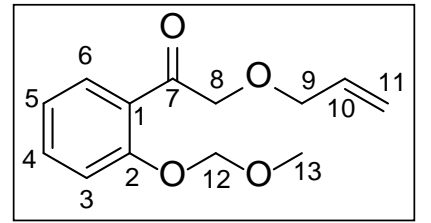

To a solution of compound S-23 (0.27 g, $1.5 \mathrm{mmol}$, 1eq.) in allyl bromide (3 mL) cooled to $-15{ }^{\circ} \mathrm{C}$ under argon atmosphere, silver oxide (1.04 g, $4.5 \mathrm{mmol}$, 3eq.) was added. After 1 hour the reaction mixture was allowed to warm to RT and was stirred for 14 hours. The reaction mixture was diluted with of $\mathrm{CH}_{2} \mathrm{Cl}_{2}(10 \mathrm{~mL})$ and solids were removed by filtration and the filtrate concentrated in vacuo. After purification of the crude product with column chromatography ( $\mathrm{SiO}_{2}, n$-pentane:diethyl ether $\left.=7: 3\right)$, a colorless oil was obtained (0.19 g, 53\%).

$\mathbf{R}_{\boldsymbol{f}}=0.30$ (n-pentane:diethyl ether $=7: 3$ ); ${ }^{1} \mathbf{H}$ NMR (500 MHz, Chloroform- $d$ ) $\delta 7.87-$ 7.81 (m, 1H, H6), 7.46 (ddd, $J=8.4,7.3,1.9$ Hz, 1H; H4), 7.17 (dd, $J=8.4,1.0$ Hz, 1H; H3), 7.11 - 7.04 (m, 1H; H5), 6.02 - 5.91 (m, 1H; H10), 5.33 - 5.28 (m, 1H; H11), 5.27 (s, 2H; H12), 5.23 - 5.19 (m, 1H; H11), 4.72 (s, 2H; H8), 4.15 (dt, $J$ = 5.8, 1.4 Hz, 2H; H9), 3.51 (s, 3H; H13); ${ }^{13}$ C NMR (126 MHz, Chloroform-d) $\delta 198.0$ (C7), 156.6 (C2), 134.4 (C10), 134.1 (C4), 130.5 (C6), 126.3 (C1), 122.0 (C5), 117.7 (C11), 114.6 (C3), 94.5 (C12), 76.2 (C8), 72.4 (C9), 56.5 (C13); HR-ESI-MS: m/z 259.0948 ([M+Na] $]^{+}$, calcd. for $\mathrm{C}_{13} \mathrm{H}_{16} \mathrm{O}_{4} \mathrm{Na}^{+}$: 259.0941); IR (ATR): $\tilde{v}=2923$ (w), 2889 (w), 1698 (s), 1630 (w), 1541 (m), 1423 (m), 1398 (w), 1341 (w), 1224 (m), 1187 (w), 1063 (m), 936 (m), 799 (m).

\section{Ethyl (E)-4-(allyloxy)-3-(2-(methoxymethoxy)phenyl)but-2-enoate (S-25)}

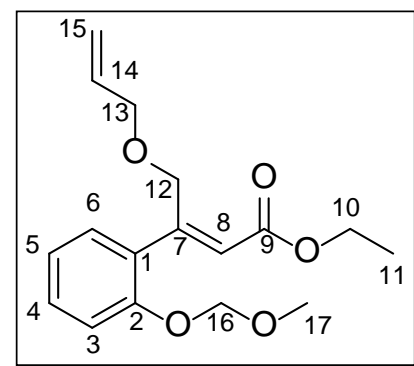

To a stirred solution of triethylphosphono acetate $(0.29 \mathrm{~g}, 1.3 \mathrm{mmol}$, 2eq.) in THF (2 mL) at $-78^{\circ} \mathrm{C}, n$-BuLi $(0.81 \mathrm{~mL}, 1.3 \mathrm{mmol}$, in hexanes, 1.6 M) was added dropwise. After one hour of stirring, ketone S-24 was added and the reaction mixture was warmed to RT and stirred for an additional 14 hours. After completion, the reaction

mixture was diluted with $\mathrm{CH}_{2} \mathrm{Cl}_{2}(20 \mathrm{~mL})$, washed with saturated ammonium chloride solution (20 mL), dried over $\mathrm{MgSO}_{4}$ and concentrated in vacuo. After purification of the crude product with column chromatography $\left(\mathrm{SiO}_{2}, n\right.$-pentane:diethyl ether $\left.=7: 3\right)$, a colorless oil was obtained (0.10 g, 50\%). 
$\mathbf{R}_{\boldsymbol{f}}=0.65$ (n-pentane:diethyl ether = 7:3); ${ }^{1} \mathbf{H}$ NMR (600 MHz, Chloroform- $d$ ) $\delta 7.28$ (ddd, $J=8.2,7.4,1.7$ Hz, 1H; H4), 7.22 - 7.19 (m, 1H; H6), 7.13 (dd, $J=8.3,1.1 \mathrm{~Hz}, 1 \mathrm{H} ; \mathrm{H3}$ ), 7.00 (td, $J=7.5,1.1 \mathrm{~Hz}, 1 \mathrm{H}$; H5), 5.96 (t, $J=1.5 \mathrm{~Hz}, 1 \mathrm{H}$; H8), 5.72 (ddt, $J=17.2,10.3,5.5 \mathrm{~Hz}$, 1H; H14), 5.17 (s, 2H; H16), 5.10 - 5.01 (m, 2H; H14), 4.95 (d, $J=1.5$ Hz, 2H; H12), 4.21 (q, $J=7.1 \mathrm{~Hz}, 2 \mathrm{H}$; H10), 3.87 (dt, $J=5.5,1.5 \mathrm{~Hz}, 2 \mathrm{H}$; H13), 3.47 (s, 3H; H17), 1.31 (t, $J=7.1$ Hz, 3H; H11); ${ }^{13}$ C NMR (151 MHz, Chloroform-d) $\delta 166.2$ (C9), 157.2 (C7), 154.8 (C2), 134.8 (C14), 130.1 (C1), 129.84 (C4), 129.83 (C6), 121.9 (C5), 120.8 (C8), 116.8 (C15), 114.6 (C3), 95.0 (C16), 71.5 (C13), 68.0 (C12), 60.4 (C10), 56.3 (C16), 14.4 (C11); HR-ESI-MS: m/z $329.1365\left([\mathrm{M}+\mathrm{Na}]^{+}\right.$, calcd. for $\mathrm{C}_{17} \mathrm{H}_{22} \mathrm{O}_{5} \mathrm{Na}^{+}$: 329.1359); IR (ATR): $\tilde{v}=2989(\mathrm{w}), 2901(\mathrm{w})$, 1725 (s), 1619 (w), 1569 (w), 1434 (w), 1405 (m), 1324 (s), 1283 (m), 1109 (s), 1091 (s), 1069 (s), 1049 (m), 1022 (m), 1007 (m), 963 (w), 845 (m), 796 (w), 766 (m), 670 (w) cm².

\section{Ethyl (Z)-4-(allyloxy)-3-(2-hydroxyphenyl)but-2-enoate (S-26)}

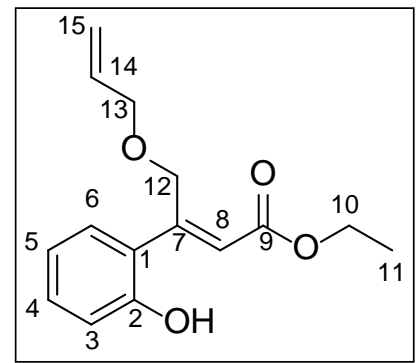

To a solution of compound S-25 (61 mg, $0.2 \mathrm{mmol}$, 1eq.) in dry $\mathrm{CH}_{2} \mathrm{Cl}_{2}$ (2 mL), trimethylsilyl bromide (61 mg, $0.4 \mathrm{mmol}, 2$ eq.) was added. The reaction mixture was stirred at RT for 2 hours before the addition of ethanol $(2 \mathrm{~mL})$ and the reaction mixture was concentrated in vacuo. After purification of the crude product with column chromatography $\left(\mathrm{SiO}_{2}, n\right.$-pentane:diethyl ether =9:1), a colorless oil was obtained (37 mg, $91 \%)$.

$\mathbf{R}_{\boldsymbol{f}}=0.11$ (n-pentane:diethyl ether = 9:1); ${ }^{1} \mathbf{H}$ NMR (600 MHz, Chloroform- $d$ ) $\delta 8.60$ (s, 1H; OH), 7.29 - 7.22 (m, 1H; H4), 7.11 (dd, $J=7.7,1.7$ Hz, 1H; H6), 6.92 (dd, $J=8.1,1.2$ Hz, 1H; H3), 6.86 (td, $J=7.5,1.2$ Hz, 1H; H5), 6.14 (s, 1H; H8), 5.95 (ddt, $J=17.2,10.4,5.9$ Hz, 1H; H14), 5.37 - 5.23 (m, 2H; H15), 4.84 (s, 2H; H12), 4.23 (q, J = 7.1 Hz, 2H; H10), 4.20 (dt, $J=5.9,1.3 \mathrm{~Hz}, 2 \mathrm{H}$; H13), 1.32 (t, $J=7.1 \mathrm{~Hz}, 3 \mathrm{H} ; \mathrm{H11}) ;{ }^{13} \mathbf{C}$ NMR (151 MHz, Chloroformd) $\delta 165.5$ (C9), 154.8 (C7), 152.0 (C2), 132.9 (C14), 130.8 (C4), 129.8 (C6), 127.9 (C1), 124.4 (C8), 120.0 (C5), 118.9 (C15), 117.7 (C3), 72.4 (C13), 67.3 (C12), 60.5 (C10), 14.2 (C13); HRESI-MS: m/z $285.1113\left([\mathrm{M}+\mathrm{Na}]^{+}\right.$, calcd. for $\mathrm{C}_{15} \mathrm{H}_{18} \mathrm{O}_{4} \mathrm{Na}^{+}$: 285.1097); IR (ATR): $\tilde{v}=3269$ (br), 2875 (w), 1736 (s), 1623 (m), 1471 (m), 1425 (w), 1378 (m), 1230 (w), 1193 (m), 1110 (w), $1034(\mathrm{w}), 897(\mathrm{~m}), 741(\mathrm{~s}) \mathrm{cm}^{-1}$. 


\section{Ethyl 2-bromohept-6-enoate (S-27)}

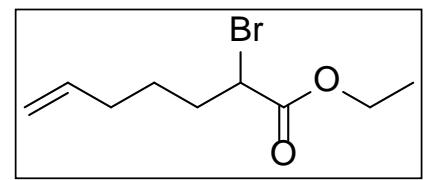

To a solution of diisopropylamine ( $0.46 \mathrm{~g}, 4.5 \mathrm{mmol}, 1.13 \mathrm{eq}$.$) in$ THF (8 mL) cooled to $-78^{\circ} \mathrm{C}, n$-BuLi (2.81 mL, $4.5 \mathrm{mmol}, 1.13 \mathrm{eq}$, in hexanes, $1.6 \mathrm{M}$ ) was added dropwise and mixture was stirred for 1 hour. A solution of ethyl hept-6-enoate $(0.63 \mathrm{~g}, 4 \mathrm{mmol}$, 1eq.) and trimethylsilyl chloride (0.65 g, $6 \mathrm{mmol}, 1.5$ eq.) in THF ( $8 \mathrm{~mL}$ ) was added dropwise to the previously prepared LDA solution and the reaction mixture was stirred for 1 hour. $N$-Bromosuccinimide $(0.80 \mathrm{~g}, 4.5$ mmol, 1.13 eq.) was added and mixture was allowed to warm to RT. The reaction mixture was quenched by saturated aqueous sodium hydrogen carbonate $(10 \mathrm{~mL})$ and diluted with water (50 $\mathrm{mL})$. Organics were extracted with $\mathrm{CH}_{2} \mathrm{Cl}_{2}(2$ x $20 \mathrm{~mL})$, dried over $\mathrm{MgSO}_{4}$ and concentrated in vacuo. After purification of the crude product with column chromatography $\left(\mathrm{SiO}_{2}, n\right.$ pentane:diethyl ether $=98: 2)$, a colorless oil was obtained $(0.72 \mathrm{~g}, 77 \%)$.

$\mathbf{R}_{\boldsymbol{f}}=0.60$ (cyclohexane:ethyl acetate $=9: 1$ ); ${ }^{1} \mathbf{H}$ NMR (400 MHz, Chloroform- $d$ ) $\delta 5.78$ (ddt, $J=16.9,10.1,6.6 \mathrm{~Hz}, 1 \mathrm{H}), 5.10-4.91$ (m, 2H), $4.34-4.09$ (m, 3H), $2.18-1.91$ (m, 4H), $1.60-1.43$ (m, 2H), 1.30 (t, $J=7.1 \mathrm{~Hz}, 3 \mathrm{H}) .{ }^{13} \mathrm{C}$ NMR (101 MHz, Chloroform-d) $\delta$ 169.8, $137.7,115.4,62.0,46.0,34.3,32.9,26.5,14.0$.

Data in agreement with literature. ${ }^{[4]}$

\section{Ethyl (E)-2-(2-(methoxymethoxy)benzylidene)hept-6-enoate (S-28)}

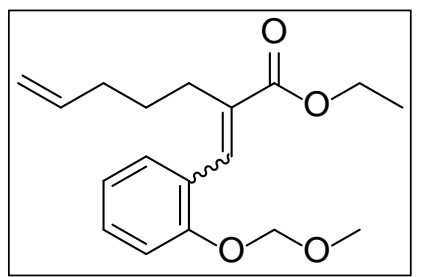

Ester S-27 (0.58 g, 2.5 mmol, 1 eq.) was mixed with triethyl phosphite (0.83 g, $5 \mathrm{mmol}$, 2 eq.) and heated to $160{ }^{\circ} \mathrm{C}$ for 5 hours. The reaction mixture was then cooled and filtered through a plug of silica gel with $\mathrm{CH}_{2} \mathrm{Cl}_{2}$ as an eluent. Volatiles were then evaporated ( $25^{\circ} \mathrm{C}, 0.1$ torr). The crude residue (phosphonate intermediate) was then added to an oven dried Schlenk tube containing sodium hydride ( $80 \mathrm{mg}, 2 \mathrm{mmol}, 0.8 \mathrm{eq.,} 60 \%$ in paraffin oil) in THF (5 mL) under an argon atmosphere. The reaction mixture was stirred for 15 minutes before the addition of aldehyde S-1 (0.33g, 2 mmol, 0.8 eq.). The reaction mixture was stirred for 20 hours at RT and was quenched with saturated ammonium chloride solution (5 ml) and diluted with water $(25 \mathrm{~mL})$. Organic were extracted with $\mathrm{CH}_{2} \mathrm{Cl}_{2}(2 \times 30 \mathrm{~mL})$ dried over $\mathrm{MgSO}_{4}$ and concentrated in vacuo. After purification of the crude product with column chromatography $\left(\mathrm{SiO}_{2}, n\right.$-pentane:diethyl ether $\left.=9: 1\right)$, an inseparable mixture of $E$ and $Z$ isomers was obtained (0.18 g, $E=77 \%, Z=23 \%)$. 
Ethyl (E)-2-(2-hydroxybenzylidene)hept-6-enoate (S-29)

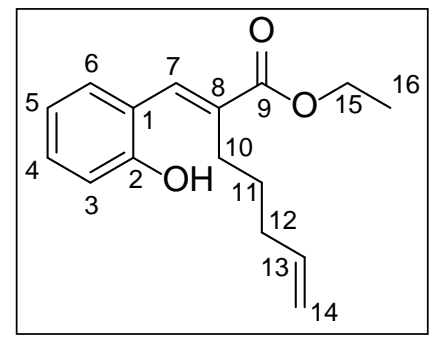

Mixture of isomers S-28 (E = 77\%, $\mathrm{Z}=23 \%$; $0.18 \mathrm{~g}, 0.60 \mathrm{mmol}, 1$ eq.) was dissolved in dry $\mathrm{CH}_{2} \mathrm{Cl}_{2}(1.5 \mathrm{~mL})$ before the dropwise addition of trimethyl silyl bromide (183 mg, $1.2 \mathrm{mmol}, 2$ eq.). The reaction mixture was stirred at RT for 2 hours before the addition of ethanol (2 mL) and the reaction mixture was concentrated in vacuo. After purification of the crude product with column chromatography $\left(\mathrm{SiO}_{2}, n\right.$-pentane:diethyl ether =9:1), a colorless oil was obtained (37 mg, 61\% - calculated for pure E isomer).

$\mathbf{R}_{\boldsymbol{f}}=0.35$ (n-pentane:diethyl ether = 9:1); ${ }^{1} \mathbf{H}$ NMR (600 MHz, Chloroform- $d$ ) $\delta 7.68$ (s, 1H; H7), 7.23 (tdd, $J=8.0,1.7,0.6 \mathrm{~Hz}, 1 \mathrm{H} ; \mathrm{H} 4), 7.17$ (dt, $J=7.7,1.1 \mathrm{~Hz}, 1 \mathrm{H} ; \mathrm{H6}$ ), 6.96 - 6.92 (m, 1H; H5), 6.88 (dd, $J=8.1,1.1 \mathrm{~Hz}, 1 \mathrm{H}$; H3), 5.75 (ddt, $J=16.9,10.2,6.6 \mathrm{~Hz}, 1 \mathrm{H} ; \mathrm{H} 13$ ), 5.10 (b, 1H; OH), 4.99 - 4.87 (m, 2H; H14), 4.28 (q, $J=7.1$ Hz, 2H; H15), 2.49 - 2.39 (m, 2H; H10), 2.08 - 2.00 (m, 2H; H12), 1.62 - 1.55 (m, 2H; H11), 1.35 (t, J = 7.1 Hz, 3H; H16); ${ }^{13} \mathrm{C}$ NMR (151 MHz, Chloroform-d) $\delta 168.0$ (C9), 153.2 (C2), 138.2 (C13), 136.2 (C8), 133.5 (C7), 129.8 (C4), 129.4 (C6), 122.7 (C1), 120.6 (C5), 115.7 (C3), 114.7 (C14), 60.9 (C15), 33.6 (C11), 28.4 (C10), 27.4 (C12), 14.3 (C16); HR-ESI-MS: m/z 283.1316 ([M+Na] ${ }^{+}$, calcd. for $\mathrm{C}_{16} \mathrm{H}_{20} \mathrm{O}_{3} \mathrm{Na}^{+}$: 283.1310); IR (ATR): ṽ = 3419 (br), 2958 (w), 2868 (w), 1677 (s), 1603 (m), 1454 (s), 1368 (m), 1298 (m), 1191 (m), 1156 (m), 991 (w), 780 (s) cm².

\section{4-(Pent-4-en-1-yl)-2H-chromen-2-one (12)}

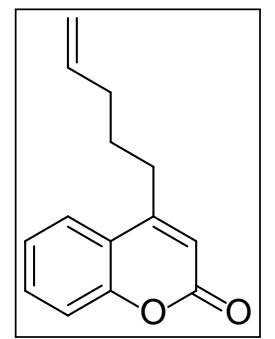

Prepared according to a literature procedure. ${ }^{[6]}$ In an oven dried and argon flushed Schlenk tube a Grignard reagent was prepared by adding 5-bromopent1-ene (0.53g, $3 \mathrm{mmol}, 1.5$ eq.) to iodine-activated magnesium turnings $(0.20$ g, 8 mmol, 4 eq.) in dry THF (10 mL). In a separate dry and argon flushed Schlenk tube a solution consisting of anhydrous iron(II) chloride (25 mg, 0.2 mmol), hexamethylphosphoramide (0.72 g, 4 mmol, 2 eq.) and 2-oxo-2H-chromen-4-yl acetate ( $0.41 \mathrm{~g}, 2 \mathrm{mmol}, 1$ eq.) in THF $(10 \mathrm{~mL})$ was prepared and chilled to $0{ }^{\circ} \mathrm{C}$. The Grignard solution was transferred dropwise to the second mixture via syringe. The reaction was stirred for 2 hours and was quenched with saturated solution of ammonium chloride $(5 \mathrm{~mL})$ and diluted with water (25 mL). Organics were extracted with $\mathrm{CH}_{2} \mathrm{Cl}_{2}\left(2\right.$ x $30 \mathrm{~mL}$ ), dried over $\mathrm{MgSO}_{4}$ and concentrated in vacuo. After purification of the crude product with column chromatography $\left(\mathrm{SiO}_{2}\right.$, cyclohexane:ethyl acetate $=7: 3)$, a colorless oil was obtained $(0.29 \mathrm{~g}, 68 \%)$. 
$\mathbf{R}_{\boldsymbol{f}}=0.60$ (cyclohexane:ethyl acetate = 7:3); ${ }^{1} \mathbf{H}$ NMR $(600 \mathrm{MHz}$, Chloroform- $d) \delta 7.62$ (dd, $J=8.0,1.6 \mathrm{~Hz}, 1 \mathrm{H}$ ), 7.52 (ddd, $J=8.6,7.2,1.5 \mathrm{~Hz}, 1 \mathrm{H}$ ), 7.34 (dd, $J=8.3,1.2 \mathrm{~Hz}, 1 \mathrm{H}$ ), 7.29 (ddd, $J=8.4,7.3,1.2 \mathrm{~Hz}, 1 \mathrm{H}$ ), 6.29 (d, $J=1.1 \mathrm{~Hz}, 1 \mathrm{H}$ ), 5.83 (ddt, $J=17.0,10.2,6.7 \mathrm{~Hz}$, 1H), 5.12 - 5.02 (m, 2H), $2.81-2.75$ (m, 2H), 2.21 (qt, $J=7.2,1.4$ Hz, 2H), $1.85-1.77$ (m, 2H). ${ }^{13}$ C NMR (151 MHz, Chloroform-d) $\delta$ 161.1, 156.1, 153.9, 137.5, 131.8, 124.4, 124.3, 119.4, 117.5, 116.0, 114.2, 77.4, 77.0, 33.4, 31.1, 27.3.

Data in agreement with literature. ${ }^{[5]}$

\section{2-Oxo-2H-chromen-4-yl acetate (S-30)}

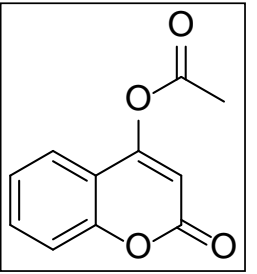

To a solution of 2-hydroxychromen-4-one (4.05 g, $25 \mathrm{mmol}, 1$ eq.) in $\mathrm{CH}_{2} \mathrm{Cl}_{2}$ (50 mL), acetic anhydride (3.06 g, $30 \mathrm{mmol}, 1.2$ eq.) was added, followed by triethylamine ( $3.54 \mathrm{~g}, 35 \mathrm{mmol}, 1.4 \mathrm{eq}$.). The reaction mixture was heated to reflux for 3 hours. After cooling to RT, the organic phase was washed with water $(50 \mathrm{~mL})$ and saturated sodium bicarbonate solution $(50 \mathrm{~mL})$. Organics were dried over $\mathrm{MgSO}_{4}$ and concentrated in vacuo. The crude material was recrystallized from hot toluene to give the product as white needles (2.26 g, $44 \%)$.

$\mathrm{R}_{f}=0.50$ (cyclohexane:ethyl acetate $=7: 3$ ); ${ }^{1} \mathrm{H}$ NMR (400 MHz, Chloroform-d) $\delta 7.63$ (dd, J = 7.9, 1.6 Hz, 1H), 7.59 (ddd, J = 8.8, 7.4, 1.6 Hz, 1H), 7.37 (dd, J = 8.3, 1.1 Hz, 1H), 7.34 - 7.28 (m, 1H), 6.52 (s, 1H), 2.45 (s, 3H); ${ }^{13} \mathrm{C}$ NMR (101 MHz, Chloroform-d) $\delta 166.71$, 161.58, 158.41, 153.80, 132.93, 124.46, 122.83, 117.25, 115.54, 105.39, 21.41. Data in agreement with literature. ${ }^{[6]}$

\section{Ethyl (E)-3-(2-hydroxyphenyl)but-2-enoate (10)}

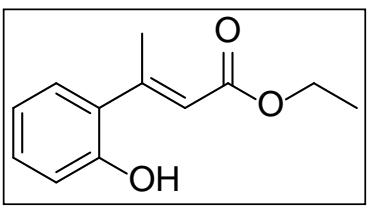

A solution of 2'-hydroxyacetophenone (1.14g, $8.4 \mathrm{mmol}, 1$ eq.) and ethyl (triphenylphosphoranylidene)acetate (5.05 g, $14.5 \mathrm{mmol}, 1.73$ eq.) in toluene $(20 \mathrm{~mL})$ was stirred at $100{ }^{\circ} \mathrm{C}$ for 4 hours. After completion, the reaction mixture was allowed to cool to RT and was concentrated in vacuo. After purification of the crude product with column chromatography $\left(\mathrm{SiO}_{2}, n\right.$-pentane:diethyl ether $=7: 3)$, a white solid was obtained $(0.55 \mathrm{~g}, 34 \%)$.

$\mathbf{R}_{\boldsymbol{f}}=0.60$ (n-pentane:diethyl ether = 7:3); ${ }^{1} \mathbf{H}$ NMR $(600 \mathrm{MHz}$, Chloroform- $d) \delta 7.21$ (ddd, $J=8.1,7.4,1.7 \mathrm{~Hz}, 1 \mathrm{H}), 7.17-7.12$ (m, 1H), 6.93 (td, $J=7.5,1.1 \mathrm{~Hz}, 1 \mathrm{H}), 6.89$ (ddd, $J=$ 
8.1, 1.2, $0.4 \mathrm{~Hz}, 1 \mathrm{H}), 6.00$ (q, $J=1.4 \mathrm{~Hz}, 1 \mathrm{H}), 5.27$ (s, 1H), 4.22 (q, $J=7.1 \mathrm{~Hz}, 2 \mathrm{H}), 2.53$ (d, $J=1.4 \mathrm{~Hz}, 3 \mathrm{H}), 1.31$ (t, $J=7.1 \mathrm{~Hz}, 3 \mathrm{H}) .{ }^{13} \mathrm{C}$ NMR (151 MHz, Chloroform- $d$ ) $\delta$ 166.6, 154.3, 151.9, 130.1, 129.8, 128.5, 120.9, 120.1, 116.3, 60.2, 20.3, 14.4.

UV-Vis of $10 ; \mathrm{c}=1 \mathrm{e}-4 \mathrm{~mol} / \mathrm{l}$

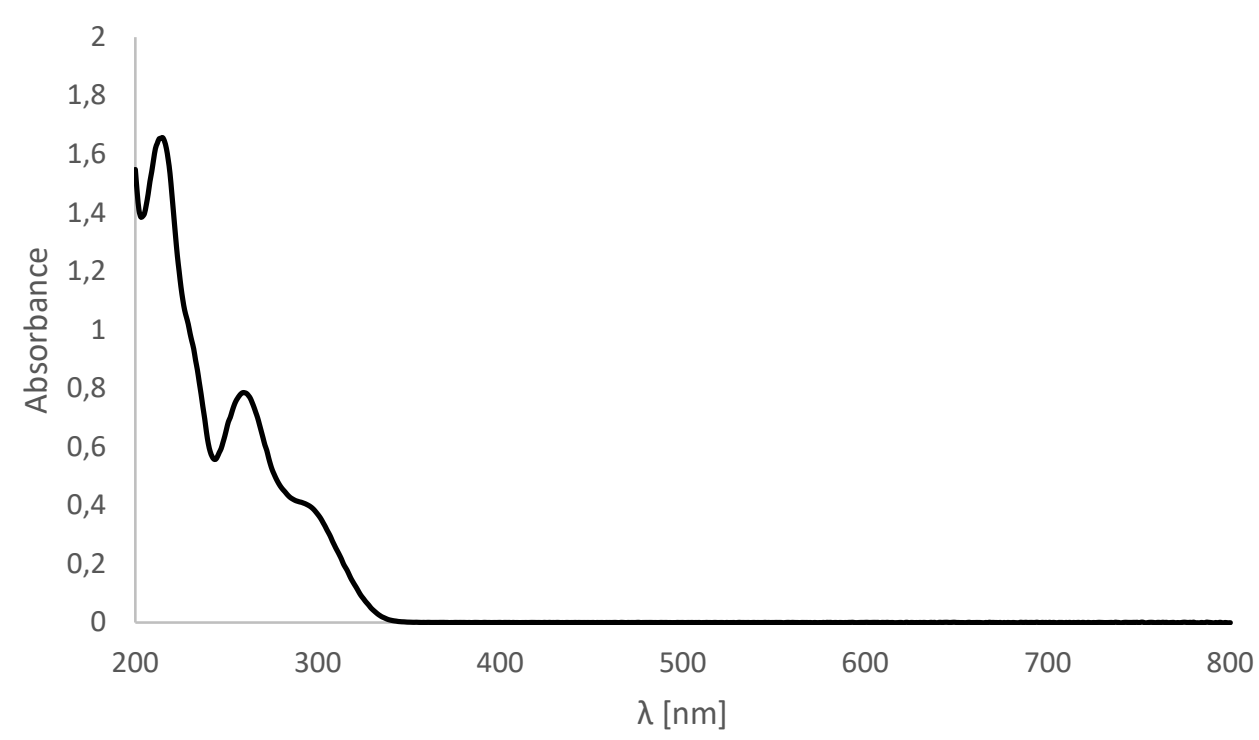

Data in agreement with literature. ${ }^{[7]}$

\section{Sequential Energy Transfer Protocol - Substrate Scope}

\section{General Procedure A}

The specified phenolic precursor (0.1 mmol, 1eq.) and thioxanthone $(0.005 \mathrm{mmol}, 0.05$ eq.) were dissolved in acetonitrile $(2 \mathrm{~mL})$. The reaction mixture was degassed by purging with argon for 5 minutes and the vessel was irradiated at $402 \mathrm{~nm}$ for 24 hours at $50{ }^{\circ} \mathrm{C}$ (a consequence of the irradiation apparatus). After the reaction was complete solvent was removed under reduced pressure and the mixture was purified via column chromatography ( $\mathrm{SiO} 2, \mathrm{SiO}_{2}$ - specified combination of solvents). 


\section{Dihydrocoumarin (2)}

Prepared according to General Procedure A. After purification of the crude product with column chromatography ( $\mathrm{SiO}_{2}, n$-pentane:diethyl ether =9:1), a colorless oil was obtained (17.1 mg, 80\%).

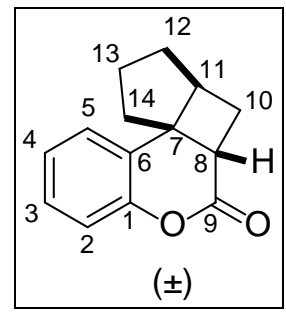

$\mathbf{R}_{\boldsymbol{f}}=0.20$ (n-pentane:diethyl ether $\left.=8: 2\right) ;{ }^{1} \mathbf{H}$ NMR (600 MHz, Chloroform- $d$ ) $\delta 7.22$ (ddd, $J=8.1,7.0,1.9 \mathrm{~Hz}, 1 \mathrm{H}$; H3), 7.17 (dd, $J=7.8,1.9 \mathrm{~Hz}, 1 \mathrm{H} ; \mathrm{H} 5$ ), 7.13 (ddd, $J=7.8,7.0,1.3$ Hz, 1H; H4), 7.02 (dd, $J=8.1,1.2$ Hz, 1H; H2), 3.17 (ddd, $J=10.9,7.8,1.0 \mathrm{~Hz}, 1 \mathrm{H} ; \mathrm{H} 8$ ), 2.64 (dddt, $J$ = 9.5, 7.3, 3.2, $1.1 \mathrm{~Hz}, 1 \mathrm{H} ; \mathrm{H} 11$ ), 2.59 (dddd, $J=12.6,9.5,7.8,0.9 \mathrm{~Hz}, 1 \mathrm{H} ; \mathrm{H} 10$ ), 2.19 - 2.07 (m, 3H; H14, H10', H12), 2.01 - 1.84 (m, 3H, H12', H13, H14'), 1.72 (dddd, J = 14.2, 4.8, 3.2, $1.1 \mathrm{~Hz}, 1 \mathrm{H}$; H13’); ${ }^{13} \mathrm{C}$ NMR (151 MHz, Chloroform-d) $\delta 169.3$ (C9), 150.8 (C1), 128.4 (C3), 126.30 (C6), 126.20 (C5), 125.2 (C4), 117.6 (C2), 48.8 (C7), 47.4 (C11), 41.0 (C14), 38.0 (C8), 33.4 (C13), 28.8 (C10), 26.1 (C12); HR-ESI-MS: m/z 237.0884 ([M+Na]+, calcd. for $\mathrm{C}_{14} \mathrm{H}_{14} \mathrm{NaO}_{2}{ }^{+}$: 237.0886); IR (ATR): $\tilde{v}=2941$ (w), 2857 (w), 1754 (s), 1654 (w), 1584 (w), 1490 (m), 1447 (m), 1335 (w), 1196 (s), 1083 (m), 966 (m), 754 (s) cm-1.

Data in agreement with literature. ${ }^{[8]}$

NOESY spectra of key contacts are shown on page 35.

\section{Sequential Energy Transfer Protocol - Larger scale synthesis of 2}

The precursor 1 ( $1 \mathrm{mmol}$, 1eq.) and thioxanthone (0.05 mmol, 0.05 eq.) were dissolved in acetonitrile $(20 \mathrm{~mL})$. The reaction mixture was degassed by purging with argon for 5 minutes and the vessel was irradiated at $402 \mathrm{~nm}$ for 72 hours at $50{ }^{\circ} \mathrm{C}$ (a consequence of the irradiation apparatus). After the reaction was complete solvent was removed under reduced pressure and the mixture was purified via column chromatography ( $\mathrm{SiO}_{2}, n$-pentane:diethyl ether =9:1), a colorless oil was obtained (152 mg, 71\%). 


\section{Dihydrocoumarin (3)}

Prepared according to General Procedure A. After purification of the crude product with column chromatography ( $\mathrm{SiO}_{2}, n$-pentane:diethyl ether =9:1), a colorless oil was obtained (17.2 mg, $61 \%)$.

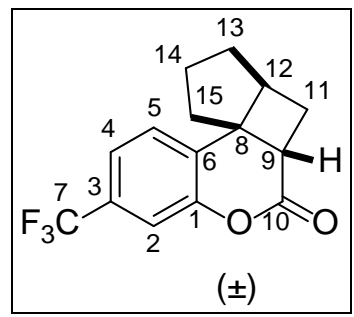

$\mathbf{R}_{\boldsymbol{f}}=0.60$ (n-pentane:diethyl ether = 7:3); ${ }^{1} \mathbf{H}$ NMR $(600 \mathrm{MHz}$, Chloroform- $d$ ) $\delta 7.38$ (ddt, $J=8.1,1.8,0.7 \mathrm{~Hz}, 1 \mathrm{H}$; H4), 7.30 - 7.26 (m, 2H; H2, H5), 3.21 (ddd, $J=11.2,8.0,1.1 \mathrm{~Hz}$, 1H; H9), 2.71 - 2.64 (m, 1H; H12), 2.60 (dddd, $J=12.8,9.6,8.0,0.7$ Hz, 1H; H11), 2.20 2.07 (m, 3H; H11', H13), 2.02 - 1.97 (m, 1H; H15), 1.97 - 1.89 (m, 2H; H15', H14), 1.79 1.72 (m, 1H; H14'); ${ }^{13}$ C NMR (151 MHz, Chloroform-d) $\delta 168.1$ (C10), 150.9 (C1), 130.9 (q, $J=33.2 \mathrm{~Hz}$; C3), 130.5 (C6), 127.0 (C5), 123.6 (q, $J=272.1 \mathrm{~Hz}$; C7), 121.8 (q, $J=3.7 \mathrm{~Hz}$; C4), 115.0 (q, $J=4.0 \mathrm{~Hz}$; C2)), 48.8 (C8), 47.8 (C12), 41.0 (C15), 37.7 (C9), 33.4 (C14), 28.8 (C11), 26.1 (C12); ${ }^{19}$ F NMR (564 MHz, Chloroform- $d$ ) $\delta$-62.83; HR-ESI-MS: m/z 337.1034 $\left(\left[\mathrm{M}+\mathrm{Na}+\mathrm{CH}_{3} \mathrm{OH}\right]^{+}\right.$, calcd. for $\left(\mathrm{C}_{15} \mathrm{H}_{13} \mathrm{O}_{2} \mathrm{~F}_{3} \mathrm{NaCH}_{3} \mathrm{OH}^{+}\right)$: 337.1027); HR-ESI-MS: $\mathrm{m} / \mathrm{z}$ $297.1478\left([\mathrm{M}+\mathrm{Na}]^{+}\right.$, calcd. for $\mathrm{C}_{17} \mathrm{H}_{22} \mathrm{O}_{3} \mathrm{Na}^{+}$: 297.1467). IR (ATR): $\tilde{v}=2940(\mathrm{w}), 2931(\mathrm{w})$ 2864 (w), 1764 (s), 1639 (w), 1500 (w), 1480 (w), 1368 (m), 1298 (m), 1191(m), 899 (w), 870 (w) $\mathrm{cm}^{-1}$.

\section{Dihydrocoumarin (4)}

Prepared according to General Procedure A. After purification of the crude product with column chromatography ( $\mathrm{SiO}_{2}, n$-pentane:diethyl ether =9:1), a colorless oil was obtained (20.3 mg, 94\%).

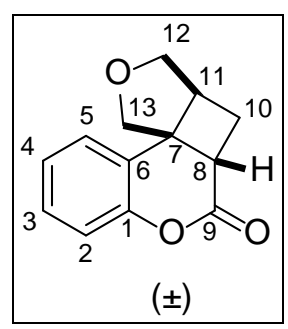


$\mathbf{R}_{\boldsymbol{f}}=0.34$ (cyclohexane:ethyl acetate = 7:3); ${ }^{1} \mathbf{H}$ NMR (600 MHz, Chloroform- $d$ ) $\delta 7.28$ (ddd, $J=8.2,7.2,1.7 \mathrm{~Hz}, 1 \mathrm{H}$; H3), 7.22 (dd, $J=7.8,1.7 \mathrm{~Hz}, 1 \mathrm{H} ; \mathrm{H} 5$ ), 7.16 (ddd, $J$ = 7.8, 7.2, $1.3 \mathrm{~Hz}, 1 \mathrm{H} ; \mathrm{H} 4), 7.06$ (dd, $J$ = 8.2, $1.3 \mathrm{~Hz}, 1 \mathrm{H} ; \mathrm{H} 2$ ), 4.09 - 4.02 (m, 2H; H12a, H13a), 3.98 3.92 (m, 1H; H12b), 3.77 (d, $J=9.7$ Hz, 1H; H13b), 3.47 (ddd, $J=11.0,8.1,1.3$ Hz, 1H; H8), $2.91-2.85$ (m, 1H; H11), 2.62 (dddd, $J=12.8,9.0,8.1,0.8 \mathrm{~Hz}, 1 \mathrm{H} ; \mathrm{H} 10 \mathrm{~b}), 2.36$ (ddd, $J=$ 12.8, 11.0, 3.9 Hz, 1H; H10a); ${ }^{13}$ C NMR (151 MHz, Chloroform-d) $\delta 168.2$ (C9), 151.5 (C1), 129.3 (C3), 126.1 (C5), 125.3 (C4), 121.1 (C6), 118.0 (C2), 78.5 (C13), 75.1 (C12), 49.7 (C7),

48.3 (C11), 37.5 (C8), 28.5 (C10); HR-ESI-MS: m/z 239.0681 ([M+Na] $]^{+}$, calcd. for $\mathrm{C}_{13} \mathrm{H}_{12} \mathrm{O}_{3} \mathrm{Na}^{+}$: 239.0679); IR (ATR): $\tilde{v}=2340$ (w), 2855 (w), 1756 (s), 1721 (w), 1492 (m), 1450 (m), 1420 (w), 1258 (m), 1196 (s), 1080 (m), 966 (m), 754 (s) cm².

Data in agreement with literature. ${ }^{[8]}$

\section{Dihydrocoumarin (5)}

Prepared according to General Procedure A. After purification of the crude product with column chromatography ( $\mathrm{SiO}_{2}$, $n$-pentane:diethyl ether =9:1), a colorless oil was obtained (25.2 mg, 83\%).

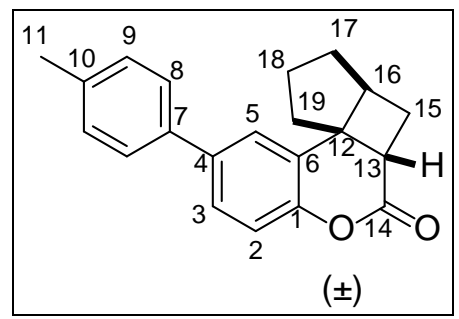

$\mathbf{R}_{\boldsymbol{f}}=0.60$ (n-pentane:diethyl ether = 7:3); ${ }^{1} \mathbf{H}$ NMR $(600 \mathrm{MHz}$, Chloroform- $d$ ) $\delta 7.44-$ 7.40 (m, 3H; H3, H8), 7.32 (d, $J=2.2$ Hz, 1H; H5), 7.25 (d, $J=7.7$ Hz, 2H; H9), 7.08 (d, $J=$ 8.5 Hz, 1H; H2), 3.20 (ddd, $J$ = 11.1, 8.1, 1.1 Hz, 1H; H13), 2.74 - 2.67 (m, 1H; H16), 2.66 2.58 (m, 1H; H15), 2.40 (s, 3H; H11), 2.19 - 2.10 (m, 3H; H; H15', H17, H19), 2.05 - 1.97 (m, 2H; H17’; H19’), 1.97 - 1.89 (m, 1H; H18), 1.74 (dt, $J$ = 13.7, 3.9 Hz, 1H; H18'); ${ }^{13}$ C NMR (151 MHz, Chloroform-d) $\delta 169.3$ (C14), 150.1 (C1), 138.5 (C4), 137.6 (C7), 137.4 (C10), 129.7 (C9), 127.01 (C3), 126.93 (C8), 126.5 (C6), 124.7 (C5), 117.9 (C2), 49.0 (C12), 47.5 (C16), 41.1 (C19), 37.9 (C13), 33.4 (C18), 28.8 (C15), 26.1 (C17), 21.2 (C11); HR-ESI-MS: $\mathrm{m} / \mathrm{z} 359.1615\left(\left[\mathrm{M}+\mathrm{Na}+\mathrm{CH}_{3} \mathrm{OH}\right]^{+}\right.$, calcd. for $\left(\mathrm{C}_{21} \mathrm{H}_{20} \mathrm{O}_{2} \mathrm{NaCH}_{3} \mathrm{OH}\right)^{+}: 359.1618$ IR (ATR): $\tilde{v}=$ 2960 (w), 2854 (w), 1714 (s), 1643 (w), 1575 (w), 1457 (m), 1422 (m), 1203 (w), 1170 (s), $1071(\mathrm{~m}), 923(\mathrm{~m}), 812(\mathrm{~s}) \mathrm{cm}^{-1}$. 


\section{Dihydrocoumarin (6)}

Prepared according to General Procedure A. After purification of the crude product with column chromatography ( $\mathrm{SiO}_{2}, n$-pentane:diethyl ether =9:1), a colorless oil was obtained (25.5 mg, $86 \%)$.

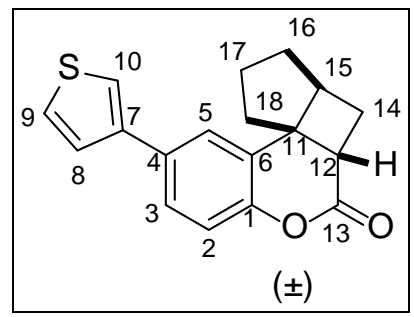

$\mathbf{R}_{\boldsymbol{f}}=0.40$ (n-pentane:diethyl ether = 7:3); ${ }^{1} \mathbf{H}$ NMR (400 MHz, Chloroform- $d$ ) $\delta 7.44$ (dd, $J=8.4,2.2 \mathrm{~Hz}, 1 \mathrm{H}$; H3), 7.39 (d, $J=3.2 \mathrm{~Hz}, 2 \mathrm{H}$; H9, H10), 7.36 - 7.30 (m, 2H; H5, H8), 7.06 (d, $J=8.4$ Hz, 1H; H2), 3.25 - 3.10 (m, 1H; H12), 2.73 - 2.66 (m, 1H; H15), 2.66 - 2.55 (m, 1H; H14), 2.21 - 2.08 (m, 3H; H14', H16, H18), 2.01 - 1.97 (m, 2H; H16’, H18'), 1.97 - 1.88 (m, 1H; H17’), 1.74 (dt, $\left.J=13.6,4.3 \mathrm{~Hz}, 1 \mathrm{H} ; \mathrm{H} 17^{\prime}\right) ;{ }^{13} \mathrm{C}$ NMR (126 MHz, Chloroform-d) $\delta$ 169.1, 150.1, 141.6, 133.3, 126.61, 126.61, 126.59, 126.4, 124.1, 120.3, 118.0, 48.9, 47.4, 41.1, 37.9, 33.4, 28.8, 26.1; HR-ESI-MS: $\mathrm{m} / \mathrm{z} 351.1026\left(\left[\mathrm{M}+\mathrm{Na}+\mathrm{CH}_{3} \mathrm{OH}\right]^{+}\right.$, calcd. for $\left(\mathrm{C}_{18} \mathrm{H}_{16} \mathrm{O}_{2} \mathrm{SNaCH}_{3} \mathrm{OH}\right)^{+}$: 351.1025); IR (ATR): $\tilde{v}=2951$ (w), 2867 (w), 1735 (s), $1624(\mathrm{w})$, 1514 (w), 1430 (m), 1337 (w), 1315 (w), 1120 (s), 1083 (m), 956 (m), 799 (s) cm-1.

\section{Dihydrocoumarin (7)}

Prepared according to General Procedure A. After purification of the crude product with column chromatography ( $\mathrm{SiO}_{2}, n$-pentane:diethyl ether =9:1), a colorless oil was obtained (21.4 mg, 73\%). The oil spontaneously crystalized after several hours.

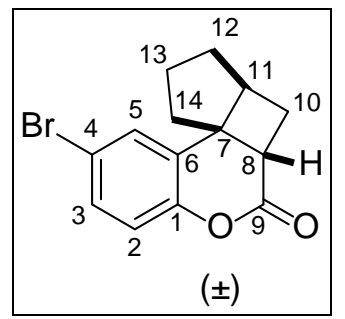

$\mathbf{R}_{\boldsymbol{f}}=0.20$ (n-pentane:diethyl ether = 8:2); ${ }^{1} \mathbf{H}$ NMR (500 MHz, Chloroform- $d$ ) $\delta 7.32$ (dd, $J=8.7,2.4 \mathrm{~Hz}, 1 \mathrm{H}$; H3), 7.26 (d, $J=2.4 \mathrm{~Hz}, 1 \mathrm{H} ; \mathrm{H} 5), 6.91$ (d, $J=8.7 \mathrm{~Hz}, 1 \mathrm{H} ; \mathrm{H} 2), 3.16$ (ddd, $J=11.1,8.0,1.1 \mathrm{~Hz}, 1 \mathrm{H}$; H8), $2.70-2.63$ (m, 1H; H11), 2.58 (dddd, $J=12.8$, 9.6, 8.0, 0.7 Hz, 1H; H10), 2.18 - 2.06 (m, 3H; H10’, H12), 2.00 - 1.86 (m, 3H; H13, H14), 1.77 - 1.68 (m, 1H; H13’); ${ }^{13}$ C NMR (126 MHz, Chloroform-d) $\delta$ 168.4, 149.8, 131.2, 129.0, 128.4, 119.2, 
117.5, 48.6, 47.4, 40.8, 37.4, 33.2, 28.6, 25.9; HR-ESI-MS: m/z 347.0264 ([M+Na+CH $3 \mathrm{OH}]^{+}$, calcd. for $\left(\mathrm{C}_{14} \mathrm{H}_{13} \mathrm{O}_{2} \mathrm{BrNaCH}_{3} \mathrm{OH}\right)^{+}$: 347.0253); IR (ATR): $\tilde{v}=2933(\mathrm{w}), 2859(\mathrm{w}), 2820(\mathrm{w})$, 1760 (s), 1636 (w), 1484 (w), 1390 (m), 1347 (m), 1235 (w), 1178 (s), 1052 (m), 872 (m), 744 (w) $\mathrm{cm}^{-1}$. Melting point: $120-123^{\circ} \mathrm{C}$ (pentane - acetone).

\section{Dihydrocoumarin (8)}

Prepared according to General Procedure A. After purification of the crude product with column chromatography ( $\mathrm{SiO}_{2}, n$-pentane:diethyl ether $\left.=9: 1\right)$, a colorless oil was obtained (18.0 mg, 84\%).

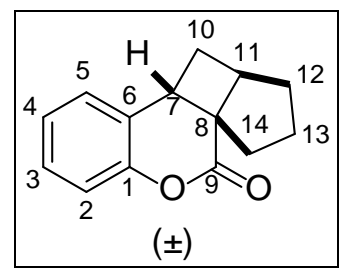

$\mathbf{R}_{\boldsymbol{f}}=0.60$ (n-pentane:diethyl ether = 9:1); ${ }^{1} \mathbf{H}$ NMR (600 MHz, Chloroform- $d$ ) $\delta 7.21$ (ddd, $J=8.3,7.4,1.7 \mathrm{~Hz}, 1 \mathrm{H}$; H3), 7.08 (td, $J=7.4,1.2 \mathrm{~Hz}, 1 \mathrm{H} ; \mathrm{H} 4), 7.04-6.99$ (m, 2H; H1, H5), 3.29 (dd, $J=9.7,6.2 \mathrm{~Hz}, 1 \mathrm{H}$; H7), 3.25 - 3.18 (m, 1H; H11), 2.38 (ddd, $J=12.6,11.6,7.5 \mathrm{~Hz}$, 1H; H14), 2.20 - 2.07 (m, 4H; H10, H12), 1.95 (ddt, $J=12.7,5.8,1.3$ Hz, 1H; H14'), 1.84 (ddt, $J=13.0,11.3,7.4 \mathrm{~Hz}, 1 \mathrm{H} ; \mathrm{H} 13$ ), 1.66 (ddd, $J=13.7,5.9,1.4 \mathrm{~Hz}, 1 \mathrm{H} ; \mathrm{H} 13$ '); ${ }^{13} \mathbf{C}$ NMR (151 MHz, Chloroform-d) $\delta 172.2$ (C9), 150.4 (C1), 128.2 (C3), 127.9 (C5), 125.09 (C6), 124.96 (C4), 117.4 (C2), 49.5 (C8), 46.5 (C11), 37.8 (C14), 36.4 (C7), 33.4 (C13), 33.1 (C10**), $26.1\left(\mathrm{C} 12^{* *}\right)$; HR-ESI-MS: m/z $237.0889\left([\mathrm{M}+\mathrm{Na}]^{+}\right.$, calcd. for $\mathrm{C}_{14} \mathrm{H}_{14} \mathrm{O}_{2} \mathrm{Na}^{+}$: 237.0886); IR (ATR): $\tilde{v}=2979$ (w), 2841 (w), 1746 (s), 1554 (w), 1500 (w), 1455 (m), 1262 (w), 1196 (m), $1130(\mathrm{~m}), 902(\mathrm{~m}), 754(\mathrm{~s}) \mathrm{cm}^{-1}$.

Data in agreement with literature. ${ }^{[8]}$ 


\section{Dihydrocoumarin (9)}

Prepared according to General Procedure A. After purification of the crude product with column chromatography ( $\mathrm{SiO}_{2}, n$-pentane:diethyl ether =9:1), a colorless oil was obtained (17.0 mg, $75 \%)$.

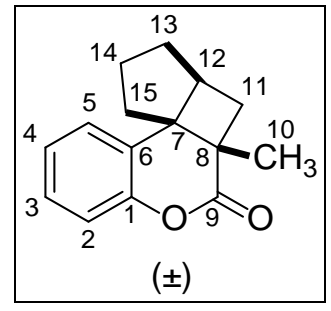

$\mathbf{R}_{\boldsymbol{f}}=0.21$ (n-pentane:diethyl ether = 10:1); ${ }^{1} \mathbf{H}$ NMR (500 MHz, Chloroform- $d$ ) $\delta 7.20$ (ddd, $J$ = 8.0, 7.2, $1.7 \mathrm{~Hz}, 1 \mathrm{H}$; H3), 7.17 (dd, $J=7.9,1.7 \mathrm{~Hz}, 1 \mathrm{H}$; H5), 7.09 (ddd, $J=7.8,7.2$, $1.3 \mathrm{~Hz}, 1 \mathrm{H}$; H4), 6.99 (dd, $J=8.1,1.3 \mathrm{~Hz}, 1 \mathrm{H}$; H2), 2.78 - 2.67 (m, 2H; H12; H11), 2.25 2.16 (m, 1H; H15), 2.14 - 1.96 (m, 3H;H13, H15'), 1.85 - 1.77 (m, 1H; H11'), 1.77 - 1.69 (m, 1H; H14), 1.61 - 1.55 (m, 1H; H14'), 1.47 (s, 3H; H10); ${ }^{13}$ C NMR (126 MHz, Chloroform-d)

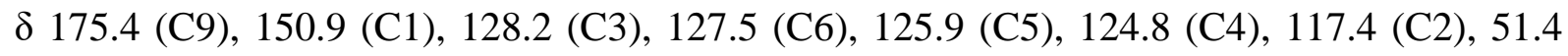
(C7), 46.1 (C12), 41.8 (C8), 37.7 (C11), 34.2 (C15), 32.1 (C14), 27.0 (C13), 20.9 (C10); HRESI-MS: m/z $251.1063\left([\mathrm{M}+\mathrm{Na}]^{+}\right.$, calcd. for $\mathrm{C}_{15} \mathrm{H}_{16} \mathrm{O}_{2} \mathrm{Na}^{+}$: 251.1043); IR (ATR): $\tilde{v}=2963$ (w), 2910 (w), 1738 (s), 1493 (m), 1471 (w), 1330 (w), 1243 (s), 1148 (m), 1001 (m), 943 (m), $754(\mathrm{~s}) \mathrm{cm}^{-1}$.

\section{Probing the reaction mechanism}

\section{General Procedure B - NMR timecourse}

The compound 1 (0.05 mmol, 1eq.) and thioxanthone (0.0025 mmol, 0.05 eq.) were dissolved in deuterated acetonitrile $(1 \mathrm{~mL})$. The reaction mixture was degassed by purging with argon for 5 minutes and the vessel was irradiated at $402 \mathrm{~nm}$ for 24 hours at $50{ }^{\circ} \mathrm{C}$ (a consequence of the irradiation apparatus). After specified time, the reaction was stopped and mixture was transferred to an NMR tube. Spectrum of the mixture was measured within 10 minutes after reaction was stopped. 


\section{Crystallographic data}

X-Ray diffraction: Data sets for compound $\mathbf{7}$ were collected with a Bruker APEX II CCD diffractometer. Programs used: data collection: APEX3 V2016.1-0 (Bruker AXS Inc., 2016); cell refinement: SAINT V8.37A (Bruker AXS Inc., 2015); data reduction: SAINT V8.37A (Bruker AXS Inc., 2015); absorption correction, SADABS V2014/7 (Bruker AXS Inc., 2014); structure solution SHELXT-2015; ${ }^{[9]}$ structure refinement SHELXL-2015 ${ }^{[10]}$ and graphics, $X P .^{[11]}$ $R$-values are given for observed reflections, and $w \mathrm{R}^{2}$ values are given for all reflections.

X-ray crystal structure analysis of 7: A colorless prism-like specimen of $\mathrm{C}_{14} \mathrm{H}_{13} \mathrm{BrO}_{2}$, approximate dimensions $0.120 \mathrm{~mm}$ x $0.180 \mathrm{~mm}$ x $0.180 \mathrm{~mm}$, was used for the X-ray crystallographic analysis. The X-ray intensity data were measured. A total of 1134 frames were collected. The total exposure time was 15.58 hours. The frames were integrated with the Bruker SAINT software package using a wide-frame algorithm. The integration of the data using an orthorhombic unit cell yielded a total of 18544 reflections to a maximum $\theta$ angle of $66.60^{\circ}$ (0.84 Å resolution), of which 4187 were independent (average redundancy 4.429, completeness $\left.=99.6 \%, \mathrm{R}_{\text {int }}=5.90 \%, \mathrm{R}_{\text {sig }}=5.22 \%\right)$ and $3732(89.13 \%)$ were greater than $2 \sigma\left(\mathrm{F}^{2}\right)$. The final cell constants of $\underline{a}=27.1951(6) \AA, \underline{b}=7.7918(2) \AA, \underline{c}=11.4425(3) \AA$, volume $=2424.65(10)$ $\AA^{3}$, are based upon the refinement of the XYZ-centroids of 5759 reflections above $20 \sigma(\mathrm{I})$ with $6.500^{\circ}<2 \theta<133.2^{\circ}$. Data were corrected for absorption effects using the multi-scan method (SADABS). The ratio of minimum to maximum apparent transmission was 0.819 . The calculated minimum and maximum transmission coefficients (based on crystal size) are 0.4970 and 0.6140. The structure was solved and refined using the Bruker SHELXTL Software Package, using the space group $P c a 2_{1}$, with $\mathrm{Z}=8$ for the formula unit, $\mathrm{C}_{14} \mathrm{H}_{13} \mathrm{BrO}_{2}$. The final anisotropic full-matrix least-squares refinement on $\mathrm{F}^{2}$ with 307 variables converged at $\mathrm{R} 1=$ $4.79 \%$, for the observed data and wR2 $=12.59 \%$ for all data. The goodness-of-fit was 1.088 . The largest peak in the final difference electron density synthesis was $1.476 \mathrm{e}^{-} / \AA^{3}$ and the largest hole was $-0.707 \mathrm{e}^{-} / \AA^{3}$ with an RMS deviation of $0.091 \mathrm{e}^{-} / \AA^{3}$. On the basis of the final model, the calculated density was $1.606 \mathrm{~g} / \mathrm{cm}^{3}$ and F(000), $1184 \mathrm{e}^{-}$. CCDC number: 1960689. 


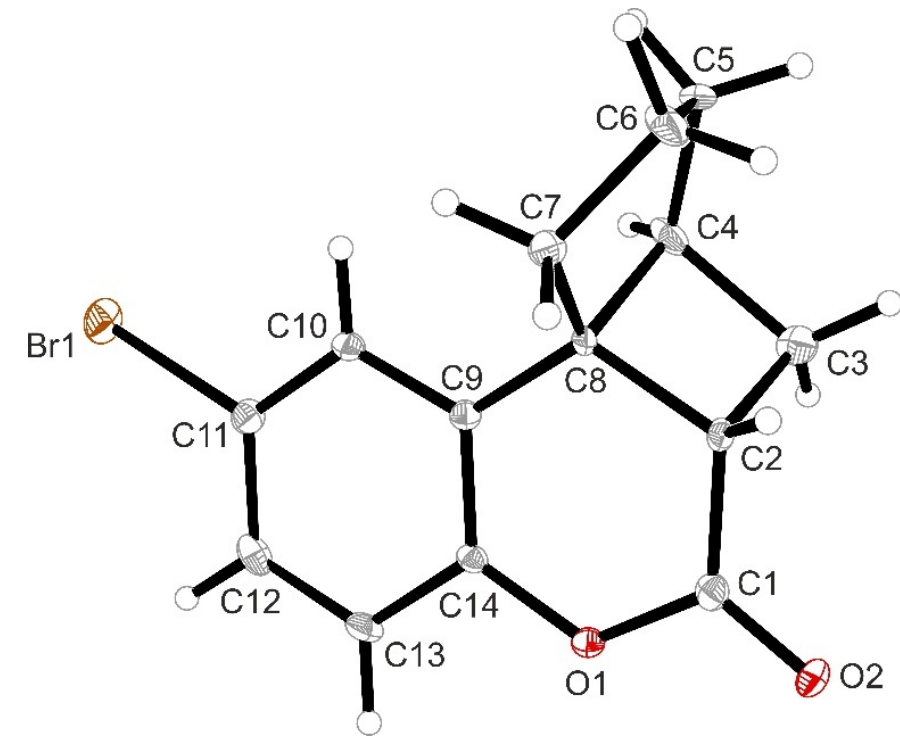

Figure S1: Crystal structure of compound 7. Only one molecule (molecule "B") of two found in the asymmetric unit is shown. Thermal ellipsoids are set at 15\% probability. 


\section{References}

(1) Watanabe, K.; Mino, T.; Hatta, C.; Ishikawa, E.; Yoshida, Y.; Sakamoto, M. Hydrazone-Cu-Catalyzed Suzuki-Miyaura-Type Reactions of Dibromoalkenes with Arylboronic Acids. Eur. J. Org. Chem. 2017, 2017 (25), 3612-3619.

(2) Kikushima, K.; Moriuchi, T.; Hirao, T. Oxidative Bromination Reaction Using Vanadium Catalyst and Aluminum Halide under Molecular Oxygen. Tetrahedron Lett. 2010, 51 (2), 340-342.

(3) Han, X.; Xia, Q.; Huang, J.; Liu, Y.; Tan, C.; Cui, Y. Chiral Covalent Organic Frameworks with High Chemical Stability for Heterogeneous Asymmetric Catalysis. J. Am. Chem. Soc. 2017, 139 (25), 8693-8697.

(4) Liu, F.; Zhong, J.; Zhou, Y.; Gao, Z.; Walsh, P. J.; Wang, X.; Ma, S.; Hou, S.; Liu, S.; Wang, M.; Wang, M.; Bian, Q. Cobalt-Catalyzed Enantioselective Negishi Cross-Coupling of Racemic $\alpha$-Bromo Esters with Arylzincs. Chem.: Eur. J. 2018, 24 (9), 2059-2064.

(5) Álvarez-Calero, J. M.; Jorge, Z. D.; Massanet, G. M. TiCl4/Et3N-Mediated Condensation of Acetate and Formate Esters: Direct Access to $\beta$-Alkoxy- and $\beta$ Aryloxyacrylates. Org. Lett. 2016, 18 (24), 6344-6347.

(6) Gärtner, D.; Stein, A. L.; Grupe, S.; Arp, J.; Jacobi Von Wangelin, A. IronCatalyzed Cross-Coupling of Alkenyl Acetates. Angew. Chem. Int. Ed. 2015, 54 (36), 10545-10549.

(7) Li, C.; Vasella, A. Intramolecular Addition of Nucleophilic Carbenes to Acceptor-Substituted Alkenyl Groups: Synthesis and Transformation of Homobenzofurans and Synthesis of a Homoindole. Helv. Chim. Acta 1993, 76 (1), 197-210.

(8) Brimioulle, R.; Guo, H.; Bach, T. Enantioselective Intramolecular [2+2] Photocycloaddition Reactions of 4-Substituted Coumarins Catalyzed by a Chiral Lewis Acid. Chem.: Eur. J. 2012, 18 (24), 7552-7560.

(9) Sheldrick, G. M., SHELXT - Integrated space-group and crystal-structure determination. Acta Cryst., 2015, A71, 3-8.

(10) Sheldrick, G.M., Crystal structure refinement with SHELXL, Acta Cryst., 2015, C71 (1), 3-8.

(11) XP - Interactive molecular graphics, Version 5.1, Bruker AXS Inc., Madison, Wisconsin, USA, 1998. 
NOESY of key signals in compounds 2, 13, 13-Z

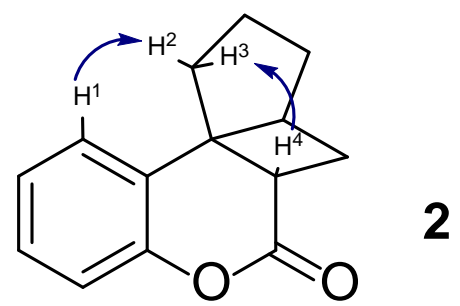
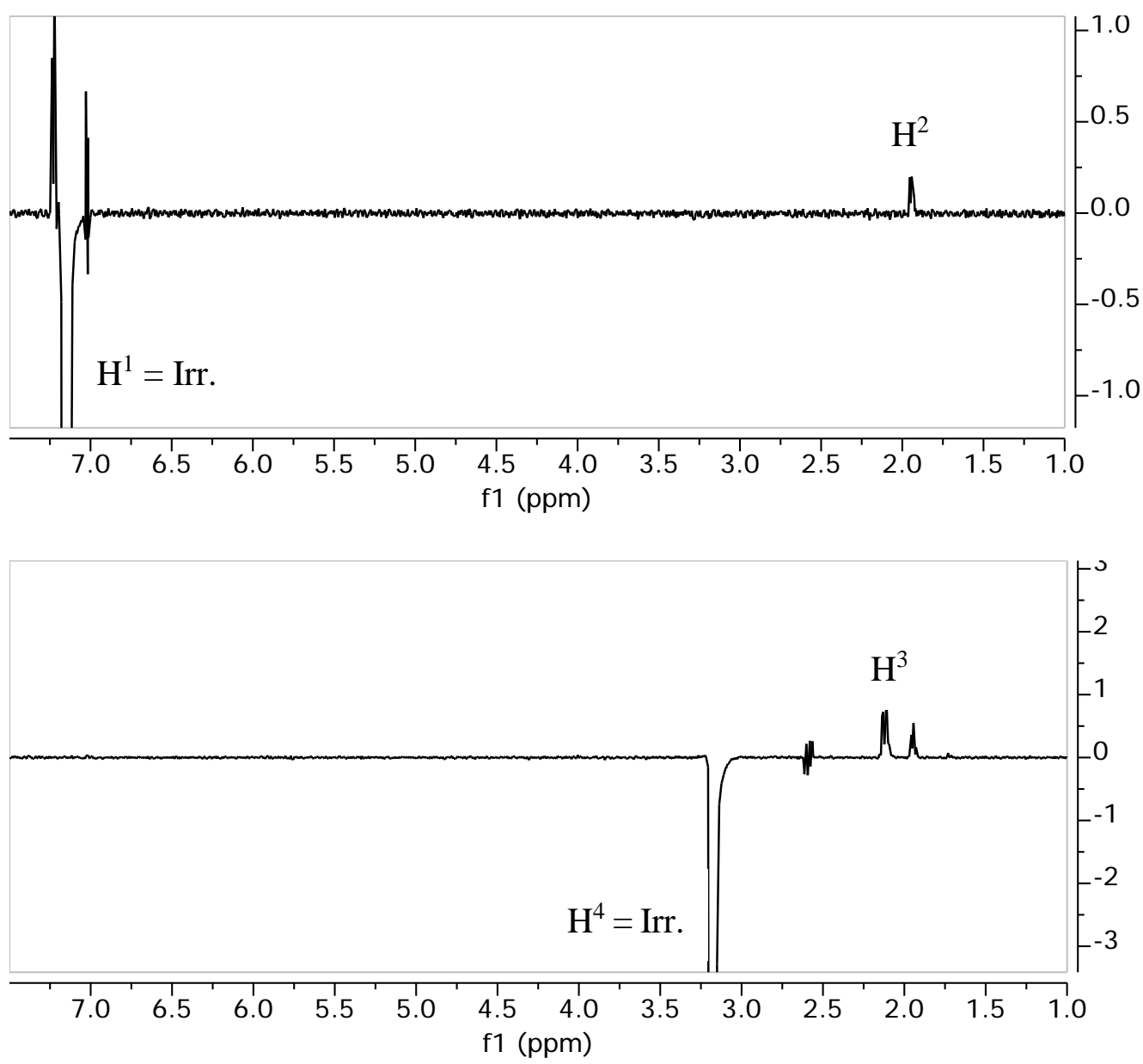
<smiles>C=CCCC/C(C(=O)OCC)=C1\CC[C@@H]2Oc3ccccc3[C@@H](CC1)O2</smiles>

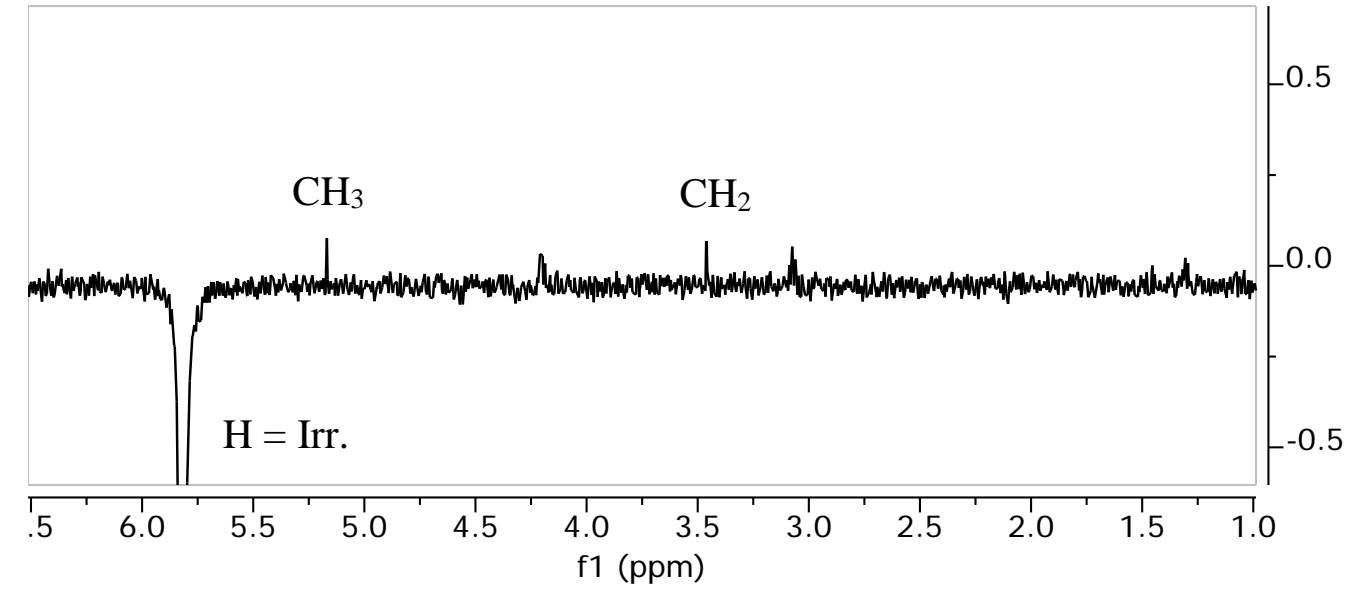




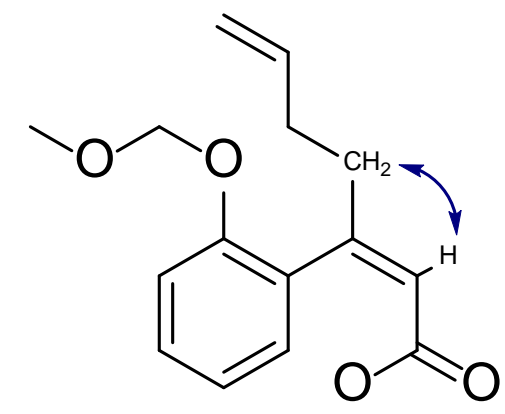

\section{3-Z}
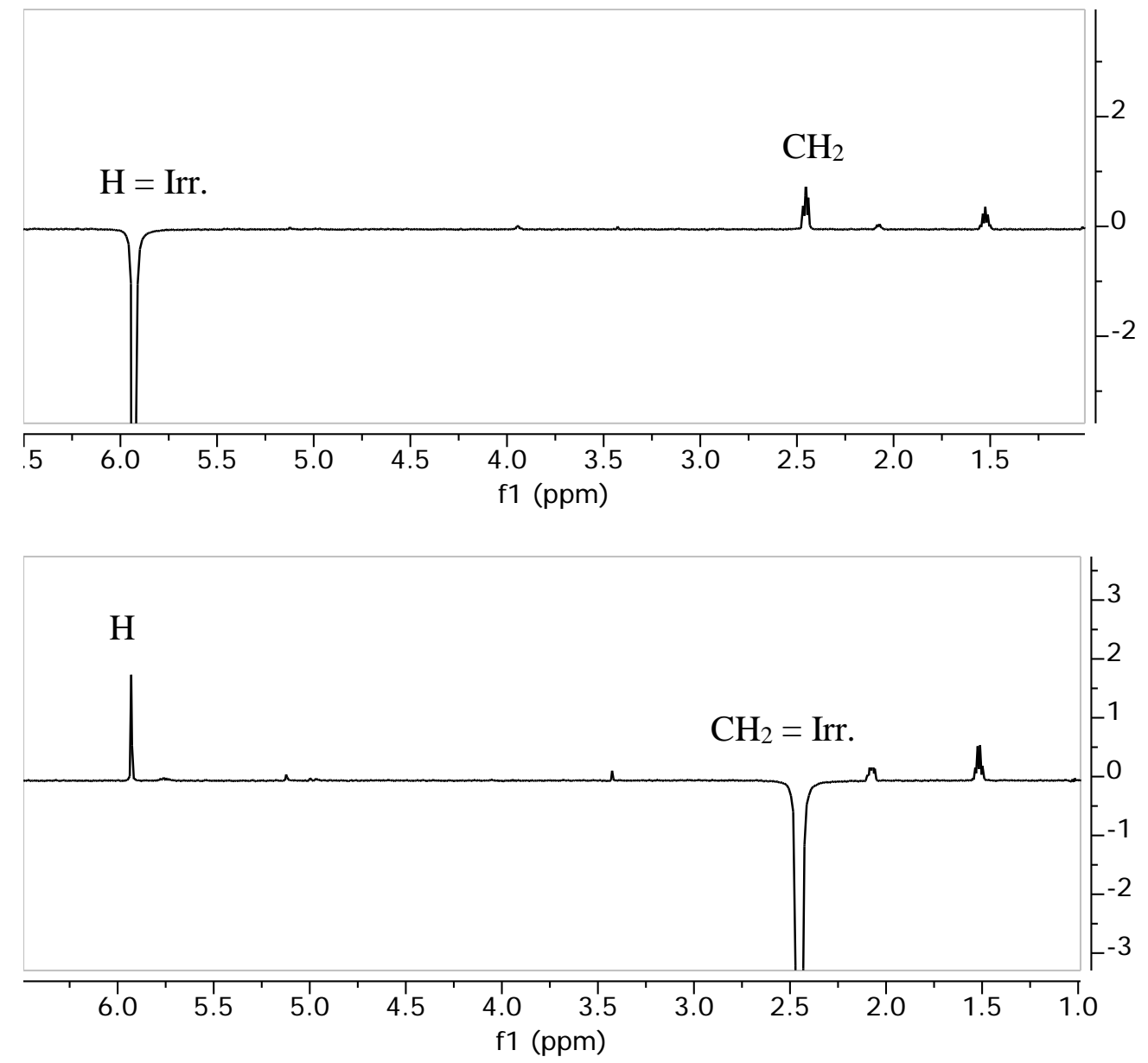
NMR-Spectra of Key Compounds

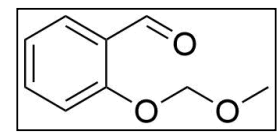

\section{S-1}

${ }^{1} \mathrm{H}$ NMR (300 MHz, $\mathrm{CDCl}_{3}$ )

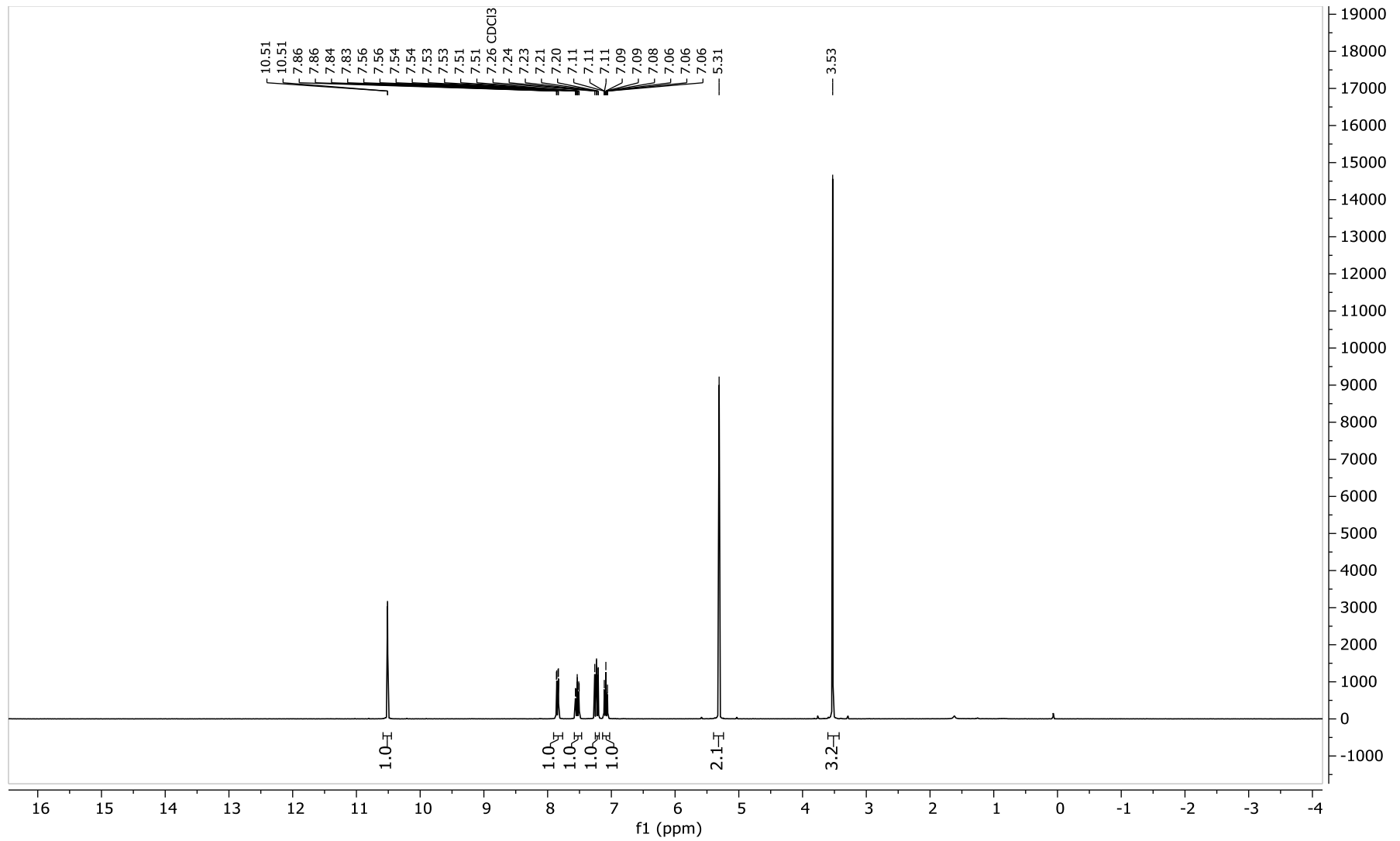

${ }^{13} \mathrm{C}$ NMR $\left(75 \mathrm{MHz}, \mathrm{CDCl}_{3}\right)$

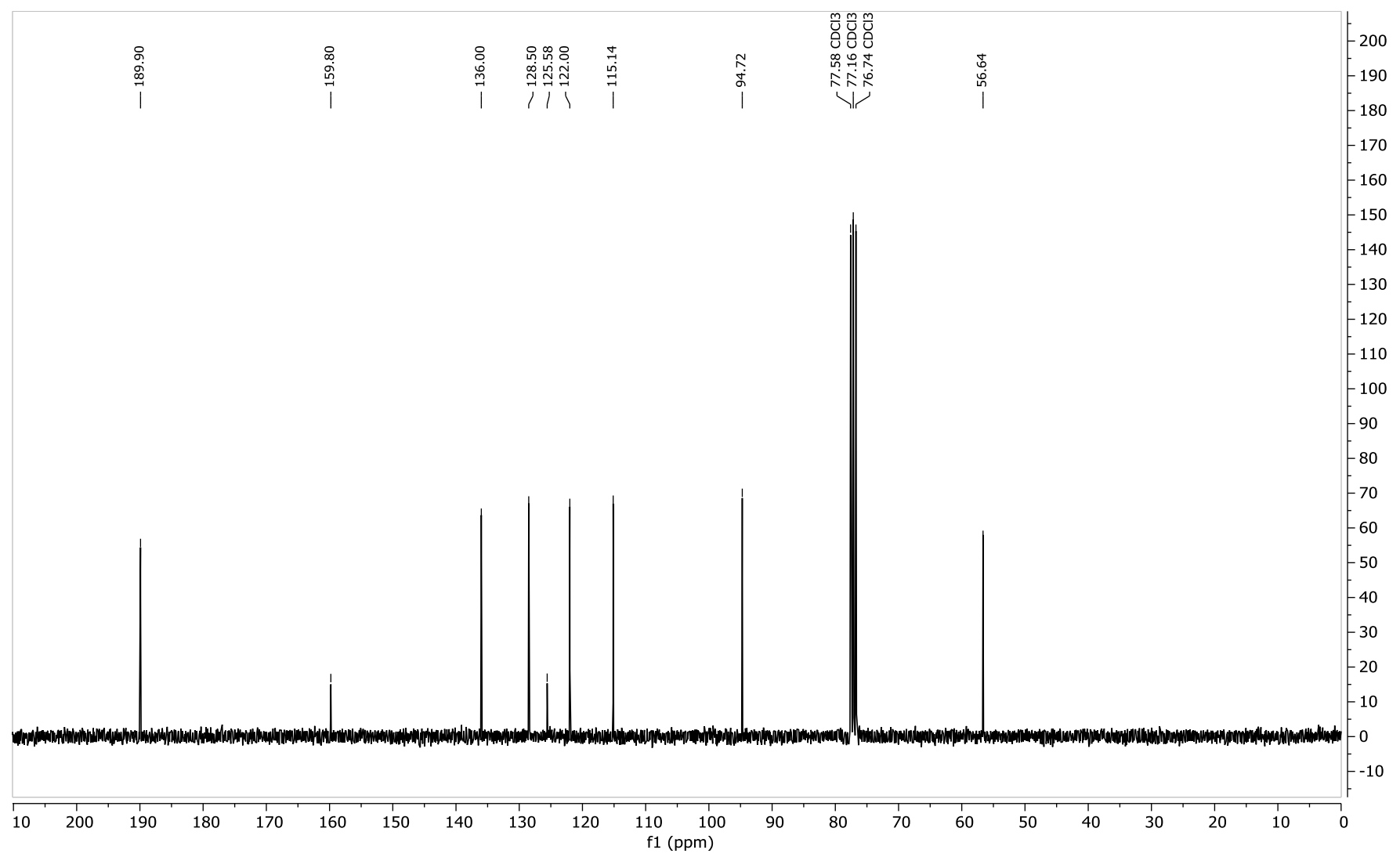




\section{S-2}

${ }^{1} \mathrm{H}$ NMR $\left(300 \mathrm{MHz}, \mathrm{CDCl}_{3}\right)$

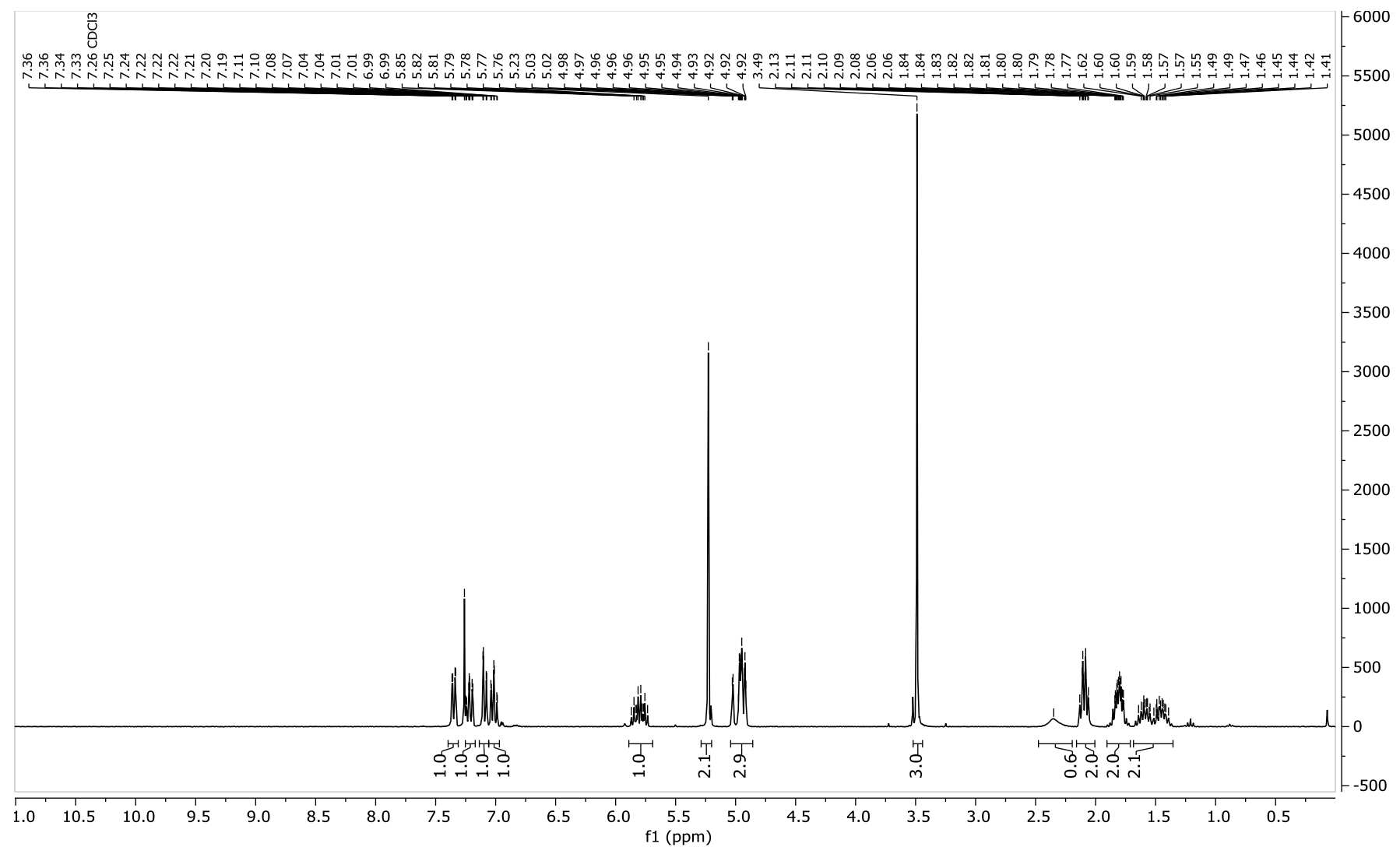

${ }^{13} \mathrm{C}$ NMR $\left(75 \mathrm{MHz}, \mathrm{CDCl}_{3}\right)$

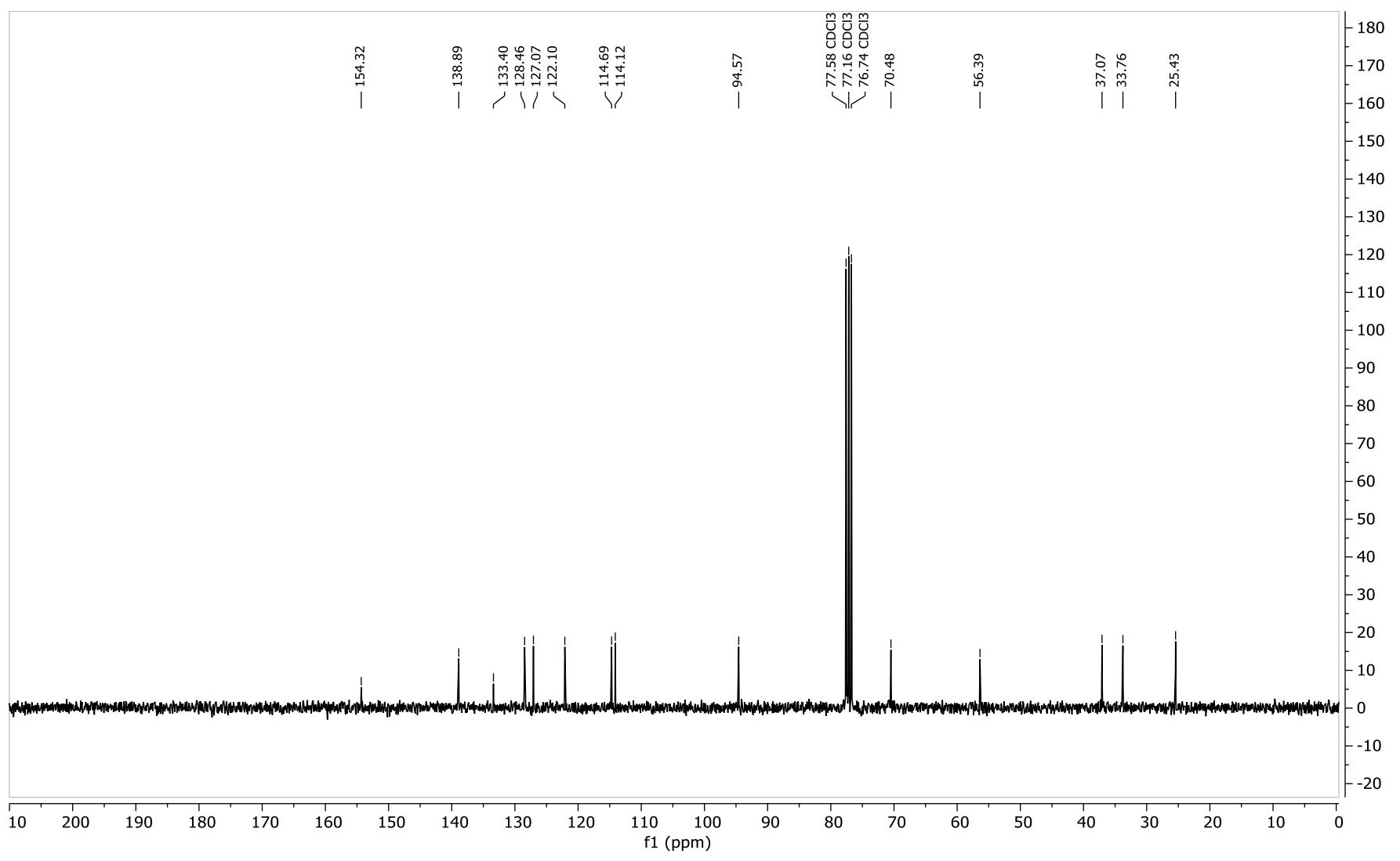




\section{S-3}

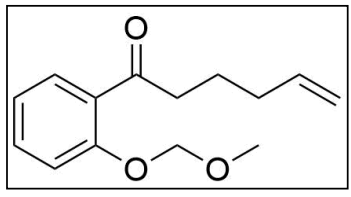

${ }^{1} \mathrm{H}$ NMR $\left(400 \mathrm{MHz}, \mathrm{CDCl}_{3}\right)$

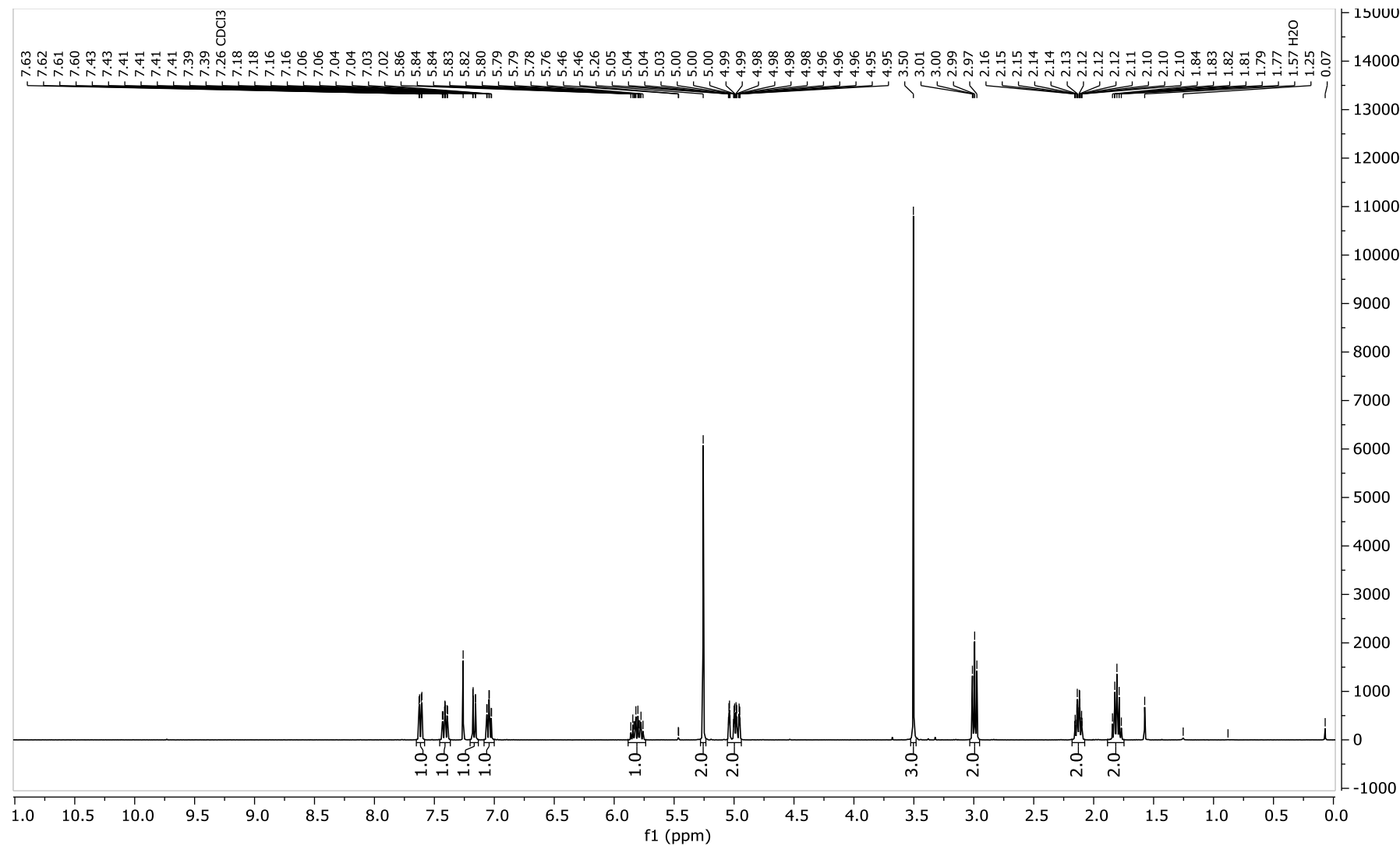

${ }^{13} \mathrm{C}$ NMR (101 MHz, $\mathrm{CDCl}_{3}$ )

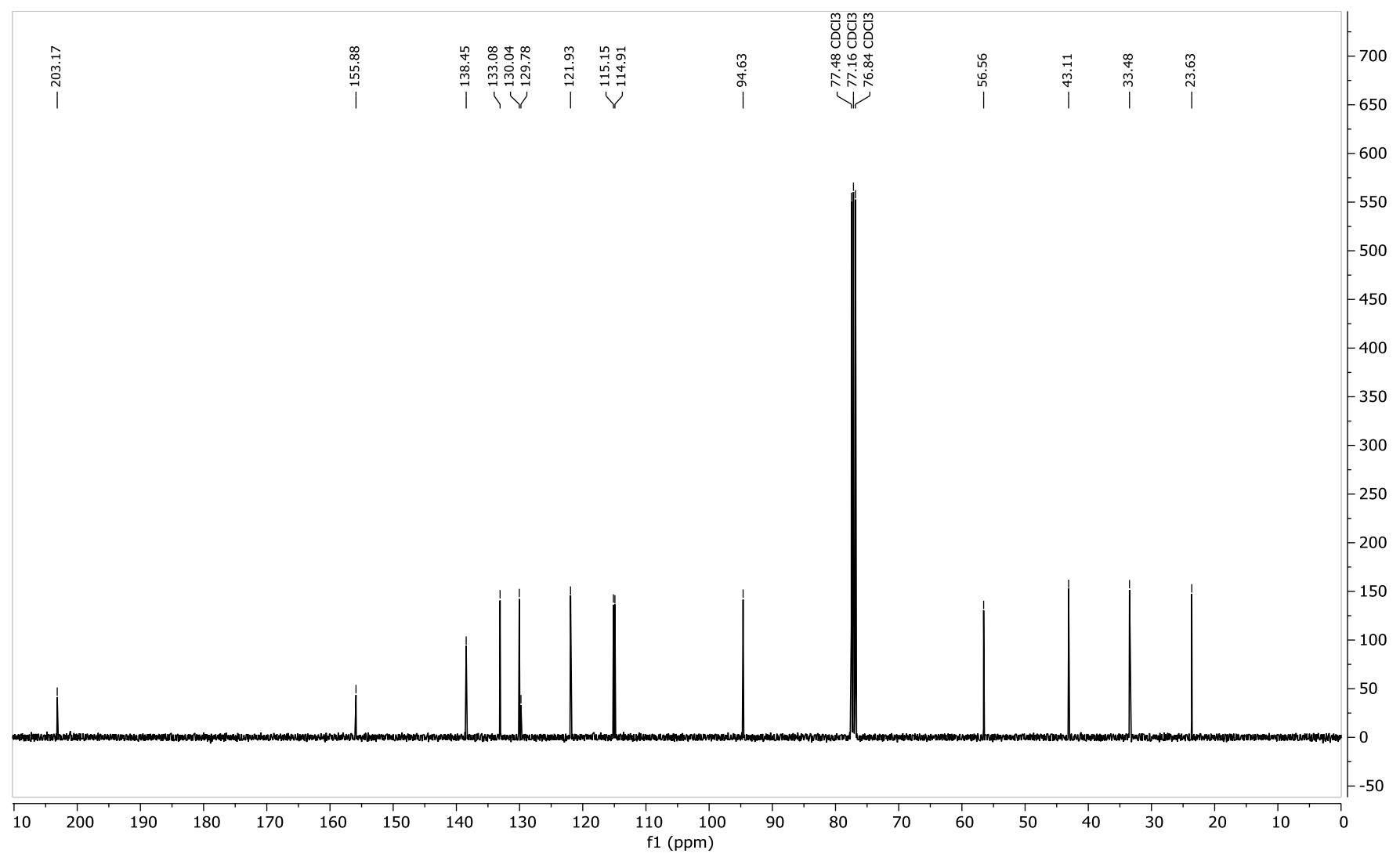


${ }^{1} \mathrm{H}$ NMR (400 MHz, $\left.\mathrm{CDCl}_{3}\right)$

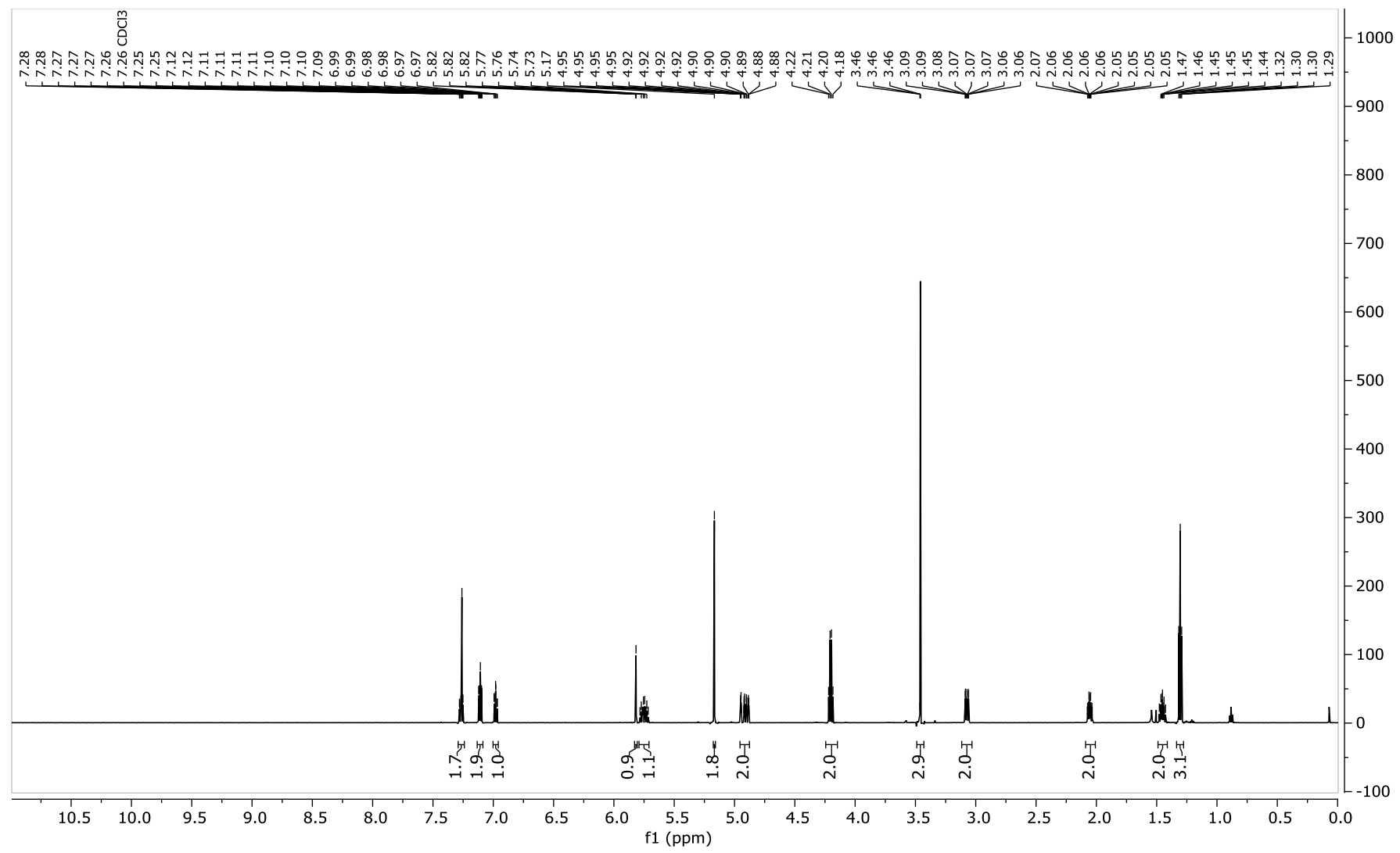

${ }^{13} \mathrm{C}$ NMR (101 MHz, $\mathrm{CDCl}_{3}$ )

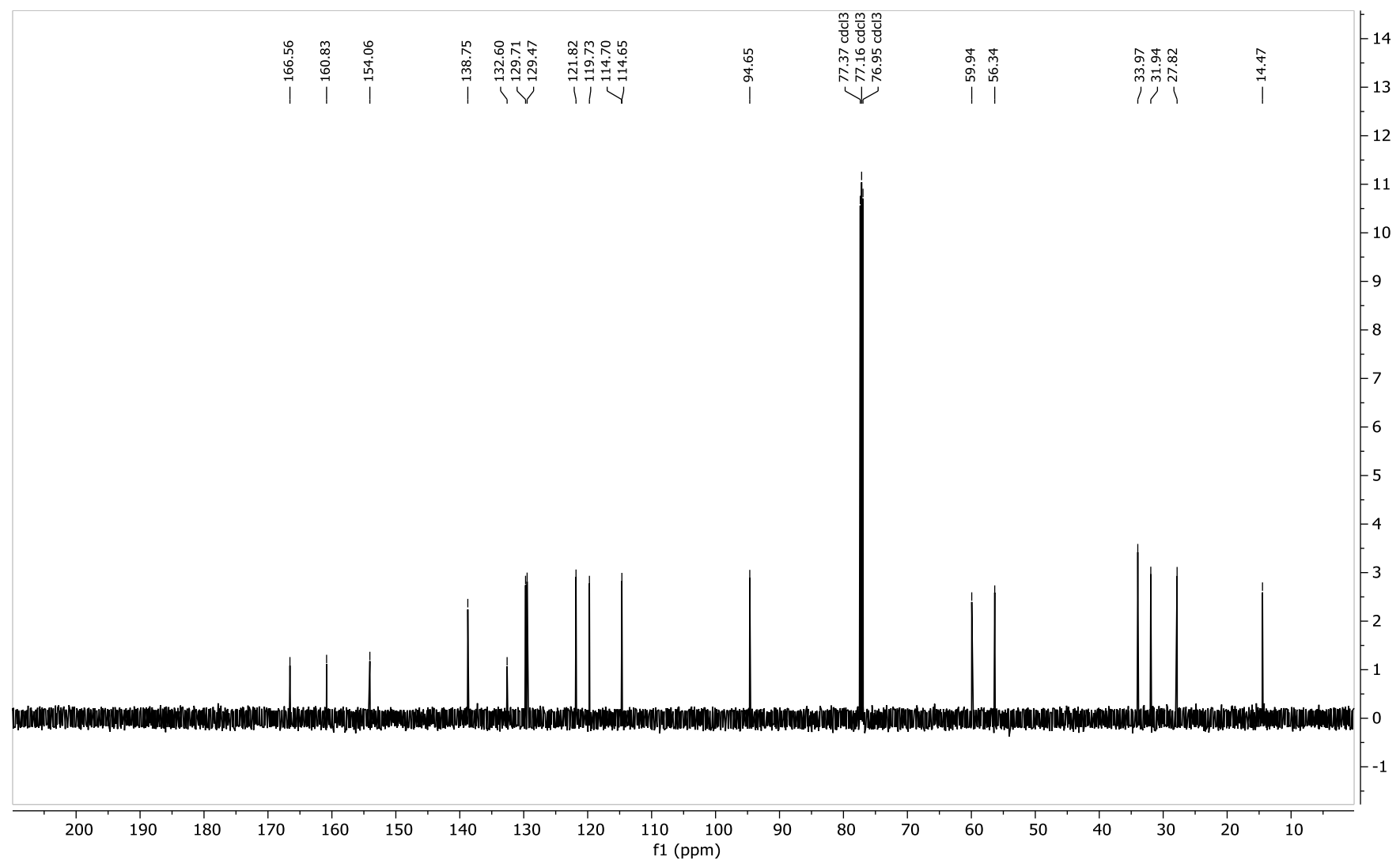




\section{3-Z}

${ }^{1} \mathrm{H}$ NMR $\left(599 \mathrm{MHz}, \mathrm{CDCl}_{3}\right.$ )

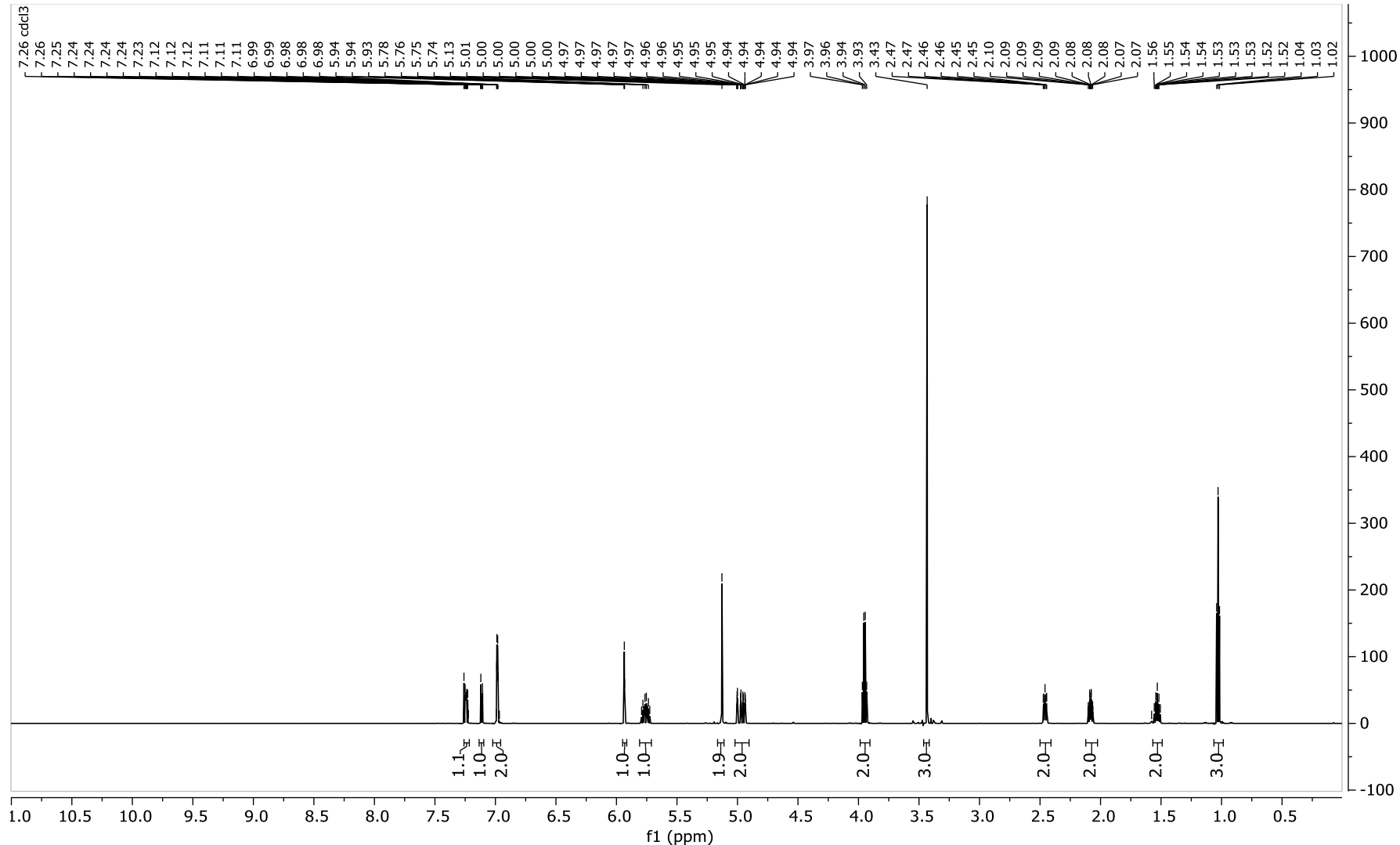

${ }^{13} \mathrm{C}$ NMR $\left(151 \mathrm{MHz}, \mathrm{CDCl}_{3}\right)$

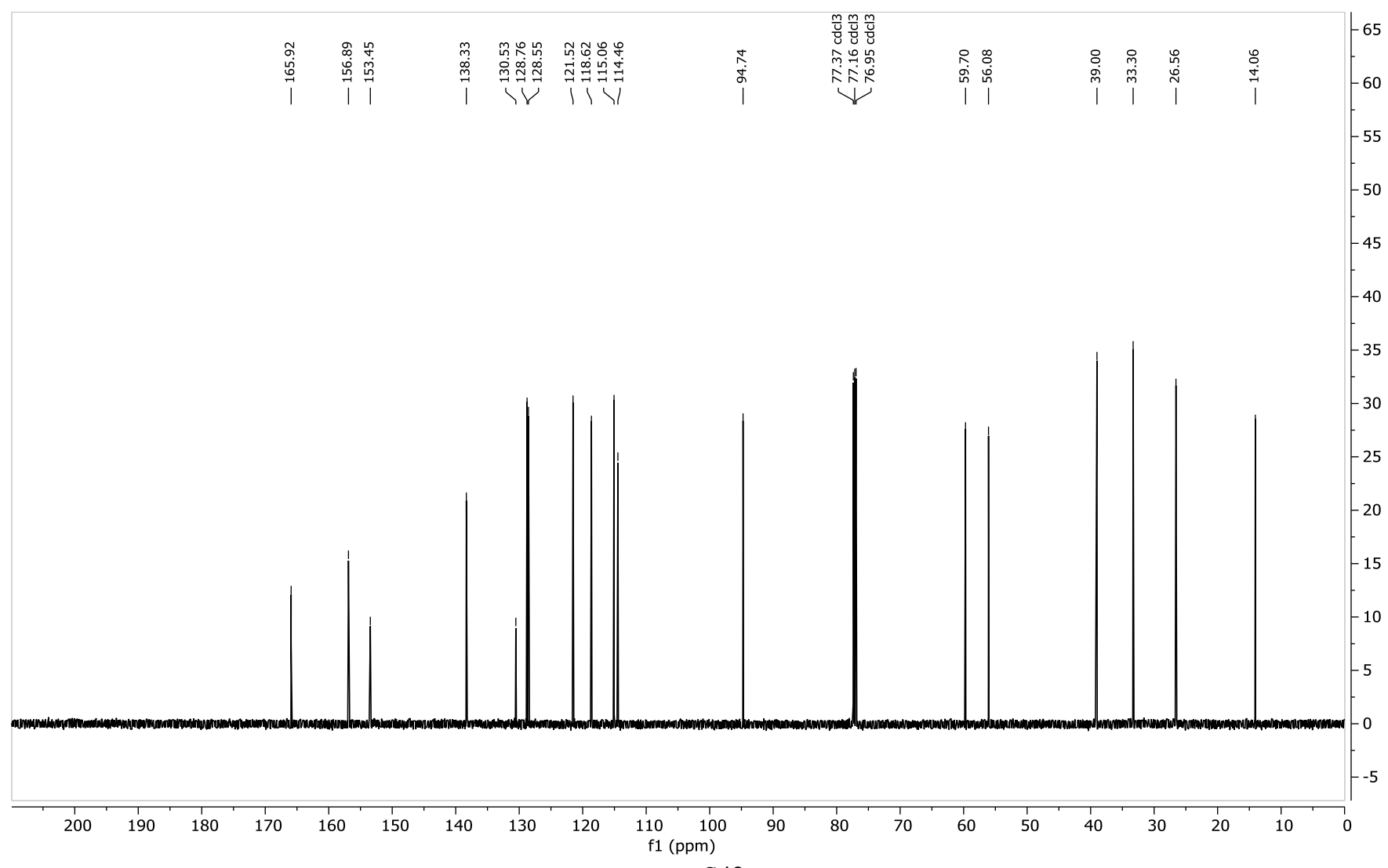




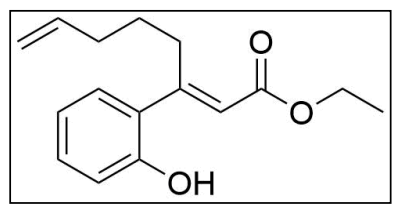

${ }^{1} \mathrm{H}$ NMR $\left(300 \mathrm{MHz}, \mathrm{CDCl}_{3}\right)$

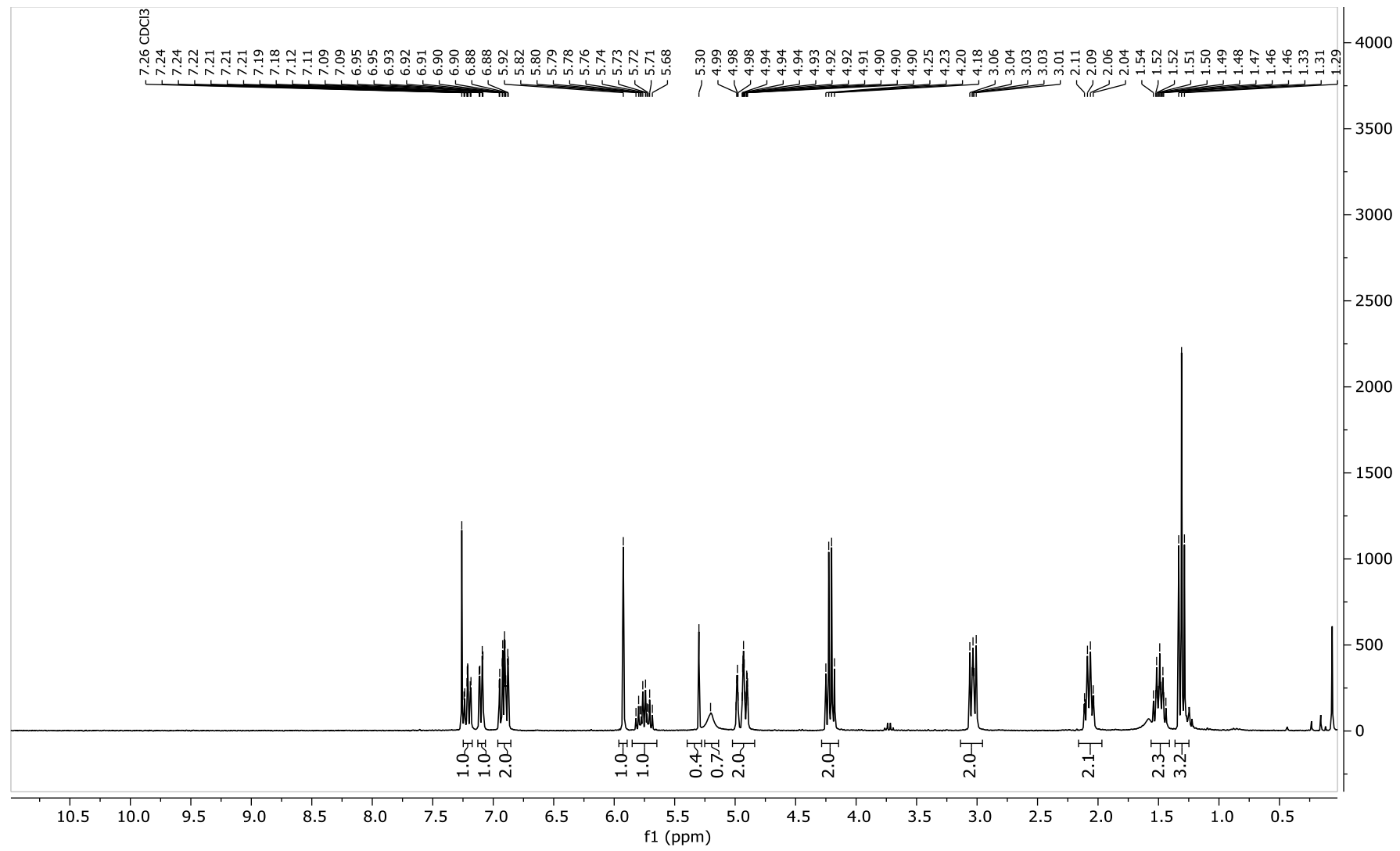

${ }^{13} \mathrm{C} \mathrm{NMR}\left(75 \mathrm{MHz}, \mathrm{CDCl}_{3}\right)$

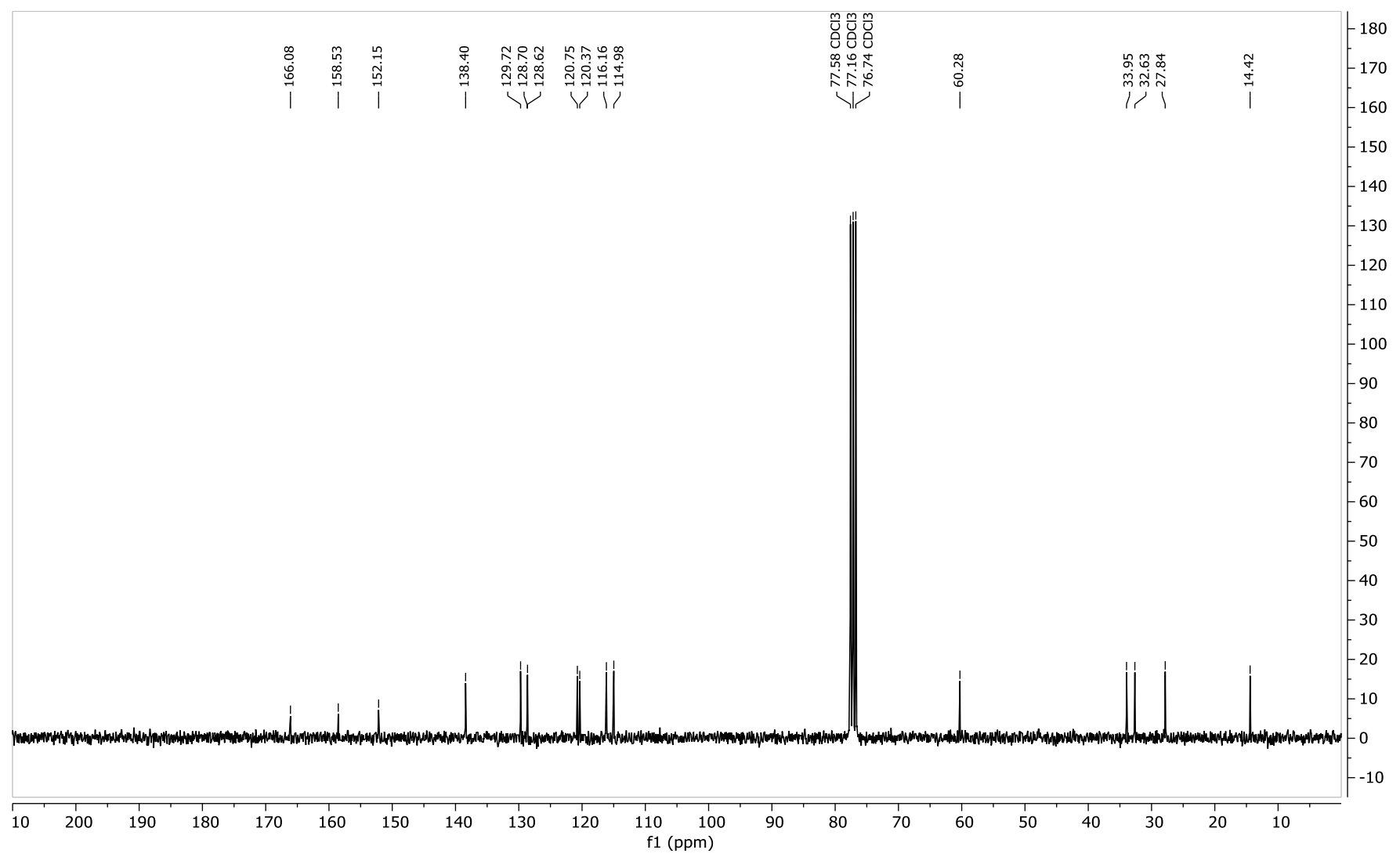




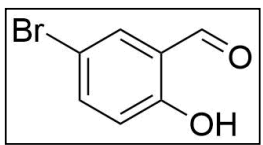

\section{S-4}

${ }^{1} \mathrm{H}$ NMR $\left(300 \mathrm{MHz}, \mathrm{CDCl}_{3}\right)$

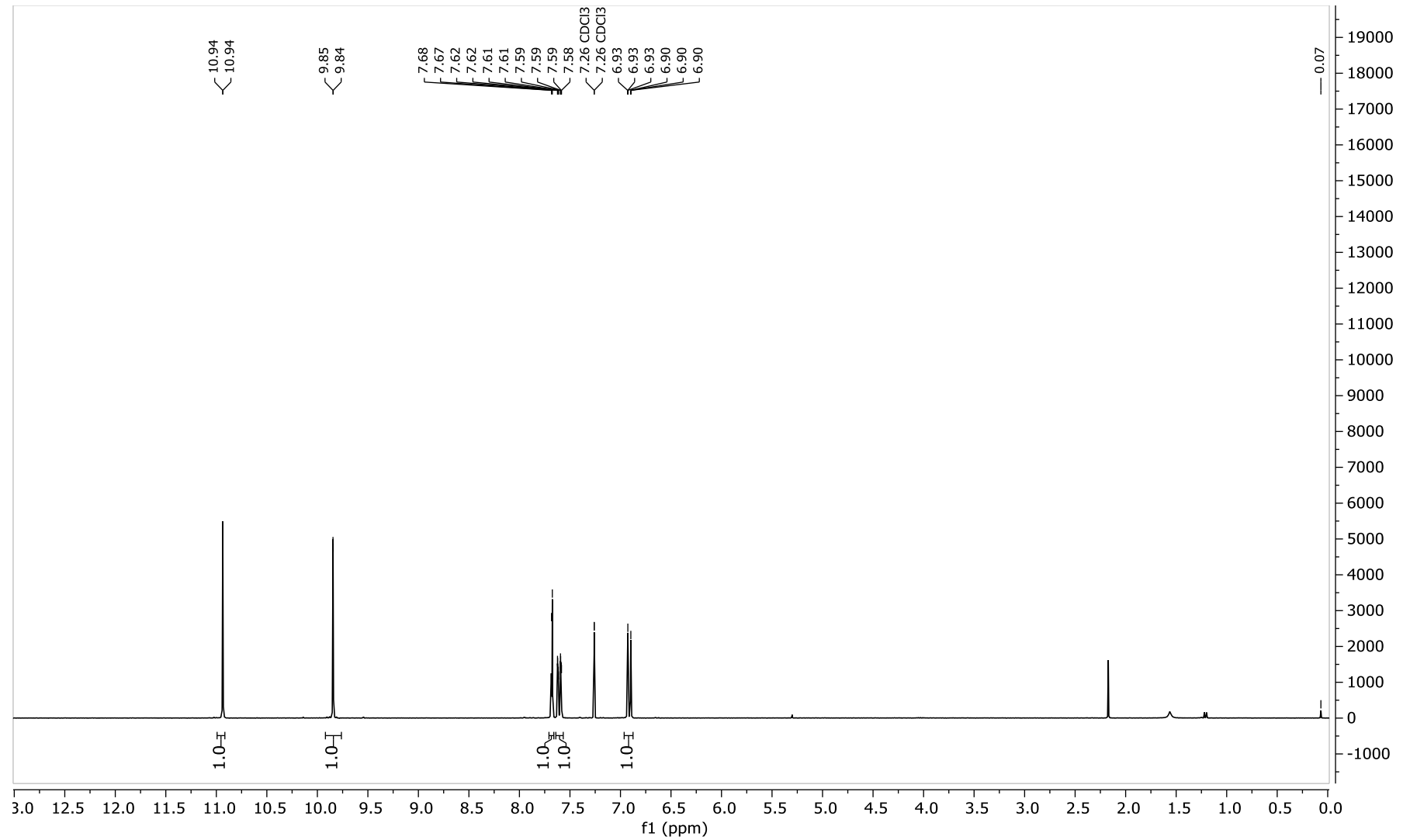

${ }^{13} \mathrm{C} \mathrm{NMR}\left(75 \mathrm{MHz}, \mathrm{CDCl}_{3}\right)$

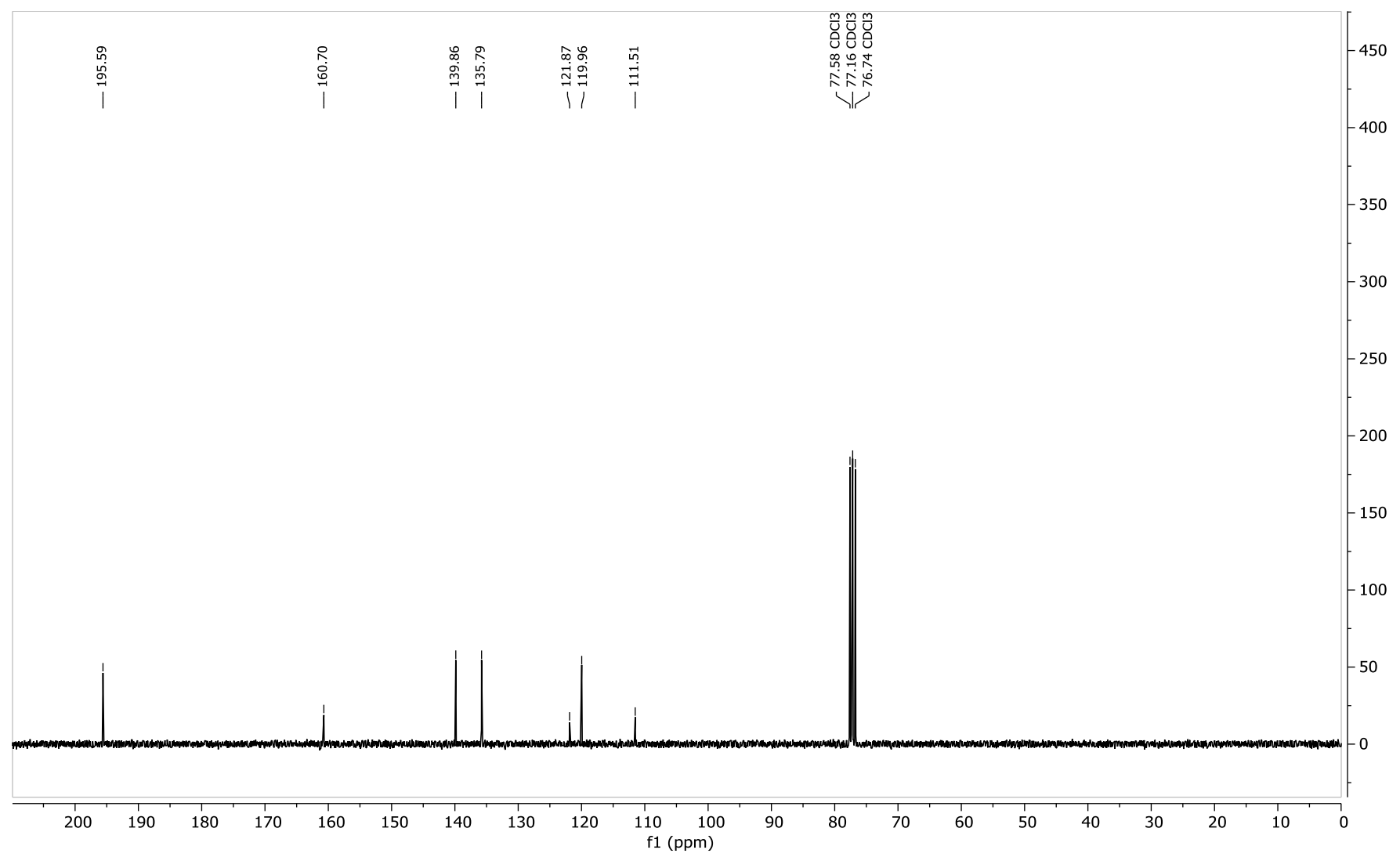




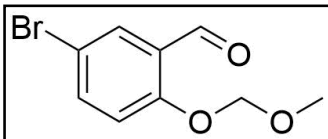

\section{S-5}

${ }^{1} \mathrm{H}$ NMR $\left(400 \mathrm{MHz}, \mathrm{CDCl}_{3}\right)$

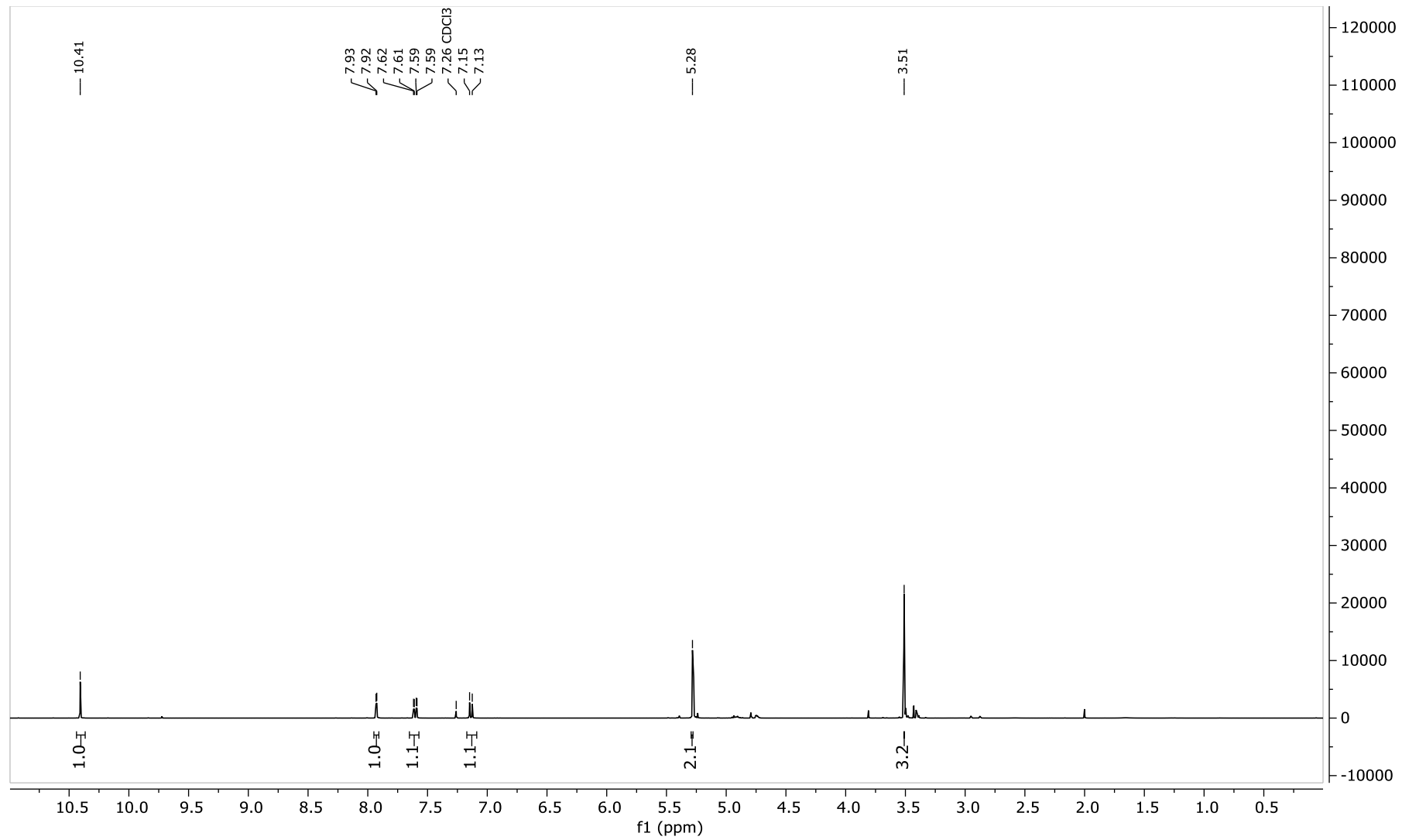

${ }^{13} \mathrm{C}$ NMR (101 MHz, $\mathrm{CDCl}_{3}$ )

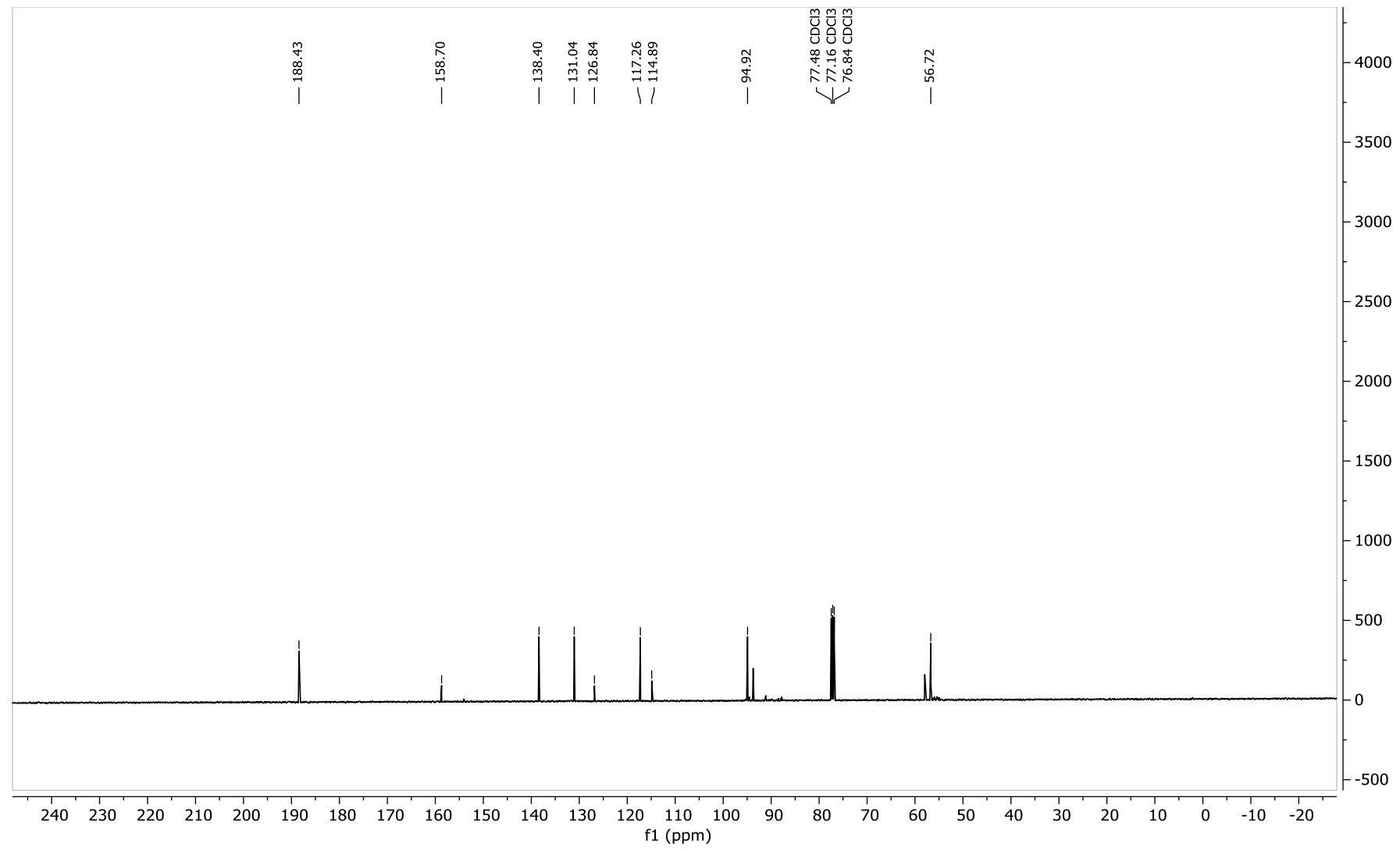




\section{S-6}

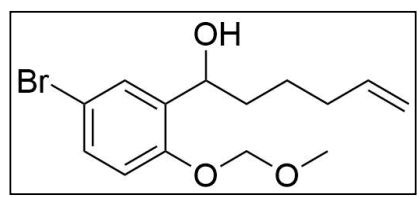

${ }^{1} \mathrm{H}$ NMR $\left(300 \mathrm{MHz}, \mathrm{CDCl}_{3}\right)$

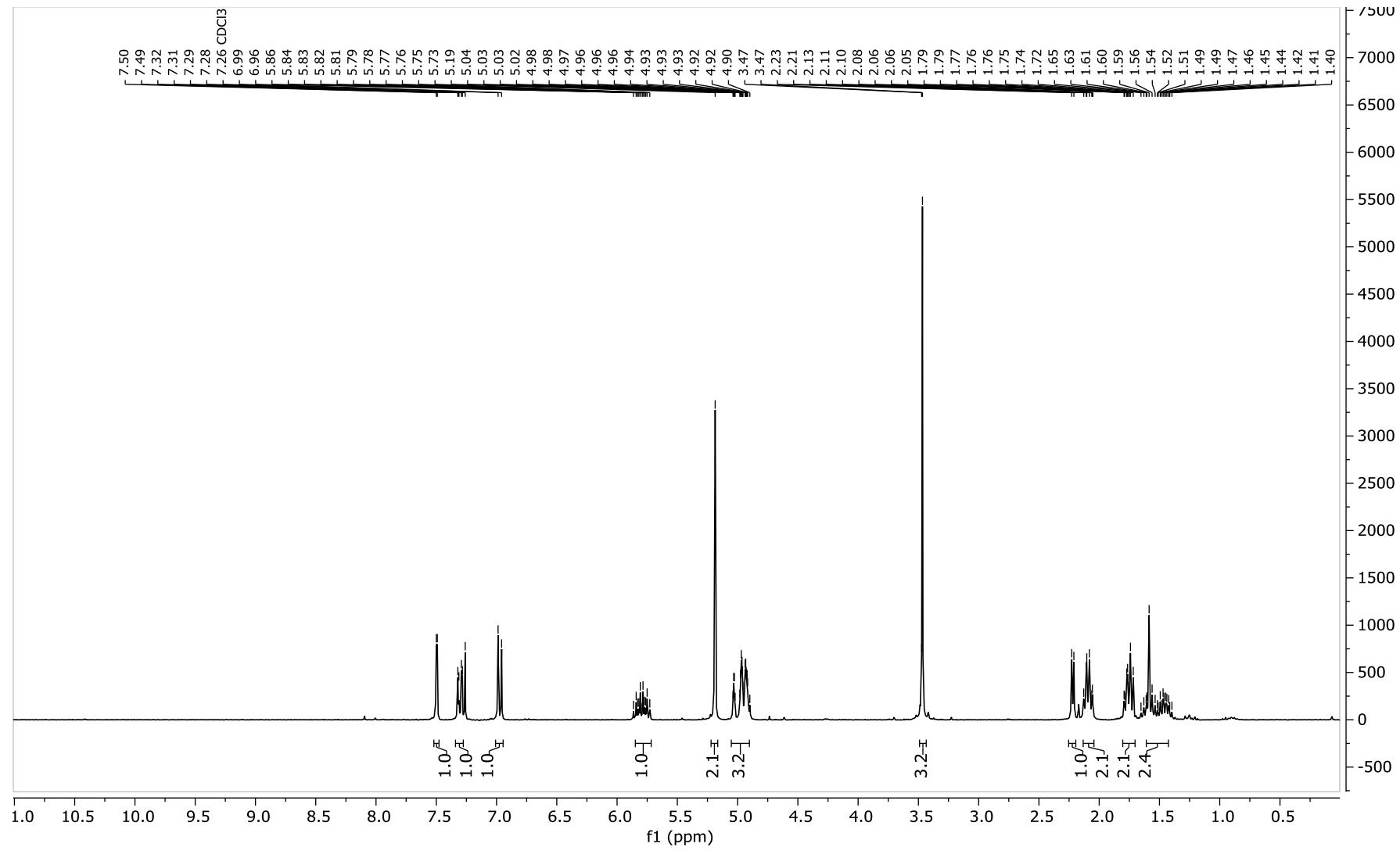

${ }^{13} \mathrm{C} \mathrm{NMR}\left(75 \mathrm{MHz}, \mathrm{CDCl}_{3}\right)$

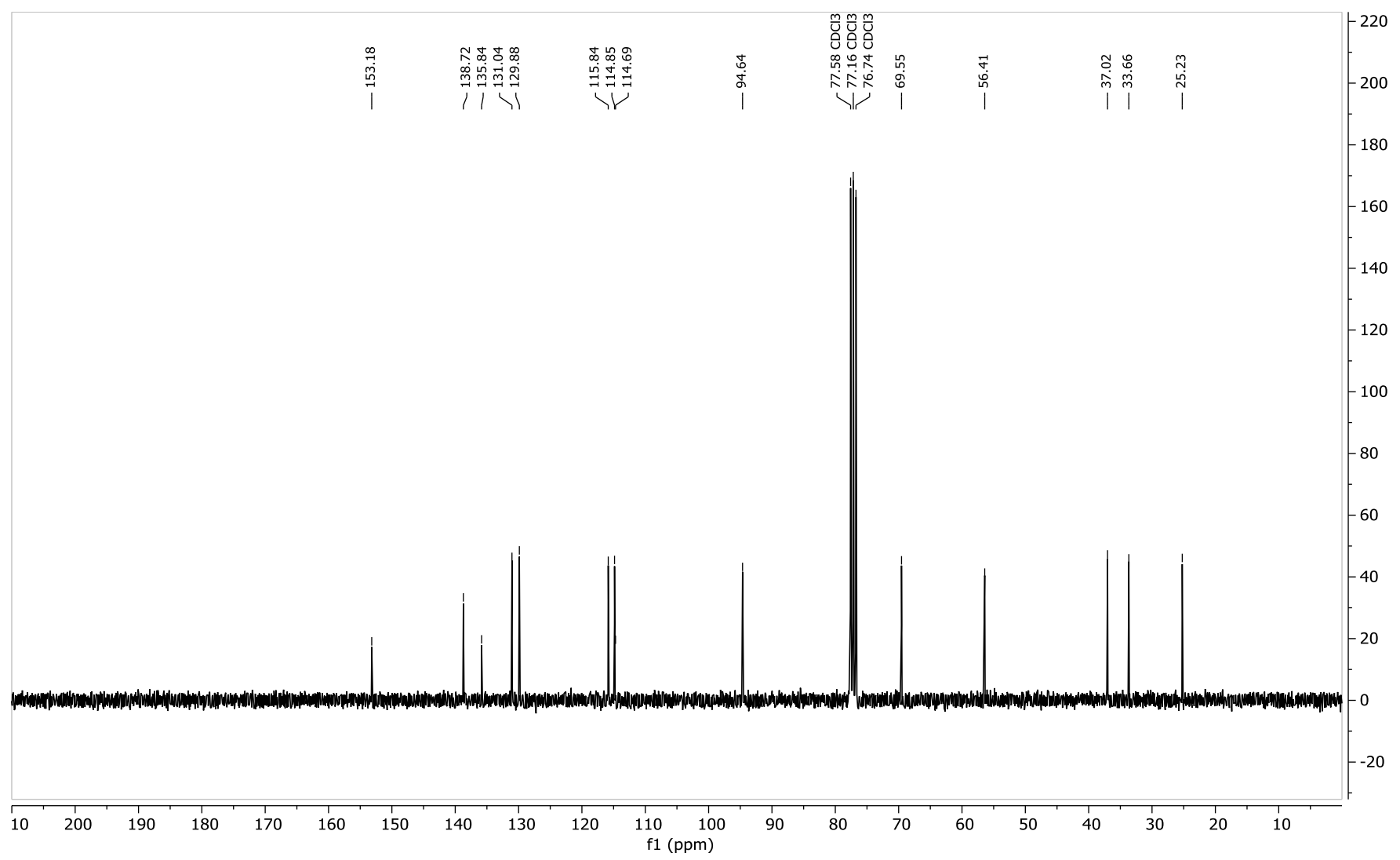




\section{S-7}

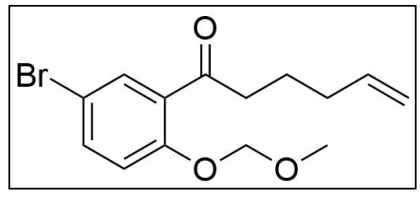

${ }^{1} \mathrm{H}$ NMR $\left(400 \mathrm{MHz}, \mathrm{CDCl}_{3}\right)$

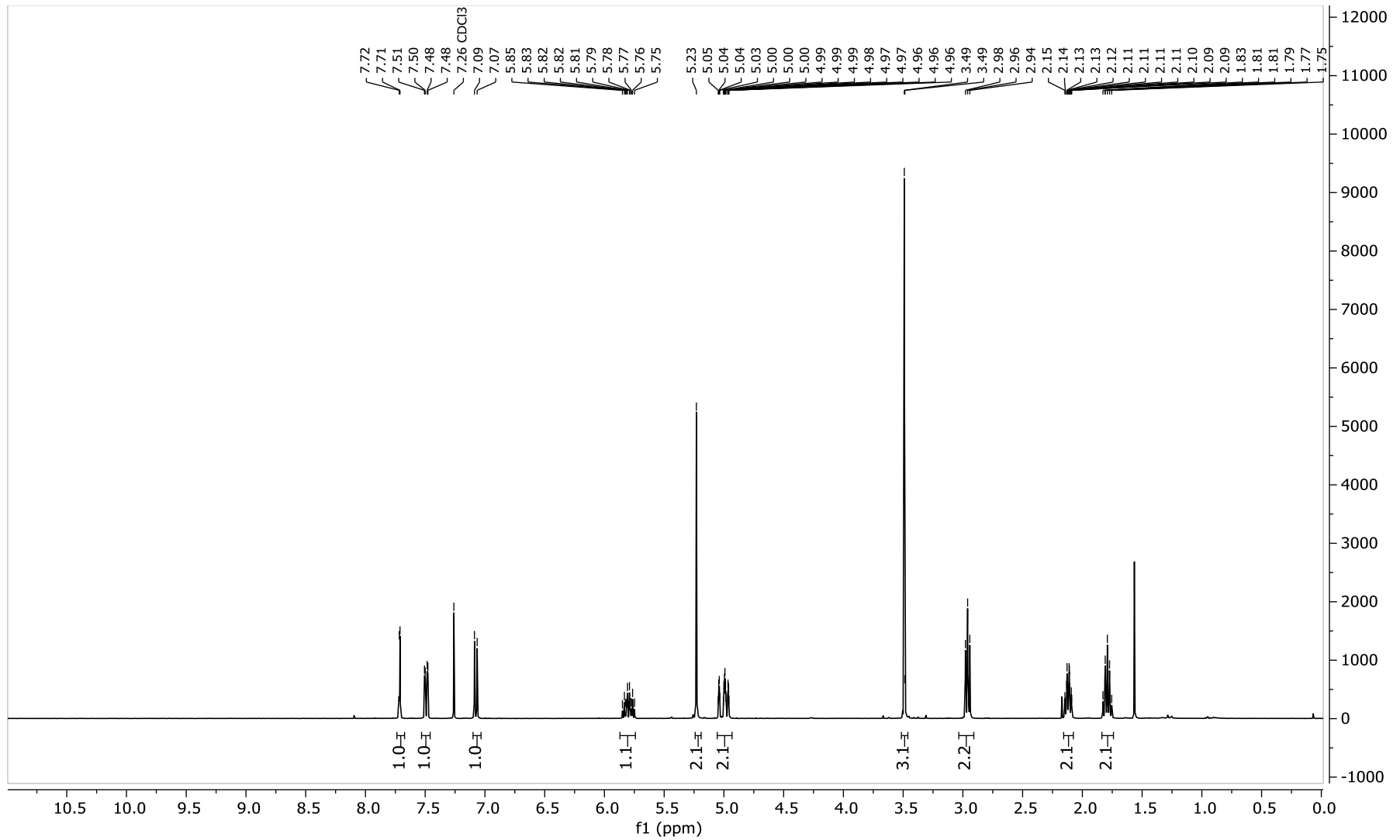

${ }^{13} \mathrm{C}$ NMR (101 MHz, $\mathrm{CDCl}_{3}$ )

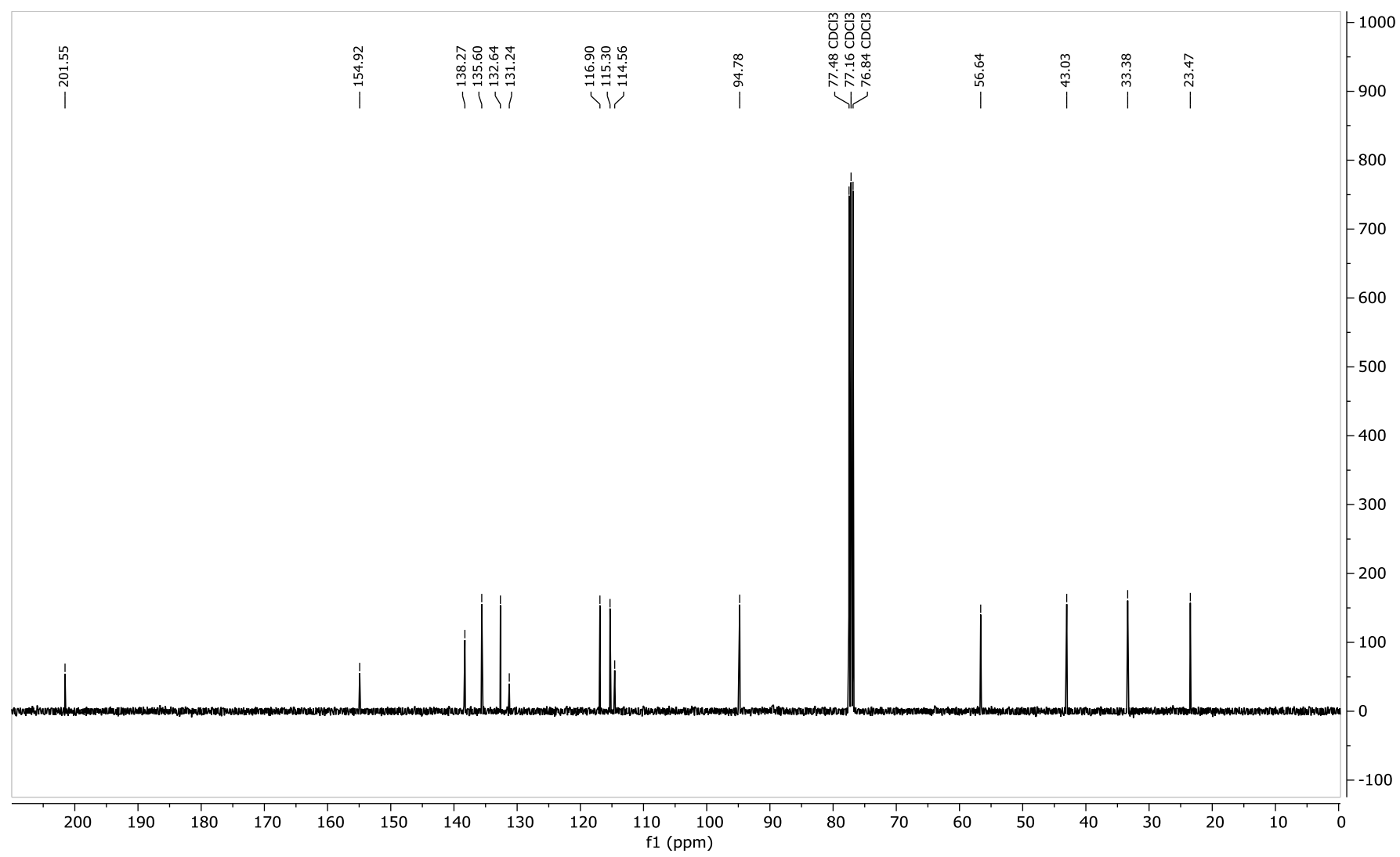




\section{S-8}

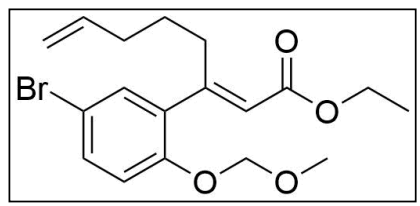

${ }^{1} \mathrm{H}$ NMR $\left(500 \mathrm{MHz}, \mathrm{CDCl}_{3}\right)$

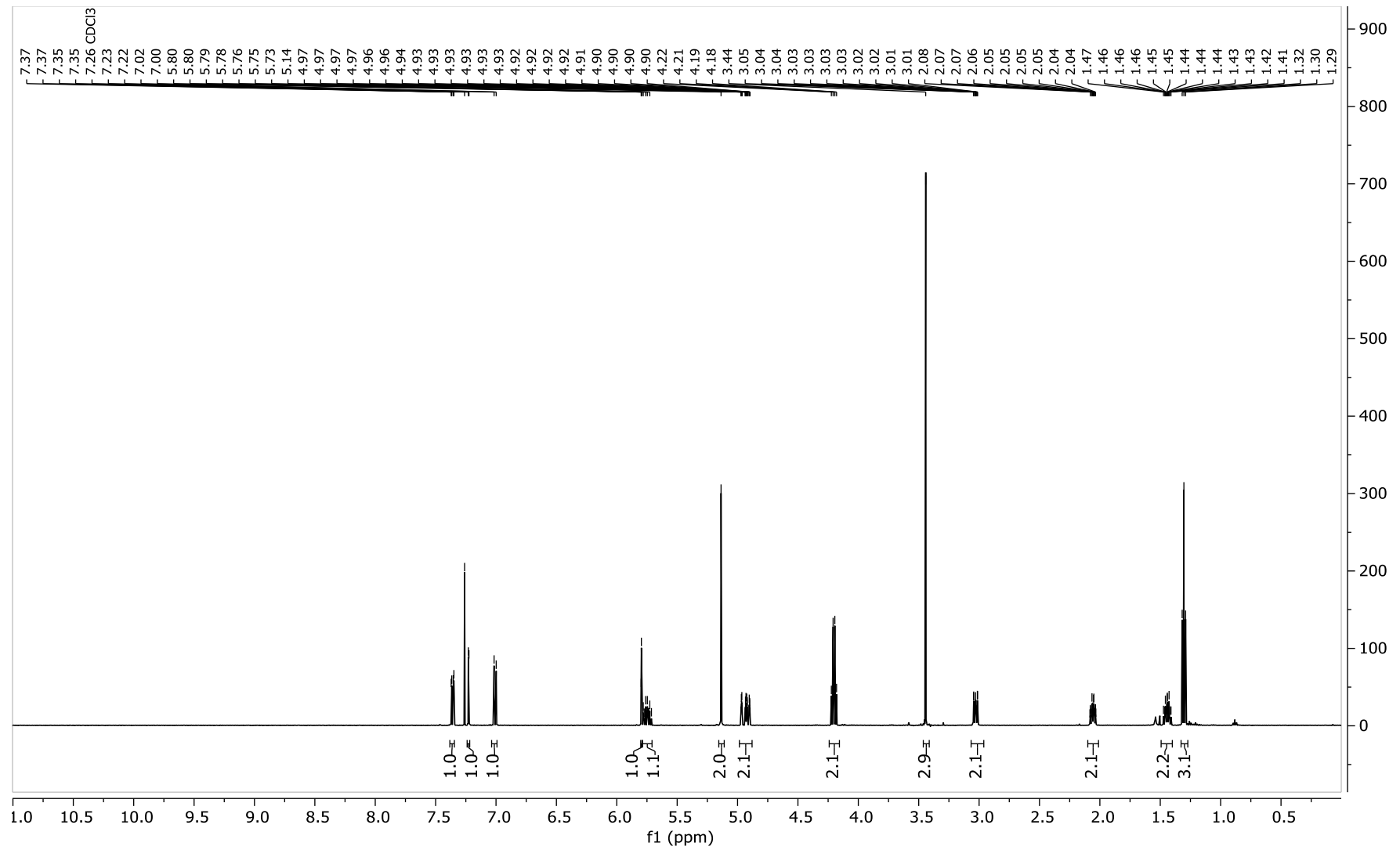

${ }^{13} \mathrm{C}$ NMR (126 MHz, $\mathrm{CDCl}_{3}$ )

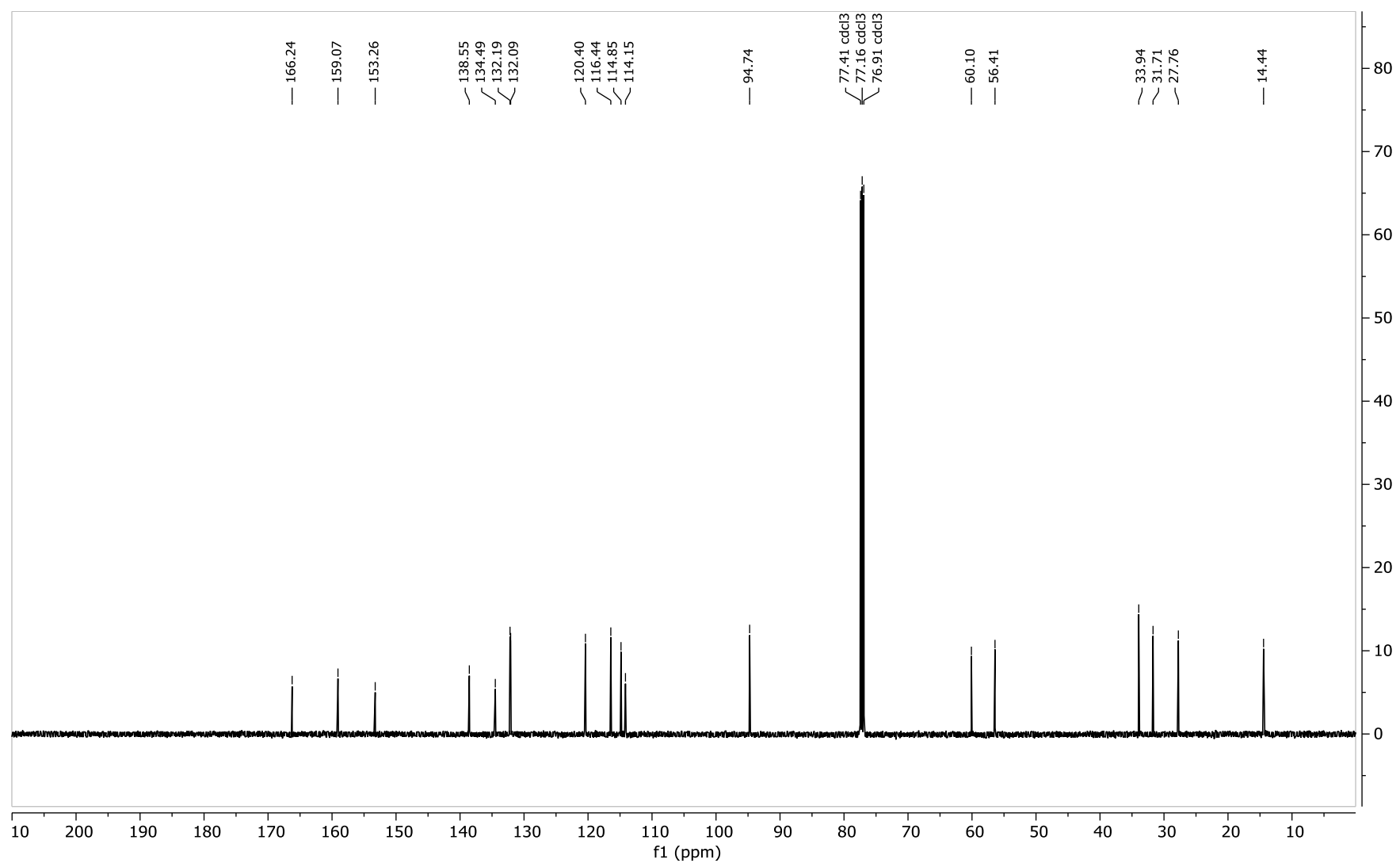




\section{S-9}

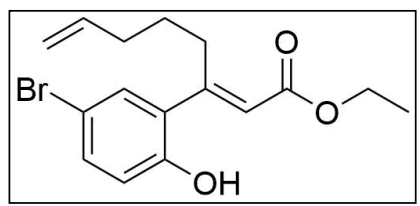

${ }^{1} \mathrm{H}$ NMR $\left(500 \mathrm{MHz}, \mathrm{CDCl}_{3}\right)$

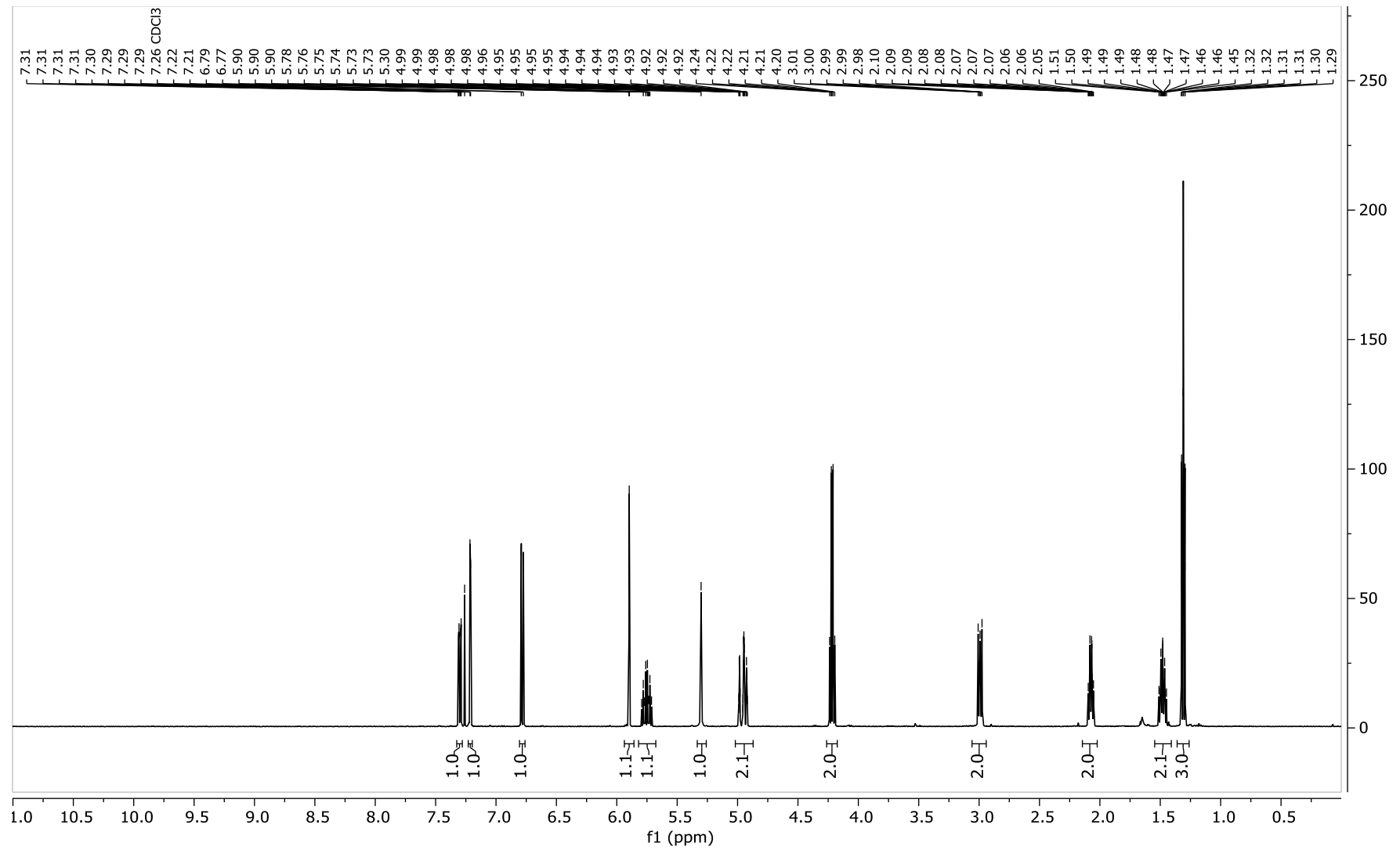

${ }^{13} \mathrm{C}$ NMR (126 MHz, $\mathrm{CDCl}_{3}$ )

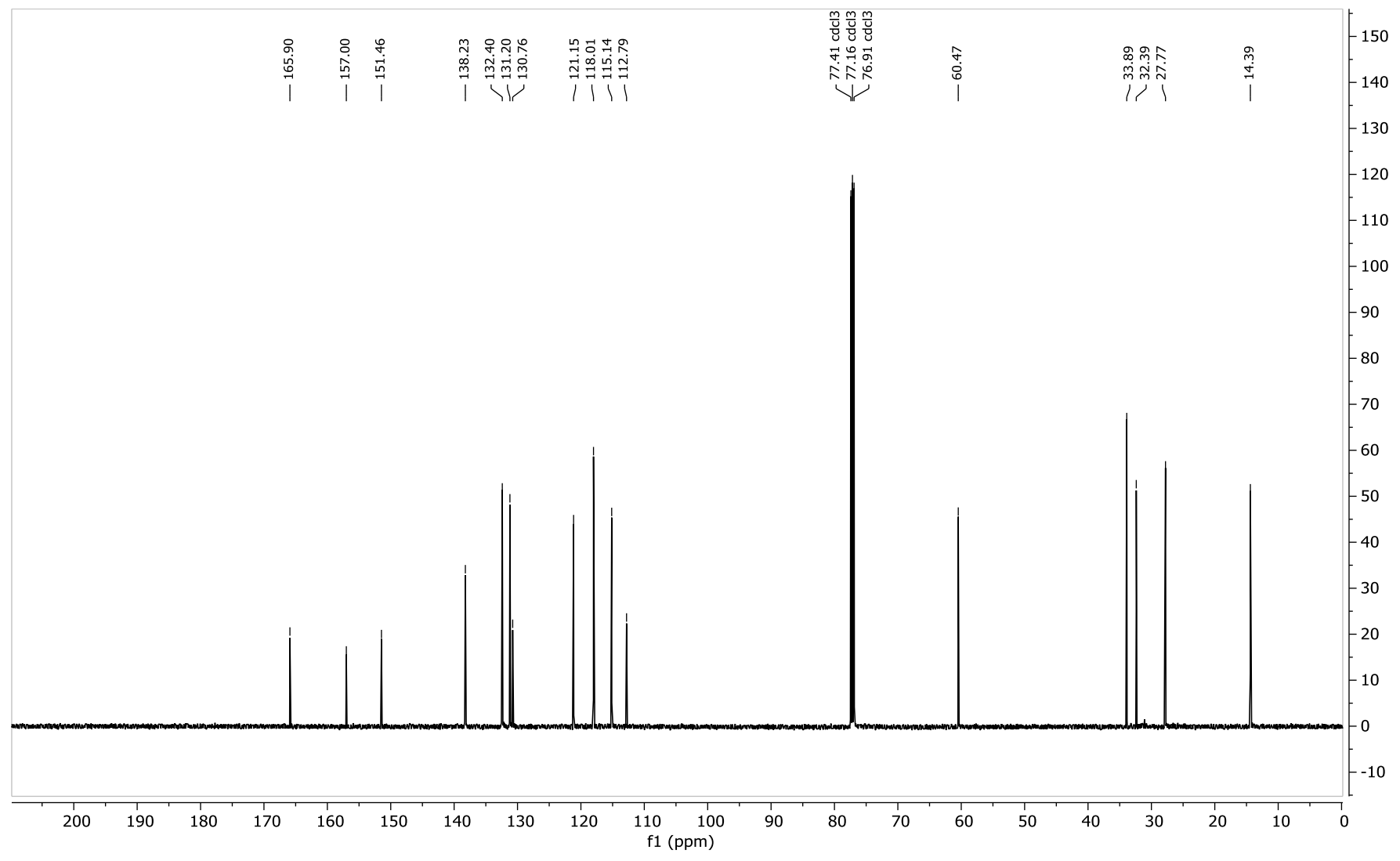



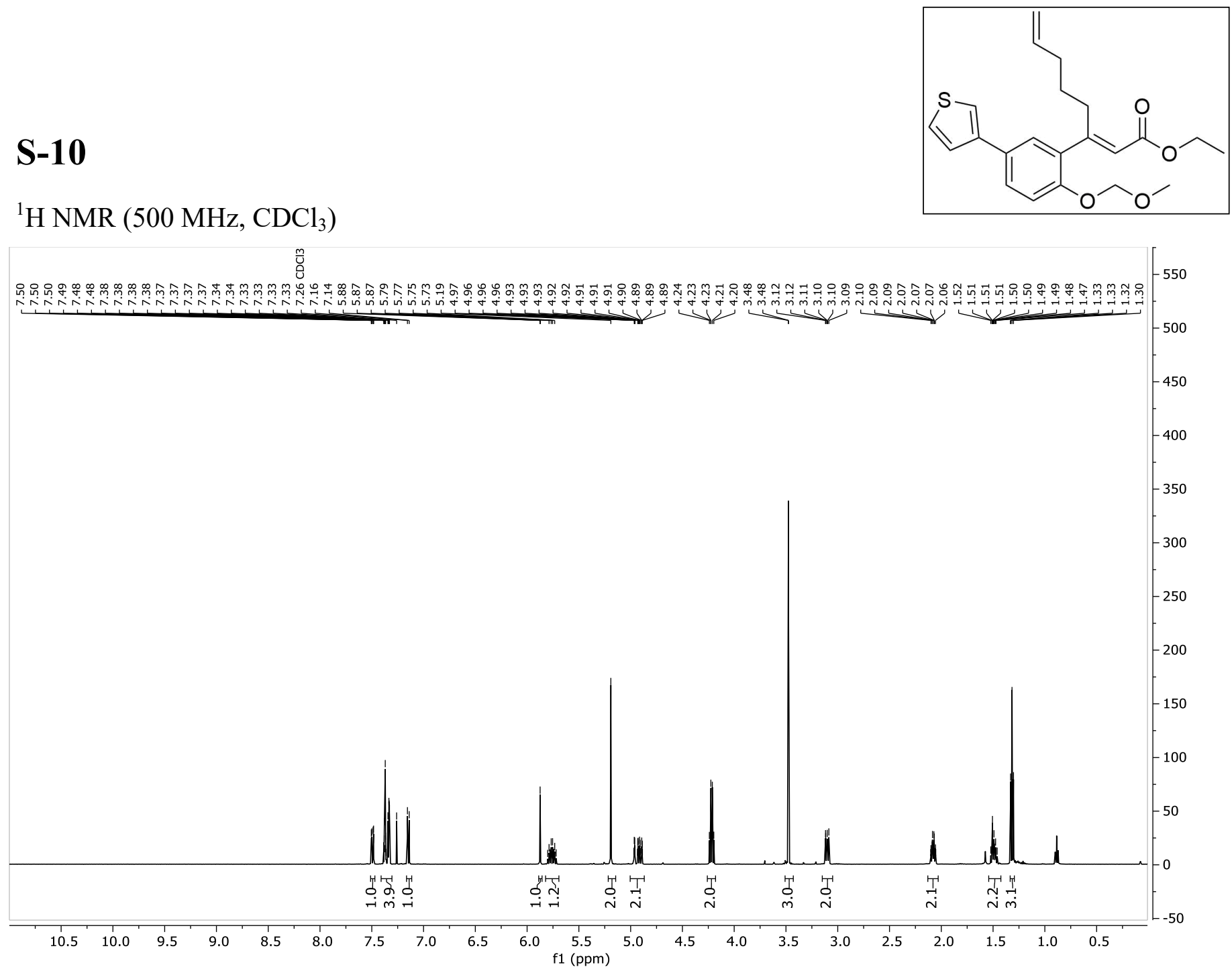

${ }^{13} \mathrm{C}$ NMR (126 MHz, $\mathrm{CDCl}_{3}$ )

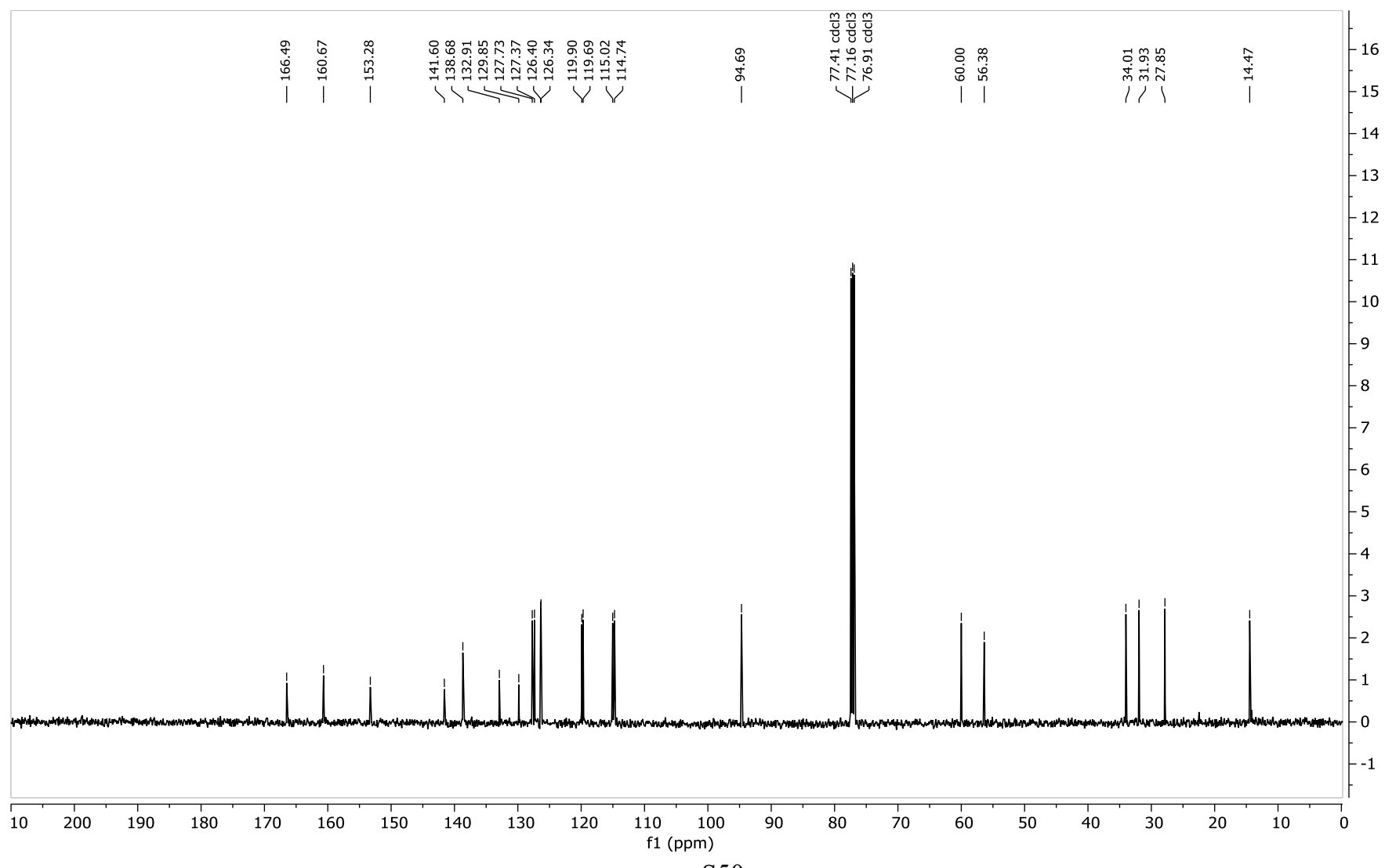


${ }^{1} \mathrm{H}$ NMR $\left(500 \mathrm{MHz}, \mathrm{CDCl}_{3}\right)$

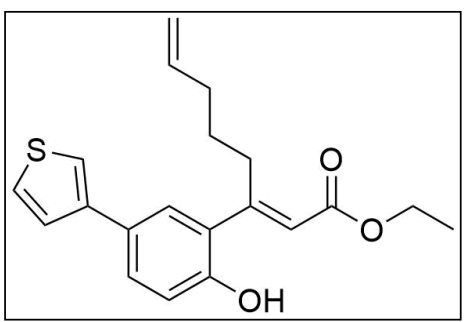

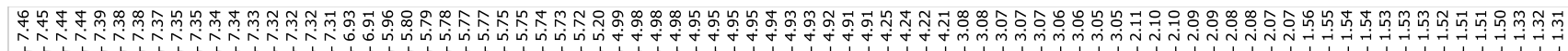

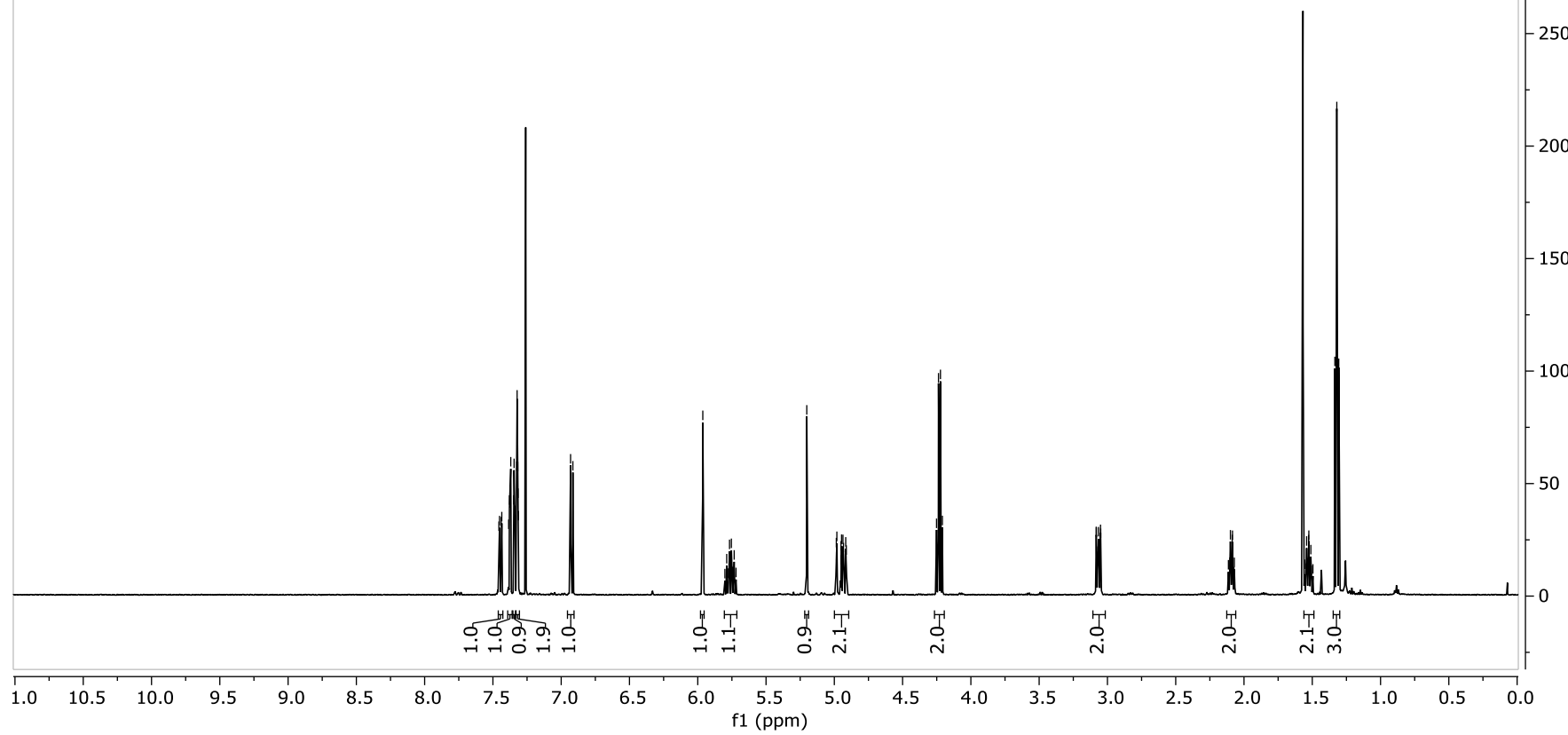

${ }^{13} \mathrm{C}$ NMR $\left(126 \mathrm{MHz}, \mathrm{CDCl}_{3}\right)$

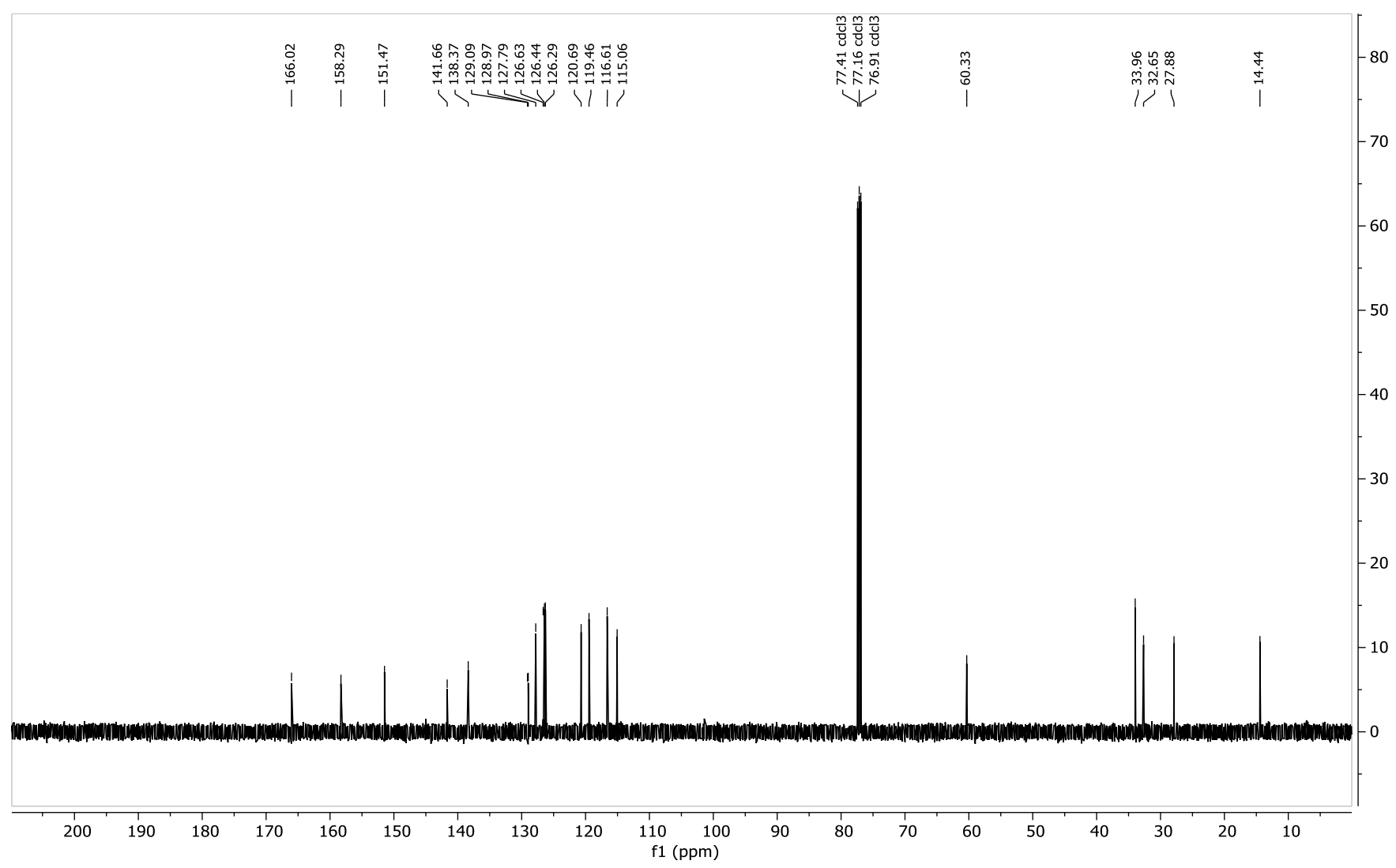




\section{S-12}

${ }^{1} \mathrm{H}$ NMR $\left(500 \mathrm{MHz}, \mathrm{CDCl}_{3}\right)$
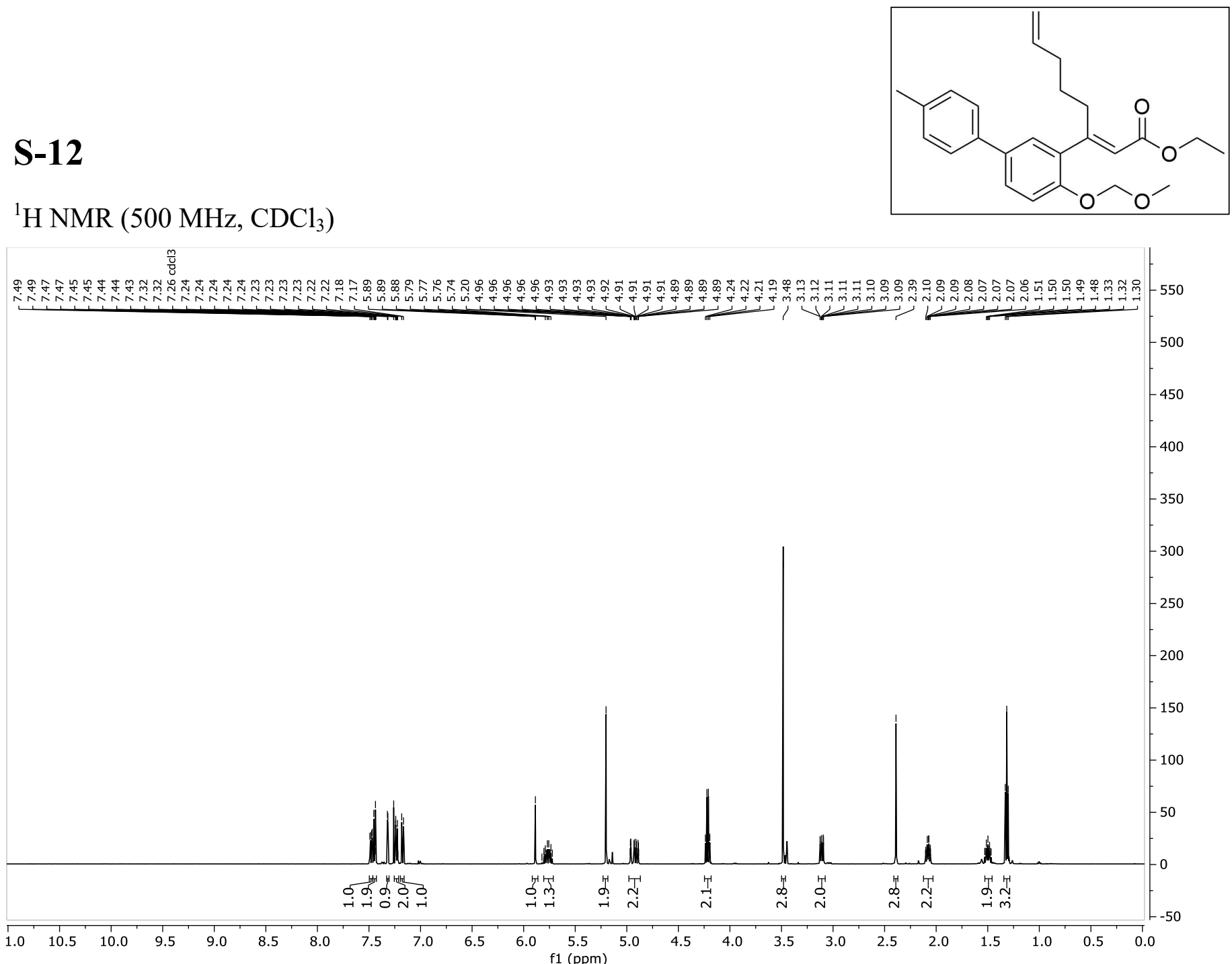

${ }^{13} \mathrm{C}$ NMR $\left(126 \mathrm{MHz}, \mathrm{CDCl}_{3}\right)$

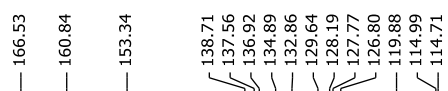

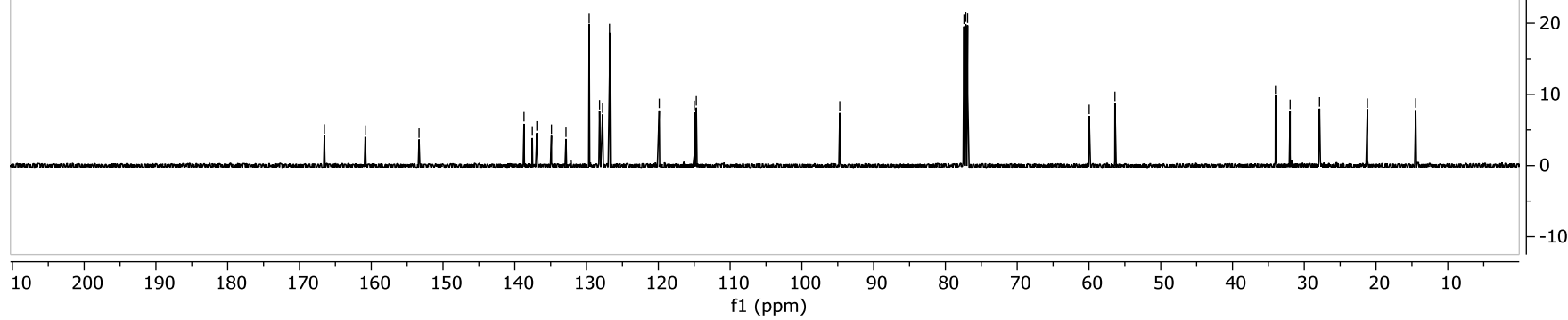




\section{S-13}

${ }^{1} \mathrm{H}$ NMR (599 MHz, $\mathrm{CDCl}_{3}$ )
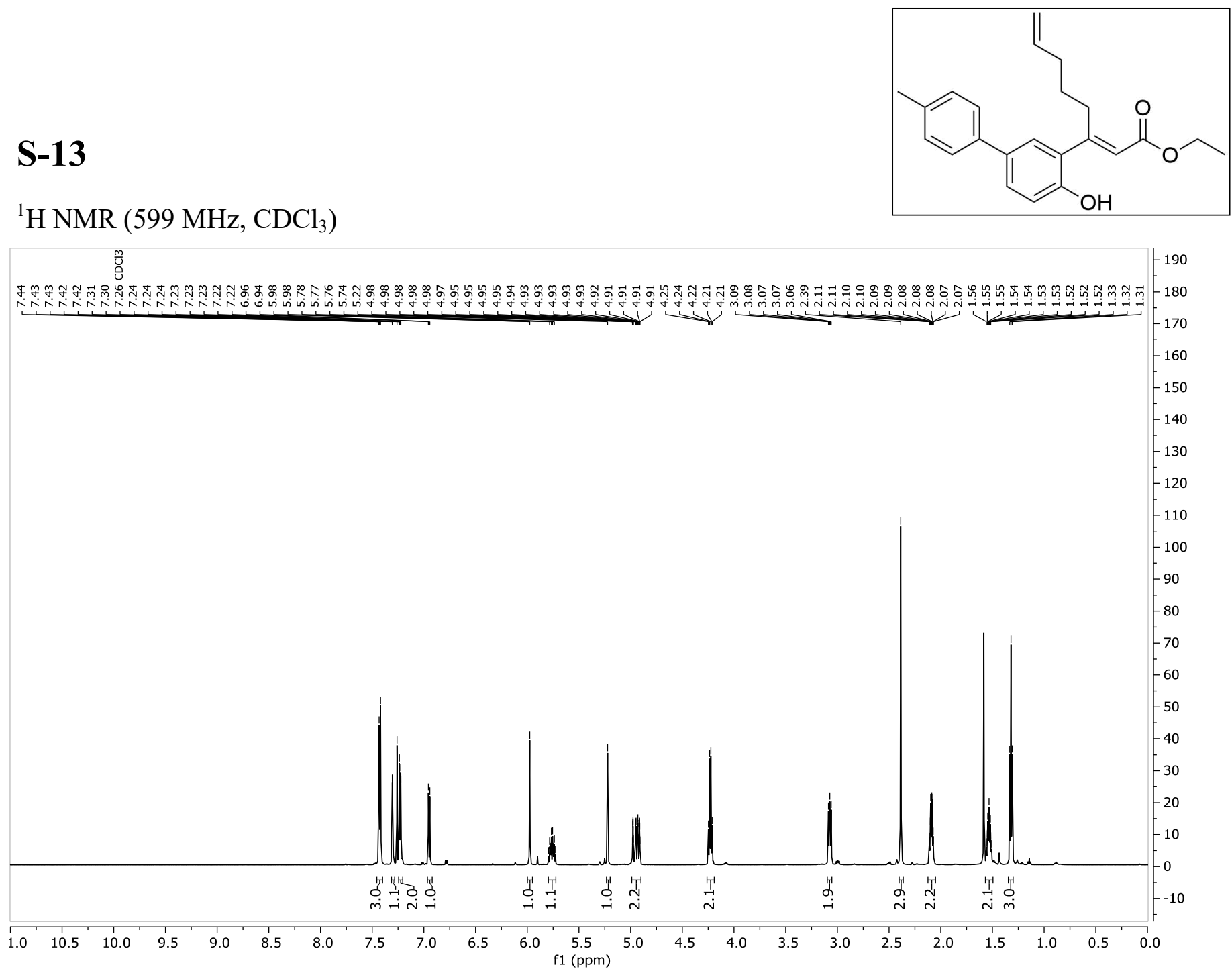

${ }^{13} \mathrm{C}$ NMR (151 MHz, $\mathrm{CDCl}_{3}$ )

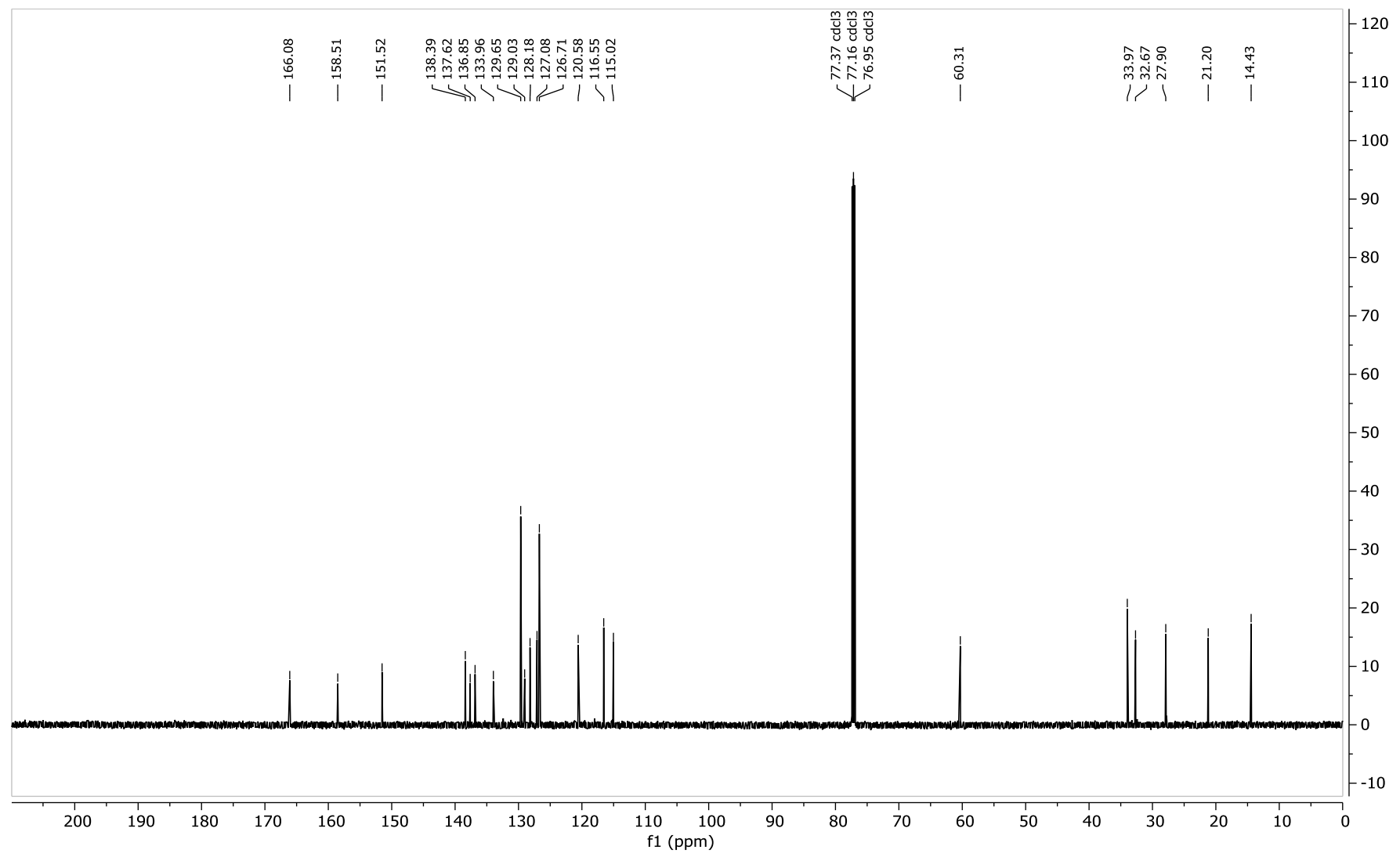




\section{S-14}

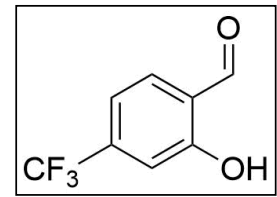

${ }^{1} \mathrm{H}$ NMR $\left(400 \mathrm{MHz}, \mathrm{CDCl}_{3}\right)$

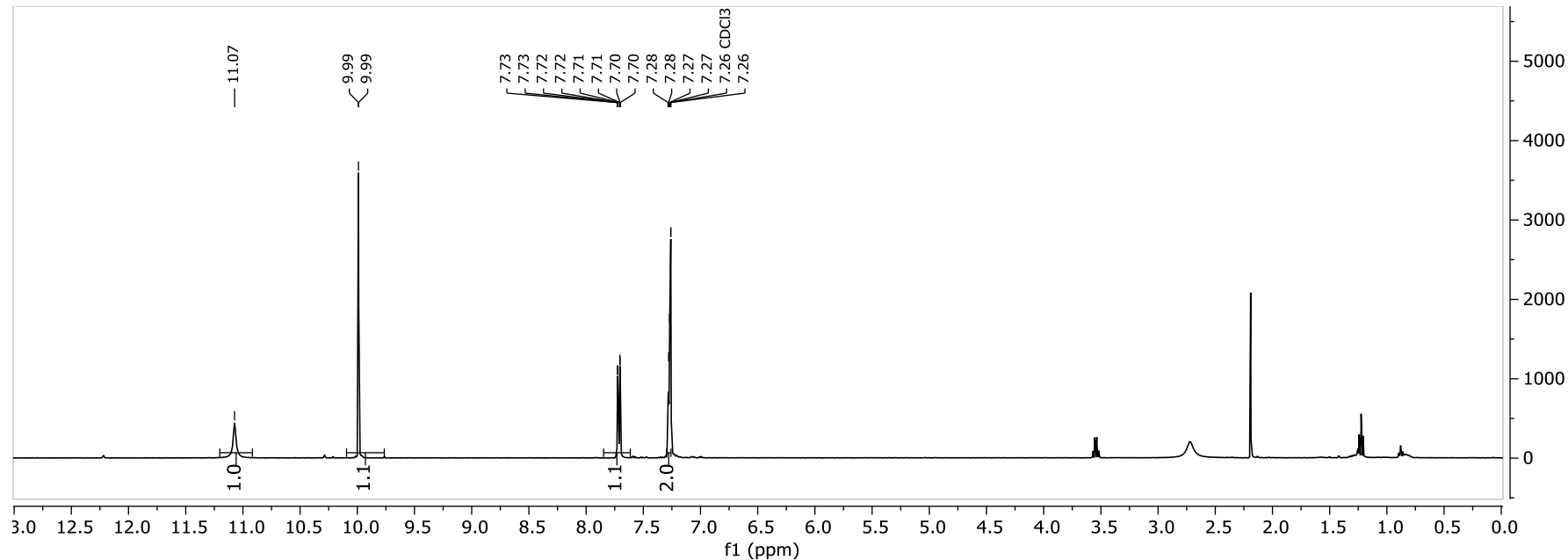

${ }^{13} \mathrm{C}$ NMR $\left(101 \mathrm{MHz}, \mathrm{CDCl}_{3}\right)$

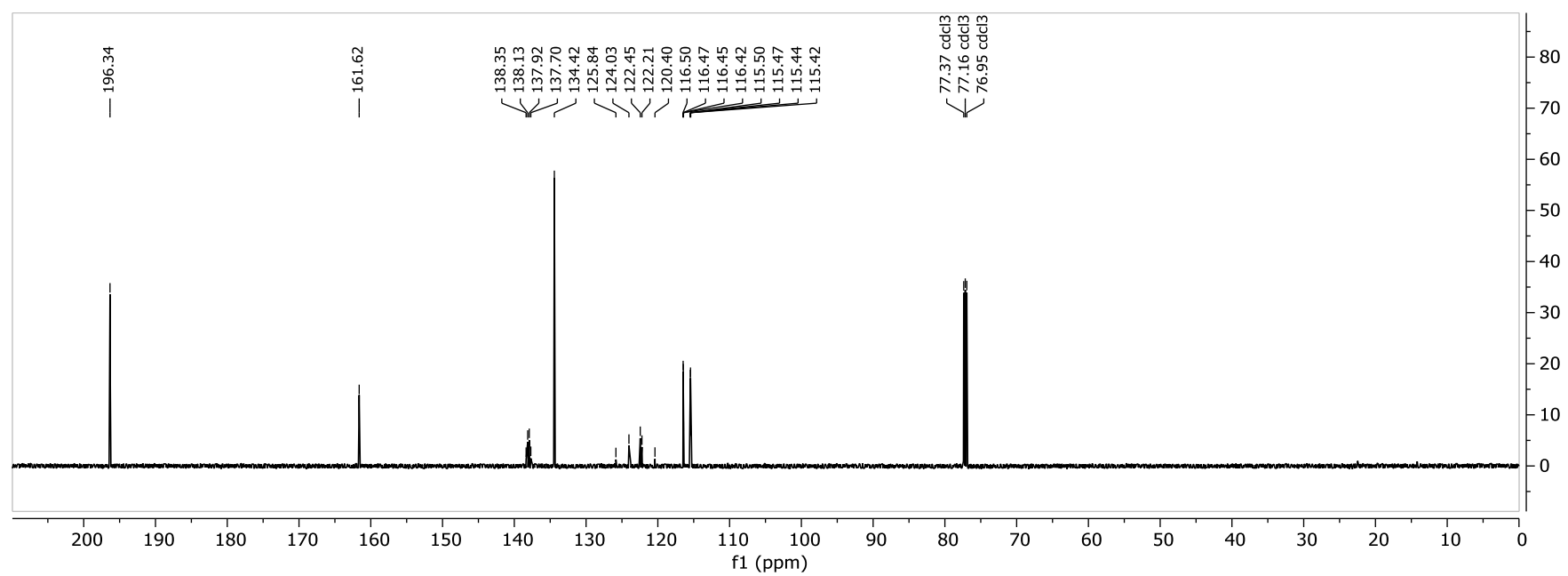

${ }^{19} \mathrm{~F}$ NMR $(376 \mathrm{MHz}$

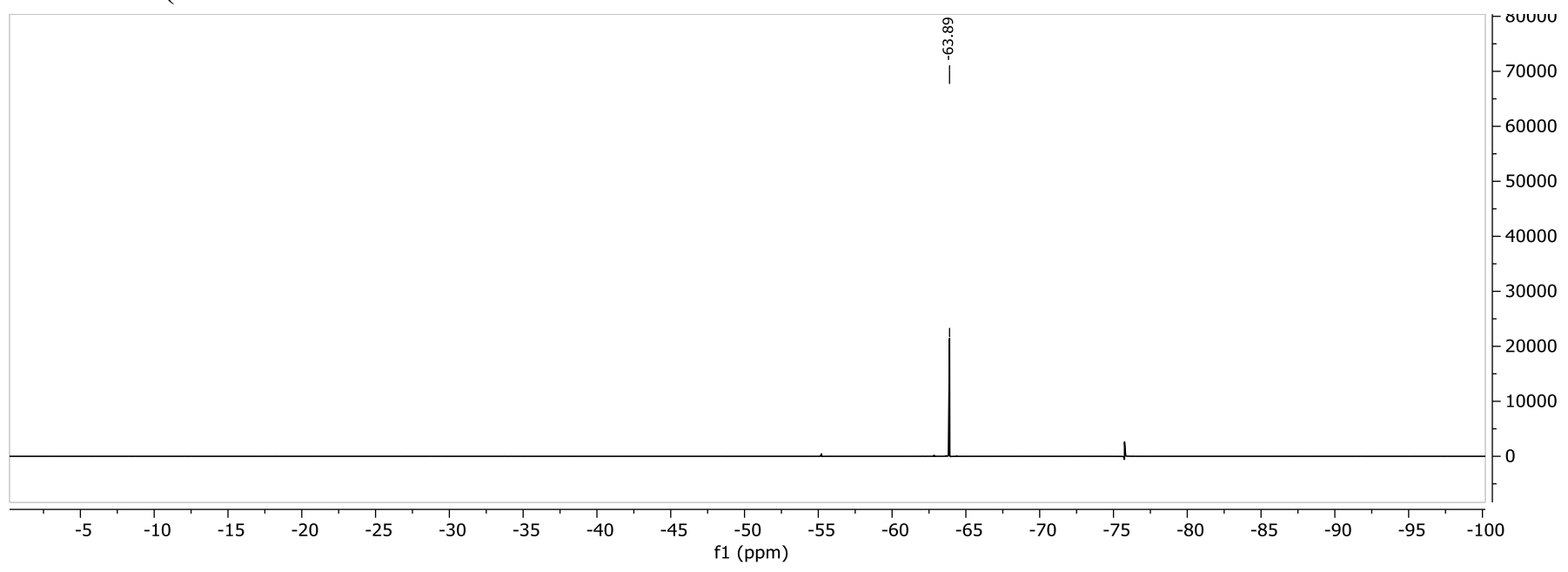




\section{S-15}

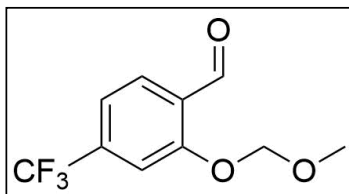

${ }^{1} \mathrm{H}$ NMR $\left(400 \mathrm{MHz}, \mathrm{CDCl}_{3}\right)$

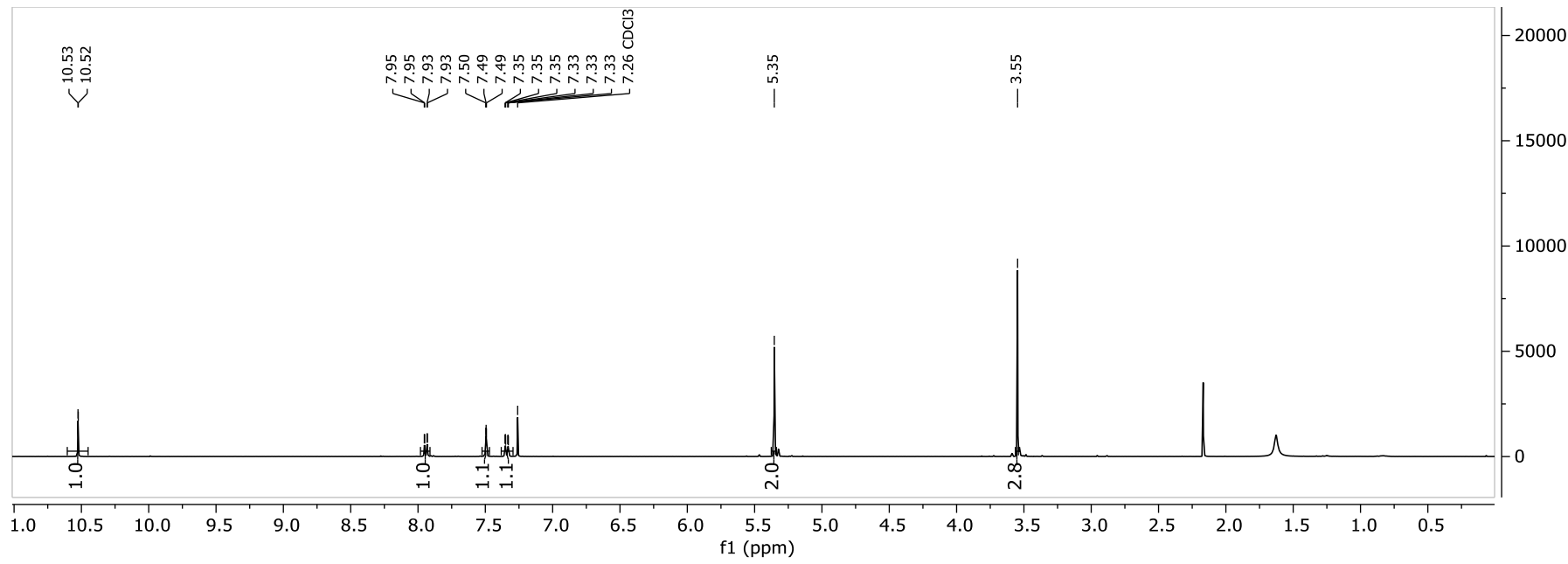

${ }^{13} \mathrm{C}$ NMR $\left(151 \mathrm{MHz}, \mathrm{CDCl}_{3}\right)$

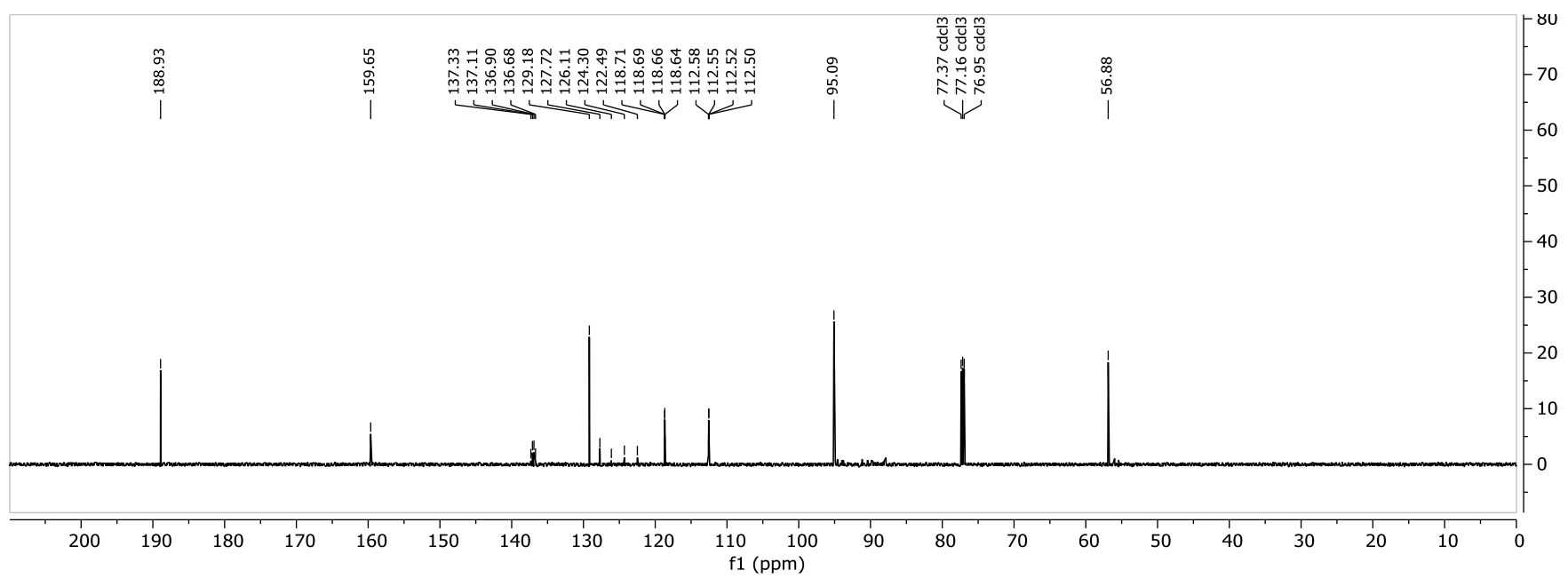

${ }^{19} \mathrm{~F}$ NMR $\left(376 \mathrm{MHz}, \mathrm{CDCL}_{3}\right)$

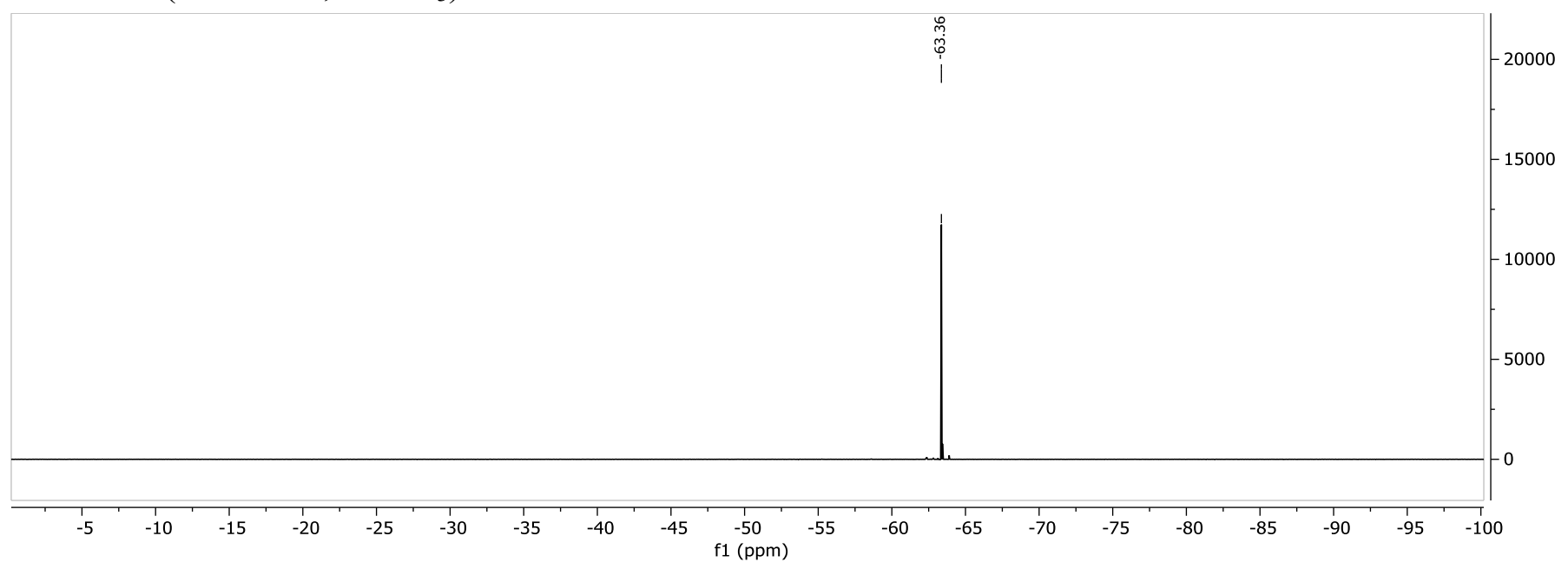




\section{S-16}

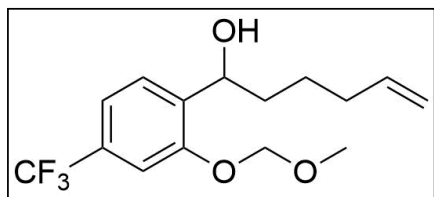

${ }^{1} \mathrm{H}$ NMR (599 MHz, $\mathrm{CDCl}_{3}$ )

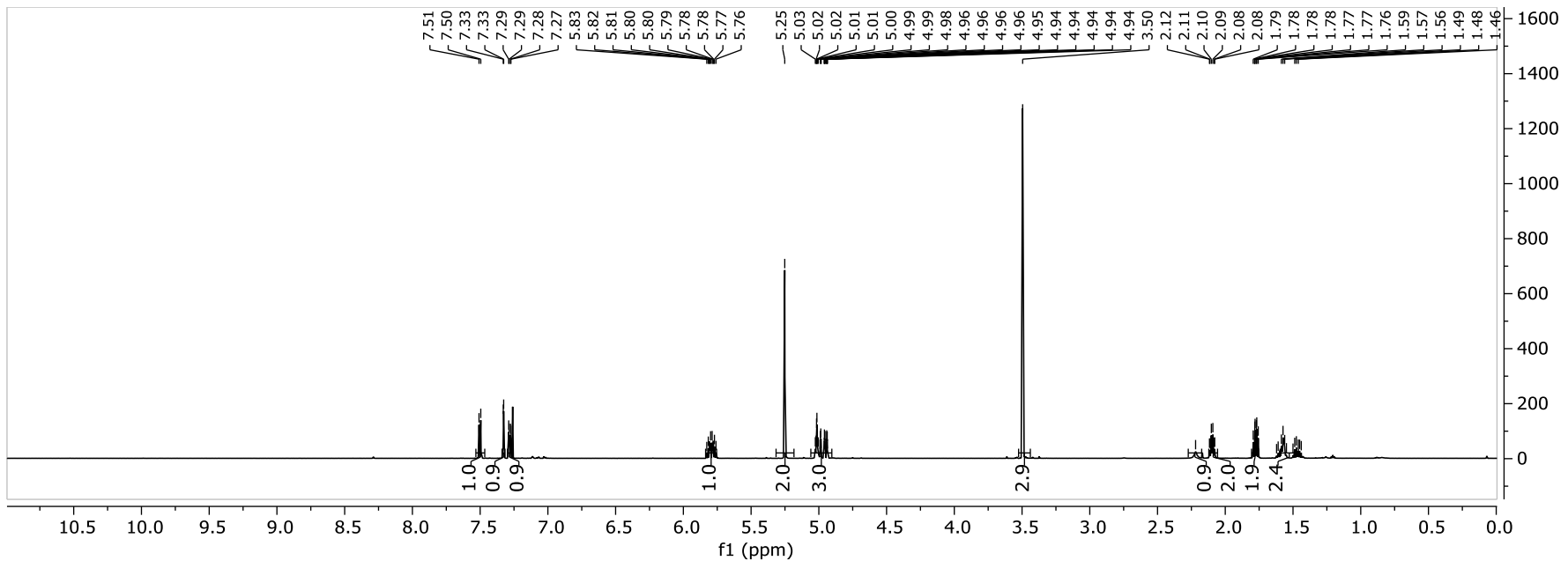

${ }^{13} \mathrm{C}$ NMR $\left(151 \mathrm{MHz}, \mathrm{CDCl}_{3}\right)$

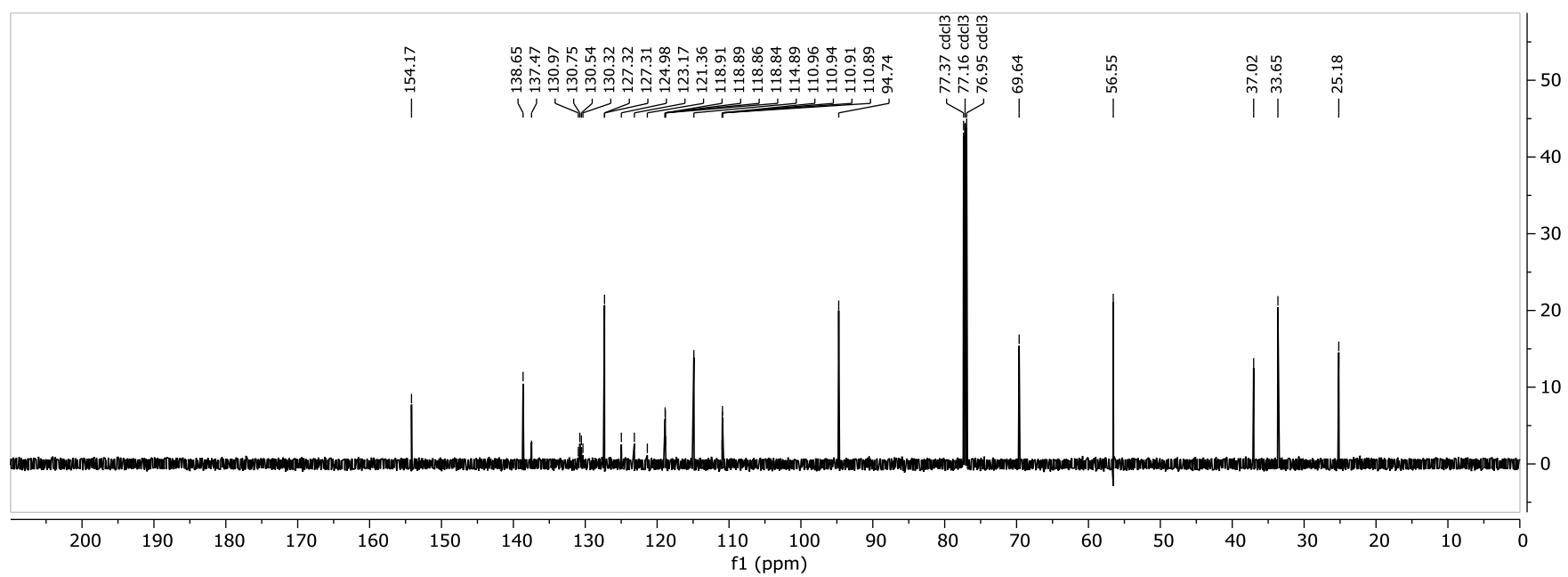

${ }^{19}$ F NMR (564 MHz, $\left.\mathrm{CDCl}_{3}\right)$

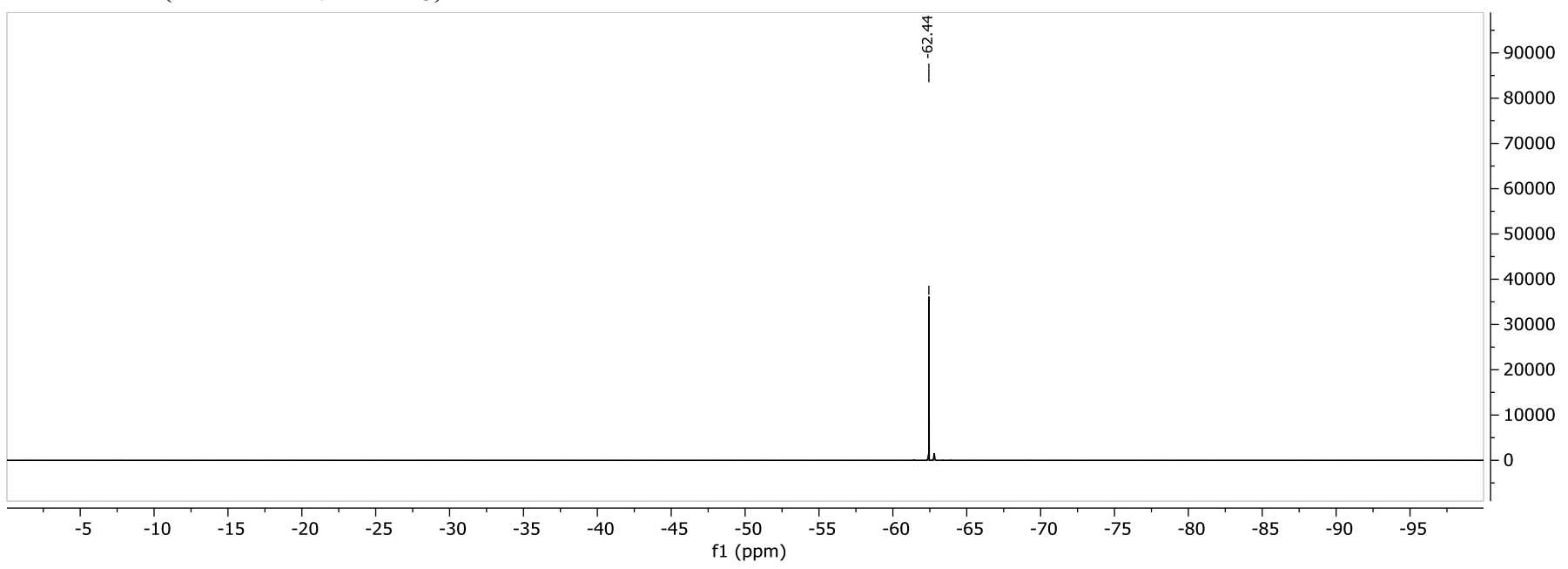




\section{S-17}

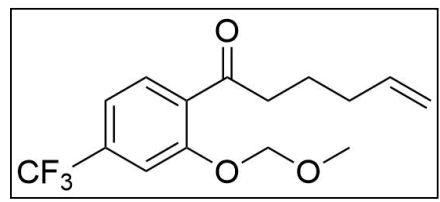

${ }^{1} \mathrm{H}$ NMR $\left(599 \mathrm{MHz}, \mathrm{CDCl}_{3}\right.$ )

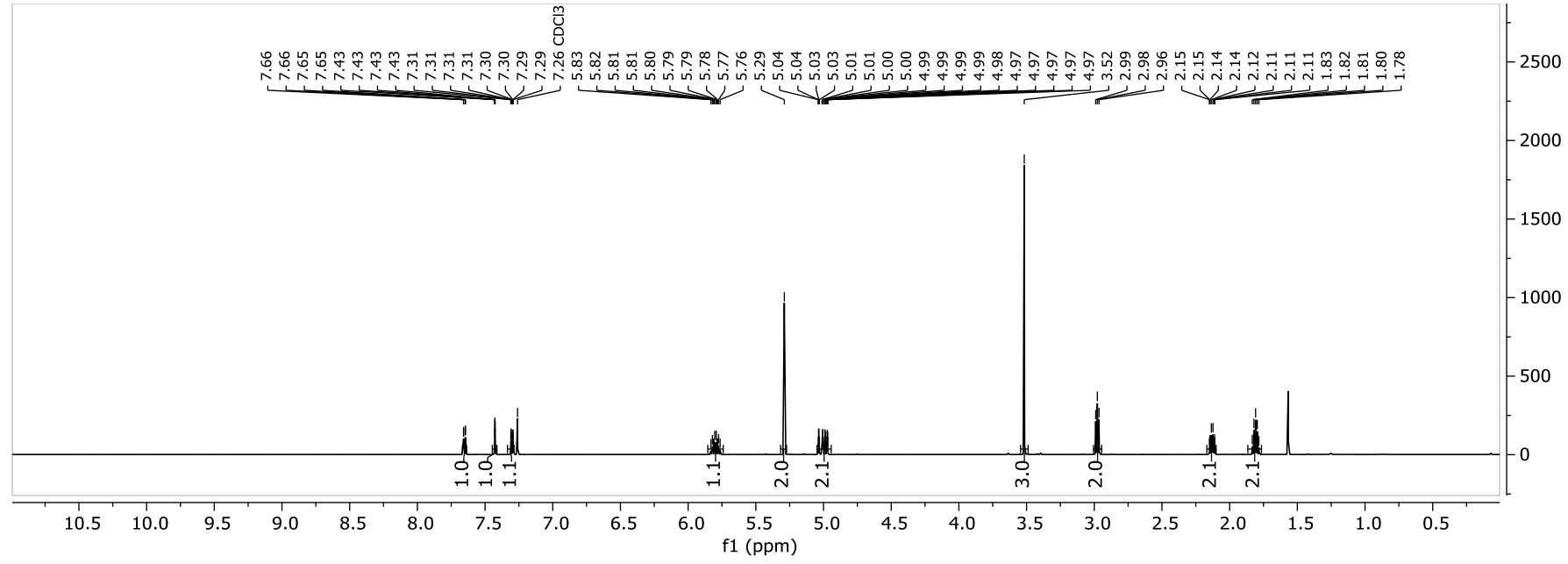

${ }^{13} \mathrm{C}$ NMR $\left(151 \mathrm{MHz}, \mathrm{CDCl}_{3}\right)$

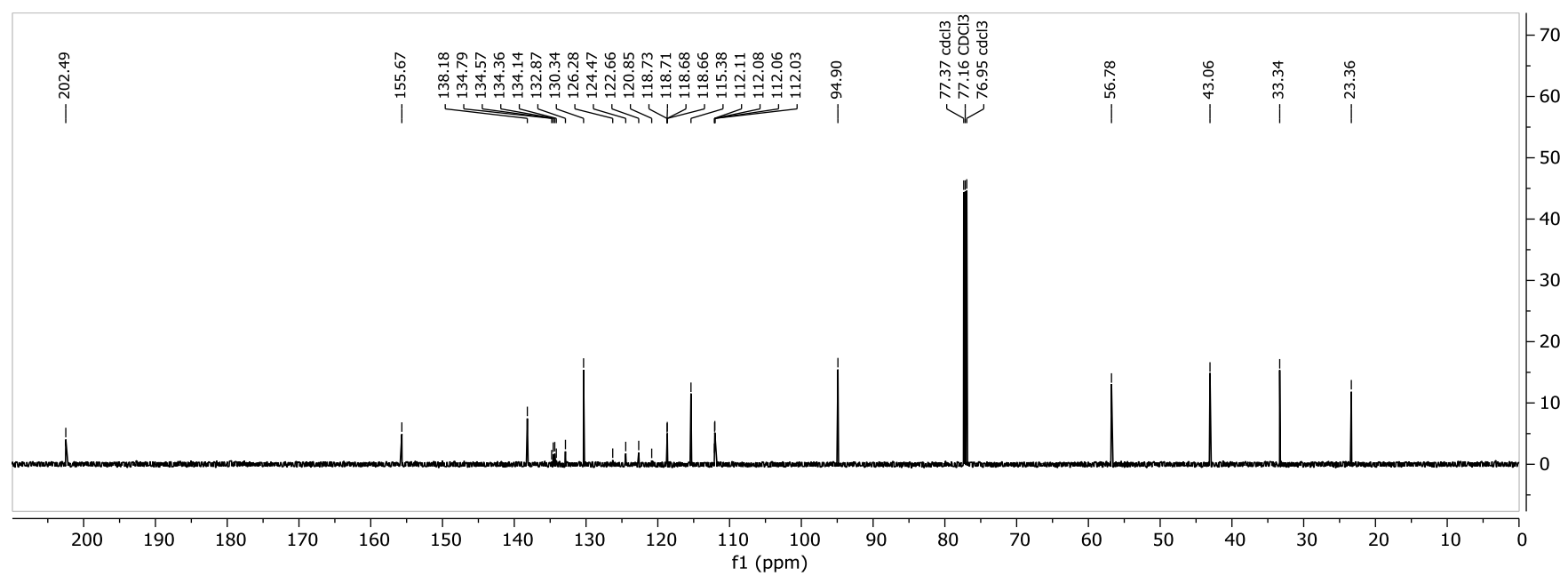

${ }^{19} \mathrm{~F}$ NMR $\left(564 \mathrm{MHz}, \mathrm{CDCl}_{3}\right)$

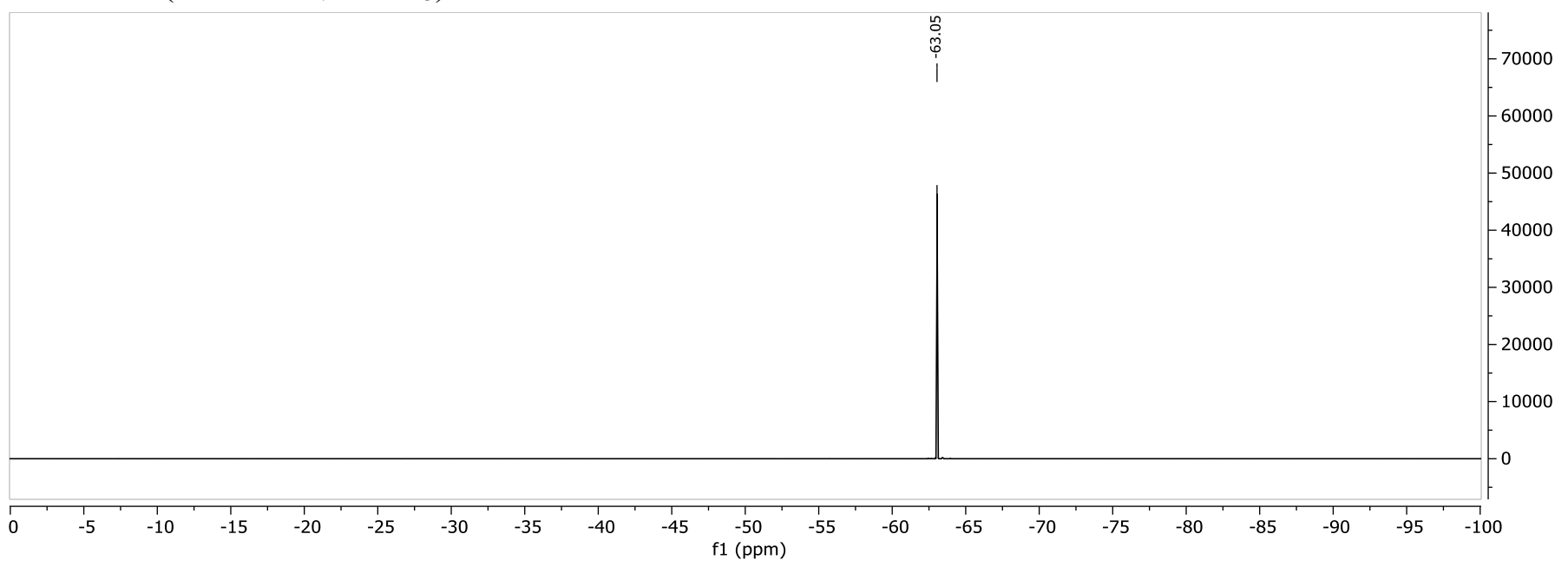




\section{S-18}

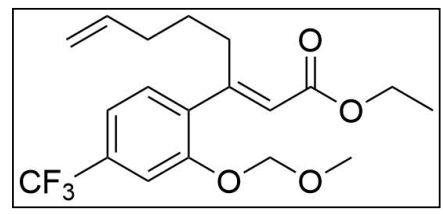

${ }^{1} \mathrm{H}$ NMR $\left(500 \mathrm{MHz}, \mathrm{CDCl}_{3}\right)$

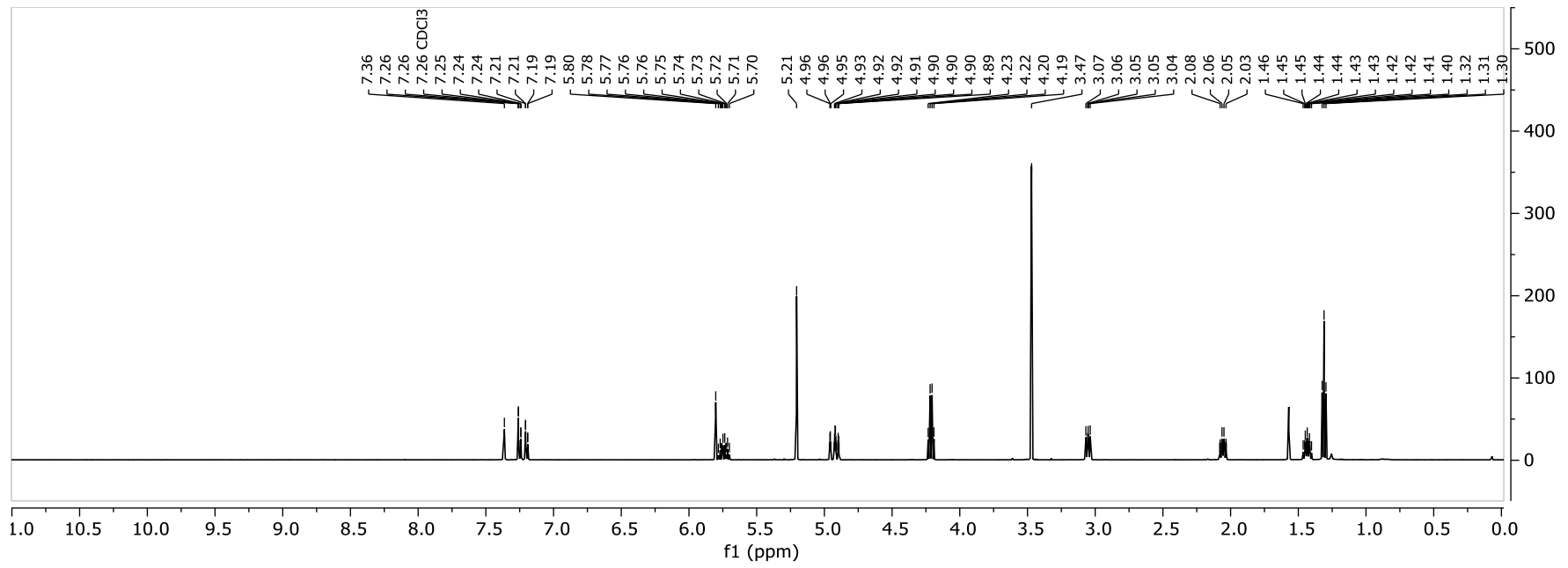

${ }^{13} \mathrm{C}$ NMR (126 MHz, $\mathrm{CDCl}_{3}$ )

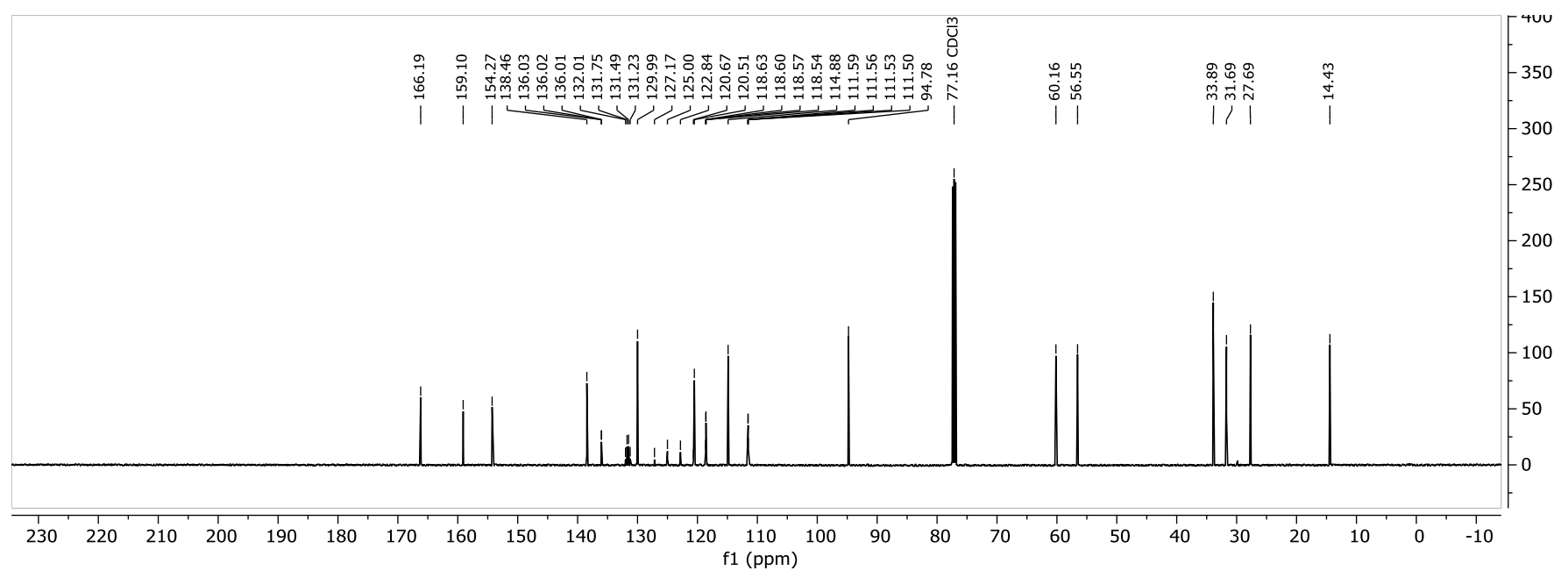

${ }^{19}$ F NMR $(376 \mathrm{MHz}$

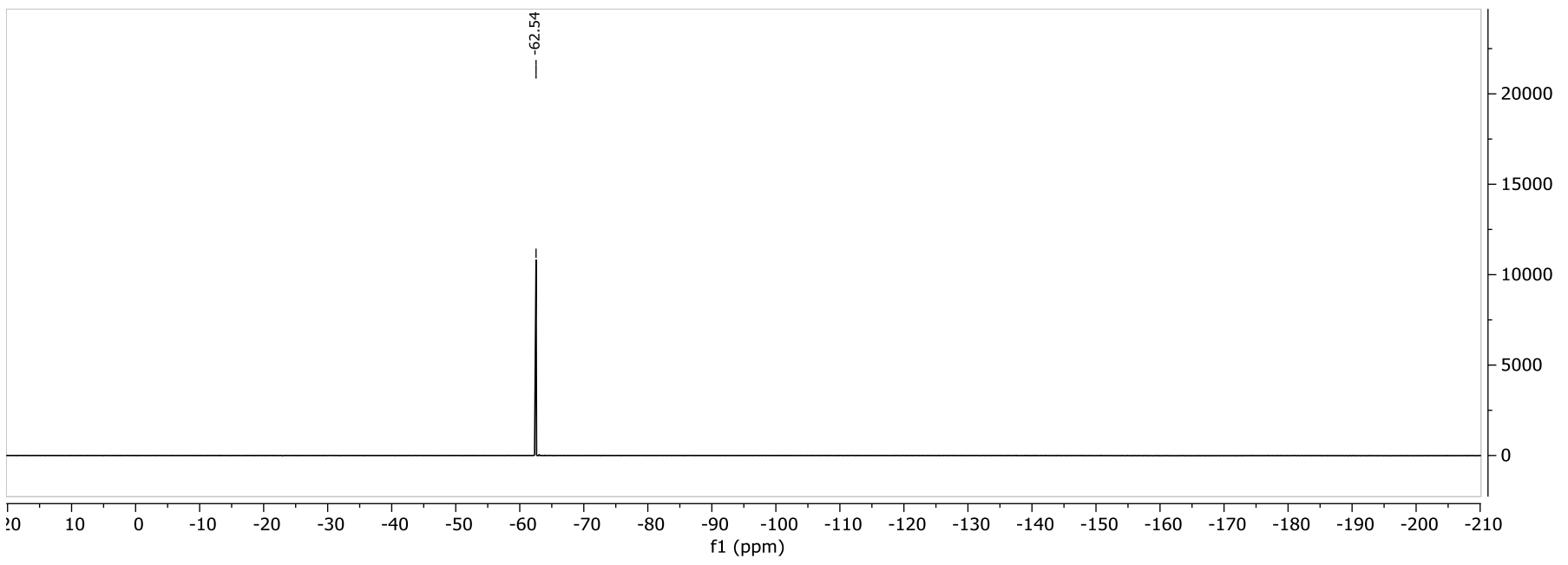




\section{S-19}

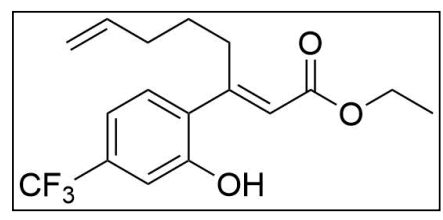

${ }^{1} \mathrm{H}$ NMR $\left(500 \mathrm{MHz}, \mathrm{CDCl}_{3}\right)$

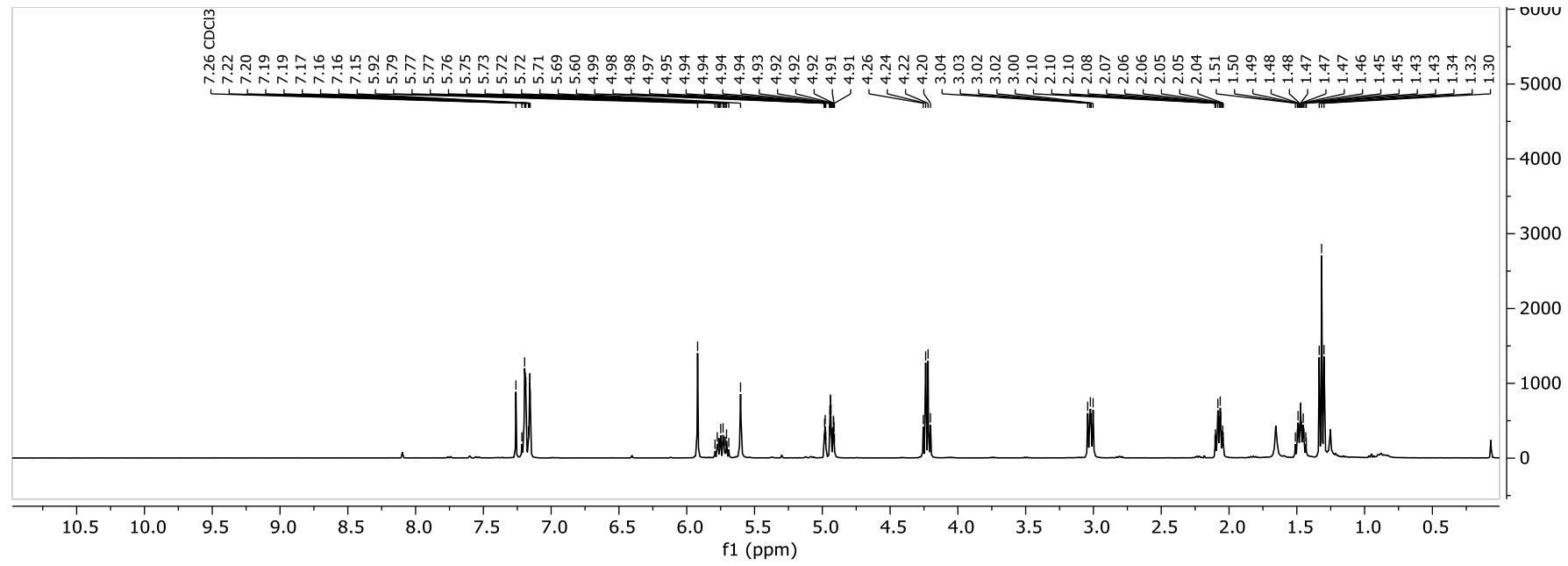

${ }^{13} \mathrm{C}$ NMR $\left(126 \mathrm{MHz}, \mathrm{CDCl}_{3}\right)$

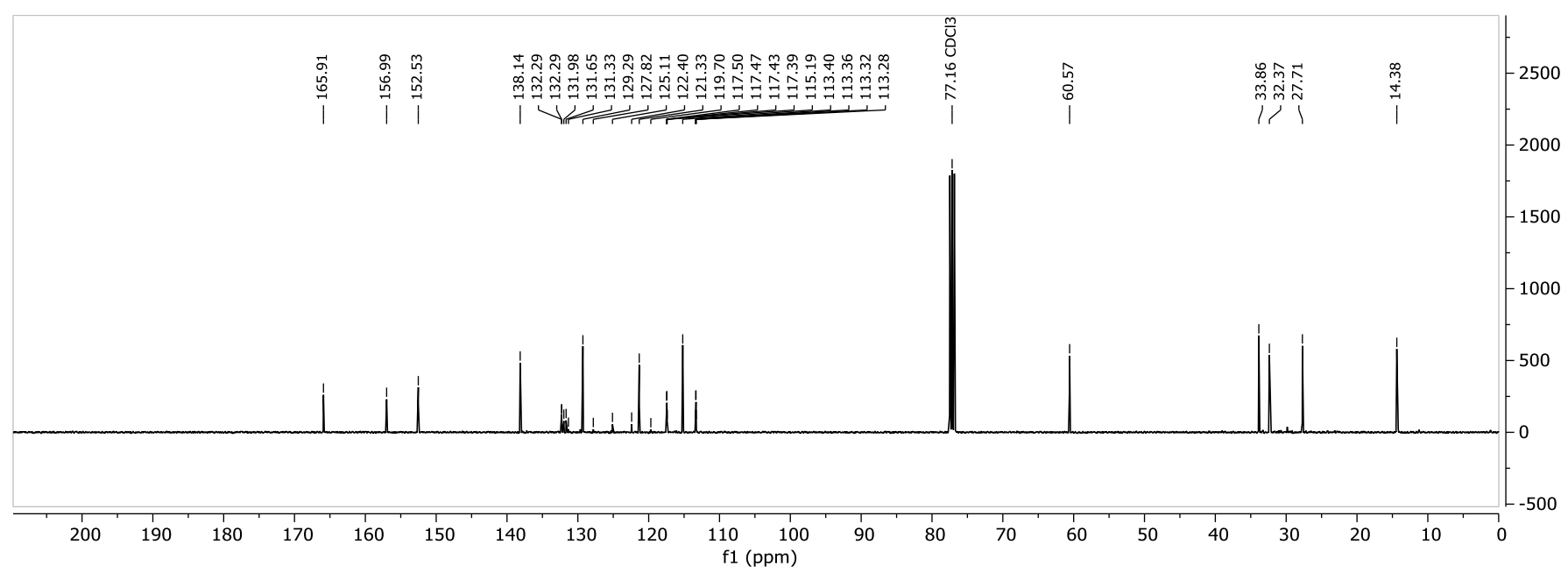

${ }^{19} \mathrm{~F}$ NMR $(376 \mathrm{MHz}$

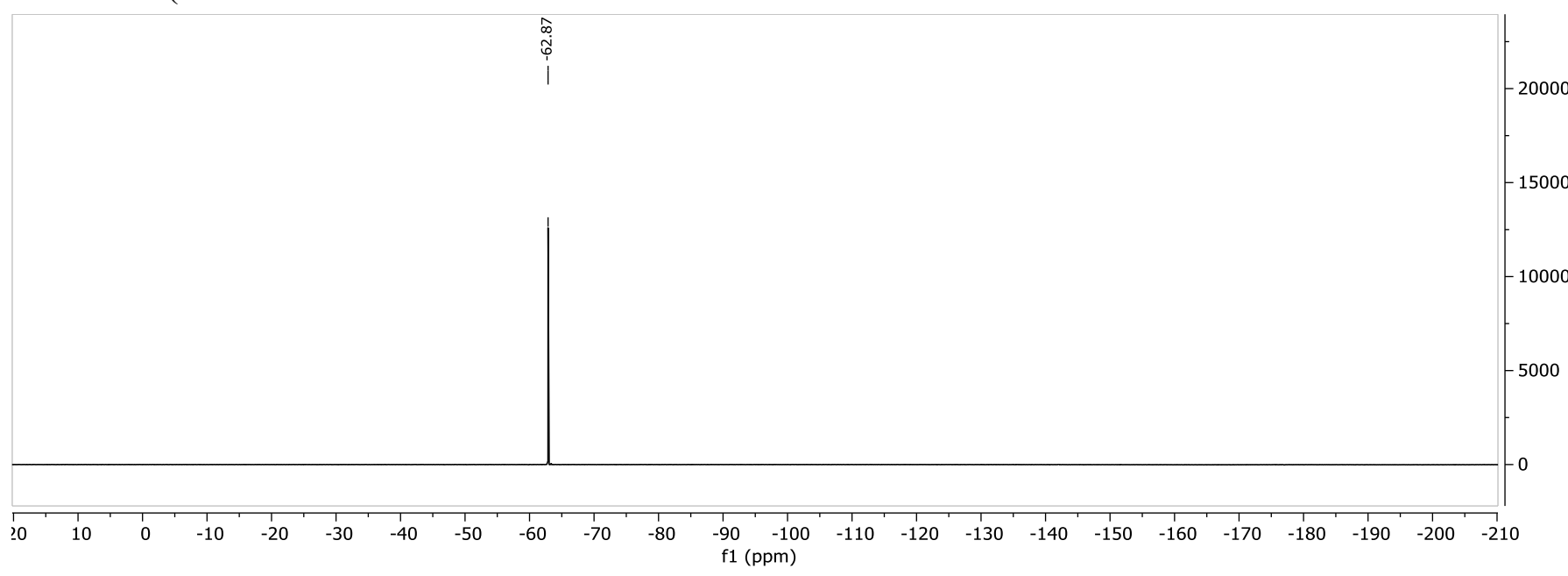


${ }^{1} \mathrm{H}$ NMR $\left(500 \mathrm{MHz}, \mathrm{CDCl}_{3}\right)$

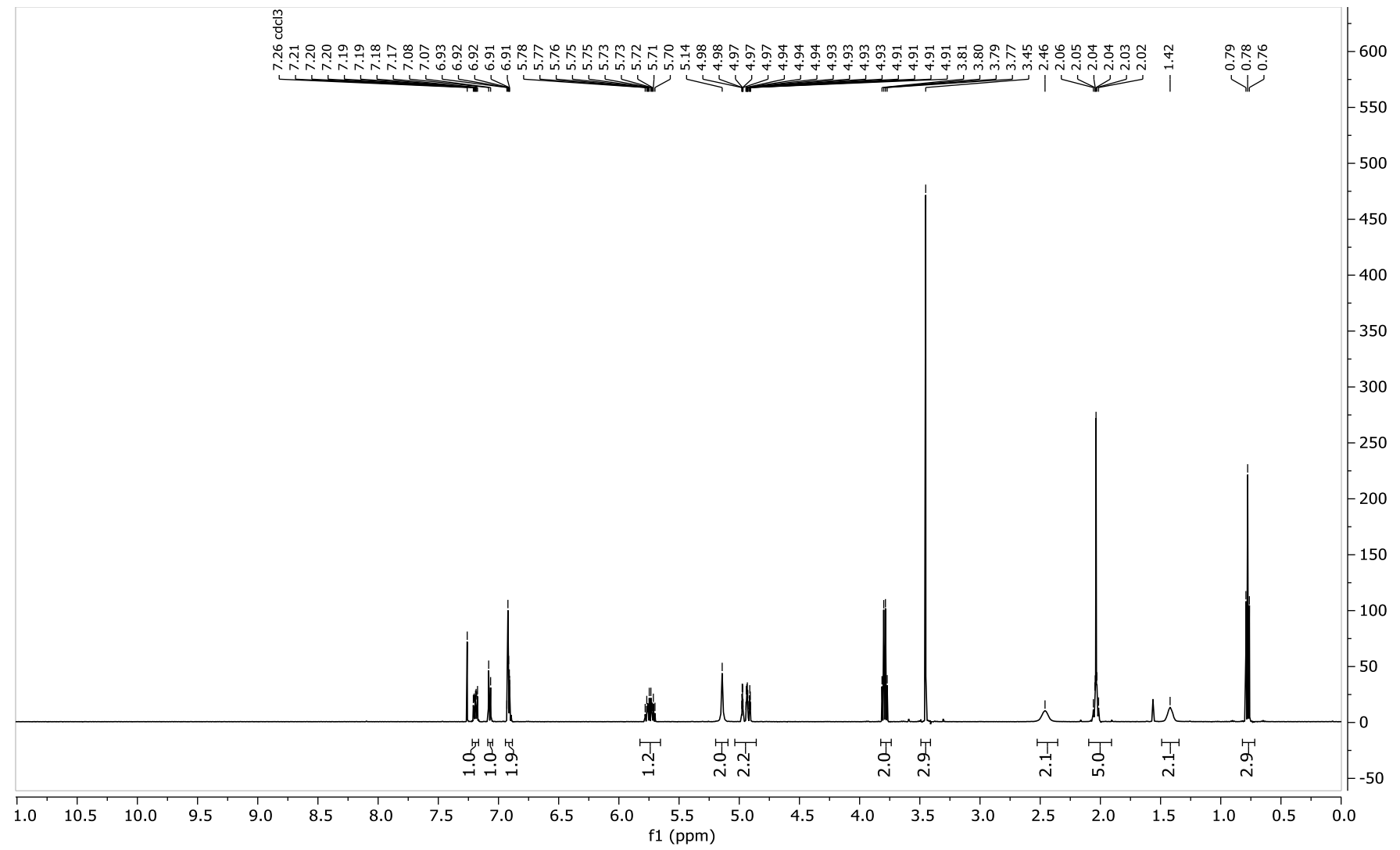

${ }^{13} \mathrm{C}$ NMR (126 MHz, $\mathrm{CDCl}_{3}$ )

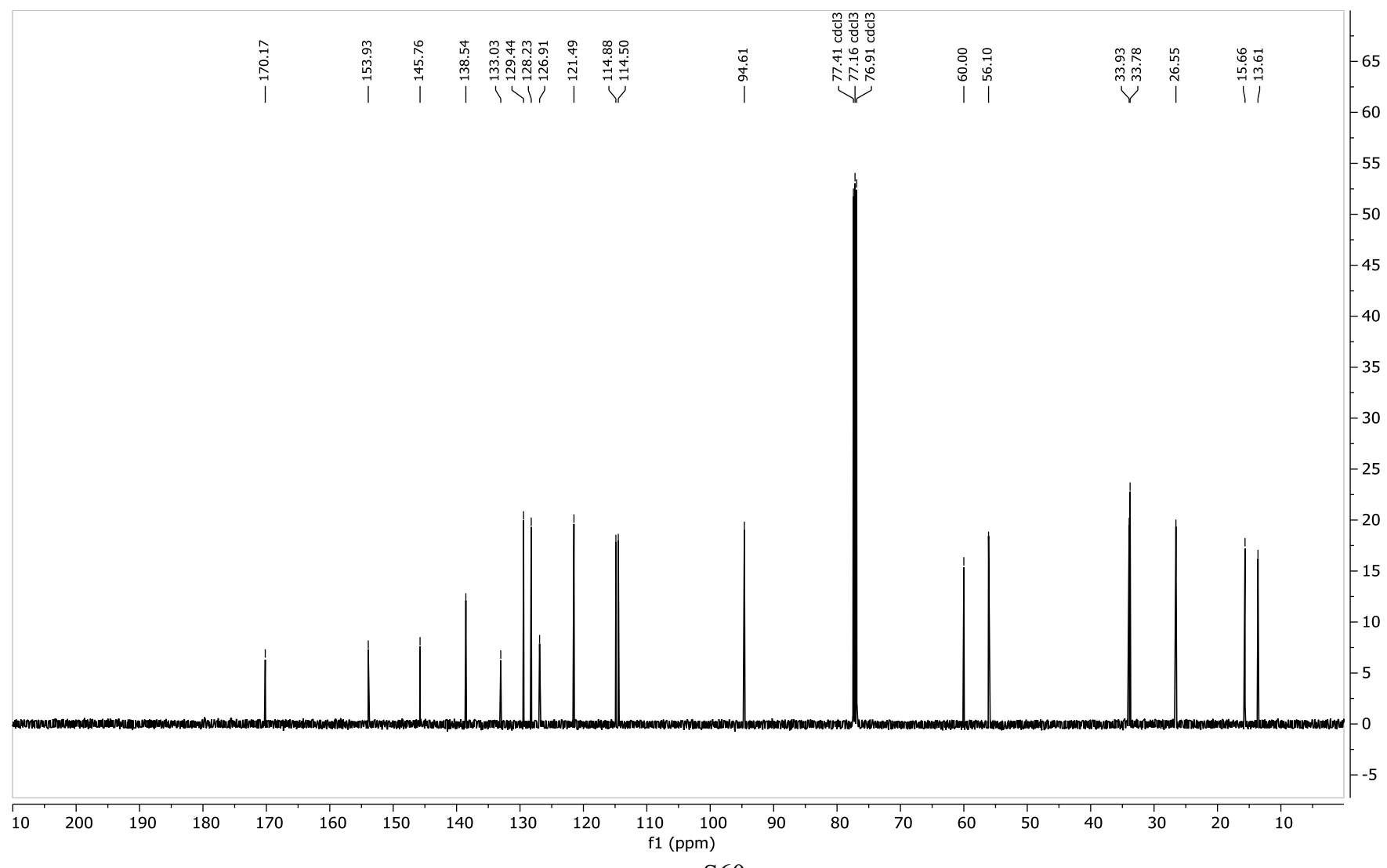




\section{S-21}

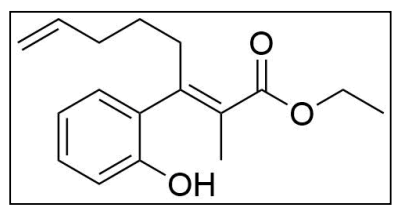

${ }^{1} \mathrm{H}$ NMR $\left(500 \mathrm{MHz}, \mathrm{CDCl}_{3}\right)$

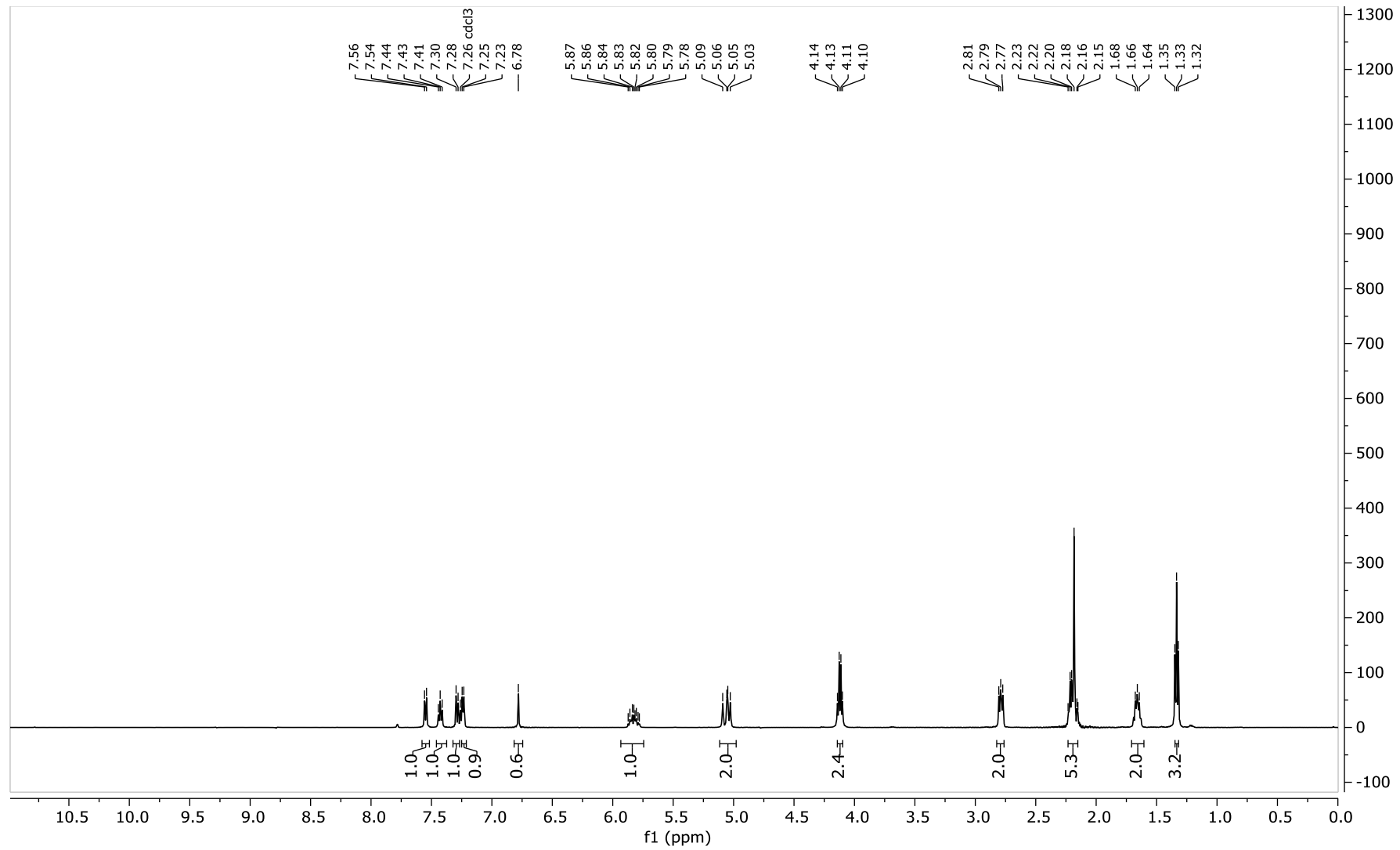

${ }^{13} \mathrm{C}$ NMR (126 MHz, $\mathrm{CDCl}_{3}$ )

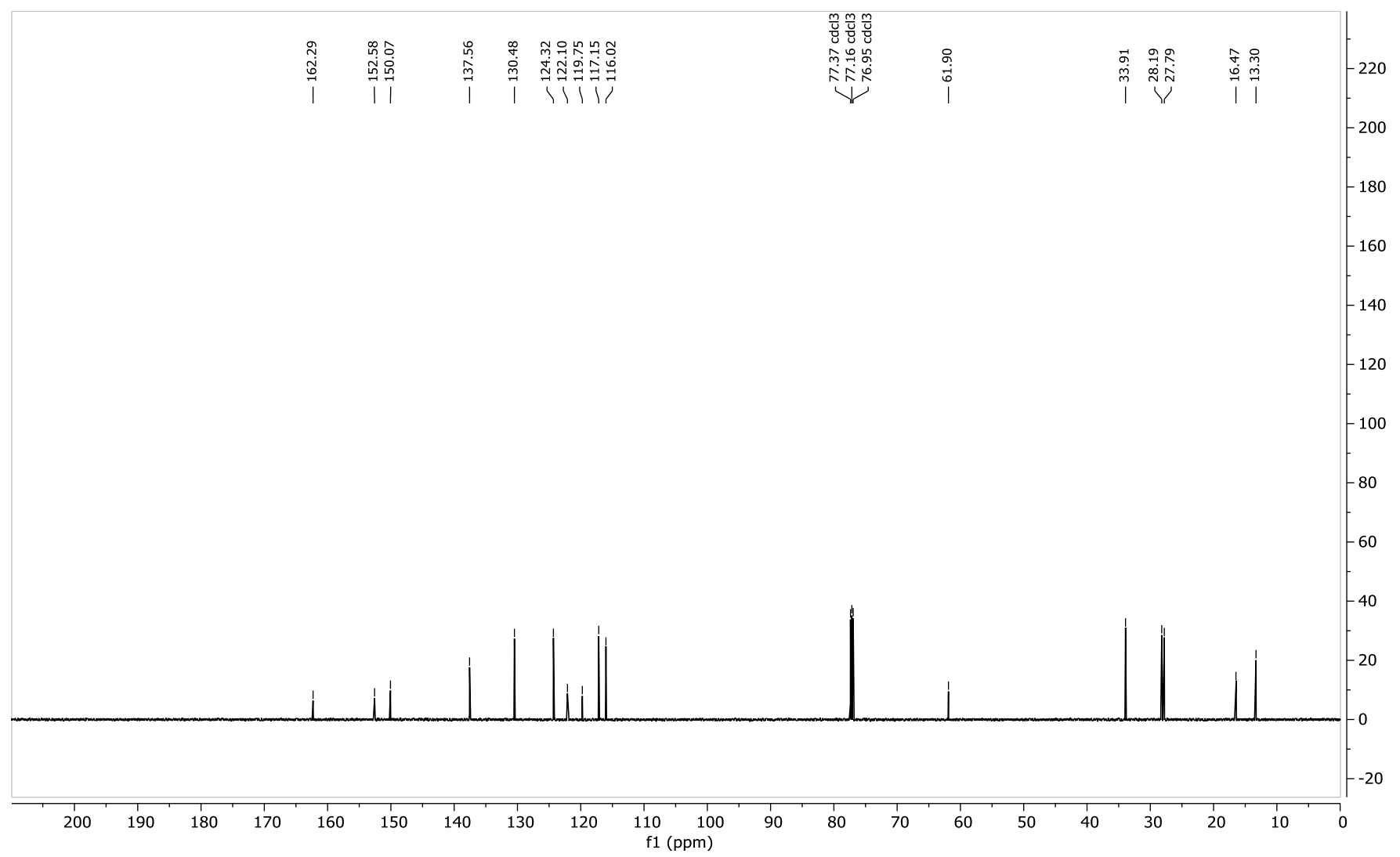




\section{S-22}

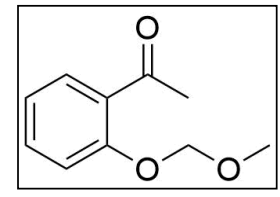

${ }^{1} \mathrm{H}$ NMR $\left(300 \mathrm{MHz}, \mathrm{CDCl}_{3}\right)$

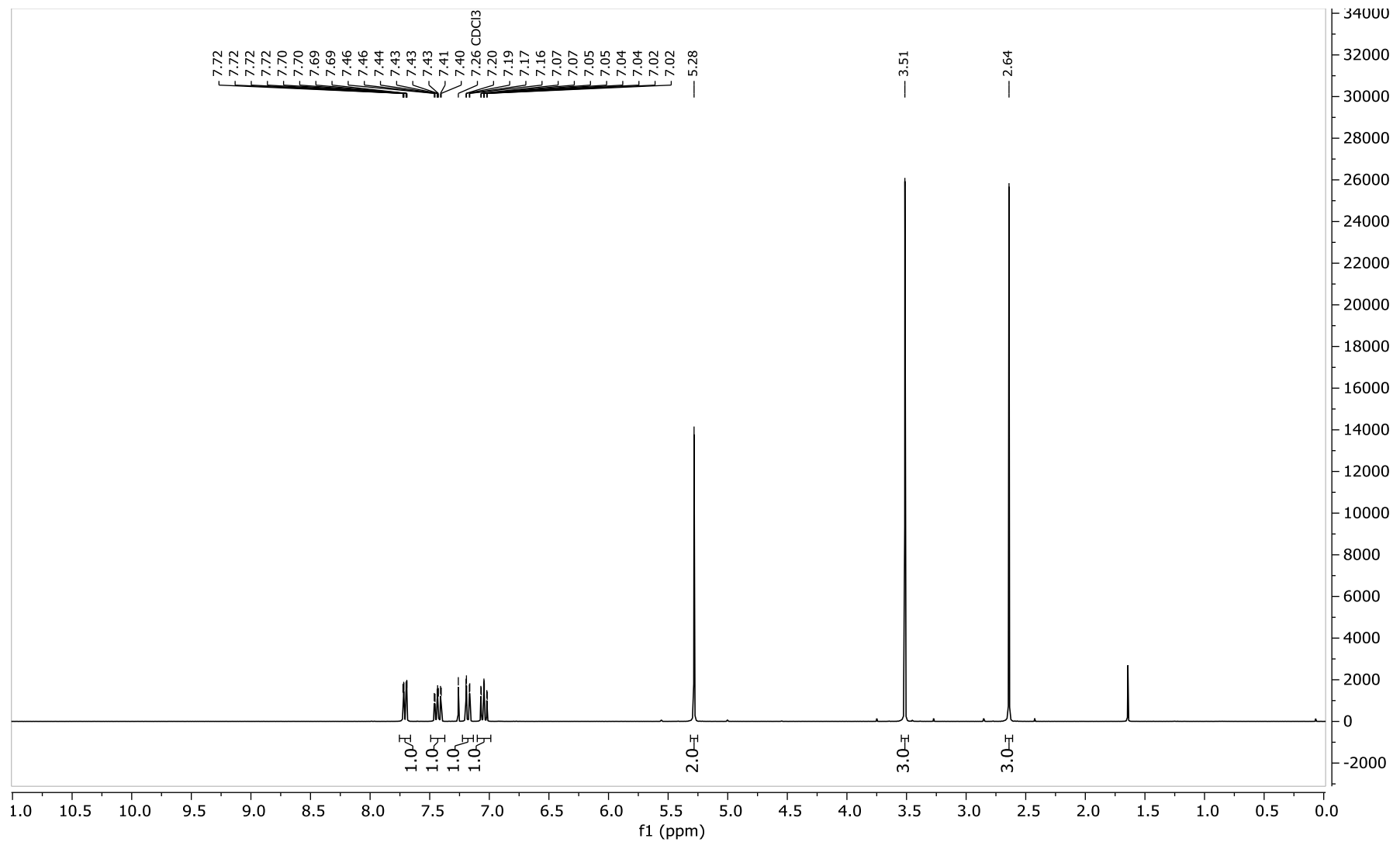

${ }^{13} \mathrm{C} \mathrm{NMR}\left(75 \mathrm{MHz}, \mathrm{CDCl}_{3}\right)$

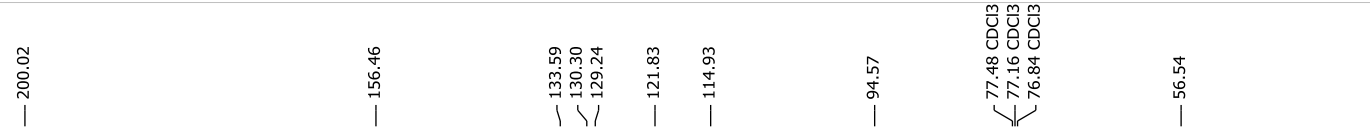

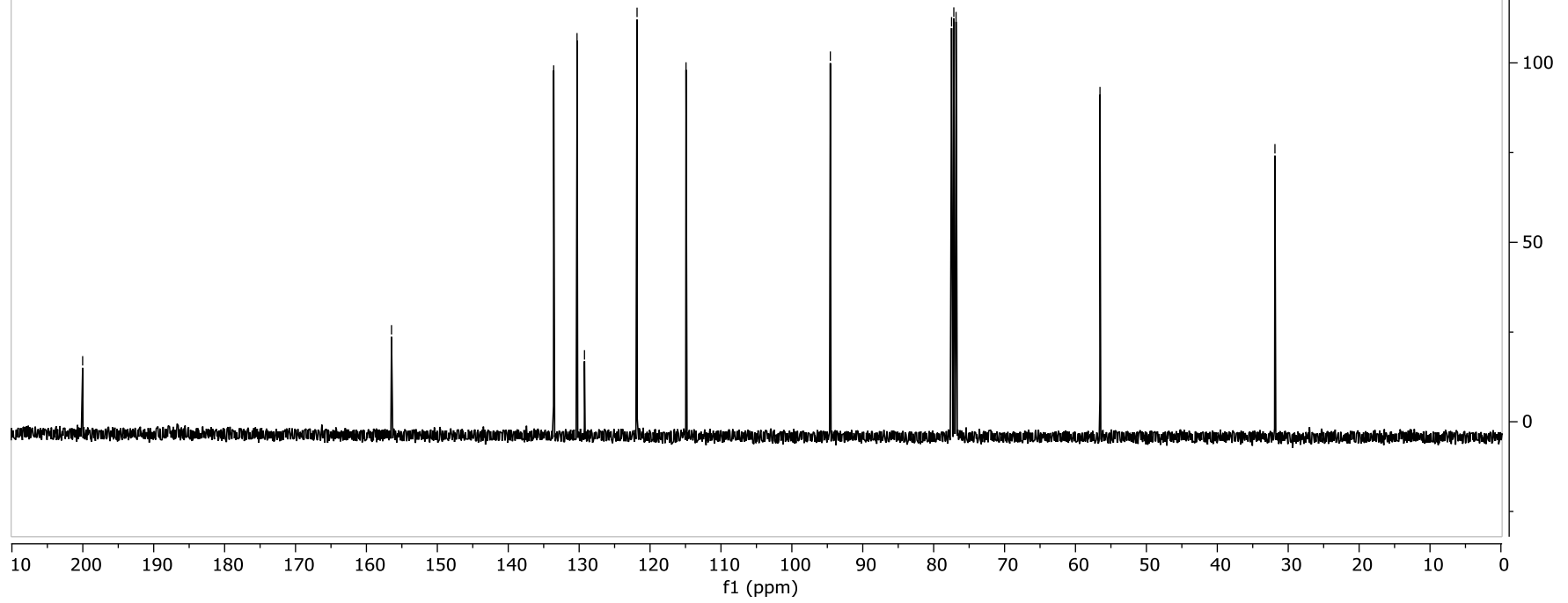




\section{S-23}

${ }^{1} \mathrm{H}$ NMR $\left(300 \mathrm{MHz}, \mathrm{CDCl}_{3}\right)$

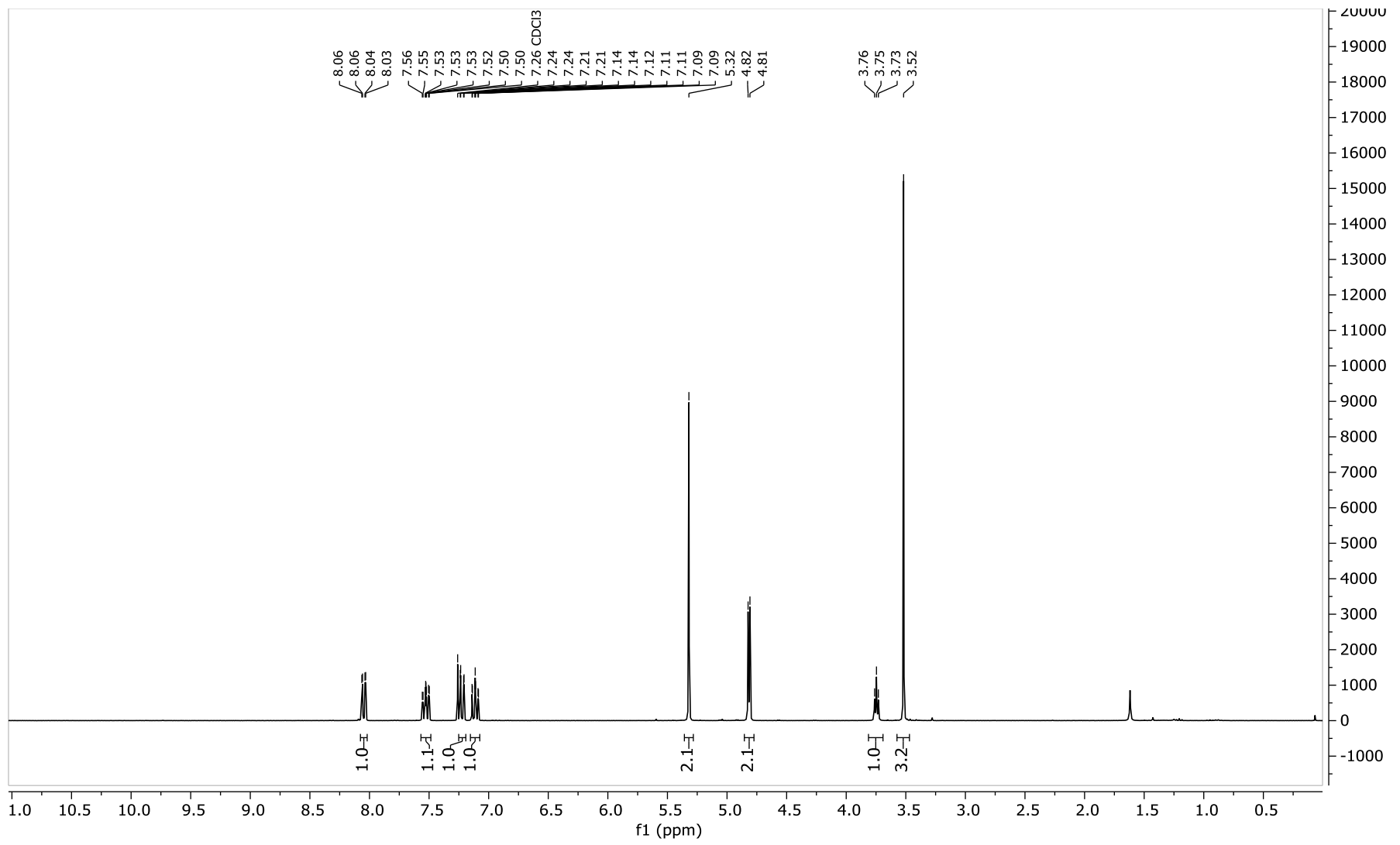

${ }^{13} \mathrm{C}$ NMR (101 MHz, $\mathrm{CDCl}_{3}$ )

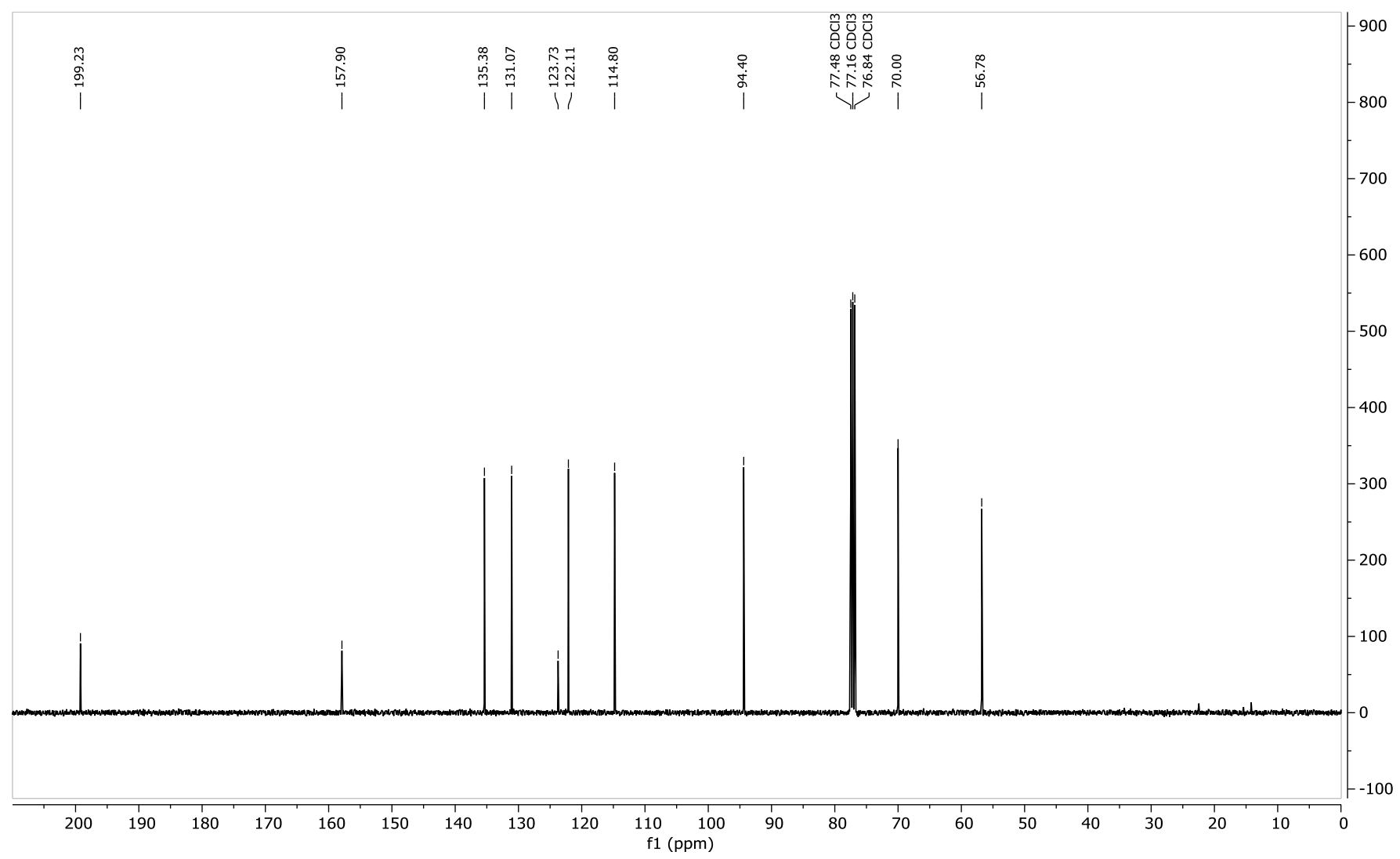




\section{S-24}

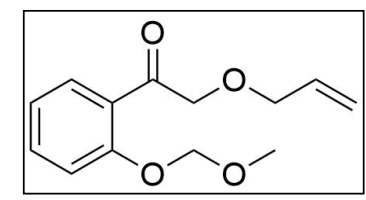

${ }^{1} \mathrm{H}$ NMR (500 MHz, $\mathrm{CDCl}_{3}$ )

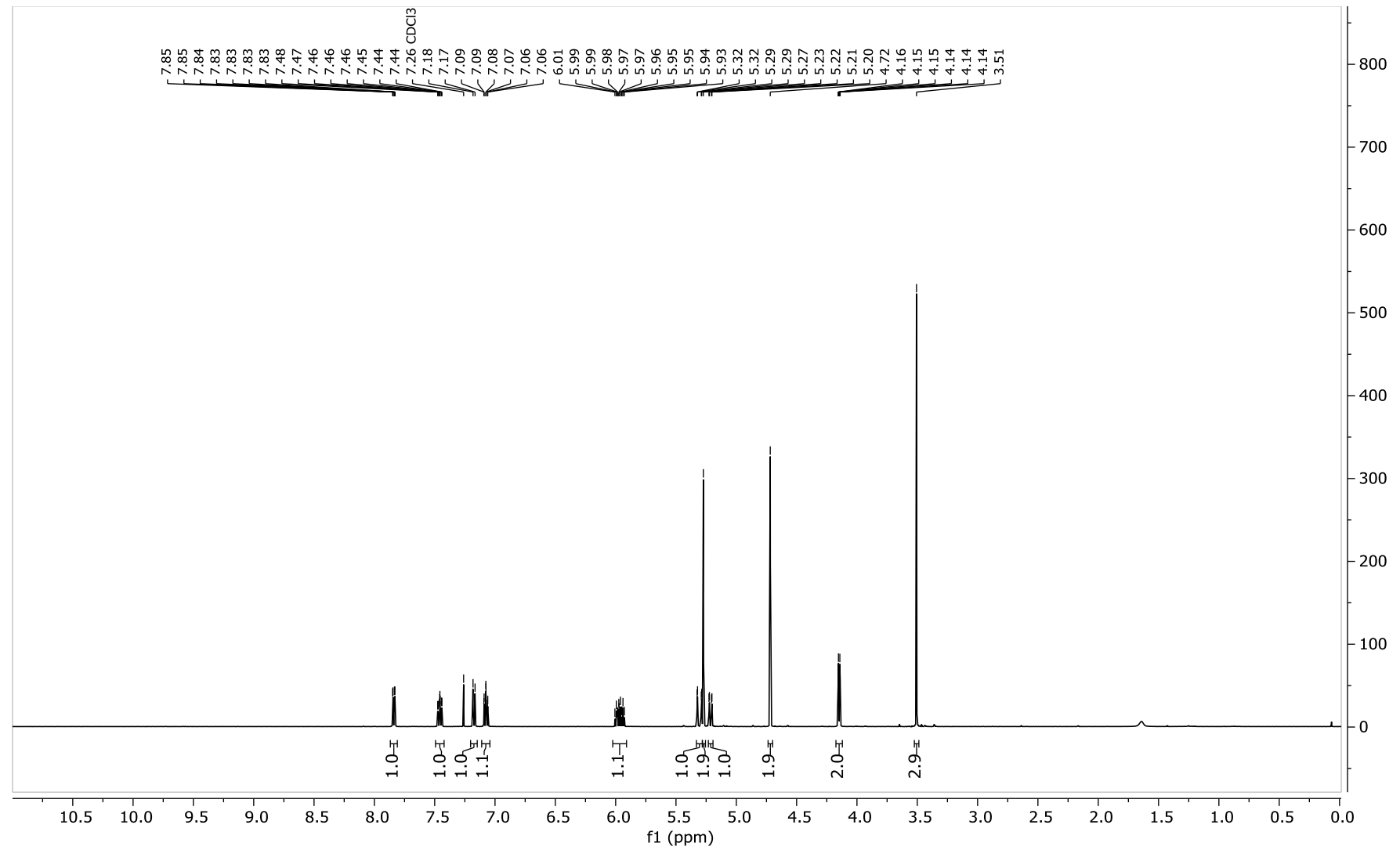

${ }^{13} \mathrm{C}$ NMR (126 MHz, $\mathrm{CDCl}_{3}$ )

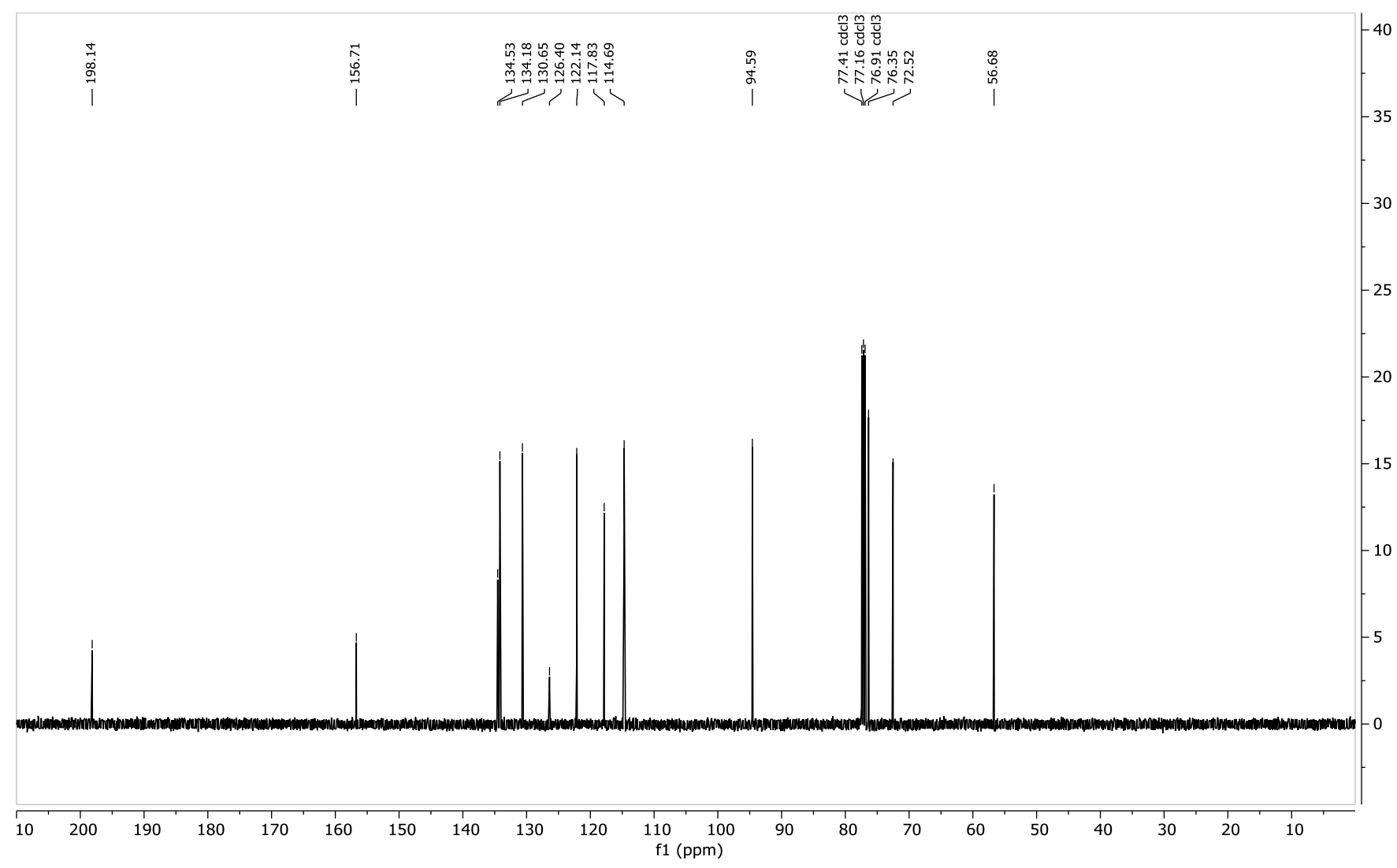




\section{S-25}

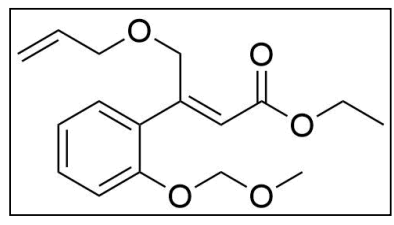

${ }^{1} \mathrm{H}$ NMR $\left(599 \mathrm{MHz}, \mathrm{CDCl}_{3}\right)$

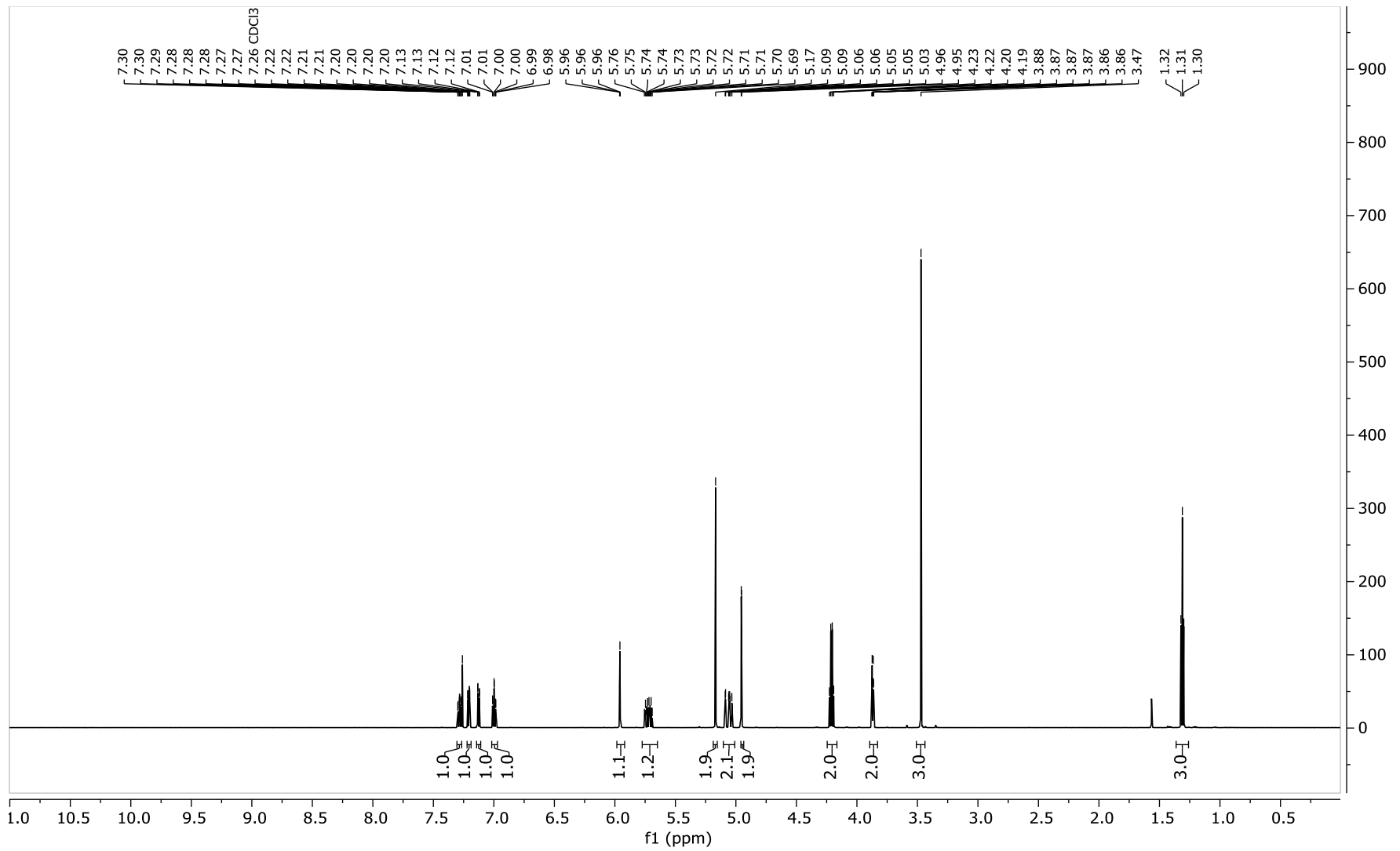

${ }^{13} \mathrm{C}$ NMR $\left(151 \mathrm{MHz}, \mathrm{CDCl}_{3}\right)$

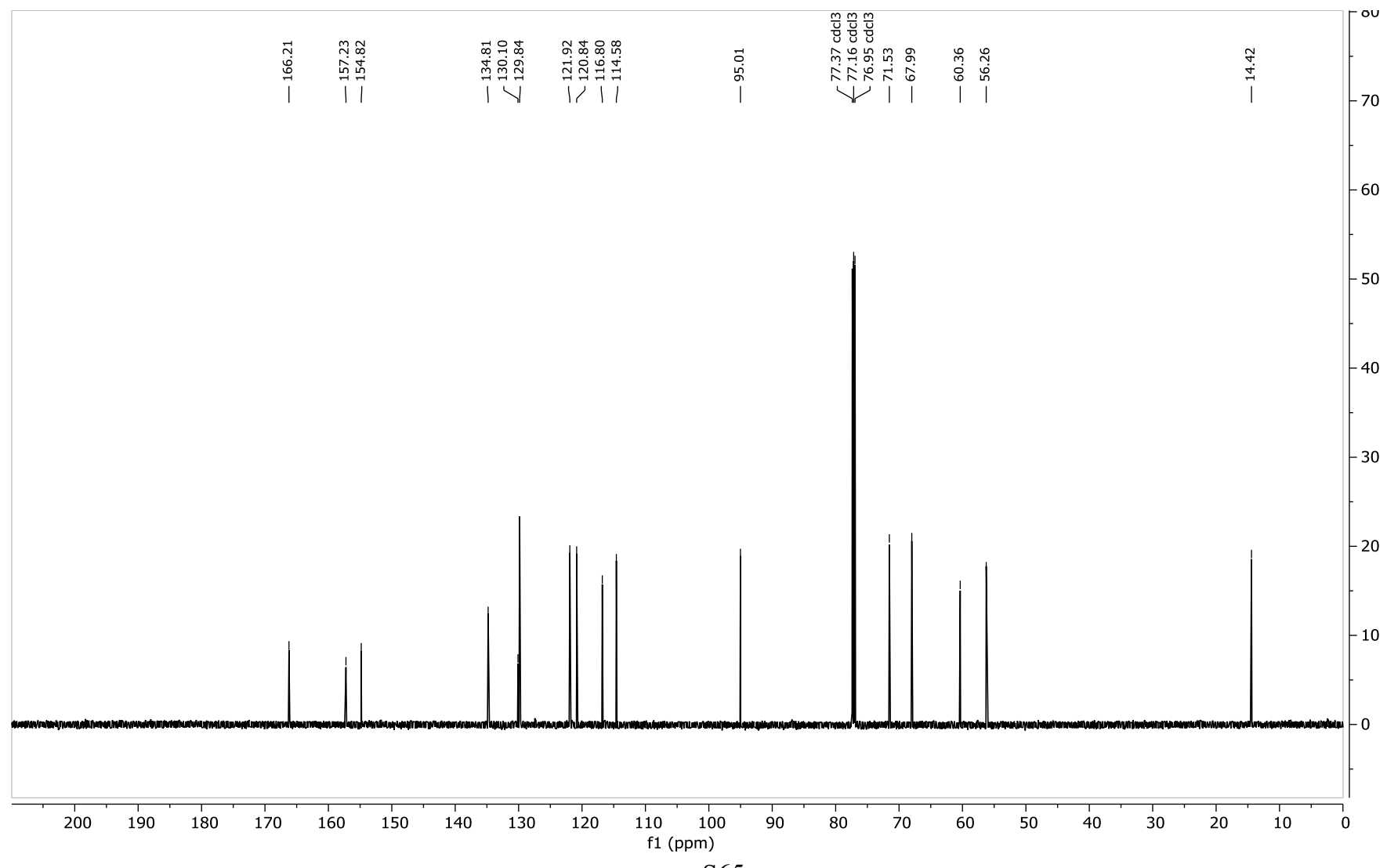




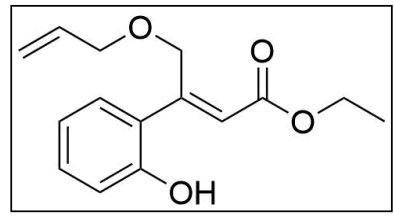

${ }^{1} \mathrm{H}$ NMR (599 MHz, $\mathrm{CDCl}_{3}$ )

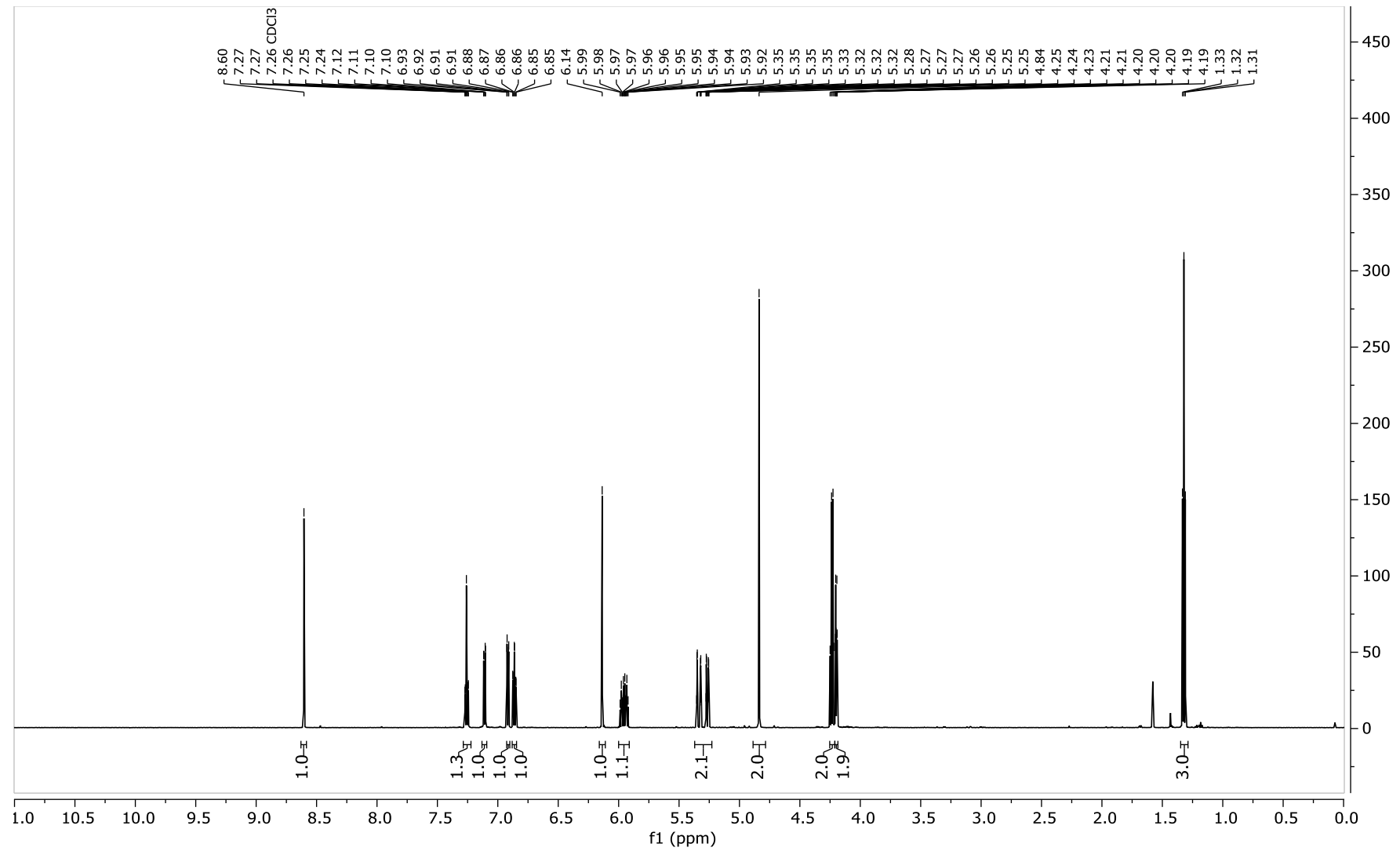

${ }^{13} \mathrm{C}$ NMR (151 MHz, $\mathrm{CDCl}_{3}$ )

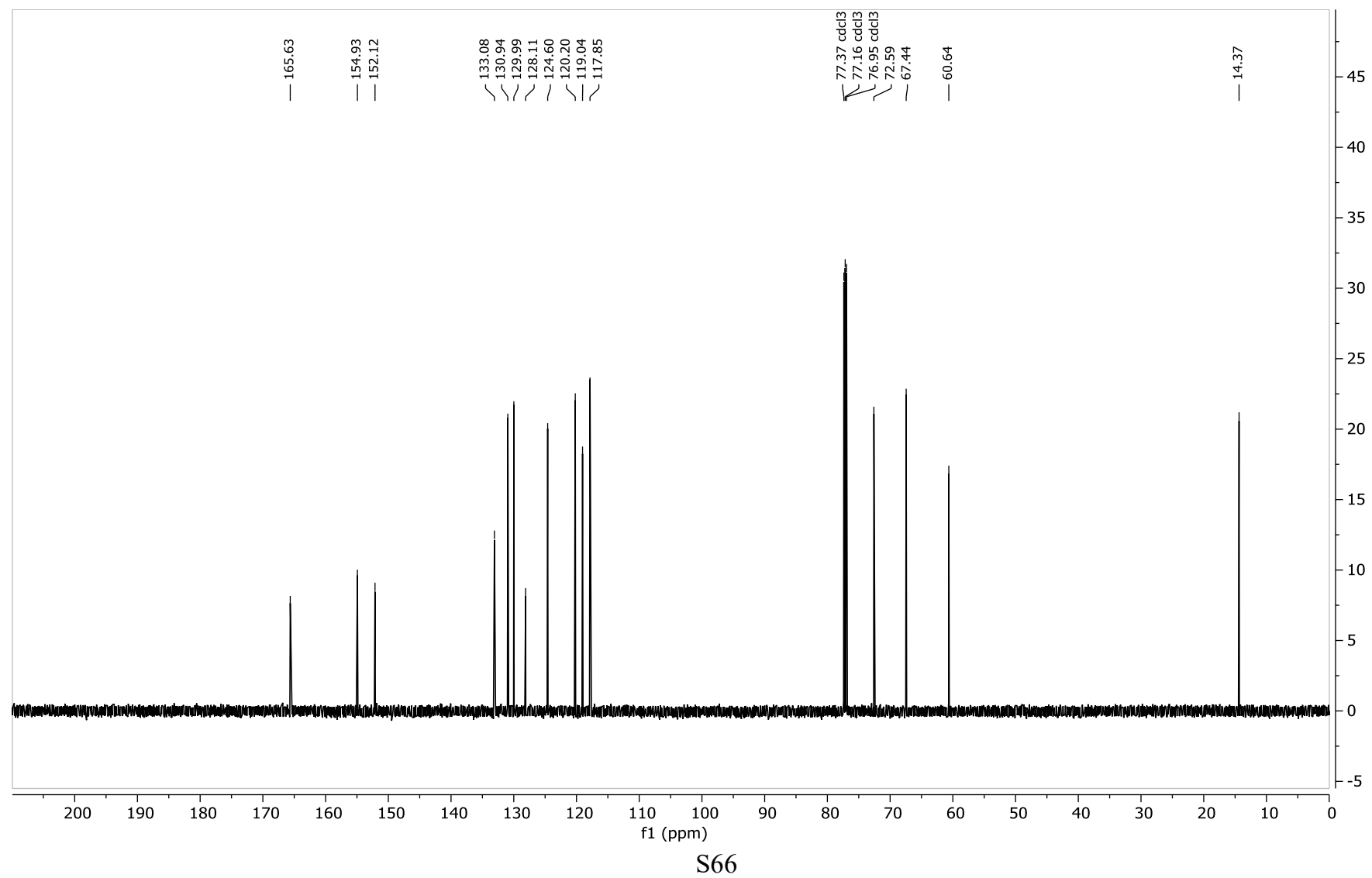




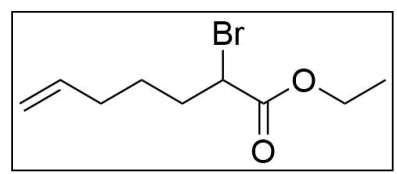

\section{S-27}

${ }^{1} \mathrm{H}$ NMR $\left(300 \mathrm{MHz}, \mathrm{CDCl}_{3}\right)$

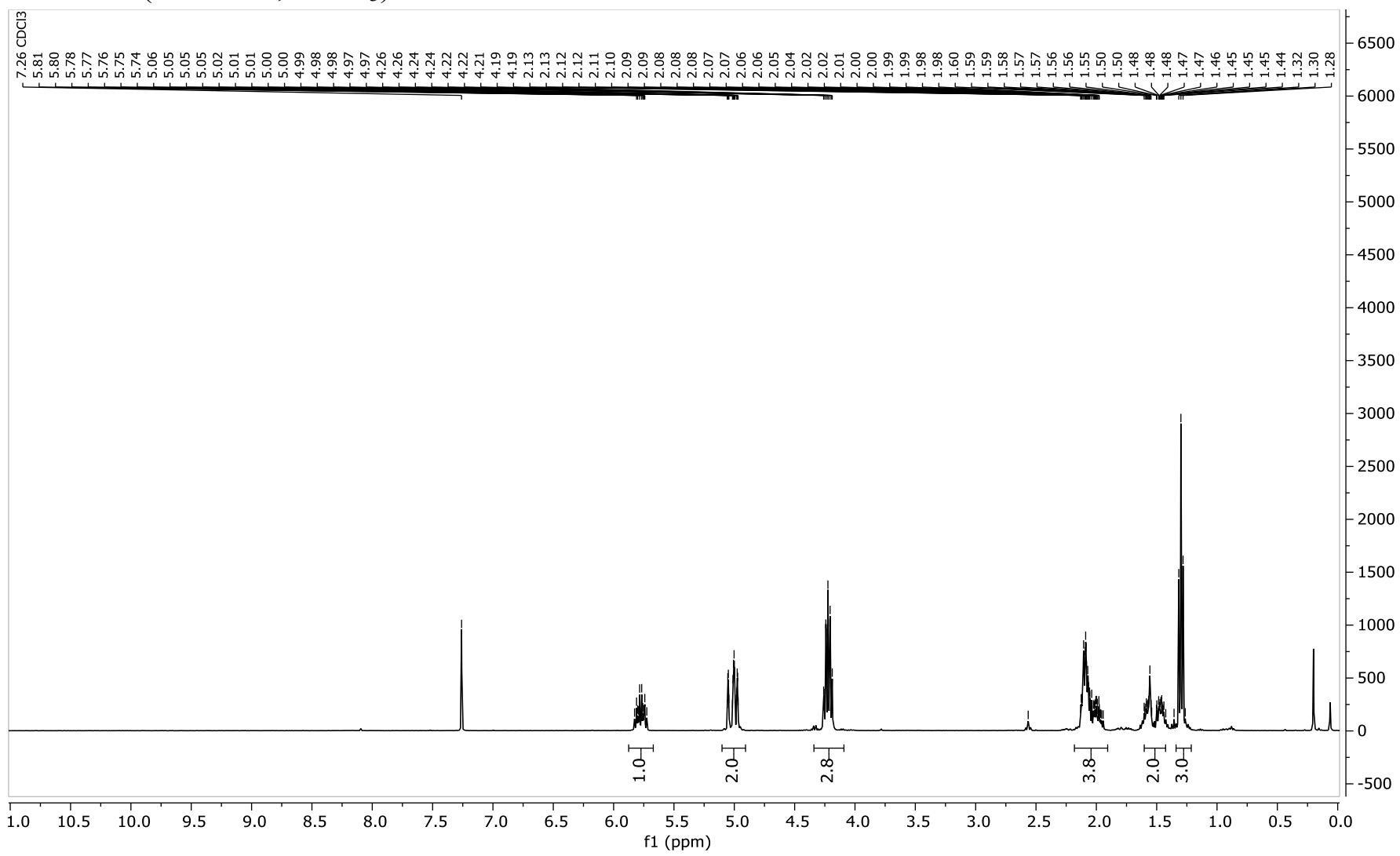

${ }^{13} \mathrm{C} \mathrm{NMR}\left(75 \mathrm{MHz}, \mathrm{CDCl}_{3}\right)$

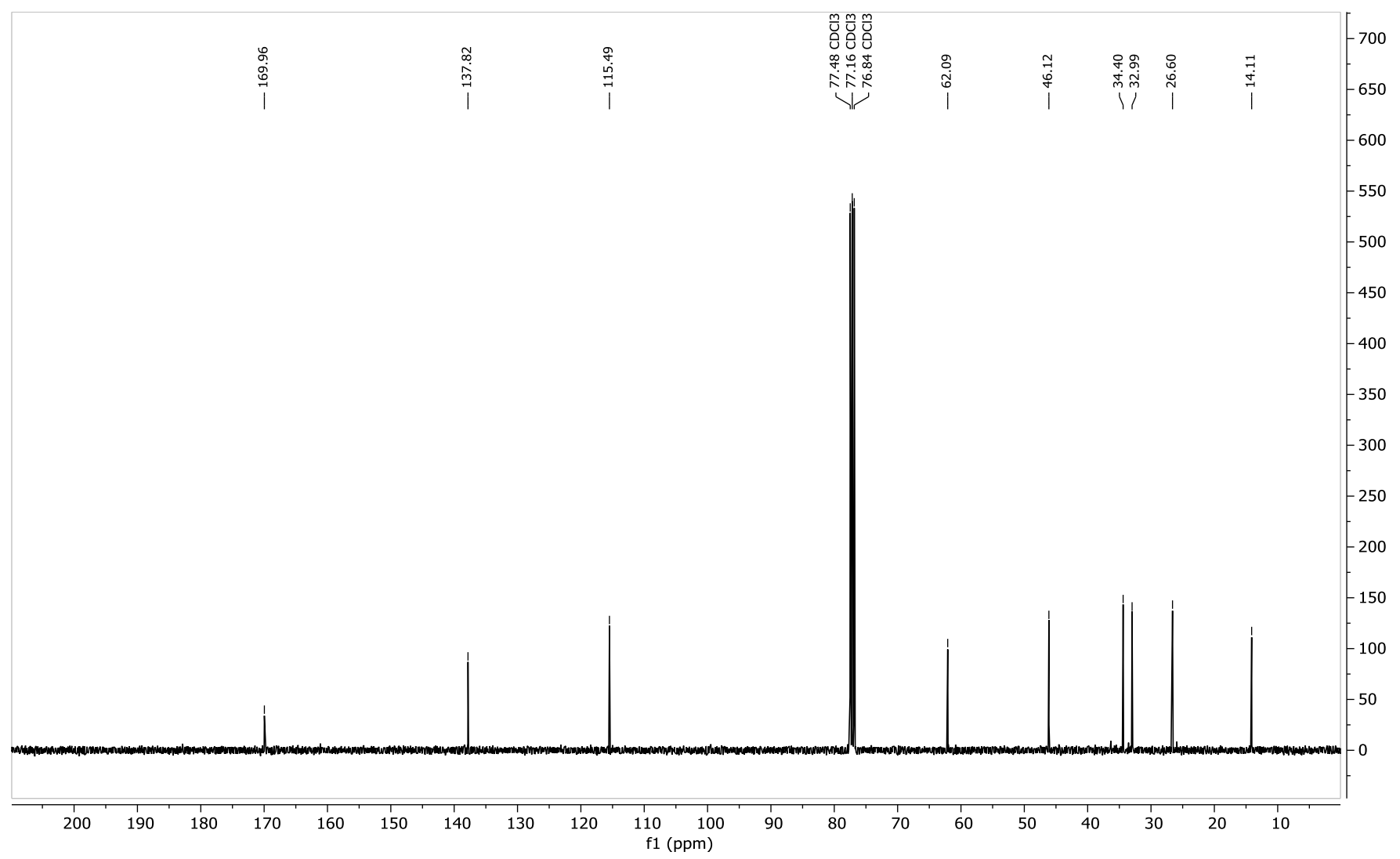


${ }^{1} \mathrm{H}$ NMR (599 MHz, $\mathrm{CDCl}_{3}$ )
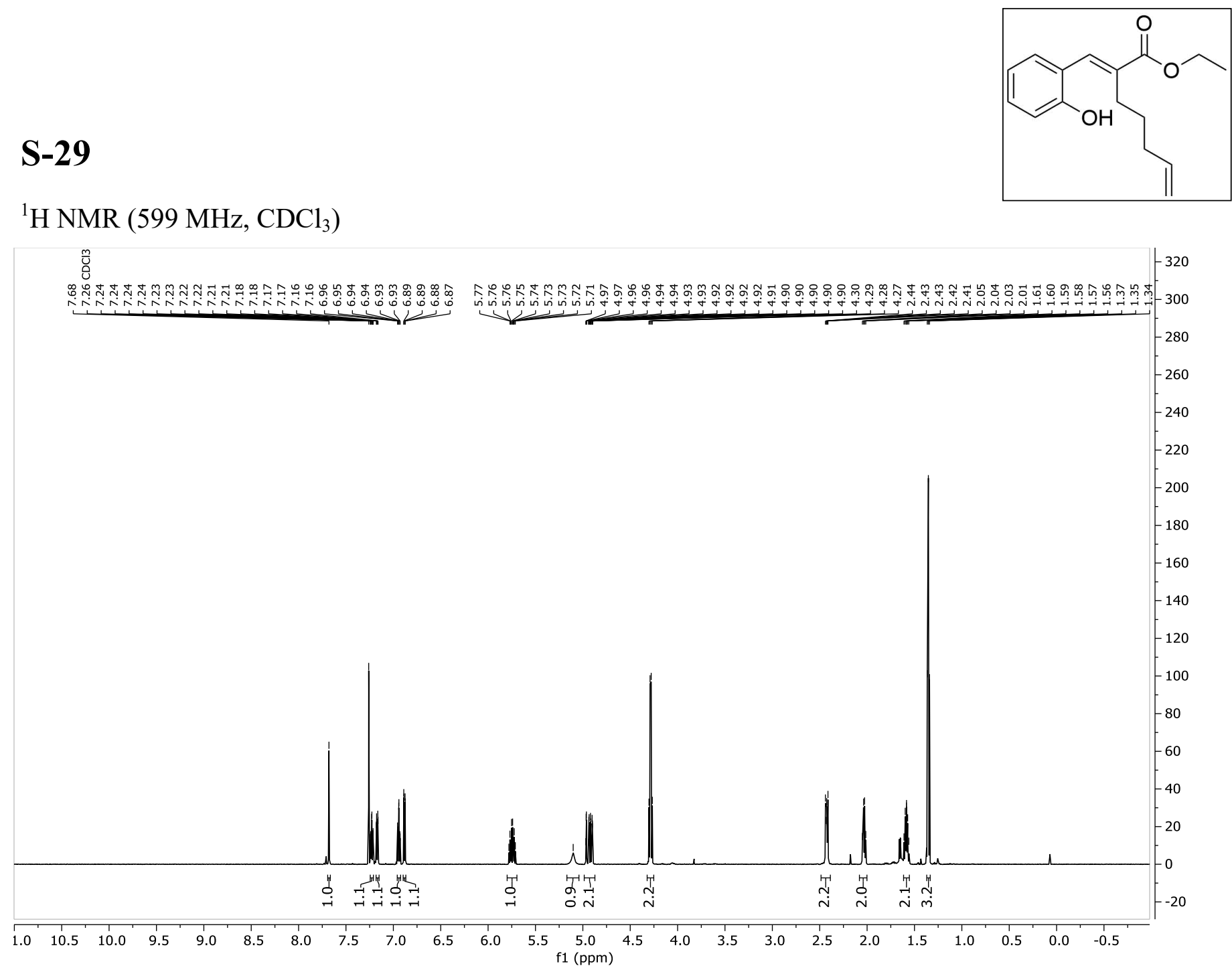

${ }^{13} \mathrm{C}$ NMR (151 MHz, $\mathrm{CDCl}_{3}$ )

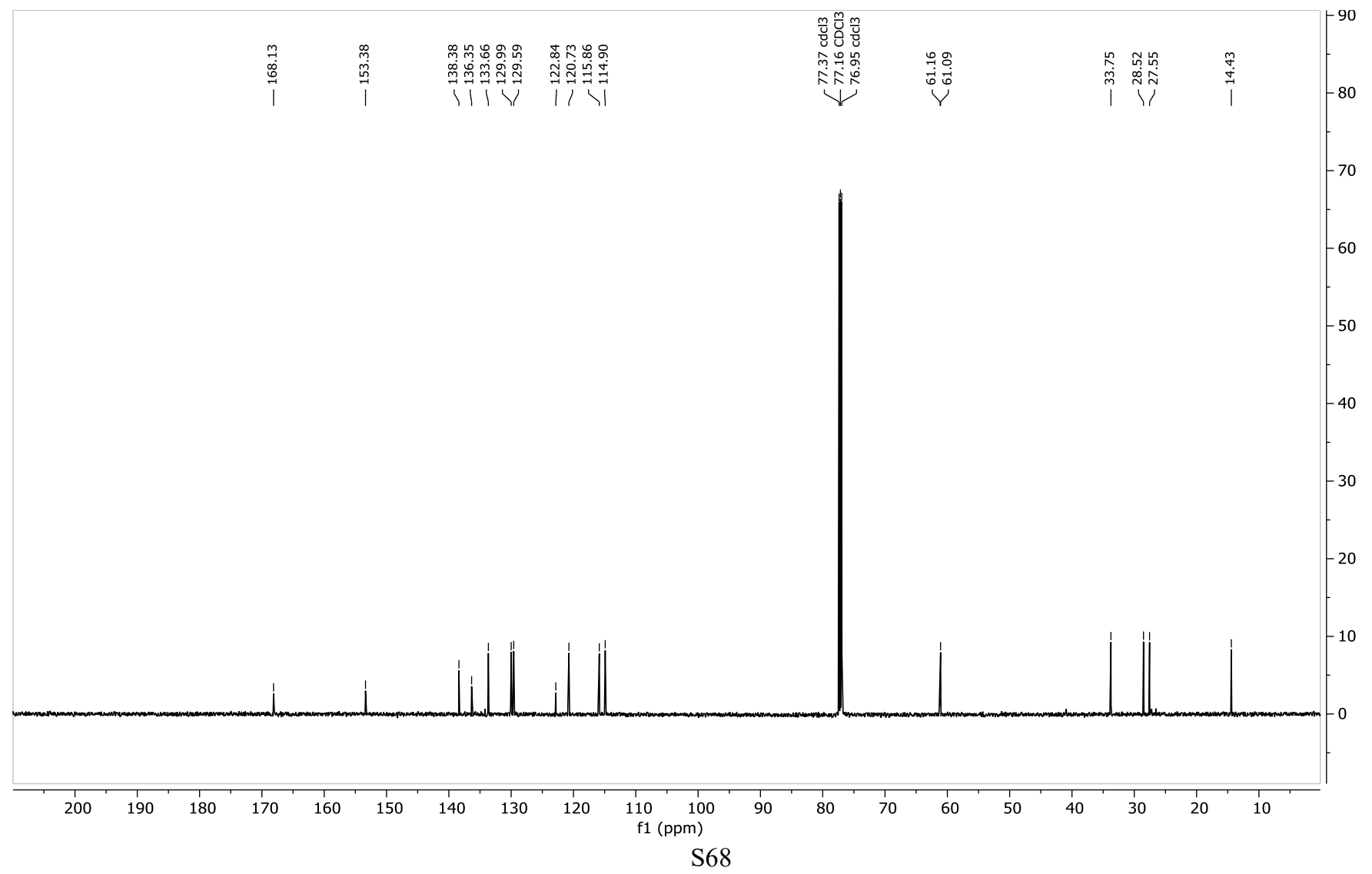


${ }^{1} \mathrm{H}$ NMR (599 MHz, $\mathrm{CDCl}_{3}$ )

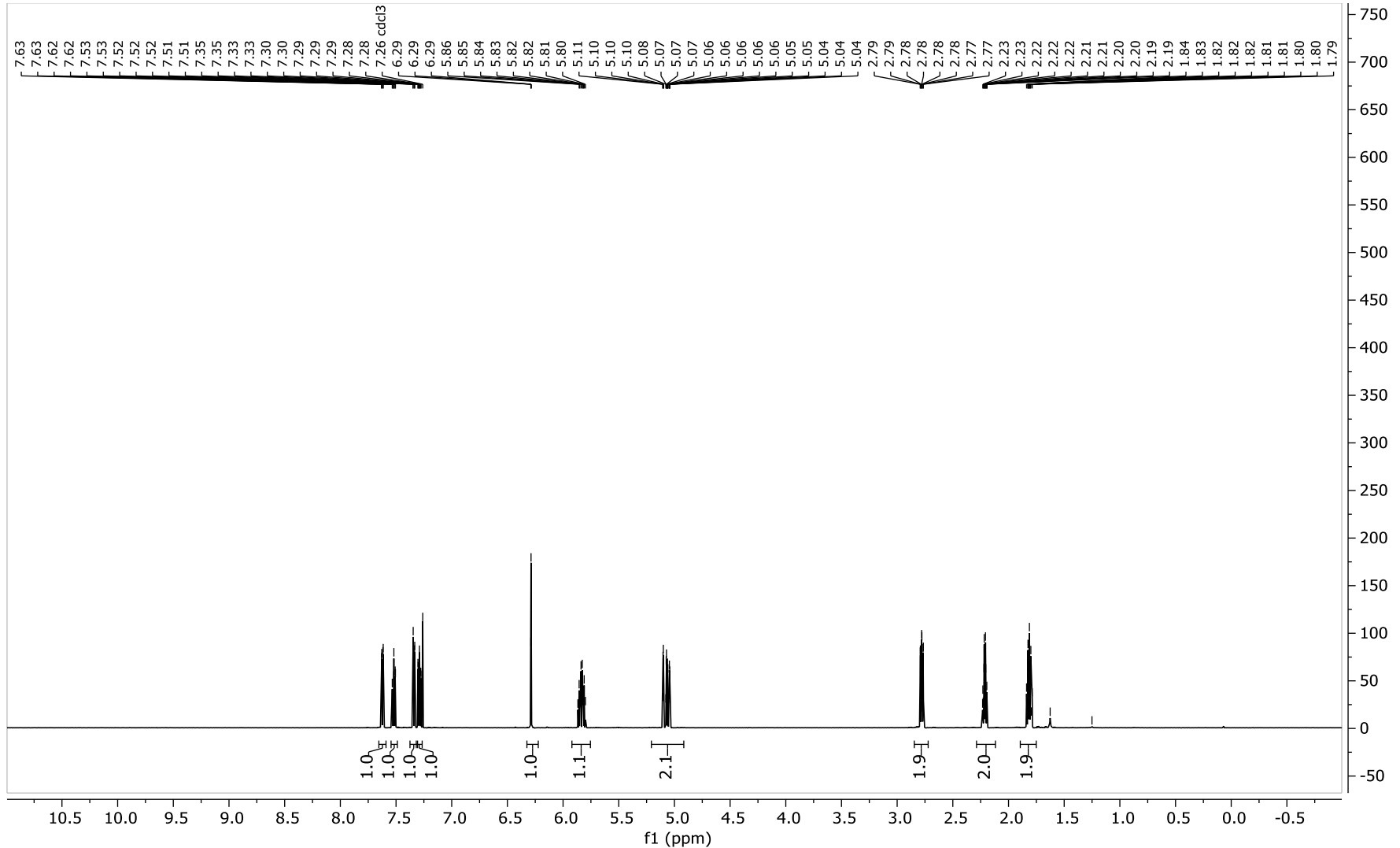

${ }^{13} \mathrm{C}$ NMR (151 MHz, $\mathrm{CDCl}_{3}$ )

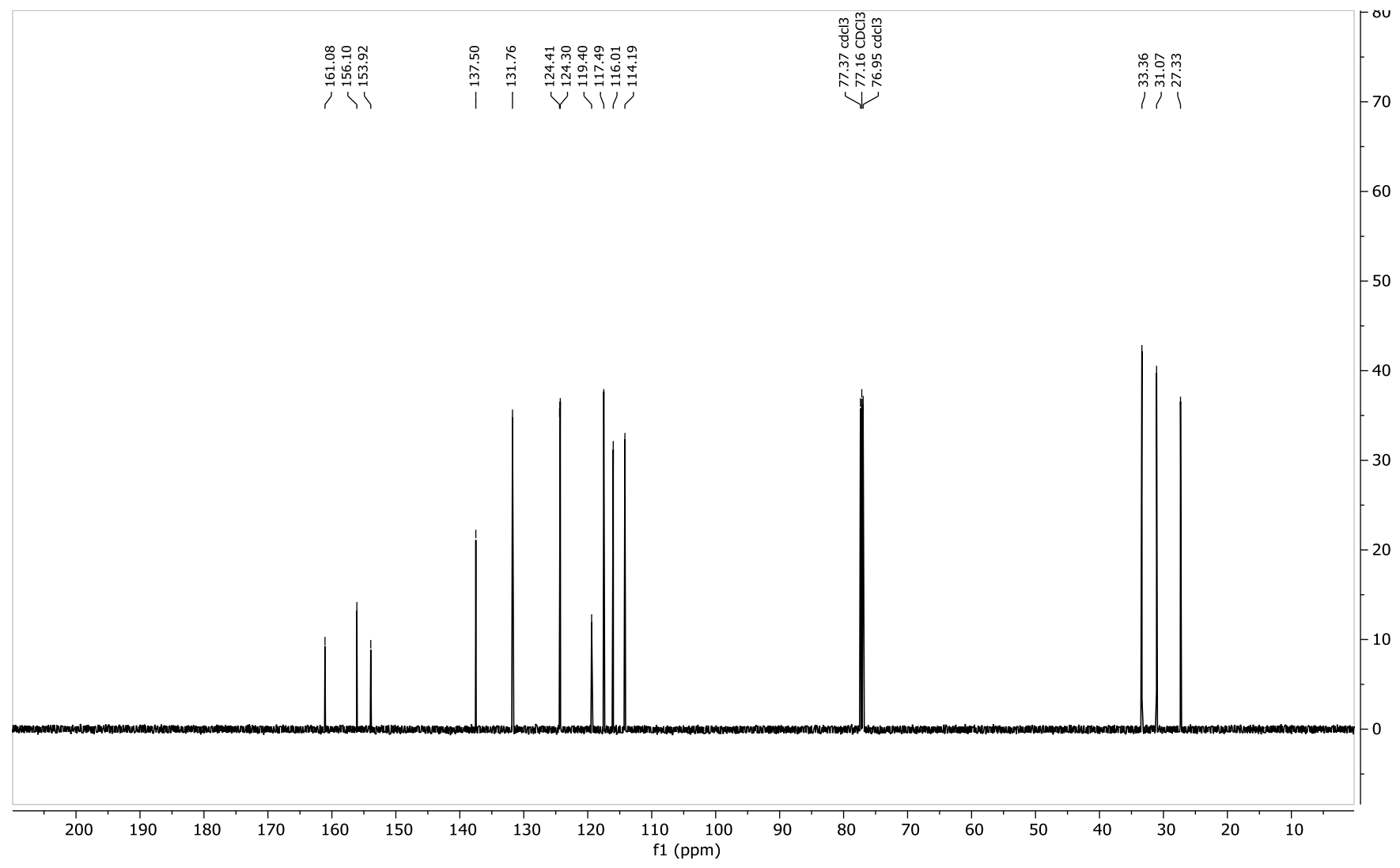


S-30

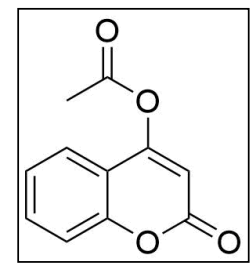

${ }^{1} \mathrm{H}$ NMR $\left(400 \mathrm{MHz}, \mathrm{CDCl}_{3}\right)$

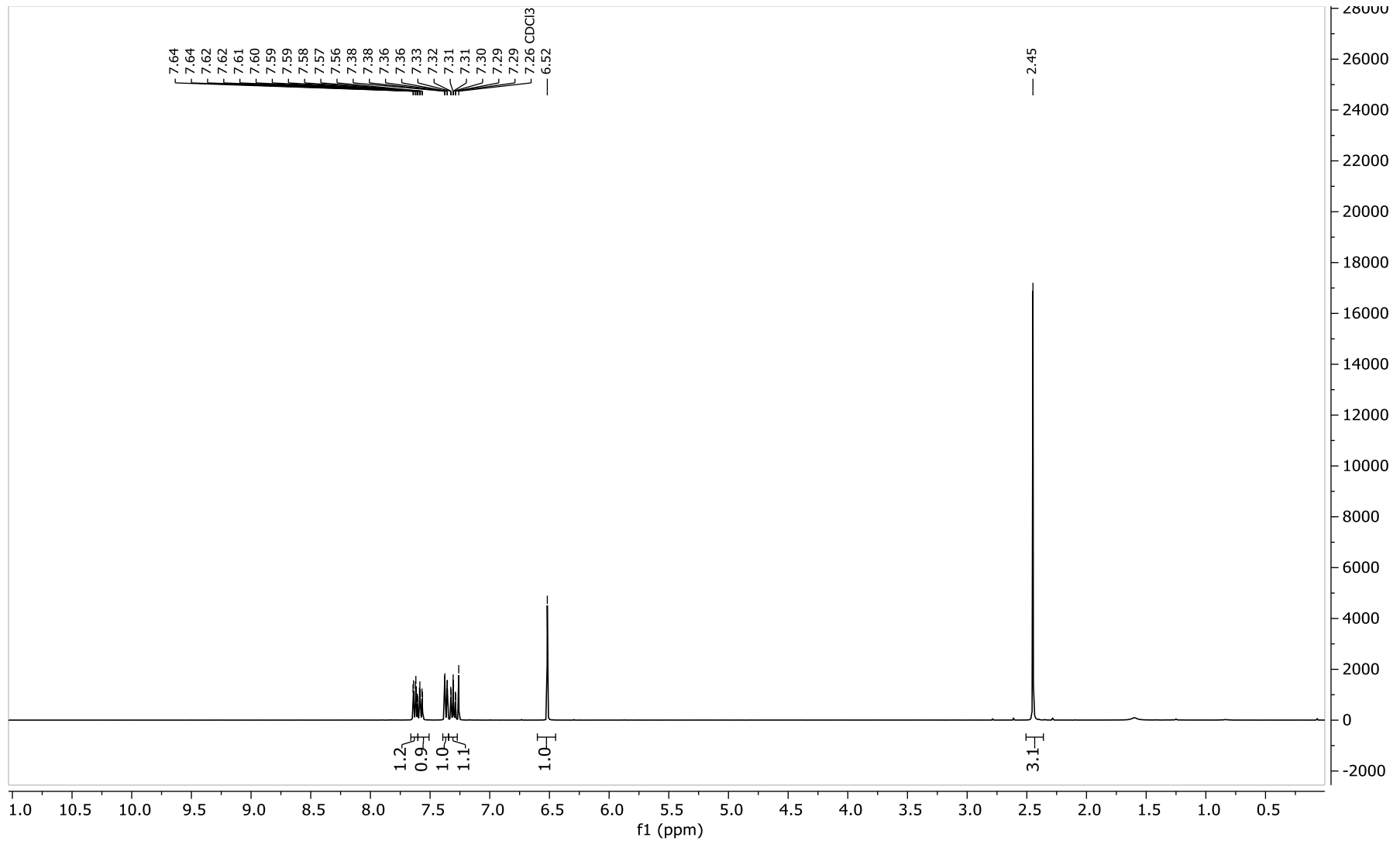

${ }^{13} \mathrm{C}$ NMR $\left(101 \mathrm{MHz}, \mathrm{CDCl}_{3}\right)$

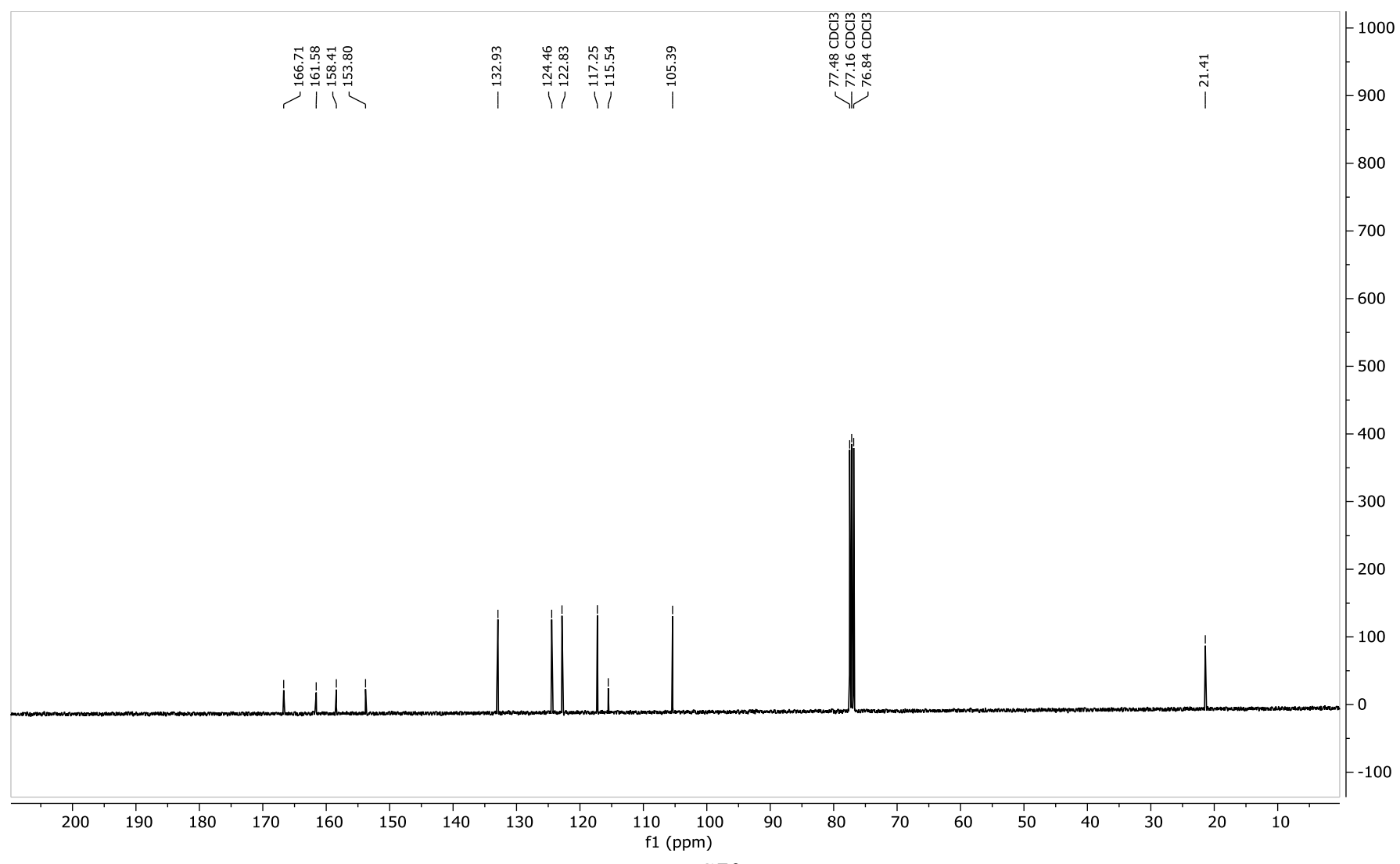


${ }^{1} \mathrm{H}$ NMR (599 MHz, $\mathrm{CDCl}_{3}$ )

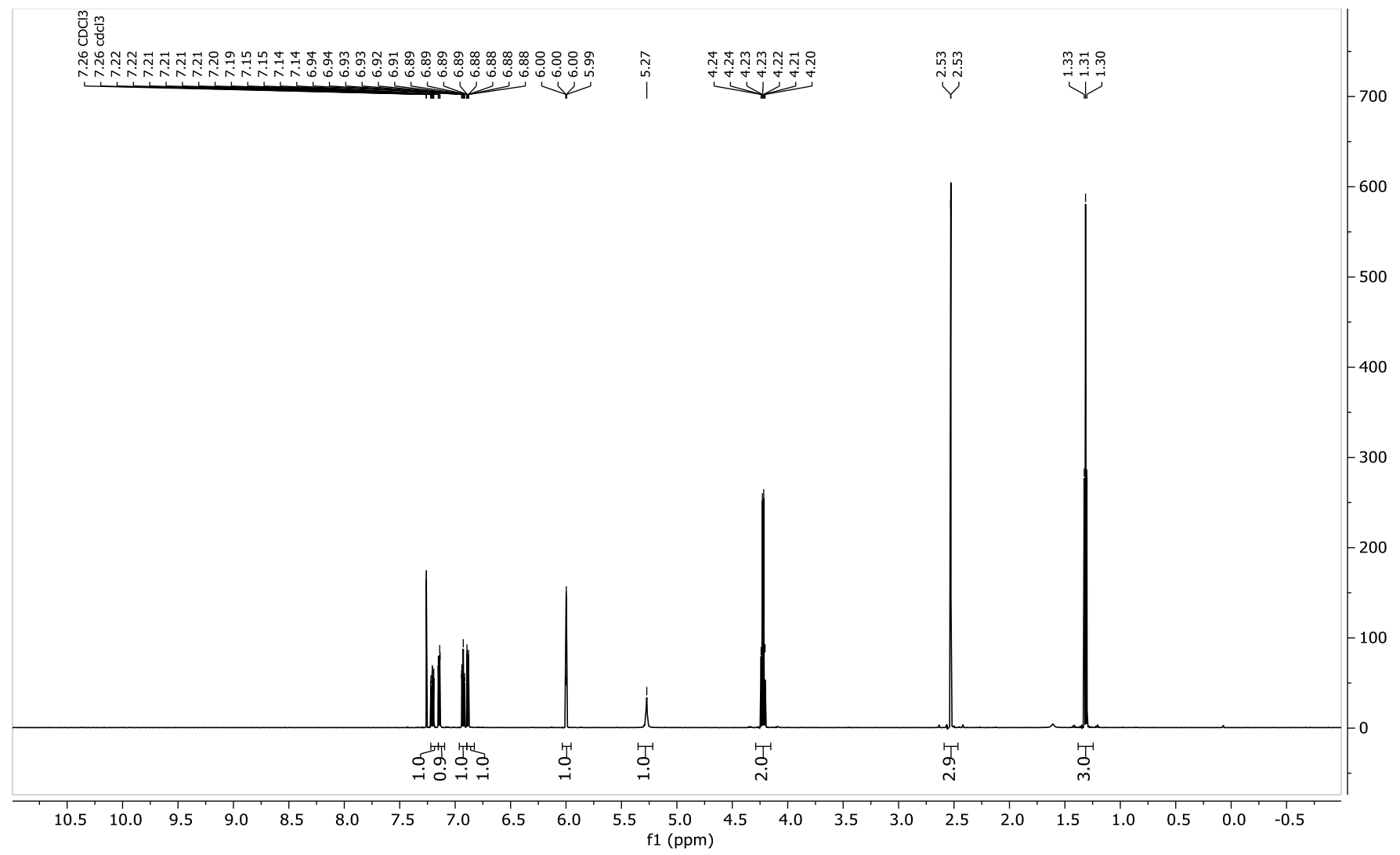

${ }^{13} \mathrm{C}$ NMR (151 MHz, $\mathrm{CDCl}_{3}$ )

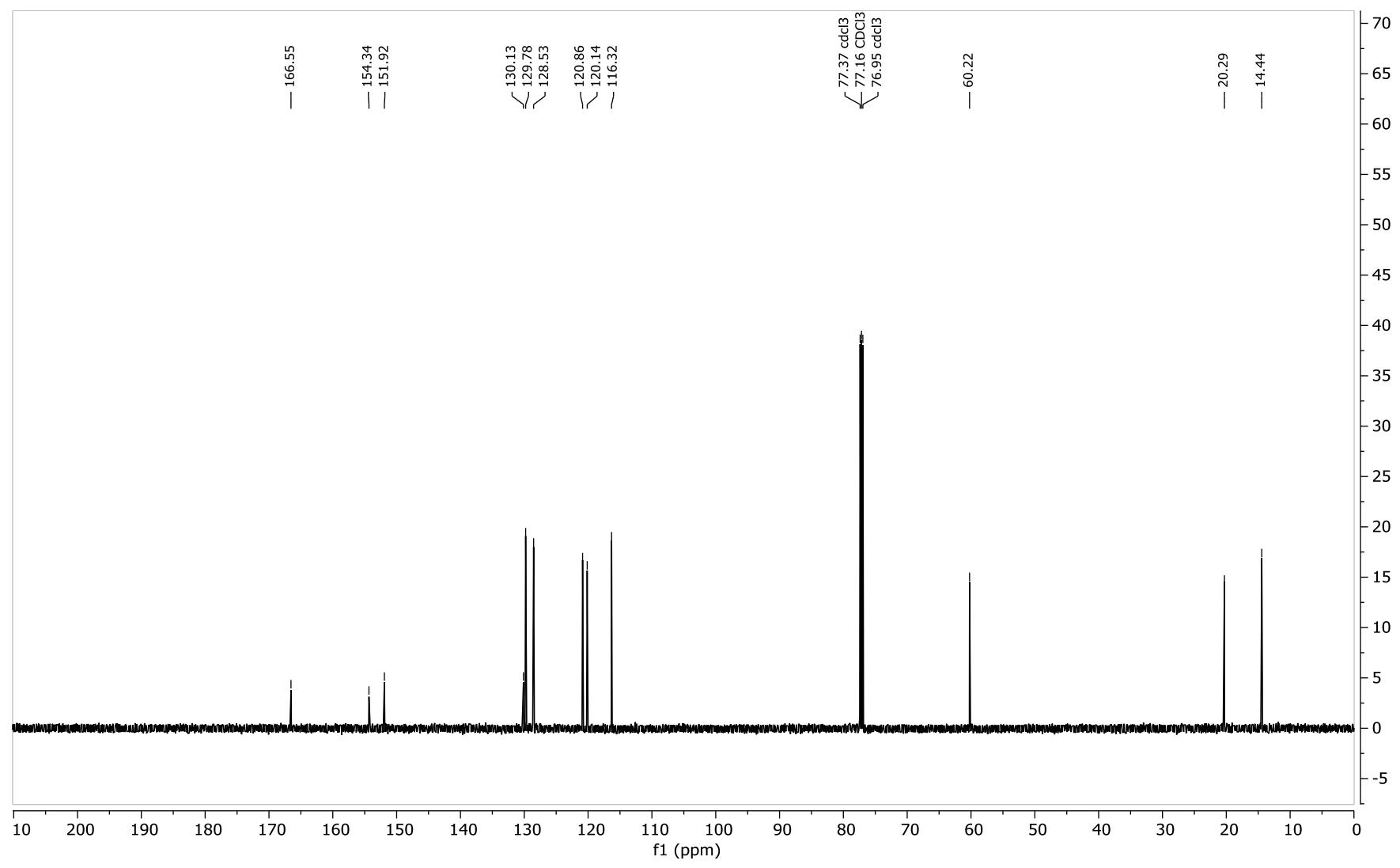


${ }^{1} \mathrm{H}$ NMR (599 MHz, $\mathrm{CDCl}_{3}$ )

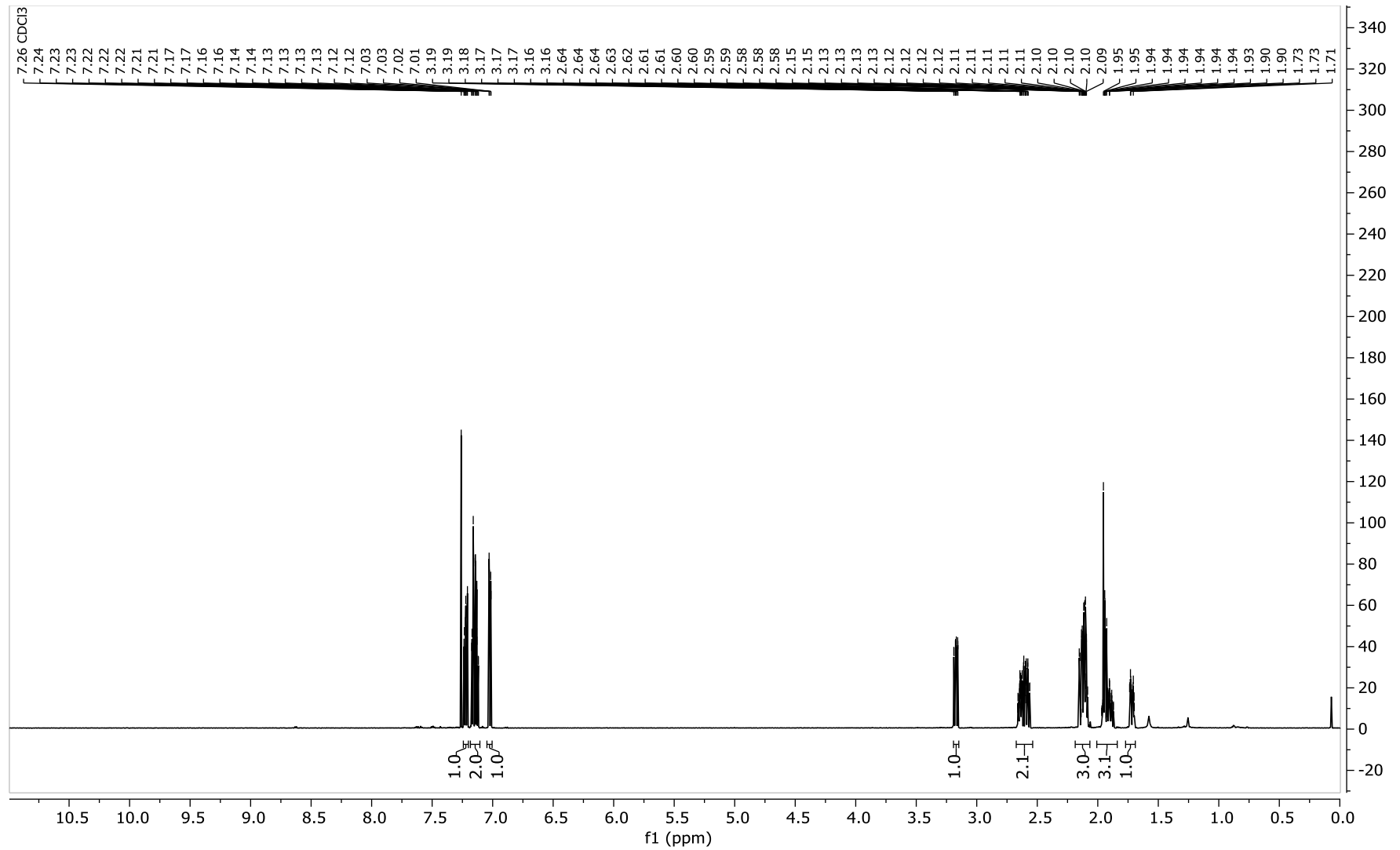

${ }^{13} \mathrm{C}$ NMR (151 MHz, $\left.\mathrm{CDCl}_{3}\right)$

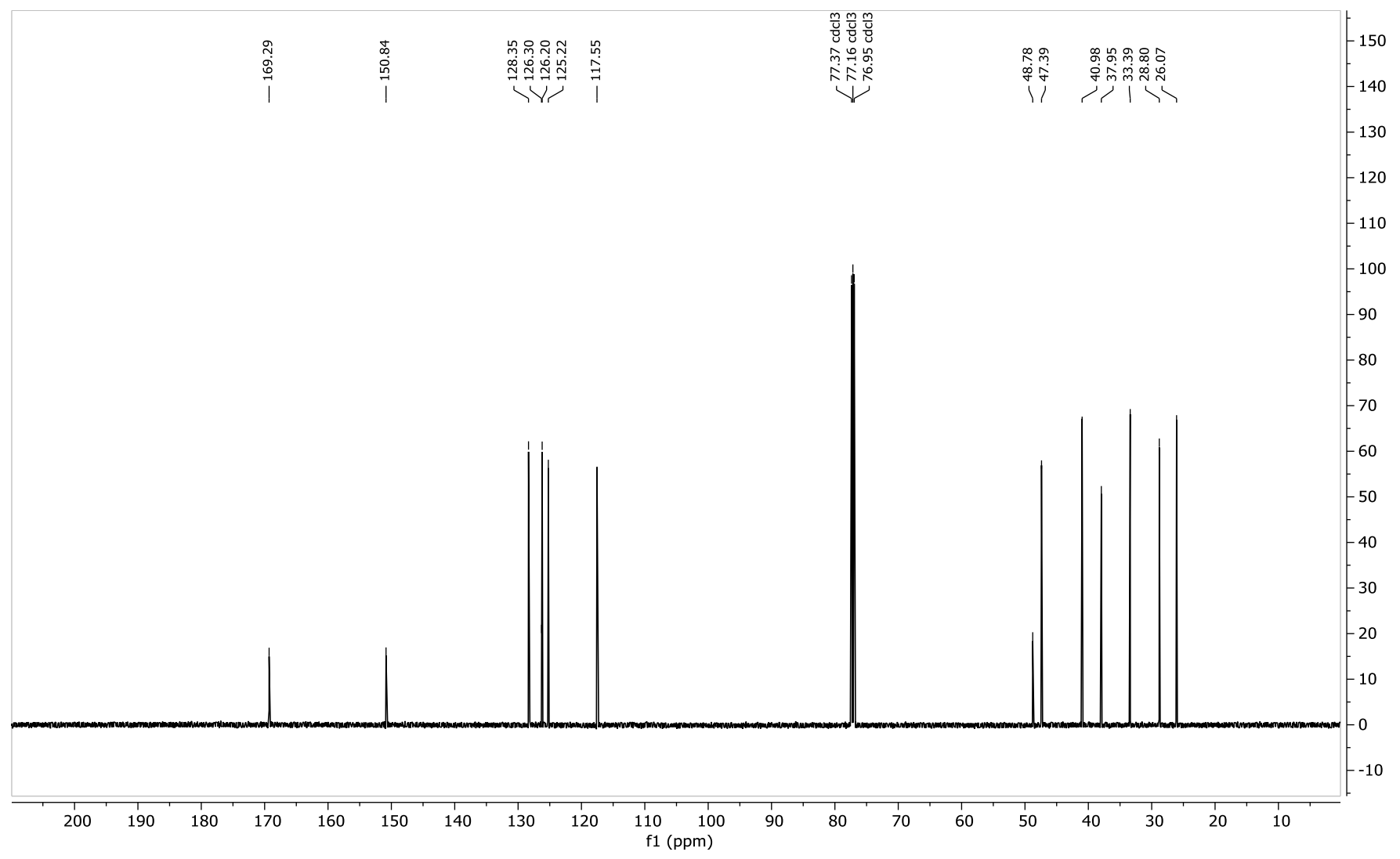


${ }^{1} \mathrm{H}$ NMR (599 MHz, $\mathrm{CDCl}_{3}$ )

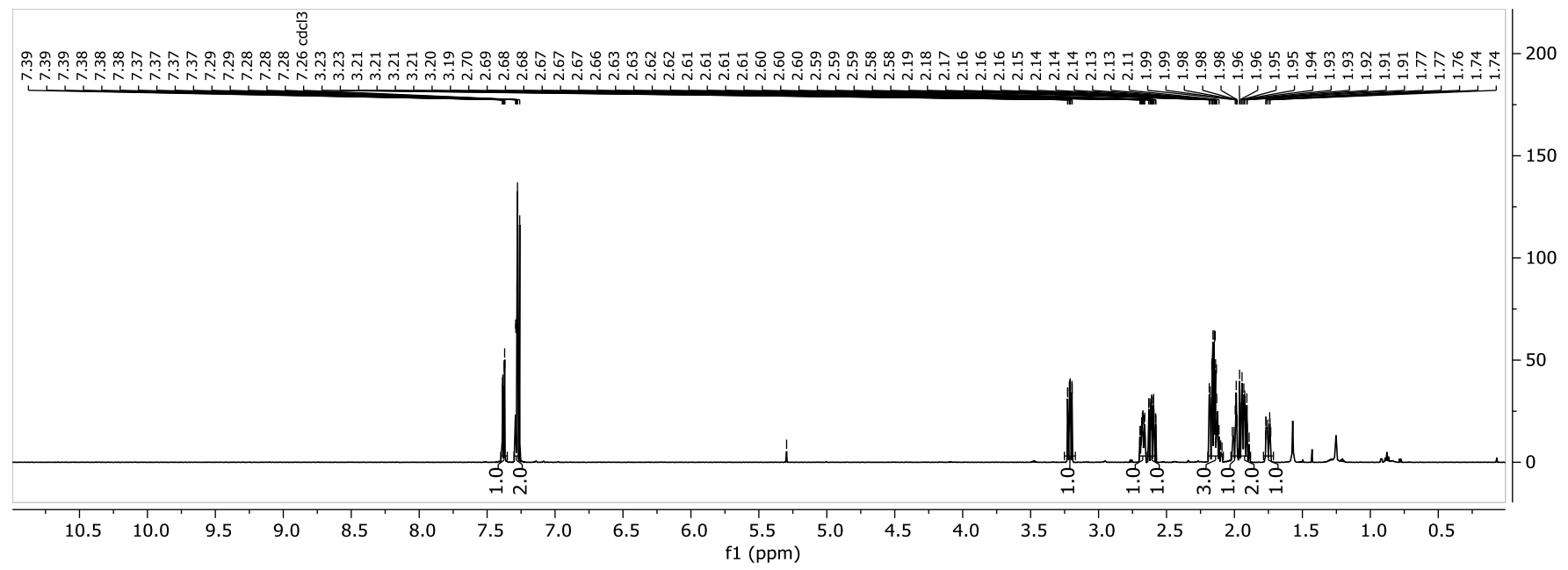

${ }^{13} \mathrm{C} \mathrm{NMR}\left(151 \mathrm{MHz}, \mathrm{CDCl}_{3}\right)$

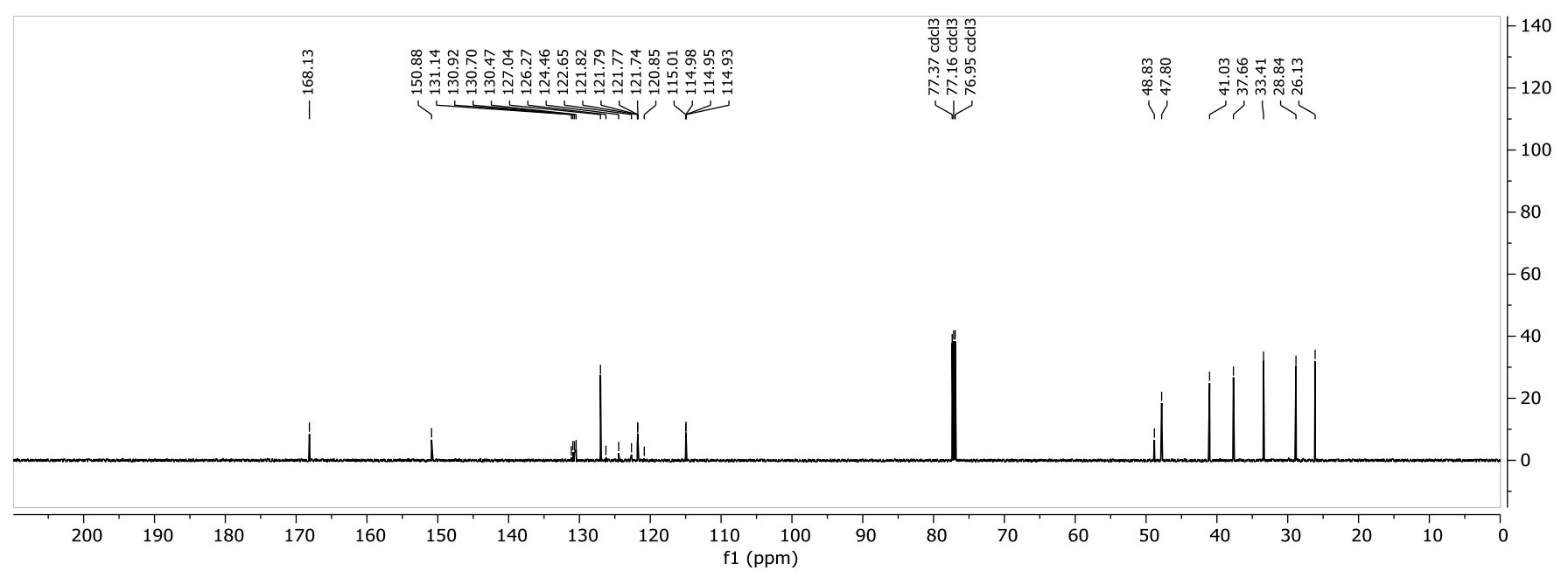

${ }^{19} \mathrm{~F}$ NMR (564 MHz, $\left.\mathrm{CDCl}_{3}\right)$

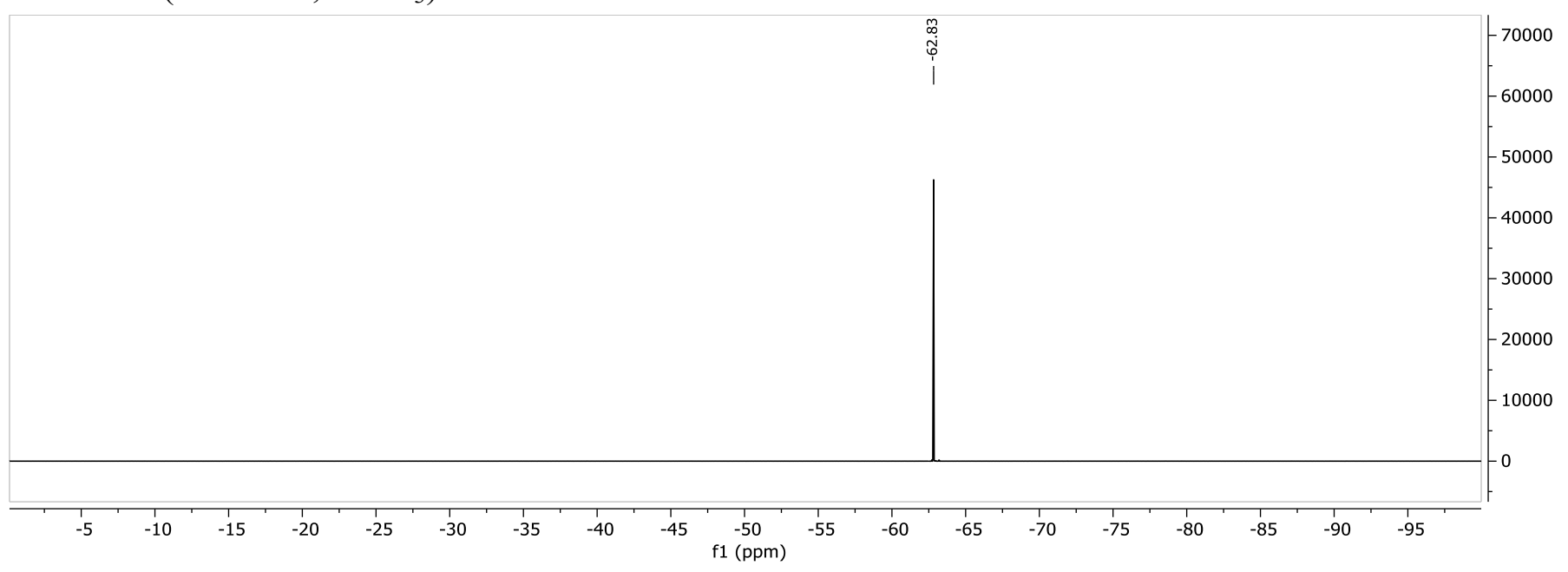




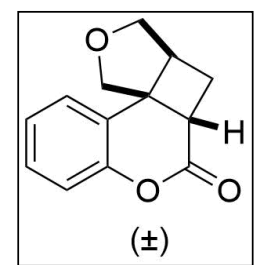

${ }^{1} \mathrm{H}$ NMR (599 MHz, $\mathrm{CDCl}_{3}$ )

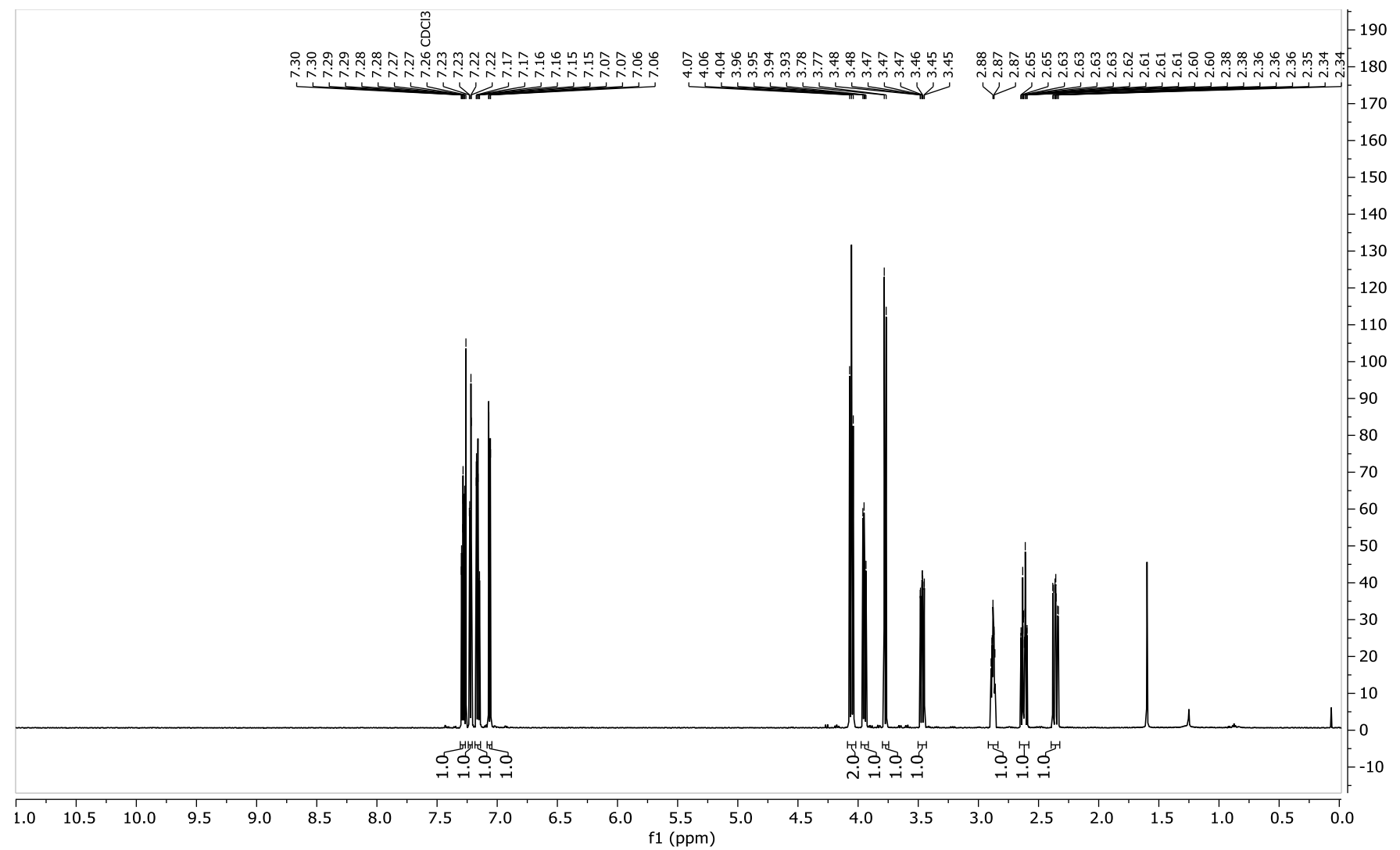

${ }^{13} \mathrm{C}$ NMR (151 MHz, $\left.\mathrm{CDCl}_{3}\right)$

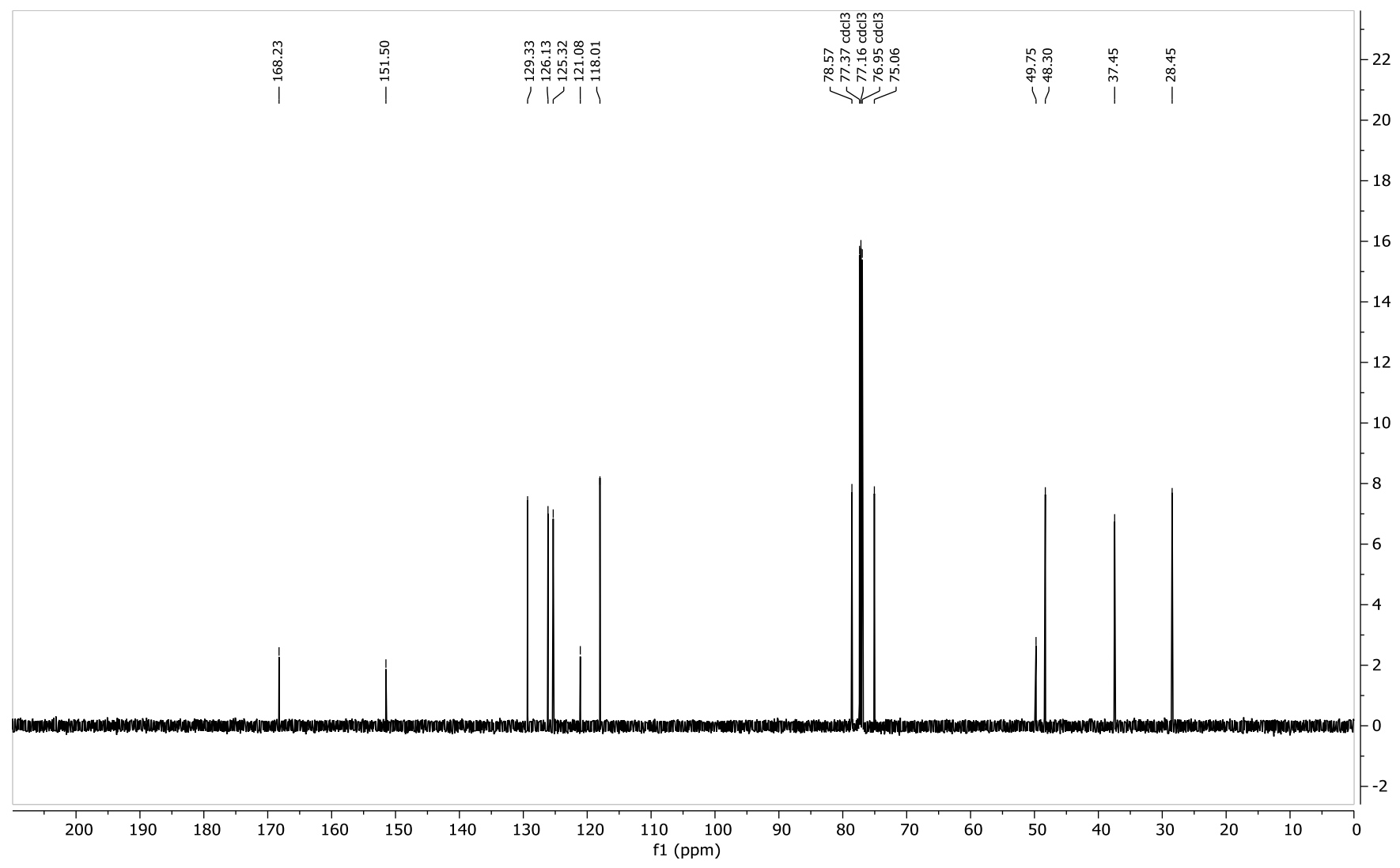


${ }^{1} \mathrm{H}$ NMR (599 MHz, $\mathrm{CDCl}_{3}$ )

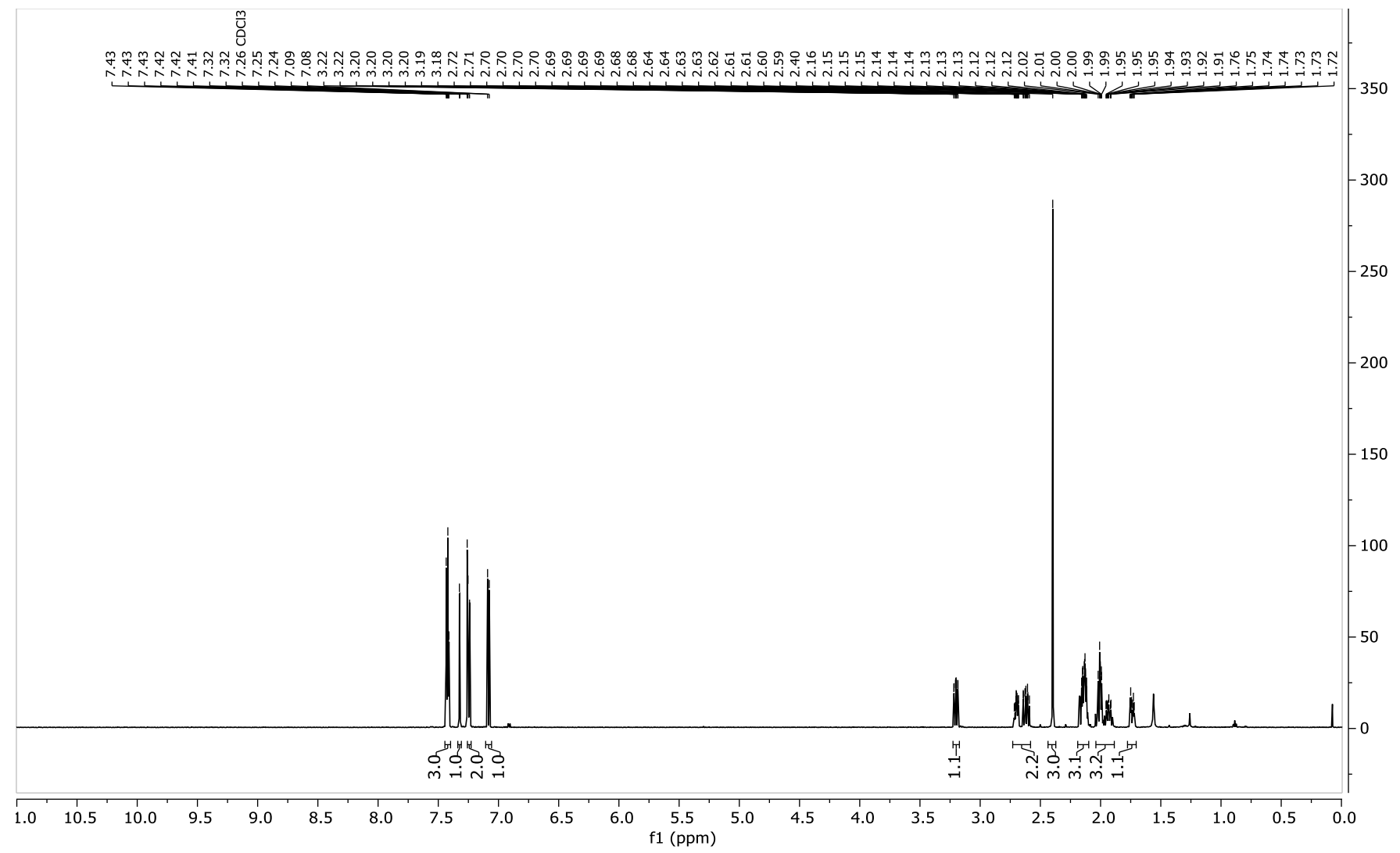

${ }^{13} \mathrm{C}$ NMR (151 MHz, $\left.\mathrm{CDCl}_{3}\right)$

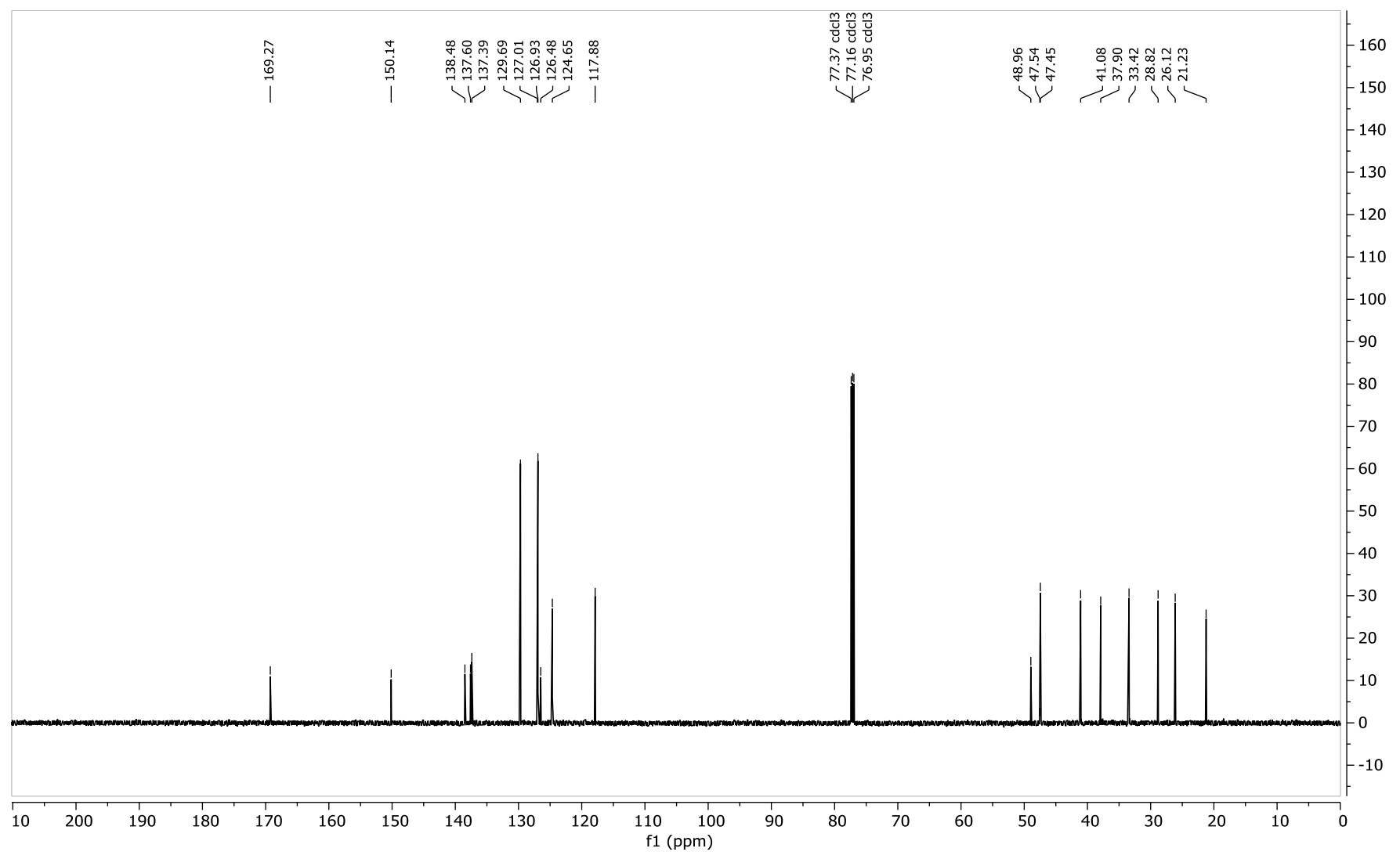


${ }^{1} \mathrm{H}$ NMR $\left(500 \mathrm{MHz}, \mathrm{CDCl}_{3}\right)$

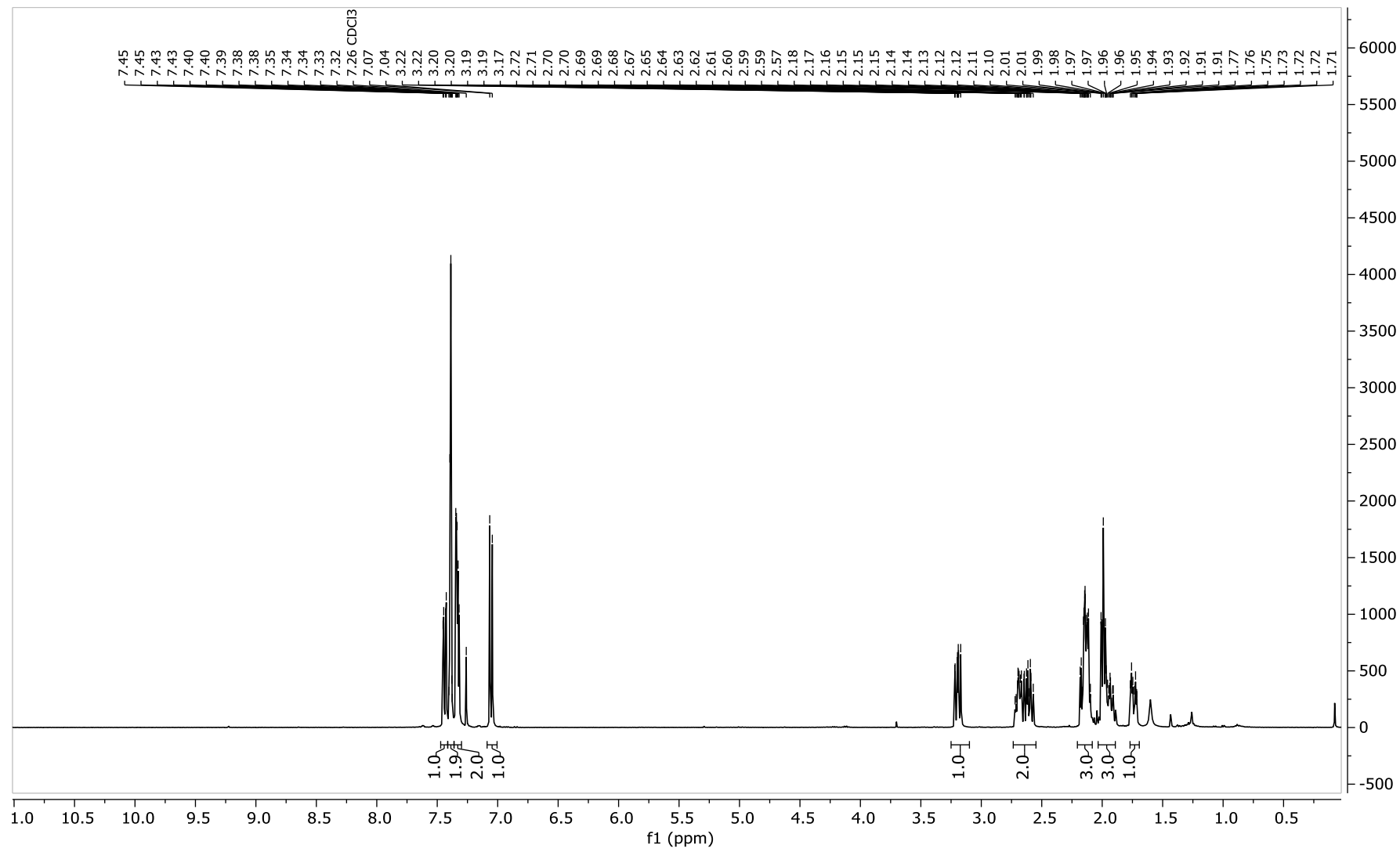

${ }^{13} \mathrm{C}$ NMR (126 MHz, $\mathrm{CDCl}_{3}$ )

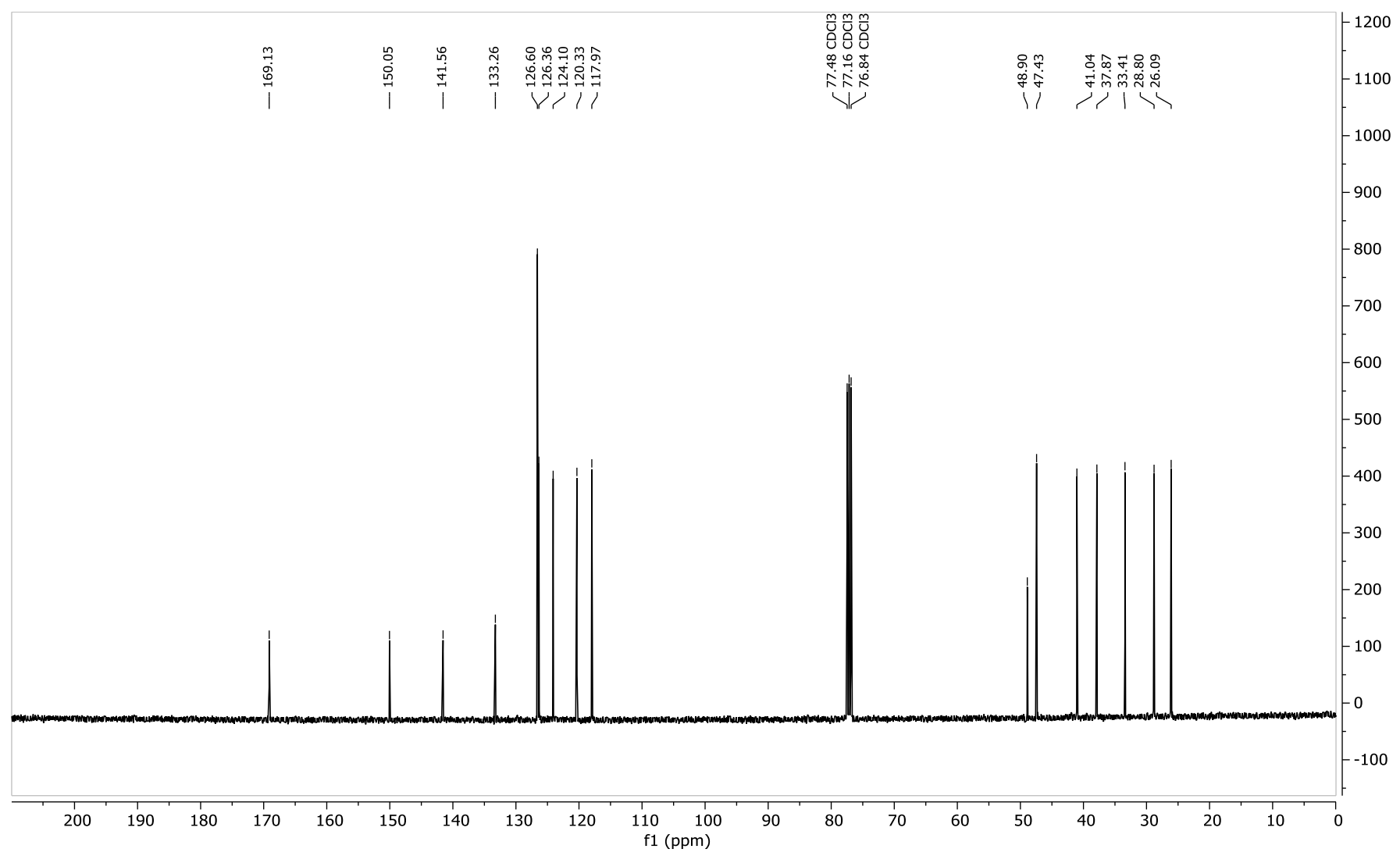


${ }^{1} \mathrm{H}$ NMR $\left(500 \mathrm{MHz}, \mathrm{CDCl}_{3}\right)$

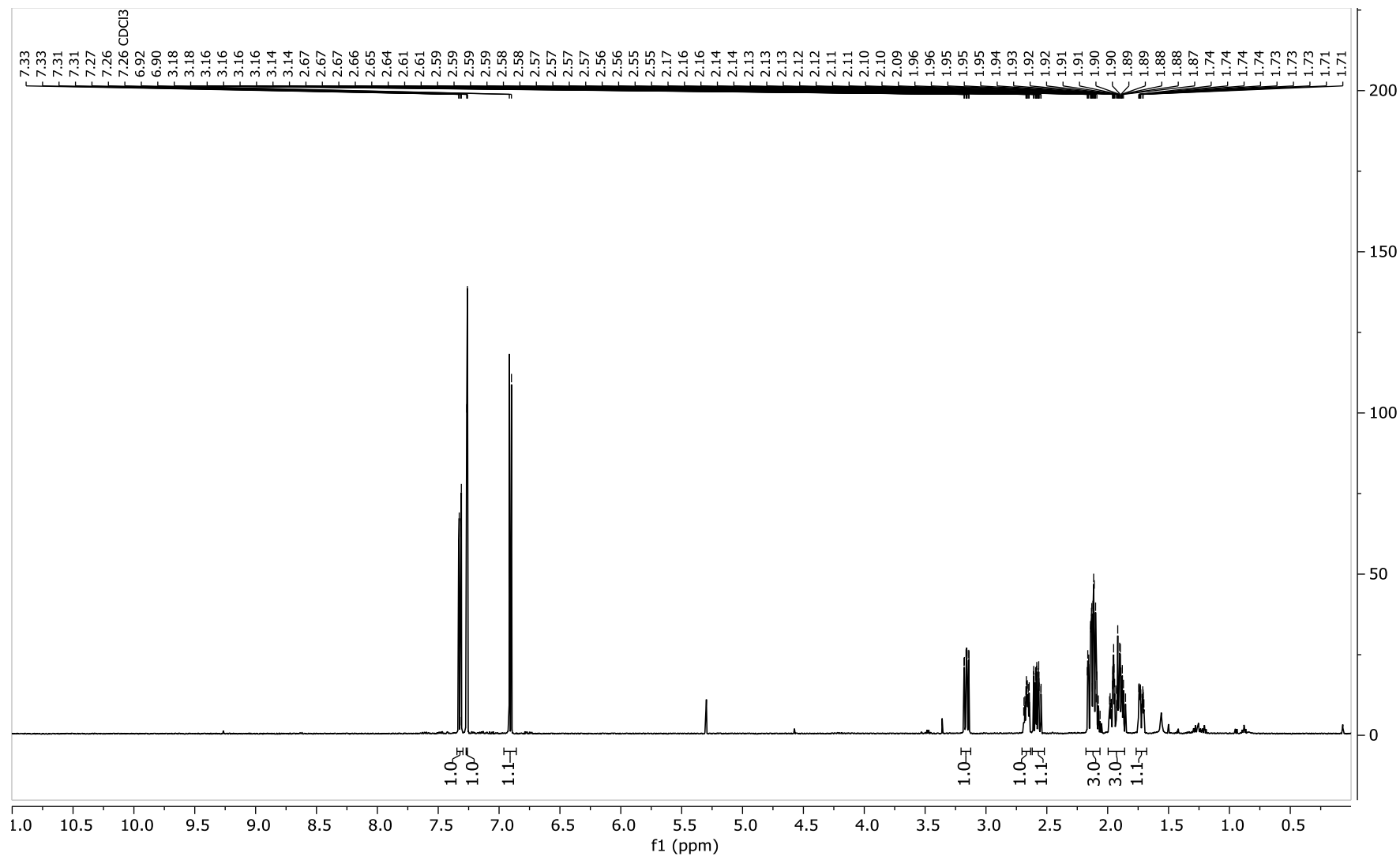

${ }^{13} \mathrm{C}$ NMR (126 MHz, $\mathrm{CDCl}_{3}$ )

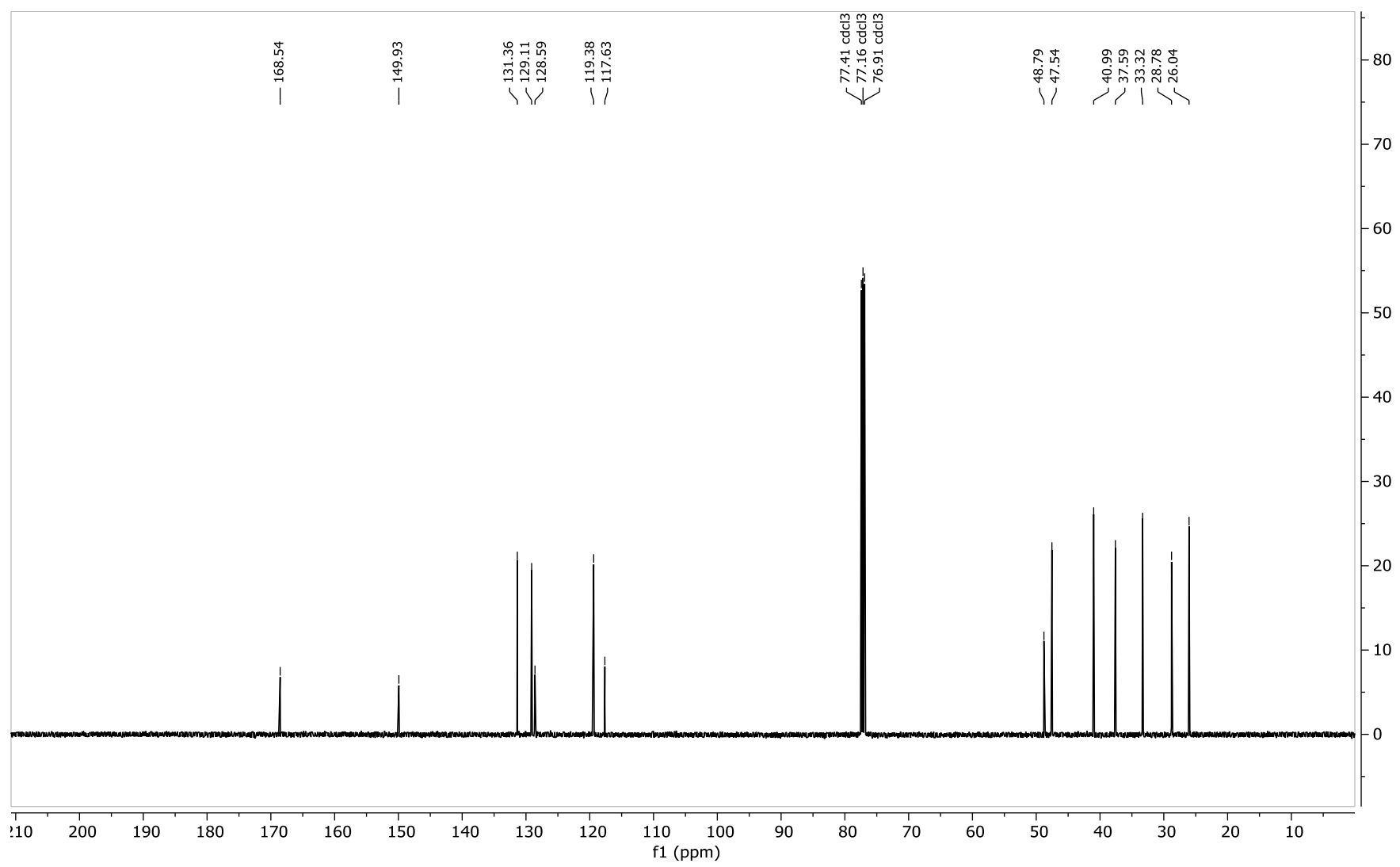


${ }^{1} \mathrm{H}$ NMR $\left(599 \mathrm{MHz}, \mathrm{CDCl}_{3}\right)$

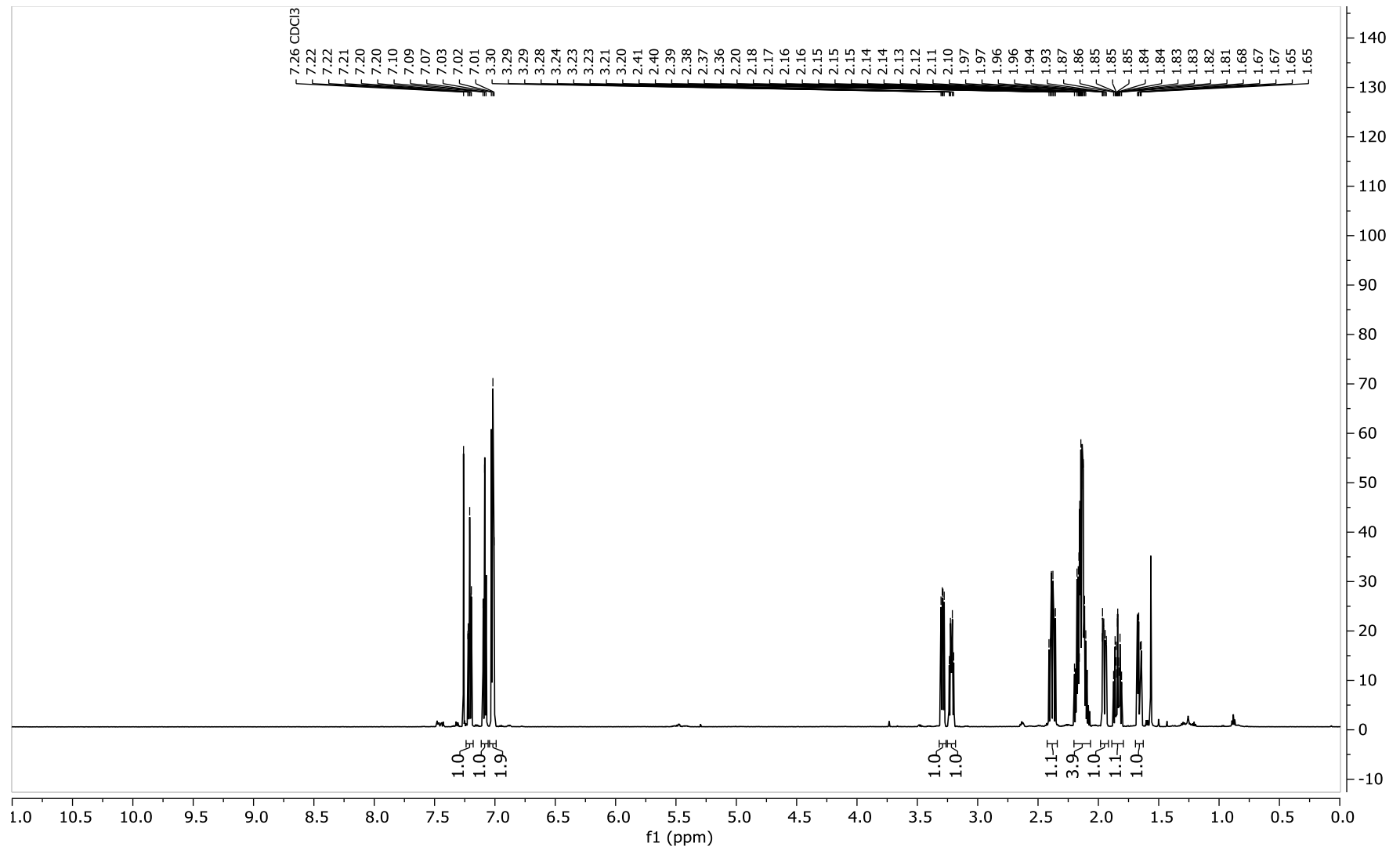

${ }^{13} \mathrm{C}$ NMR (151 MHz, $\left.\mathrm{CDCl}_{3}\right)$

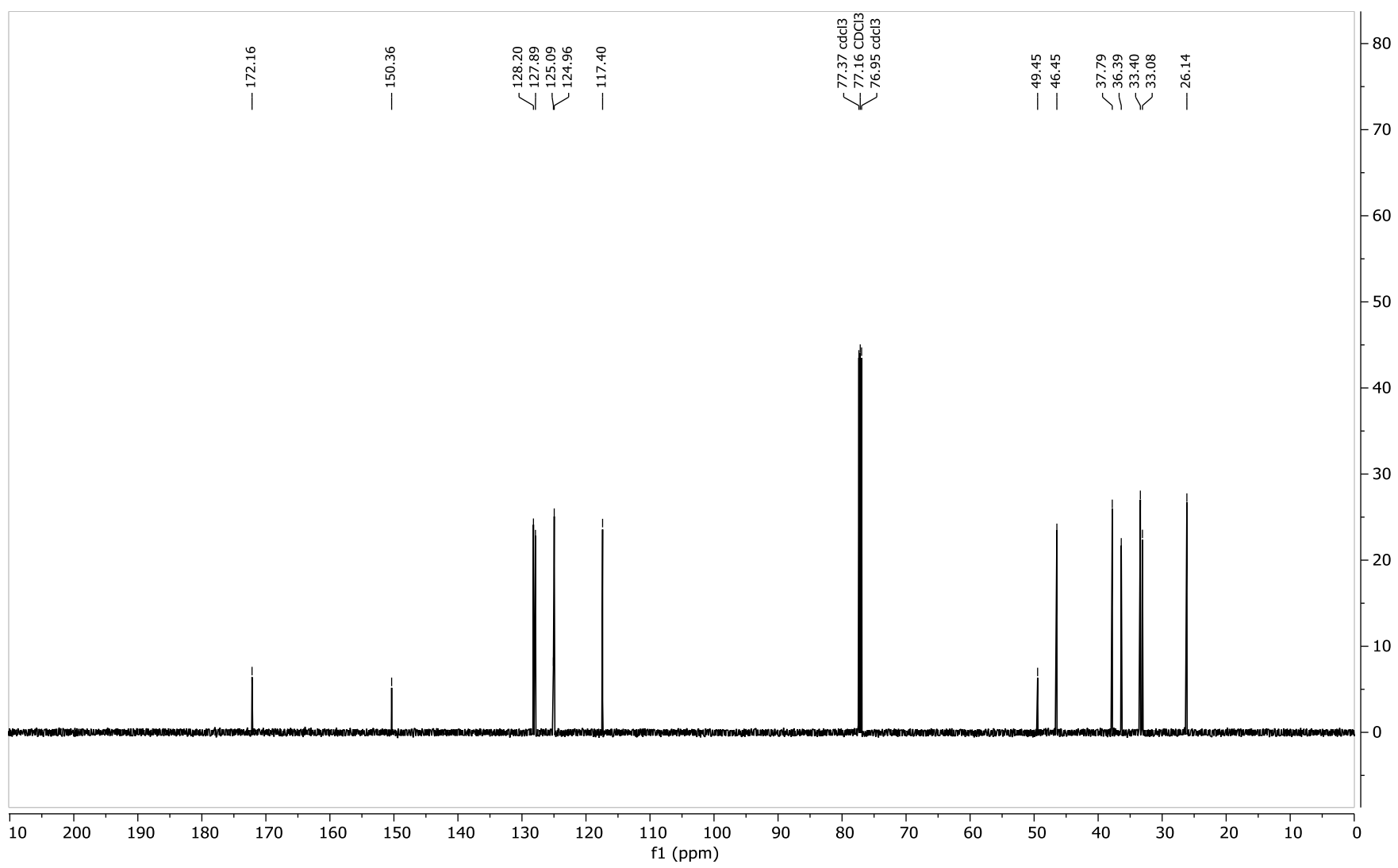


${ }^{1} \mathrm{H}$ NMR (500 MHz, $\mathrm{CDCl}_{3}$ )

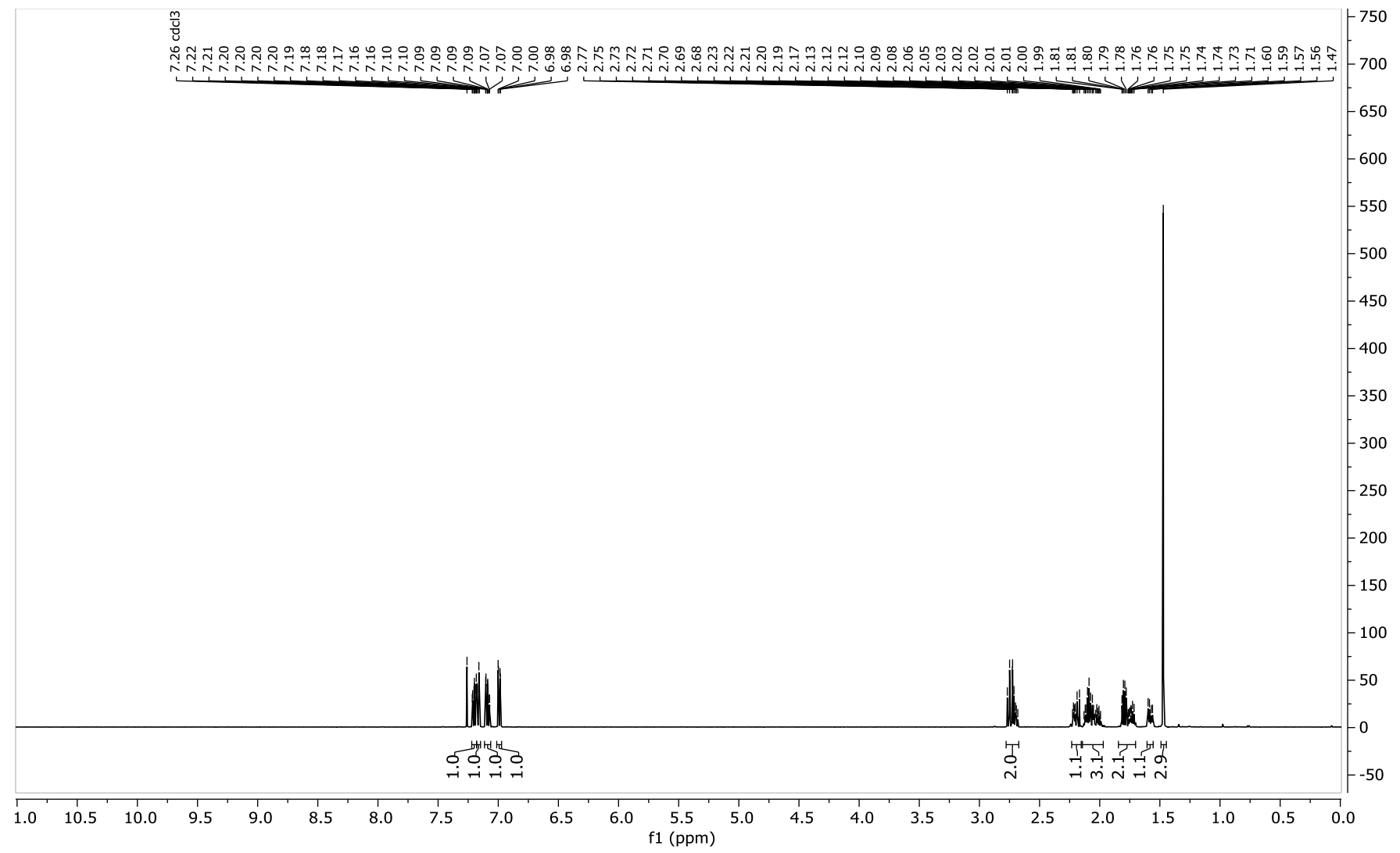

${ }^{13} \mathrm{C}$ NMR (126 MHz, $\left.\mathrm{CDCl}_{3}\right)$

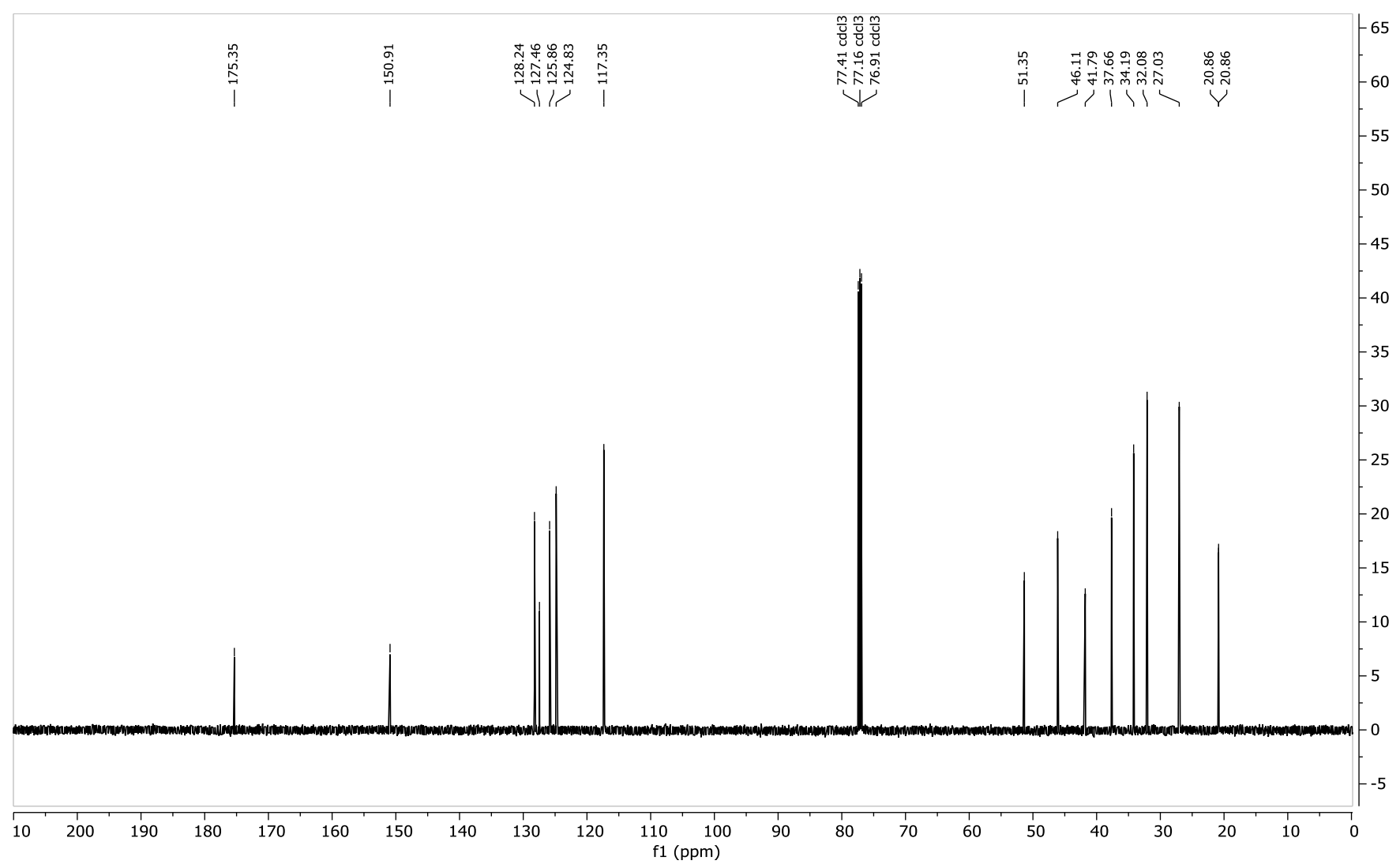

